Wayne State University

DigitalCommons@WayneState

Wayne State University Dissertations

$1-1-2012$

\title{
The mental model comparison of expert and novice performance improvement practitioners
}

Sacip Toker

Wayne State University,

Follow this and additional works at: http://digitalcommons.wayne.edu/oa_dissertations

\section{Recommended Citation}

Toker, Sacip, "The mental model comparison of expert and novice performance improvement practitioners" (2012). Wayne State University Dissertations. Paper 480. 


\title{
THE MENTAL MODEL COMPARISON OF EXPERT AND NOVICE PERFORMANCE IMPROVEMENT PRACTITIONERS
}

\author{
by \\ SACIP TOKER \\ DISSERTATION \\ Submitted to the Graduate School \\ of Wayne State University, \\ Detroit, Michigan \\ in partial fulfillment of the requirements \\ for the degree of \\ DOCTOR OF PHILOSOPHY
}

2012

MAJOR: INSTRUCTIONAL TECHNOLOGY

Approved by:

Advisor

Date 


\section{DEDICATION}

Necip TOKER (1908 - 2001),

who gave his infinite care and love as a grandfather. 


\section{ACKNOWLEDGEMENTS}

To my beloved parents, Nazire and Oktay Toker who have been with me in every milestone of my career with their "quiet" encouragement, countless pride, and eternal belief about my skills.

I deeply acknowledge the love, support, and patience of my adorable fiancé, Gulcan Gul Balcik who has been waiting me to complete one of the most significant works of my academic career. I am greatly fortunate that I have such a heartening partner to spend the rest of my life.

I also express my special gratitude to The Scientific and Technological Research Council of Turkey for their financial support to start my doctoral degree. I will work hard for my country to make this support more invaluable.

Finally, I am cordially appreciative to the members of my committee for their patient support, encouragement, and guidance: my advisor Dr. James L. Moseley, Dr. Timothy W. Spannaus, Dr. Ingrid L. Guerra-Lopez, and Dr. Celia Livermore. 


\section{TABLE OF CONTENTS}

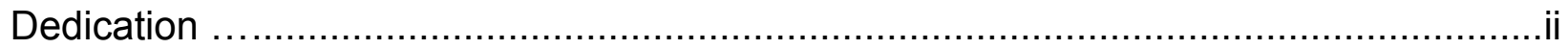

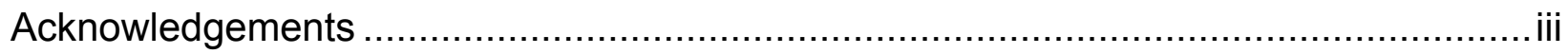

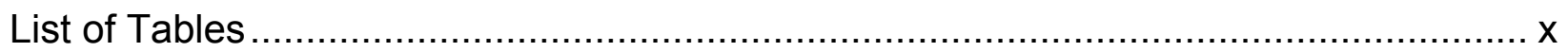

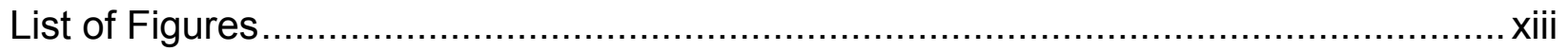

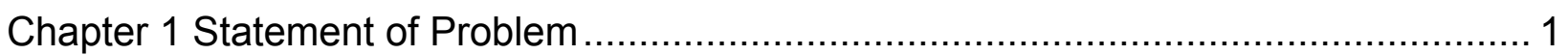

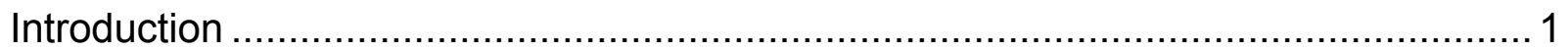

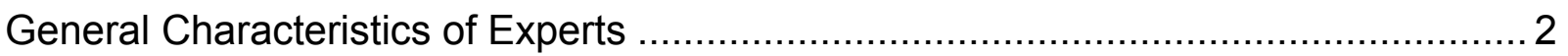

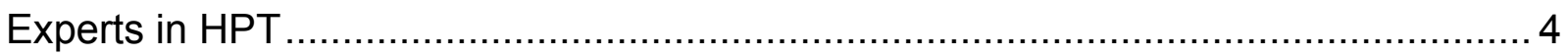

The Nature of Studying Expert versus Novice Comparison ................................... 6

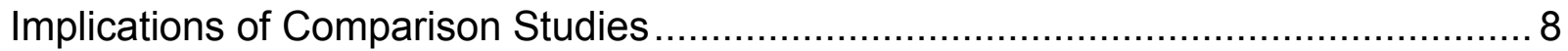

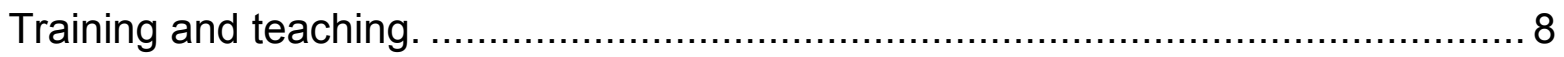

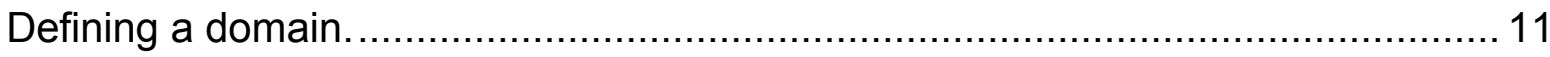

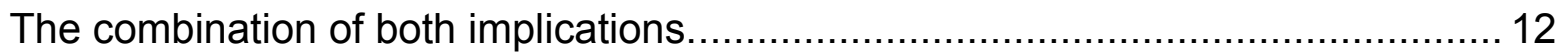

Mental Models: A Means to Reveal Expert Knowledge …................................... 13

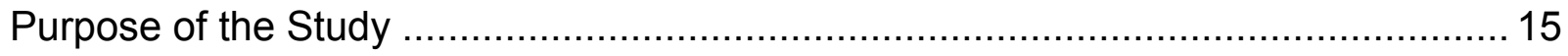

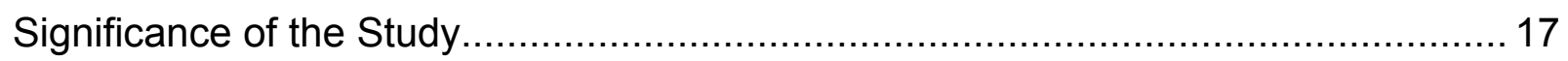

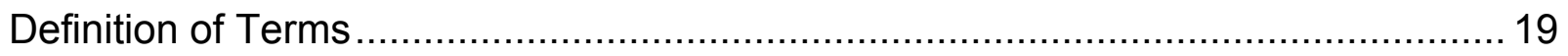

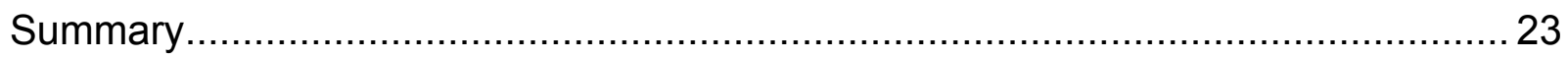

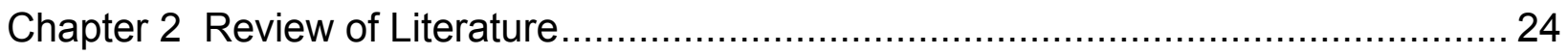


Introduction

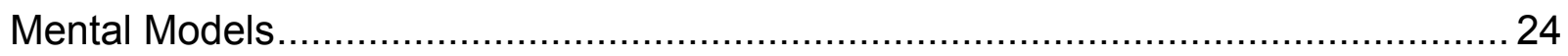

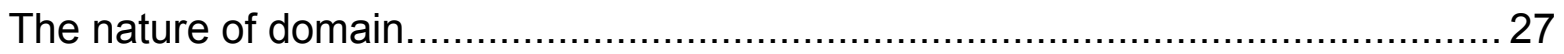

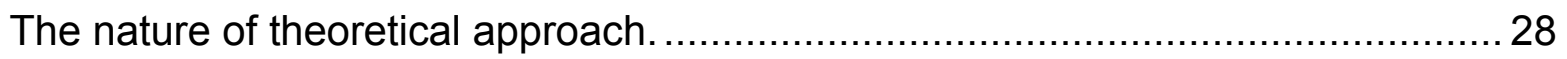

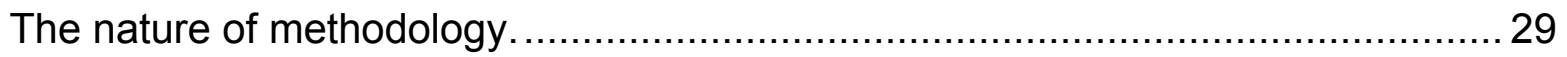

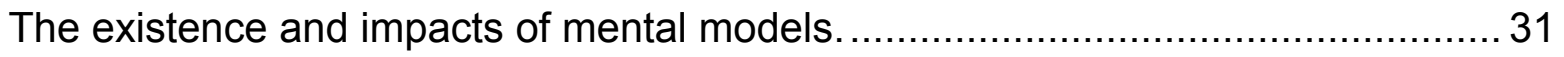

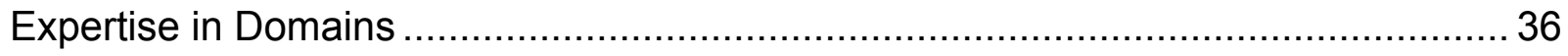

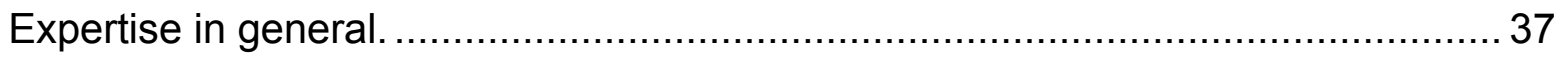

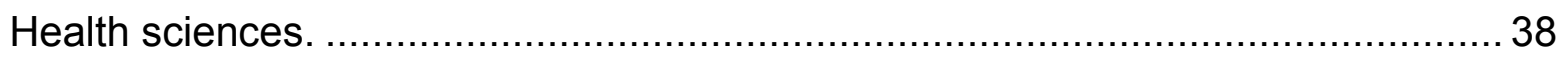

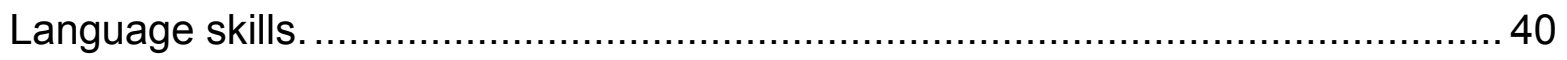

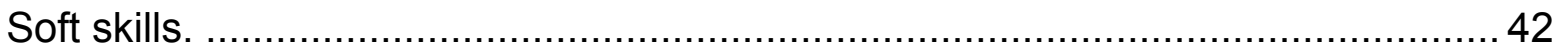

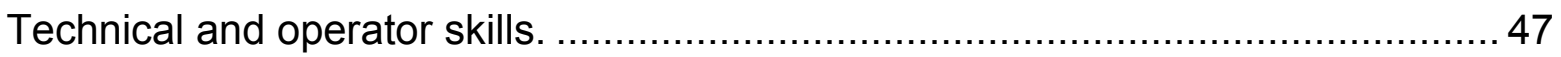

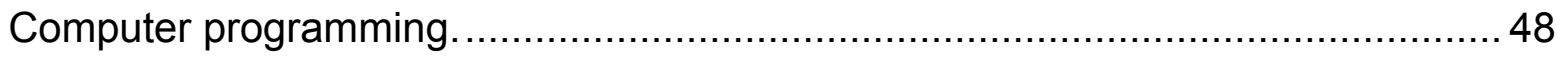

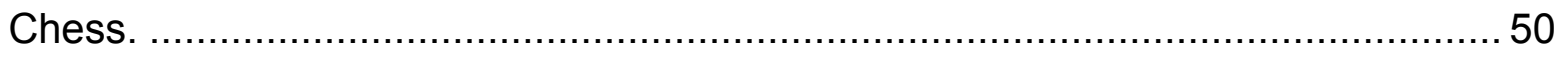

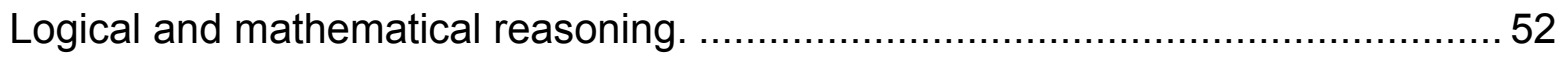

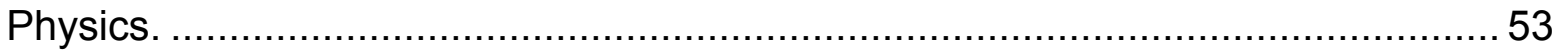

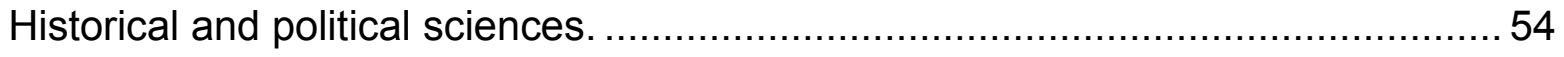

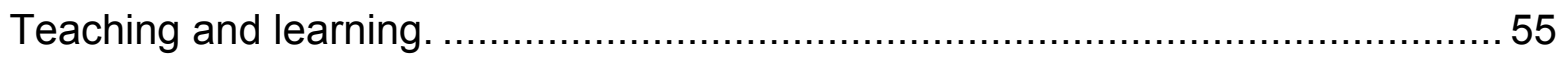

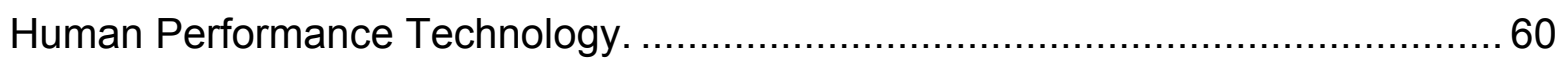

The Relationship of Expertise with Individual Characteristics ................................ 63 
Intelligence.

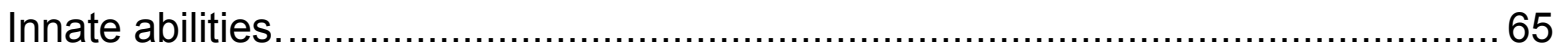

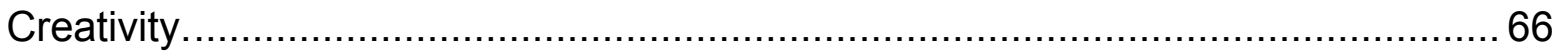

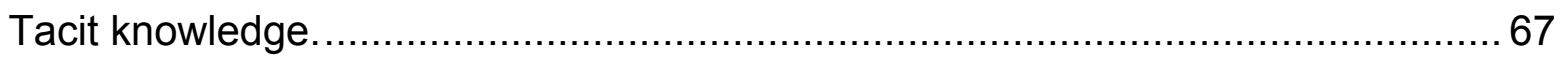

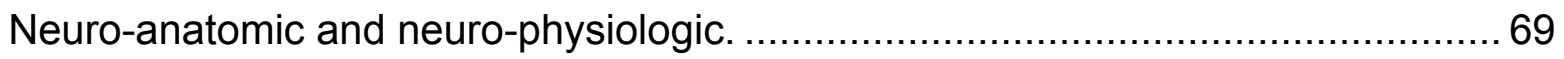

Age

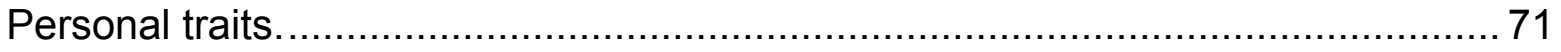

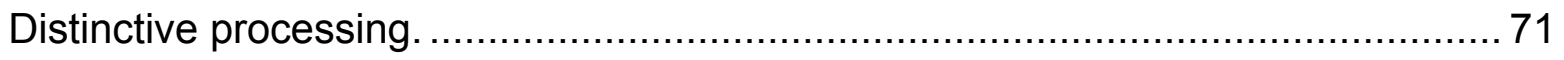

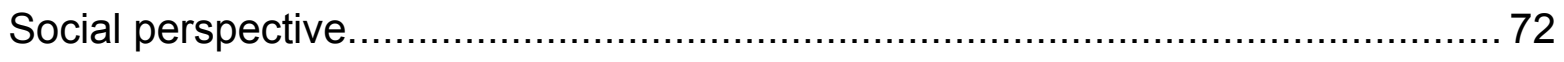

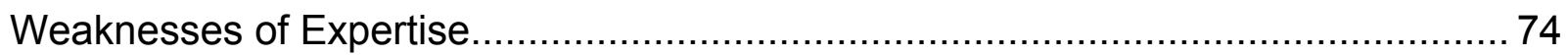

Pathfinder Scaling: An Innovative Technique to Elicit Knowledge Structure ............. 77

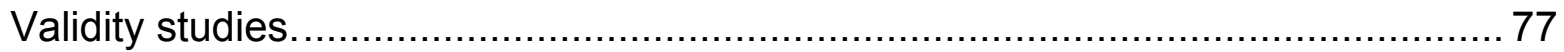

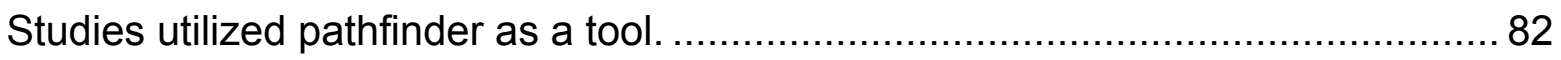

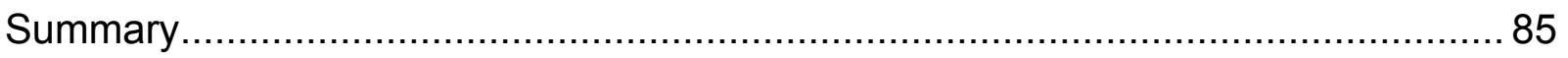

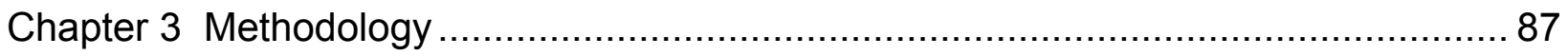

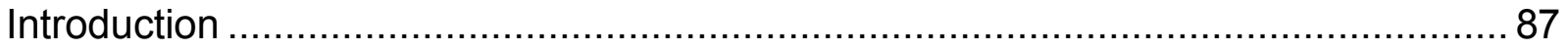

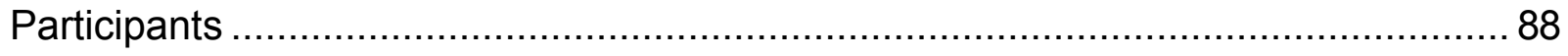

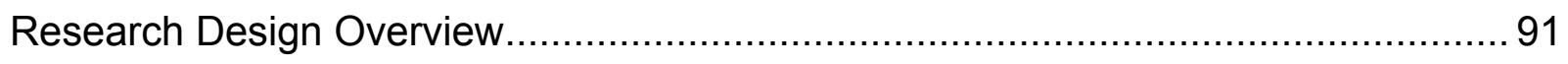

Phase 1: Identification of concepts and expert referent model. .......................... 91

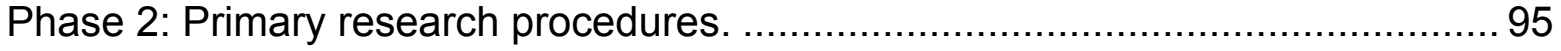


Instrumentation

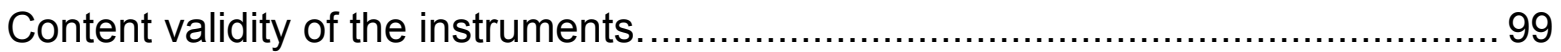

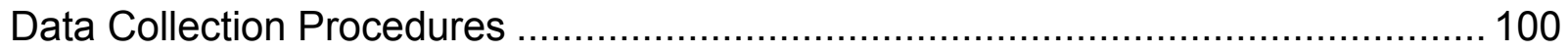

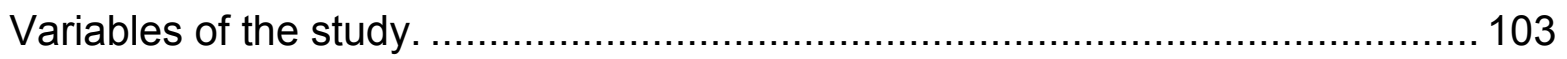

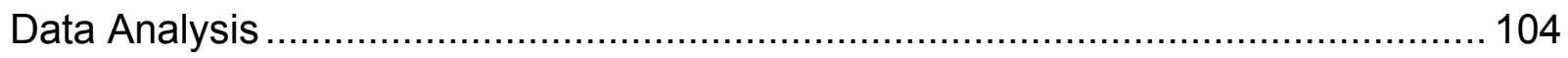

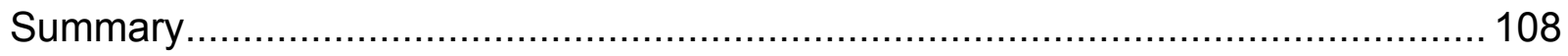

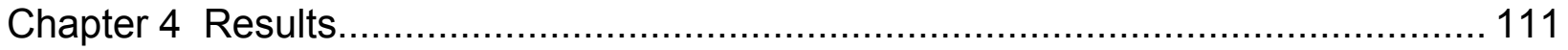

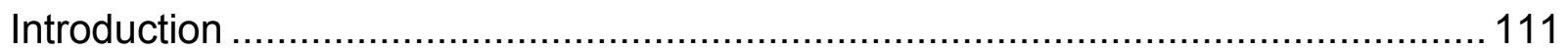

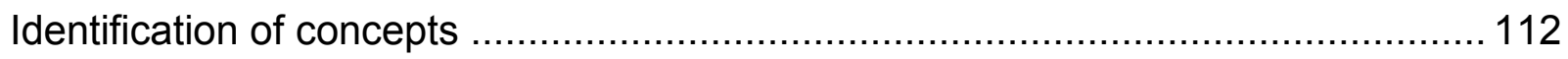

Identification of Expert Referent Model ........................................................... 116

Research Question 1: What Is The Common Mental Model Of Expert Performance Improvement Practitioners? .......................... 116

Research Question 2: What Is The Common Mental Model Of Novice Performance Improvement Practitioners?

Research Question 3: What, If Any, Are The Similarities And Differences Between The Common Mental Models of Experts And Novices?

Research Question 4: What Are The Professional Profile Characteristics Of Performance Improvement Practitioners And Experts? ........ 123

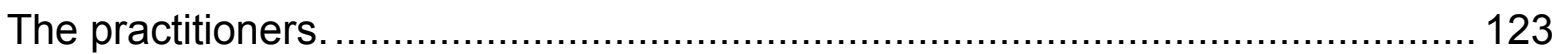

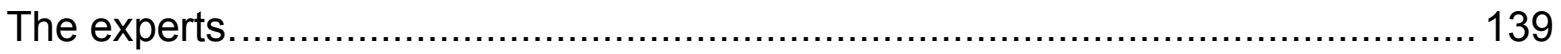

Research Question 5: To What Extent Are The Professional Profile Characteristics Of Performance Improvement Practitioners Associated With Their Mental Models Of Expertise Derived From Pathfinder? 153 
Summary.

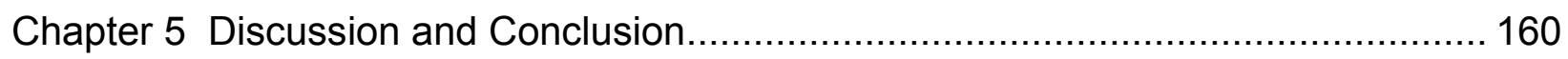

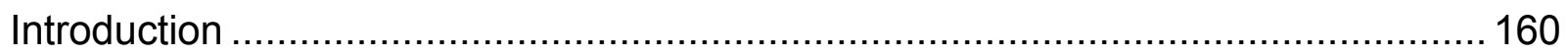

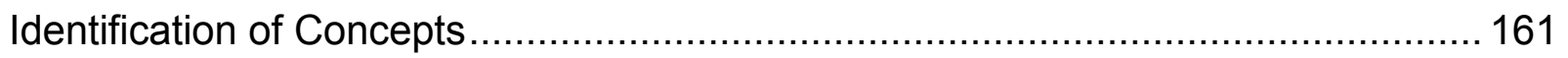

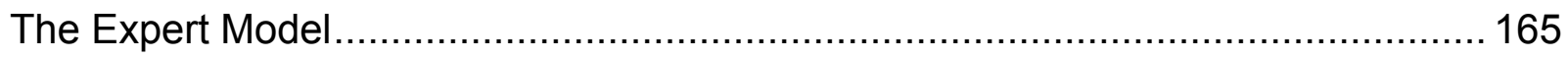

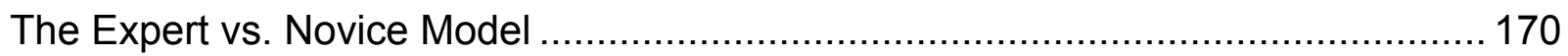

Relationship between Professional Activities and Mental Model of Expertise.......... 172

Demographic Characteristics and Mental Model of Expertise .............................. 179

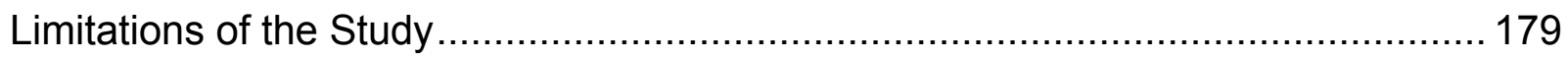

Implications for the Field of Performance Technology ...................................... 182

Recommendations for Further Research ....................................................... 185

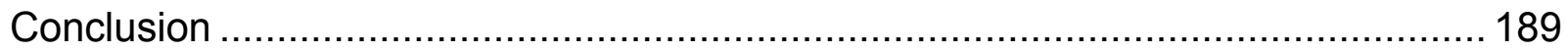

Appendix A: Wayne State University Human Investigation Committee Approval

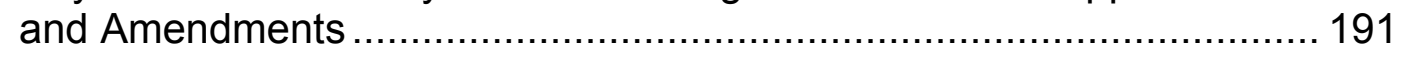

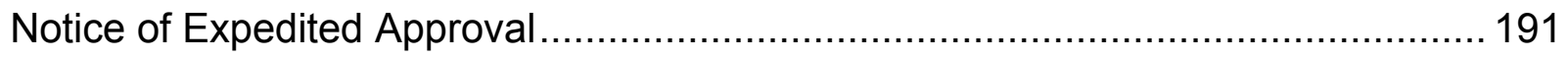

Notice of Expedited Amendment Approval - June 02, 2011 ............................... 192

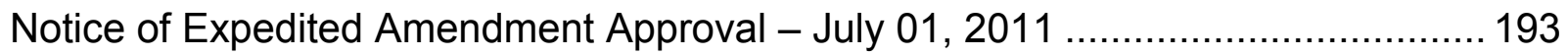

Appendix B: Information Sheets for Participation to Research ............................... 194

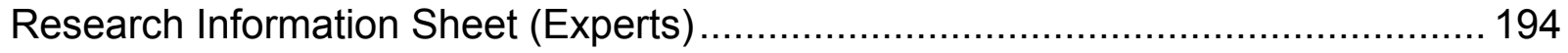

Research Information Sheet (Participants) …….............................................. 196 
Appendix C: E-mail Cover Letters for Invitation to Participate.

Appendix D: The Support Letters from the Professional Organizations

202

International Society for Performance Improvement (ISPI) ................................. 202

The American Society for Training and Development (ASTD) .............................. 203

Association for Education Communications and Technology (AECT) .................... 204

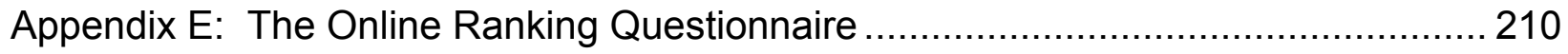

Appendix F: The Concept-Pairs Comparison Questionnaire .................................. 212

Appendix G: The Professional Profile Characteristics Questionnaire ........................ 214

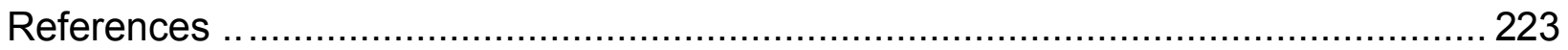

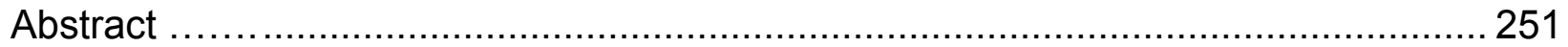

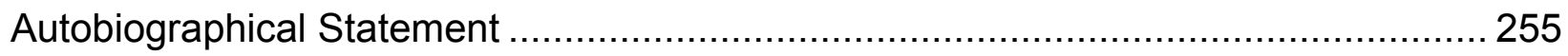




\section{LIST OF TABLES}

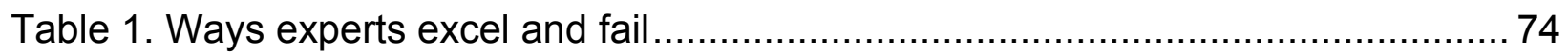

Table 2. 30 concepts selected for the Online Ranking questionnaire ......................... 92

Table 3. A summary of research questions ….................................................. 107

Table 4. Ranking, total, and weighted total scores for 30 concepts ........................ 113

Table 5. How long have you actively been in the HPT field? ................................. 124

Table 6. The practitioners' professional activities corresponding to deliberate

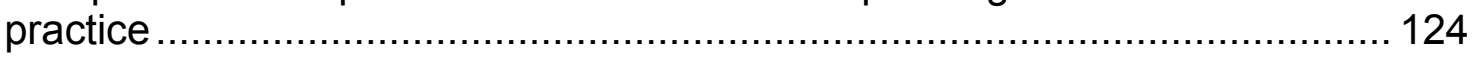

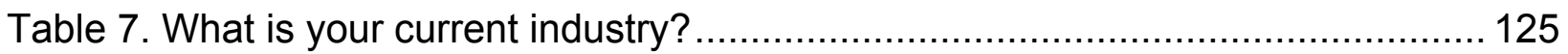

Table 8. Which of the following industries have you have worked or completed

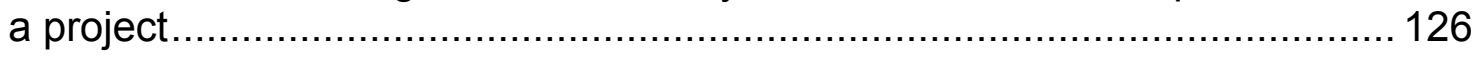

Table 9. How many different organizations have you worked so far? ....................... 127

Table 10. What kinds of HPT related project(s) have you worked? ......................... 128

Table 11. Which of the following degree(s) do you have or are you pursuing? .......... 129

Table 12. Which of the following certificates do you have? ..................................... 130

Table 13. The Number of Publications by Types................................................. 131

Table 14. How many professional presentations (i.e., how-to, educational/lecture, case study, business, futurecasting, issues, etc.) or workshops have

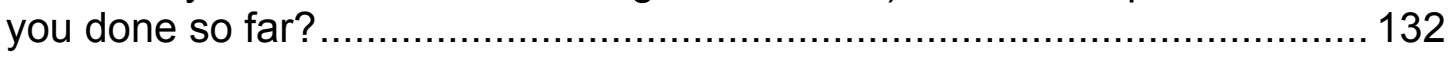

Table 15. The number of courses that the practitioners taught by course formats ...... 132

Table 16. Membership to professional organizations ............................................ 133

Table 17. The number of the participations to the conferences............................... 134

Table 18. Board membership in professional organizations ..................................... 135

Table 19. Volunteer activities in the professional organizations ............................... 136

Table 20. The grouped job titles of the participants............................................. 137 
Table 21. The practitioners' ages

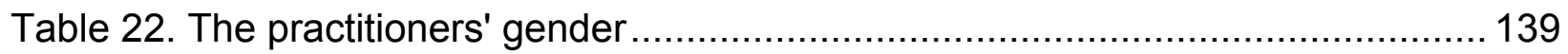

Table 23. How long have you actively been in the HPT field? ................................ 140

Table 24. The experts' professional activities corresponding to deliberate practice $\ldots . .140$

Table 25. The experts' current industries

Table 26. The experts' industries in which they have worked or completed a project.

Table 27. The number of organizations which experts worked .............................. 142

Table 28. The types of projects that the experts worked ....................................... 143

Table 29. The degree completed or currently being pursued by the experts.............. 144

Table 30. The certification ownership of the experts .................................................. 145

Table 31. The Number of Publications by Types.................................................... 146

Table 32. The professional presentations (i.e., how-to, educational/lecture, case study, business, futurecasting, issues, etc.) or workshops done so far by the experts

Table 33. The number of courses that the experts taught by course formats............. 147

Table 34. The experts' membership to professional organizations …....................... 148

Table 35. The number of the participations to the conferences of the experts ............ 149

Table 36. The experts' board membership in professional organizations.................... 150

Table 37. The experts' volunteer activities in the professional organizations .............. 151

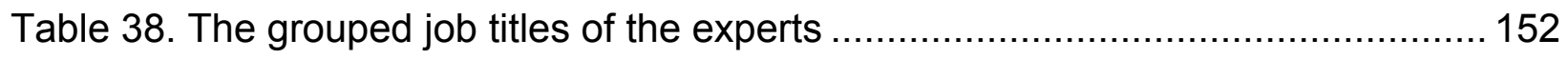

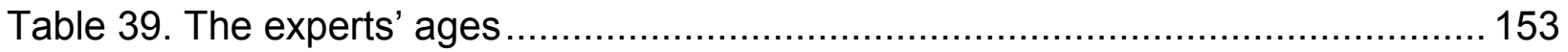

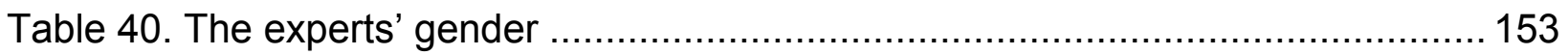

Table 41. The correlations between Mental Model of Expertise and Six Professional Profile Characteristics 
Table 42. The stepwise regression analysis coefficients results for the mental model of expertise and five professional profile characteristics .................. 157 


\section{LIST OF FIGURES}

Figure 1. The expert referent model generated from 16 experts' proximity data ......... 117

Figure 2. The common mental model of novices from 33 novices' proximity data....... 119

Figure 3. Comparison of the common mental models of experts and novices ........... 121 


\section{CHAPTER 1}

\section{STATEMENT OF PROBLEM}

\section{Introduction}

Human Performance Technology (HPT) is an emerging field requiring empirical studies to survive. Scholars in the field constantly emphasize the criticality of research, e.g., Brethower (2000), Klein (2002), Stolovitch (2000), Sugrue and Stolovitch (2000), Foshay, Moller, Schwen, Kalman, and Haney (1999), Foshay and Moller (1992), and Swanson (1988). This constant emphasis is expected to result in more empirical research efforts in the field. In addition to that, a more recent study, which utilized a 15expert panel, by Pershing, et al. (2008b) concluded that the field currently needs to create an integrative framework for the existing research. Marker, Huglin and Johnsen (2006) suggested that not only conducting more empirical research but also defining and prioritization of the most important research questions would be beneficial.

In the light of their previous effort, Huglin, Johnsen, and Marker (2007) conducted a Delphi study to identify the top research priorities in HPT. The priorities were revealed as experts' opinions that are more distinguishable than the aforementioned general suggestions since these may better reflect actual research trends in the field. Their main purpose was to provide a direction for scholars as well as research studies in the field. They discussed five broad categories for tentative research areas: (a) operational definitions of key research variables, (b) measurement of added-value, (c) identification of best practices for optimizing interventions, (d) comparison of the added-values of HPT and other fields, and (e) divulge of differences between experts and novices in terms of their mental models and practices. Each area contained several specific 
research questions. Since these questions were revealed from experts in the field, they represent critical directions for scholars. The present study was derived from the difference between experts and novices in their use of mental models and practices, and it is designed to be a step to fulfill one of the top research priorities.

This section first focuses on general expertise and then considers HPT expertise to provide an understanding of expertise and characteristics of experts. Next, it will elucidate expert research approaches commonly applied and put an emphasis on the approach of expert versus novice comparison study, which will be the core research design of the proposed study. Moreover, the implications of the comparison studies will highlight the potential significance of the results that the proposed research will provide. Finally, the conclusion will provide strengths and weaknesses in the field, research questions, and future work. Chapter 1 is organized into the following five sections: (a) general characteristics of experts, (b) experts in HPT, (c) the nature of expert versus novice, (d) implications of comparison studies for training and teaching and defining a domain, (e) mental models, (f) purpose and significance of the study, (g) definitions, and (e) summary.

\section{General Characteristics of Experts}

Experts, in general, are individuals who possess a large body of knowledge and procedural skills (Chi, Glaser, \& Rees, 1982). They not only possess knowledge and skills but also perform at least two standard deviations above the mean level in the population (Ericsson \& Charness, 1994). In other words, they demonstrate exceptional performance compared to other regular individuals who have been presented as performing at either the intermediate or novice level. 
The terms expert as well as expertise have created a new dimension, which is a comparison between high and less intelligent or skilled individuals, for four decades. Usually, this comparison has been observed as experts versus novices in either practical or research areas. Hence, experts and novices empirically differ with respect to their information processing quantity and organization skills in diverse domains, such as computer programming (McKeithen, Reitman, Rueter, \& Hirtle, 1981), community health occupational therapy (Mitchell \& Unsworth, 2005), nursing (Ericsson, Whyte, \& Ward, 2007), the diagnosis of clinical cases (Boshuizen \& Schmidt, 1992). Moreover, experts have the advantage of accessing a complex network without any conscious retrieval process for remembering or usage.

Chi, et al.'s (1982) seven consecutive and extensive empirical studies brought a different perspective on the difference between expert and novice performances and demonstrated that expert knowledge structures contain fundamental principles and procedural knowledge and associations; whereas, novice structures have superficial features and declarative knowledge. However, Posner (1988) discussed that expert knowledge is more associated with elaborate semantic memory, which is a portion of declarative knowledge and related to meaning, ideas, and concepts independent from personal experiences rather than a general reasoning process. He further stated that such memory is not only specific to unusual people but also very broad to everybody; therefore, although they do not have gifted talents, everybody can be an expert in a domain. Although Posner's (1988) differed from that of Chi, et al.'s (1982) in that it constrained expert knowledge to declarative knowledge, especially crucial was Posner's (1988) disassociation of expert knowledge from the innate talents of individuals. The 
clear evidence confirming the aforementioned point stemmed from systematic laboratory research demonstrating that the exceptional abilities of geniuses and savants did not present any evidence for the impact of giftedness or innate talent; nevertheless, these abilities were mostly acquired when optimal environmental conditions were present (Ericsson \& Charness, 1994). These conditions were coaching, one-to-one training, or long-term training programs.

Since the present research study is related to experts in the HPT field, the next section will provide several studies that cover some characteristics of HPT experts. Then, summary of general characteristics and HPT experts' studies will be presented.

\section{Experts in HPT}

Villachica, et al. (2001) studied the cognitive map of HPT experts, the relationship between this cognitive map and HPT expertise characteristics, e.g., the number of published books or book chapters, years of HPT practitioner experience, the numbers of juried or non-juried articles or presentations, and the similarities and differences between the cognitive maps of experts and novices. They found that only the number of published books was significantly associated with the expert cognitive map. The authors also explained that the key concept was identified as results in expert cognitive maps. Instructional systems design, human performance technology, business cases, workplace organization, and human capital concepts were the main groups branching from the results concept. Each expert's cognitive map was similar within the expert group but significantly different from those in the novice group.

Witucki (2006) investigated the relationship between the expertise of performance technologists and the way in which they acquire knowledge and apply it to 
specific performance interventions. He found that both the level of expertise and the way that expertise was acquired had less influence on the usage of interventions. The study also revealed that novices acquired their intervention knowledge via formal education; whereas, the experts acquired theirs by informal education.

Since HPT is a relatively new field, scholars must borrow numerous concepts and theories from other cognate fields (Johnsen, Huglin, \& Marker, 2008), such as human resource management, organizational development, training and development, instructional design, among others. Many HPT experts believe that HPT is an alternative field of study that covers also training and development and instructional design (ID) fields (Januzewski \& Molenda, 2007). For this reason, experts from these fields are also accepted as experts in HPT.

Perez and Emery (1995) studied the differences in thinking between expert and novice instructional designers (IDs) on the same given task. They found that novice and expert IDs employed different courses for their design tasks. Expert IDs spent more time qualitatively analyzing and interpreting every detail of a design problem to reveal the big picture; in contrast, novices devoted their time to understanding the design problem. Furthermore, expert IDs thought about all possible solutions and relations at the same time; whereas, novices focused on fewer considerations and one at a time.

In summary, experts obviously perform better than novices in their domains. They have prompt information processing abilities, easy transformation of fundamental principles into applicable information skills, complex knowledge of organizations, efficiently grouped information for different and unusual cases, and hierarchically associated knowledge structures. According to Winn (2004), automaticity and 
knowledge organization are two cognitive processes that account for attaining expertise. The first two characteristics above explain automaticity; the next three characteristics explain knowledge structures, that is, mental models. HPT experts also have domainspecific characteristics in addition to the aforementioned general characteristics. HPT experts are the individuals who are informally educated about the field; their expertise level can be identified by the number of the books they have published. They mostly focus on the results and structure of all the other concepts around them.

Experts need to be supported by certain conditions, such as coaching; however, individual giftedness is not a significant factor for expertise. Since experts are a source of extensive knowledge and experience, there is an enormous amount of attention paid to their characteristics. Experts have been a core part of numerous scientific studies as either participants or information providers for decades in diverse types of research designs. The next section will cover common research types focused on experts and expertise, and it will specifically explain the study of expert and novice comparison.

\section{The Nature of Studying Expert versus Novice Comparison}

The approaches utilized for the study of expertise are twofold (Chi, 2006): (a) study of truly exceptional people to realize the underlying mechanism of their performance and (b) comparison of experts to novices. She also stated that, currently, the terms of expertise and expert cannot be identified precisely (Chi, 2006). Nevertheless, they are commonly explained relative to novices on a continuum. This has created a reasonable rationale for comparison studies. Chi (2006) also emphasized that the comparison of expert to novice research approach is very critical in terms of not only explaining how experts excel but also helping people learn and become an expert. 
Schoenfeld and Herrmann (1982) confirm that the comparison of novices and experts for understanding the exceptional performance and expert perception as a starting point for people who are not able to perform like an expert does not explain the relationship between perception and expertise completely. It only provides an interpretation and investigation of the relationship between expert and novice in a continual process. They especially accept the nature of the expert and novice continuum and its importance. A detailed analysis of this continual process helps the comparison research approach understand how we can facilitate less skilled or experienced people to become more skilled; moreover, the necessary conditions might be identified during the process of becoming an expert, such as, deliberate practice (Ericsson, 2008; Ericsson \& Charness, 1994; Ericsson, Krampe, \& Tesch-Römer, 1993; Ericsson, Prietula, \& Cokely, 2007) and the level of exposure to the expertise domain (Hoffman, 1998).

In summary, the study of expert versus novice comparison is important to identify the process of becoming an expert because it reveals the dynamics occurring during the process. However, focusing on immediate occasions might not be as beneficial as investigating the whole process. Expertise is a phenomenon that includes behaviors and accomplishments. For expertise, behaviors are the basis for the required knowledge, skills, and abilities; accomplishments provide the basis for criteria. Only analyzing accomplishments show the results of expert performance; however, it might not provide information about the behaviors that mediate the accomplishments. Since the comparison study considers both behaviors and accomplishments, it is an effective method to understand expertise. For this reason, there have been numerous examples 
of these studies in diverse fields, and they constantly emphasize the consequences and implications. Several areas that might take advantage of these implications will be discussed in the next section.

\section{Implications of Comparison Studies}

The most common suggested implications of expertise comparison studies are related to training and teaching, defining a domain, and both of training and teaching and defining a domain. This researcher considers these implications since the current literature review as well as the proposed research will provide similar implications for the field; as a result, this supports the significance of the study. The following section will explain these implications under three separate titles.

Training and teaching. The key differences between experts and novices trigger a knowledge-based conception of intelligence that might lead to different strategies for how individuals might be taught or trained (Chi, et al., 1982). For example, Zahodnic (2009) investigated performance of novices who are exposed to a think aloud process of an expert performer while they were using simulations in which they needed to make decisions via gathering information and decreasing errors of omission. $\mathrm{He}$ found that think aloud protocol of an expert as an instructional strategy improves novice's performance regarding choosing the best information as well as decision making. Charness \& Tuffiash (2008) discussed that the current training programs for skills improvement might be structured and designed more effectively by the interpretation of the underlying mechanisms of expert performance, and they found that training programs transferring task-related knowledge and skills that were eminent to expert performance demonstrated skill acquisition among non-experts and the reduction 
of expert errors on representative tasks. A similar suggestion proposed by Perez \& Emery (1995) in their comparison study of novice and expert thinking in instructional design included that expertise identified from the results could be used for training programs that aim to improve individuals' expertise. Villachica, et al. (2001) asserted that the cognitive map of HPT experts, which was generated in their empirical study, could be used to develop competencies and a good mind tool to introduce the field to new practitioners. Their implications cover not only training purposes but also job-aids, on-the-job self-training techniques.

Skill-acquisition of a specific domain, which is one of the primary goals of advanced degree programs, can be better explained in five stage continuum that are novice, advanced beginners, competent, proficient, and expert (Dreyfus \& Dreyfus, 1986). Each stage is differentiated in light of task perceptions and decision making styles. Novices interpret a task situation as context-free objects and apply standard rules or procedures disregarding situational circumstances. One goes to the advanced beginner stage when (s)he starts discriminating context-free and context-dependent objects. At the competent stage, one starts adopting standard rules or procedures to the situation. In the proficiency level, one intuitively understands and organizes tasks, but there is some analytical thinking about what to do. At the expert level, one acts based on established and experienced understanding. One uses his/her own skills without awareness. Experts normally do what they need to do rather than focusing on making decisions and solving problems in a condition. The skill-acquisition theory explains one's path to expertise regardless of domains. The theory enlightened many different domains by providing a framework for preparing domain experts, such as Benner's 
(1984) influential theory on nursing expertise (Gobet \& Chassy, 2008). This is a good example to demonstrate the direct influence of expertise theories on educating or training domain practitioners. At the individual level, Ericsson \& Charness (1994) demonstrated in their extensive empirical study of violinists and pianists that the investigation of expertise and its applications had significant consequences and implications in order to comprehend optimal human adaptation and learning. For example, they observed that the most prominent scientists published their seminal publications ten years after their first publications, and they spent four hours every day specifically on writing. These results are remarkable since they provide highly applicable implications for those who are responsible for training scientists.

Expertise comparison studies also enlighten the discussion of the time period of training for exceptional performance. Schoenfeld \& Herrmann (1982) studied the effect of short time training on novices' perception regarding problem solving. They indicated that short term training might show strong improvement on novices' perception regarding problems in a domain, but they were not sufficient to make their perceptions be truly expert-like. Moreover, a very long period of active learning that mainly focuses on refining and improving skills and performance on a specific domain was essential for exceptional performance that experts show without taking advantage of their inherent talents (Ericsson \& Charness, 1994). On the other hand, training as well as instruction is a necessary but not sufficient condition for expertise since it also requires maturity and experience on the job (Lave \& Wenger, 1991). For instance, Feltovich, Johnson, Moller, and Swanson (1984) proposed several procedural suggestions that stemmed from work settings to change or update current medical training programs to ensure the transition 
of knowledge creation and problem-solving from noviceness and expertise after concluding their empirical study. Moreover, Dew, Read, Sarasvathy, and Wiltbank (2009) criticized a discrepancy that was the indirect effect of entrepreneurship research and accumulated invaluable knowledge and information as a result of it on course development in Master of Business Administration (MBA) programs based on the empirical study that they recently conducted. They compared the logic frame of experts and novices during entrepreneur decision making in order to close this discrepancy. Their study showed that expertise research would be a good source for real life information to design and develop current curriculum and courses in advanced degree programs.

Defining a domain. When there are efforts to define a new domain, the network of one or more experts would be an outstanding foundation to indicate the basic organization of the domain knowledge and the structural enhancements derived from superior performance (Bradley, Paul, \& Seeman, 2006). For instance, Villachica, et al. (2001) for HPT and Perez and Emery (1995) for ID suggested new frameworks for the conceptualization of the fields based on their results. Since these authors applied comparison studies, they had an opportunity to distinguish factors that provided the basis for their frameworks for the superior performance of experts. In summary, comparison of experts and novices might have an impact on establishing a scope and limitations of a new domain. By using the novice group as a reference point, it might be more possible to merely understand factors that enable becoming an expert. This is very similar to experimental research in which there are treatment and control groups. 
Control groups get involved in the research in order to demonstrate the mere impact of the treatment on participants if there is a significant expected change.

The combination of both implications. Alruwaii and Brooks (2008) created a map of organizational security by identifying the most relevant categories, and they used multidimensional scaling to analyze the similarities of these categories derived from experts in the security field. In their paper, they emphasized that they conducted the study in order to provide a better understanding of organizational security categories. Moreover, they would like to create an aid for the curriculum of the institutions. They also contended that the framework that they developed in the study would be a reference model to teach proper knowledge and skills to become security professionals in the industry. Another study by Ross, Battaglia, Phillips, Domeshek, and Lussier (2003) applied mental model theory to identify a framework for tactical skills domain; furthermore, they utilized this model as a baseline for developing an effective and efficient training program. They conducted the study while they were observing the sessions in which experts were tutoring novices. The study demonstrates a combination of defining a domain and training and teaching implications of expert and novice comparison studies. These two studies demonstrate not only the stand-alone but also composite consequences of studying expertise on defining a domain and improving professional training.

The concept of mental model has been discussed in different disciplines for several decades since it is a theory to explain how knowledge or information is organized. In expertise research, scholars also have taken mental models into 
consideration. Mostly, the research has been applied to reveal knowledge of experts and novices. The next section briefly explains mental models.

\section{Mental Models: A Means to Reveal Expert Knowledge}

Ericsson and Charness (1994) indicate that reasoning, planning and evaluation skills of experts are vitally dependent on their internal representations about the pertinent information in circumstances. Winn (2004) identifies these internal representations as automaticity and knowledge organization that are two cognitive processes accounting for attaining expertise. Dissimilar to novices, experts do the things without specifically thinking about them. However, both experts and novices do the things the way they learned. He further contends that "if automaticity corresponds to the cognitive process side of expertise . . knowledge organization is the equivalent of mental representation of knowledge ..." (p. 93). Knowledge organization of experts is qualitatively different from novices. They usually search for chunking of information in problem situations; moreover, they take advantage of these chunks by perceiving information more efficiently and retrieve it in a short time, which fosters automaticity. One of the most solid theories to explain knowledge structures is the mental model.

Mental model is a ". . . putative structure that contains knowledge of the world." (Winn, 2004, p. 90), and humankind constantly construct working mental models to enrich the understanding of the world (Johnson-Laird, 1983). A functional and more detailed definition by Rouse and Morris (1985) states that "mental models are the mechanisms whereby humans are able to generate descriptions of system purpose and form, explanations of system functioning and observed system states, and predictions of future system states." (p. 7). That is, people describe, explain and predict events that 
occur in their environment by the assistance of mental models (Mathieu, Heffner, Goodwin, Salas, \& Cannon-Bowers, 2000). For professionals, mental models are one of the most critical characteristics to do their routine job. For example, Mathieu, et al. (2000) indicated that the similarity of individual's mental models working in a team had an impact of team processes and performance. This is an interesting result since the current professional world greatly supports teamwork. Smith-Jentsch, Campbell, Milanovich, and Reynolds (2001) found that high-ranked navy personnel possessed teamwork mental models that were more parallel to the model, which was derived from the expert team, than those who were low-ranked. This study represents evidence between expert mental models and performance. Mental models are also very critical for companies in the current competitive business world. Osborne, Stubbart, \& Ramaprasad (2001) studied the relationship between the common mental models of strategic groups, which is a collection of companies executing similar strategies, derived from the annual reports of the company leaders and the performances of these companies; they found that the combined mental models of the leaders in the strategic groups had a prominent impact on the performances. Mental models play very critical roles in the professional world from individual to organization level when professionals perform; as a result, measuring the most appropriate mental model becomes a serious concern.

Like aforementioned studies, researchers attempted to measure mental models by using different methods in any other diverse studies. Every method cannot supersede one to another, and they have both strengths and weaknesses. The most commonly used methods in expertise research are: (a) interviews, such as 
unstructured, structured, one to one, and semi-structured (Hmelo-Silver \& Pfeffer, 2004; Means \& Voss, 1985; Nelson, 1989; Payne, 1991), (b) card-sorting (Chi \& Koeske, 1983; Hodgkinson, 2002; Smith-Jentsch, et al., 2001), (c) thinking-aloud protocol (Boshuizen \& Schmidt, 1992; Ericsson \& Charness, 1994; Zahodnic, 2009), (d) analysis and completion of tasks and/or cases (Feltovich, et al., 1984; Nelson, 1989), (e) text comprehension (Burkhardt, Détienne, \& Wiedenbeck, 1997), and (f) analysis of relatedness or proximity of concepts, such as link-weighted, that is, Pathfinder networks (Goldsmith \& Johnson, 1990; Schvaneveldt, et al., 1985; Taricani \& Clariana, 2006; Villachica, et al., 2001), and multidimensional scaling (Bradley, et al., 2006; McKeithen, et al., 1981; Schvaneveldt, et al., 1985). Rowe and Cooke (1995) studied a comparison of the effectiveness of three techniques, structured interviewing, related rating, and think-aloud protocol, on trouble-shooting performance related to airborne electronic equipment, and they found that relatedness rating via using PathFinder was the most effective technique. The present study will utilize link-weighted - Pathfinder technique to elicit mental models of participants.

Expertise research has significant impact on the development of a domain since it identifies how experts act. Therefore, investigating the inside of experts' heads in terms of processing and organizing knowledge is one of the most reasonable approaches. Mental model theory provides both knowledge-base and methodology to seek the knowledge organization part of human mind. This functional association of expertise and mental models illuminates the purpose of the present proposed study.

\section{Purpose of the Study}


The major strength of HPT is that most of the experts are practitioners and their knowledge and skills are mostly derived from informal education. This means that there is an enormous amount of undiscovered phenomena and experiences that might contribute to the field's current knowledge base. However, there has not been sufficient research effort directly targeted to the field to collect and form these phenomena and experiences in a scholarly and professional manner. Thus, investigating expert practitioners and their characteristics would provide theoretical as well as practical foundations and advance escalation of the field. This affects not only the domains of the field that are still not completely defined but also the degree programs in the field.

For the aforementioned reasons, the present study concentrated on the practitioners in HPT field. The mental models of expertise of these practitioners were revealed by using the Pathfinder analysis technique. Each participant's mental model enables the researchers to calculate several measures, relatedness, coherence, and similarity (See Definitions section at the end of this chapter for further details), derived from Pathfinder. These three measures are utilized to quantify the mental model of expertise of the practitioners. Next, the relations between the professional profile characteristics of these practitioners and their mental models of expertise were investigated. The outcomes of the present study were a concise understanding of the field, the general characteristics of experts and novices' mental models, and the professional activities that had influenced the mental model of expertise related to general understanding of HPT. Another underlying reason for the present study was to fulfill one of the research priorities (Huglin, et al., 2007). HPT requires empirical studies 
to improve the current theory and practices. The present study was an opportunity for this.

The current research prepared under the motivation of focusing on an expert versus novice comparison study type. The primary purpose of this study is to reveal performance improvement practitioner expert and novice mental models and identify differences and similarities between these models. The secondary purpose is to analyze the potential impact of the professional profile characteristics of performance improvement practitioners on their mental model of expertise. The following research questions were addressed:

1. What is the common mental model of expert performance improvement practitioners?

2. What is the common mental model of novice performance improvement practitioners?

3. What, if any, are the similarities and differences between the common mental models of experts and novices?

4. What are the professional profile characteristics of performance improvement practitioners?

5. To what extent are the professional profile characteristics of performance improvement practitioners associated with their mental models of expertise derived from Pathfinder?

\section{Significance of the Study}

The primary responsibility of researchers regarding expertise research is to demonstrate how experts' superior performance happens (Ericsson \& Charness, 1994). 
Witucki (2006) suggested that further efforts to understand underlying principles of experts performance in HPT might bring different insights to the field, such as development of measurement and evaluation model for expertise, which can be utilized by other empirical studies, and identification of how HPT expertise is developed and applied. He also questioned the effect of formal education and the level of HPT expertise.

Villachica,, et al..'s (2001) domain expertise study on the cognitive map of HPT is one of the initial efforts for expertise research in HPT. The authors recommended the replication of their studies with a larger sample size and condensed version of the questionnaire since they indicated that these two factors hindered the importance of their results. They also emphasized the possible connection between expert mental models and competency creation. Since there are numerous efforts regarding competencies in the field (Chow, 2010; Fox \& Klein, 2003; Giberson, 2010; Guerra, 2003; Lauer, 2008; Vadivelu \& Klein, 2008), the possibility of the connection between expertise and competencies may add more significance to this study.

Winn (2004) discussed that change in mental representation during the development of expertise has not taken adequate interest by educational technologists. Dominant part of theories and principles typically concentrate on the representation of expert mental models that are expected to be acquired at the end of a designed instruction. He further recommends that scholars in the field should devote themselves to understanding the progression from novice to expert. Winn's (2004) perspective must be considered for two reasons. First, the researchers should spend extra efforts to understand dynamics of the process of becoming expert in terms of mental model 
representations that the advanced degree programs must lead their students to construct. Second, the mental models help novices to understand principles, rules, theories and practices utilized currently in the field.

Rowe and Cooke (1995) claim that when the relationship between knowledge representations or mental models and performance is identified, there can be an opportunity of added-value for design, development and evaluation of training interventions in organizations. Their claim shed an insight for expertise research in HPT because the relationship between mental models of HPT experts and their performance might provide empirical rationale for the implementation of all interventions currently used in addition to training. Both the mental models and performance of experts in HPT need to be investigated separately to understand their relationship.

The results of the current study are especially significant and contributing to more precise understanding of HPT expertise. It encourages changes in training of practitioners and scholars as well as changes in degree programs in the field and the way the field is practiced. Human performance technology as a relatively emerging field needs to consider the current experts' network. This effort would be helpful to make the field more distinguishable from other fields in terms of its knowledge base.

\section{Definition of Terms}

Expert: An expert who is an individual possesses ". . . a large body of knowledge and procedural skills" (Chi, et al., 1982) and " . . . performing at least two standard deviations above the mean level in the population ..." (Ericsson \& Charness, 1994, p. 731). Hoffman (1998) defined experts are the individuals who are well respected by other professionals, frequently make accurate and robust decisions, perform very 
effectively and efficiently, cope with unusual and difficult cases successfully, and have the knowledge, skills and abilities stemmed from widespread experiences with subdomains. They demonstrate exceptional performance since they can process information very rapidly, which is due to automaticity, and have hierarchical knowledge structures, which are the collection and connection of effective information chunks (Winn, 2004). They analyze situations more qualitatively, and they have broader perspectives. They focus on fundamental principles and procedural solutions.

Since experts and expertise changes one domain to another, the terms of expertise and expert cannot be identified precisely (Chi, 2006). It is very critical for the scholars to identify the experts clearly when they are conducting research about expertise. In the present study, previously used criteria by Huglin, et al. (2007) and identified by Ericsson \& Charness (1994), and an additional criteria decided by the researcher were used to choose experts in HPT as follows:

1. those who had written extensively in the field of performance improvement,

2. those who have been active in the professional organizations, such as International Society for Performance Improvement (ISPI), The American Society for Training and Development (ASTD), or Association for Educational Communication and Technology's (AECT) Training and Performance Improvement track,

3. those who were recommended by persons identified via the first two criteria, and 
4. those who have had experience in performance improvement field for ten or more years.

5. those who had completed numerous HPT related projects.

Expertise: "Expertise refers to the characteristics, skills, and knowledge that distinguish experts from novices and less experienced people." (Ericsson, 2006, p. 3).

Novice: "Novice is someone who is new - a probationary member [and] has been some minimal exposure to the domain." (Hoffman, 1998, p. 84). Novices apply standard procedures or models which were taught to them. They concentrate on surface features of the problems and they utilize declarative knowledge.

In the current study, novices were selected based on the same five criteria applied for the experts. The professionals who participated in the study were analyzed and those who did not meet the five criteria were selected as novice.

Knowledge Network Organization Tool: It is a software tool built on Pathfinder network generation algorithm (Interlink, n.d.).

Pathfinder: Pathfinder is a collection of algorithms which take estimates of the proximities between pairs of items as input and identify a network representation of these items, which is called PFNet (Interlink, n.d.).

PFNet: PFNet is a network consists of the items as nodes and a set of links connecting pairs of the nodes (Interlink, n.d.). The PFNet networks may be either directed or undirected for symmetrical or non-symmetrical proximity estimates.

Mental Models: "Mental models are the mechanisms whereby humans are able to generate descriptions of system purpose and form, explanations of system functioning and observed system states, and predictions of future system states." 
(Rouse \& Morris, 1985, p. 7). Mental models are crucial since they simplify interpretation and sustainability of information (Gardiner \& Christie, 1987). Awareness of mental models somehow provides individuals an opportunity to control their experiences and competency on specific tasks (Jih \& Reeves, 1992). They also have an impact on factors affecting performance, such as committed efforts, expectations, satisfaction, and prediction of results.

Relatedness Measure: It is a Pathfinder derived measure; it is a PearsonProduct Moment correlation value between sets of concept ratings. It theoretically ranges from minus one to plus one (Villachica, 1999).

Coherence Measure: It is a Pathfinder derived measure; it is a Pearson-Product Moment correlation indicating internal consistency of ratings with an individual's or group's sets of concept ratings. It theoretically ranges from minus one to plus one (Villachica, 1999).

Similarity Measure: It is a Pathfinder derived measure; it is the number of links in common divided by the total number of unique links in the two networks. Two identical networks will yield a similarity of one and two networks that share no links will yield similarity of zero (Villachica, 1999).

The Mental Model of Expertise: It is a quantified version of the mental models of the experts and the practitioners participated in the study. It is the total value of relatedness, coherence, and similarity scores of each individual's mental model.

Deliberate Practice: Deliberate practice, which requires considerable, precise, and continuous efforts to do something one is not good at partially or at all, is a specific practice necessary to develop expertise in a domain (Ericsson, et al., 2007). Deliberate 
practice focuses on improving the current skills and advancing the accomplishments and boundaries of the skills. Deliberate practice, which is necessary more than ten years, is the only empirically proven factor that can predict expertise (Ericsson, et al., 1993).

\section{Summary}

As a comparatively new and emerging field, HPT needs a body of literature and research. In order to improve this, an integration of practice and professional training of HPT must be in place. HPT practitioners, who usually represent the practice side, have been actively producing solutions for decades in organizations; higher education programs, which usually represent the theory side, provide HPT education and training as entire programs or separate courses offered in a performance track. This research, built on expert and novice mental model comparison, attempted to add a conceptual framework to the growing body of HPT literature and research, which is expected to have potential implications for a deeper understanding of the field and for improvement in degree programs and practices. 


\section{CHAPTER 2}

\section{REVIEW OF LITERATURE}

\section{Introduction}

The relevant literature regarding this proposed study is examined in this chapter. The chapter has six main parts: mental models, expertise in domains, relationship of expertise with individual characteristics, weaknesses of expertise, Pathfinder scaling technique, and the conclusion. These areas serve as foundation for the research.

The primary purpose of this study is to reveal HPT expert and novice mental models and identify differences and similarities between these models. The secondary purpose is to analyze the potential impact of the professional profile characteristics of HPT practitioners on their mental model of expertise.

The first section explains an overview of mental model theory, the nature of mental model research, certain evidences for the existence and implications of mental models, and the relationship of mental models with the concept of expertise.

\section{Mental Models}

People are in constant interaction with their environment, other people, and artifacts of technology or objects; as a result, they form mental models, which have predictive and illuminating power of understanding the interaction of these surroundings (Norman, 1983). Mental models must be considered in four different notions: the target system, the conceptual model of that target system, the user's mental models of the target system, and the scientist's conceptualization of that mental model. The target system is how the person is learning, using, or performing. The conceptual model, which is usually invented by teachers, designers, scientists, and engineers, is the 
appropriate representation of the target system. Mental models are the representation of the target system in people's mind. These models are evolving, incomplete, not completely accurate. They are influenced by people's individual differences, background, previous experiences, etc. The scientist's conceptualization of a mental model is a model of a model, which brings all commonalities to attempt to develop, as much as possible, an accurate model. Norman (1983) shared his observations about mental models. First, mental models are incomplete; second, people are incapable to run their model completely; third, mental models are unstable; fourth, mental models do not have distinct boundaries; fifth, mental models are not scientific; models are economical. Norman's (1983) point of view regarding mental models represents their limitations. On the other hand, Johnson-Laird (1980) identifies the several characteristics of mental models. Mental models are the representation of a state of affairs; their structures reflect the relevant aspects of the corresponding state of affairs in the world. The reflection of the structure is direct representation or analogy. Mental models may have multi-dimensions and be dynamic; therefore, they provide the advantage of scanning to any directions in the case of a problem. In other words, they provide a holistic approach in which one can search for all regular or irregular, relevant or irrelevant possibilities. Johnson-Laird (1980) indicates that mental models can be constructed either verbally or perceptually. The components of mental models produce the images of perceptible equivalent real-world objects. They may ensure thought processes occurring without any certain consciousness and trigger the perception of objects by providing prototypical information. 
In his later writing, Johnson-Laird (1983) comprehensively explained the major functions of mental models:

“. . mental models play a central and unifying role in representing objects, states of affairs, sequences of events, the way the world is, and the social and psychological actions of daily life. They enable ... to make inferences and predictions, to understand phenomena, to decide what action to take and control its execution, ... to experience events by proxy; they allow language to be used to create representations comparable to ... direct acquaintance with the world; . .. they relate words to the world by way of conception and perception." (p. 397) Moreover, Johnson-Laird (1983) explains the nature of mental models as several constraints, similar to Norman's (1983) approach, that make them more understandable. Since mental models are in people's head, there cannot be an exact account and explanation. These constraints are listed as follows:

1. Mental models are computable; they provide opportunities to measure.

2. Mental models are finite in size; they cannot represent an infinite domain.

3. Mental models are made up of token or clues in a specific structure to represent a state of affairs.

4. Mental models are economic; a single mental mode represents a single state of affairs regardless of its completeness or indeterminacy.

5. The complexity of mental models does not develop exponentially.

6. Mental models contain concepts natural to them; they have the capability of examining predictability of concepts within a set.

7. Mental models are constructed by innate conceptual primitives, which are the fundamentals of concept. 
8. Mental models are a finite number of common conceptual primitive sets; they are also the part of further finite sets. This ensures the increment of complexity of mental models based on underlying primitives.

9. Mental model structures are identical to the structures of the state of affairs which are represented.

10. Mental models are formed by hierarchical sets where all members are identified.

Mental model theory is highly significant for human-kind. For this reason, researching this theory might have a potential to reveal human-kind's undiscovered inherent capacities and processes. Gentner \& Stevens (1983) state that fundamental concern of mental model research is to understand, specifically, people's understanding some domain of knowledge and, broadly, human knowledge about the world. There are three dimensions that characterize mental model research: the nature of the domain that is studied, the nature of the theoretical approach, and the nature of the methodology. The following section explains these three dimensions and then provides research that shows existence and implications of mental models.

The nature of domain. Rouse and Morris (1986) identified a model to demonstrate the distinction of mental model theory among domains since they believed that the mental model concept was studied in diverse domains that resulted in ubiquitous understanding. They identified two dimensions: the nature of the model manipulation and the level of behavioral discretion to explain wide-ranging differences about mental models. The nature of the model manipulation elucidates whether individuals are aware of their own manipulation of mental models, and it ranges from 
implicit to explicit; the level of behavioral discretion, which ranges from none to full, explicates whether an individual's behavior is a matter of choice or dictated by task(s). The mental models of a domain can be classified based on these two dimensions. The framework also depicts methodological differences among domains: (a) inferential and (b) verbalization methods. Inferential methods are prone to reveal more accurate representation of mental models when there is a little discretion; verbalization methods attempt to provide more appropriate representation of mental models when there is an explicit manipulation. Verbalization methods produce more subjective mental models; whereas, inferential methods produce more objective results.

The nature of theoretical approach. The nature of the theoretical approach causes different perceptions of mental models; as a result, there are numerous other terms for mental models, such as cognitive structures, knowledge structures, cognitive maps, schemata, etc. Schemata and mental models usually are used interchangeably (Winn, 2004). However, mental models have a broader nature than schemata since they contain not only objects but also their casual relations, and, of course, numerous scholars disagree with this thought. Multimedia learning usually considers this distinction between these two concepts. Tversky (1993) discussed that cognitive maps are not as effective as cognitive collages and spatial mental models to remember an environment and its components. According to the author's argument, cognitive maps preserve metrics and characteristics of sub-components of an environment; cognitive collages preserve a general overview of the environment without details about metrics and sub-components; spatial mental models preserve coherent relations between subcomponents and the whole as a picture of the environment. At the end of the 
experiment regarding the retention of large scale maps by utilizing these three different models, Tversky indicated that when people knew and learned the environment very well, they developed a spatial mental model that facilitates inference and perspective taking. This study demonstrated that mental models can handle more complex knowledge and information structures than cognitive maps and collages. In the complicated environments, individuals might utilize, manage, and improve their mental models as what experts do in their domains. This is another understanding of mental models from cognitive science theory. There might be similar types of comparisons in the literature. Even though there are numerous term names, they explain the same or very similar phenomenon; therefore, they are known as synonyms of each other (Beier, Campbell, \& Crook, 2010). The researcher of this present study accepts all these different terms as names for mental models.

The nature of methodology. For methodological dimension, identifying how to elicit mental models might decrease the complexity of the concept. Jonassen (1995) seconds the aforementioned perspectives that mental models are dynamic, multimodal, multidimensional, complex, inherently epistemic constructs that are more than structural maps. They help individuals establish a beginning for how we know what we know. In addition, he believed the major approach to reveal a mental model is performance. He further discussed that due to the nature of mental models, they require multiple data sources to be examined; as a result, he identified several characteristics as criteria to assess mental models, which are coherence, purpose or personal relevance, integration, fidelity with real world, imagery, complexity, applicability or transferability, inferential or implicational ability. 
Cooke (1994) summarized the dimensions of knowledge elicitation techniques, which is a good framework for a brief overview. She classified these techniques in a continuum between informal, e.g., observations, and formal , e.g., conceptual. In the informal techniques, the elicitor role is active; experts respond directly; timing of elicitation is concurrent; collected data are qualitative. On the contrary, in the formal techniques, elicitor is passive; experts' responses are indirect; elicitation occurs separately; quantitative data are collected. She further discussed the strengths and weaknesses of each technique class. Elicitors need to have interviewing skills and domain knowledge when informal techniques are used; whereas, for formal techniques, procedural and analytical details of these techniques must be understood. Informal techniques entail introspection and verbal reports; formal techniques require devoted time for material preparation. The results revealed in informal techniques are inferences from the task analyzed and limited to this task. On the other hand, the formal techniques produce more general results, but these results are artificial and might lack face validity. The informal techniques provide rich data; however, it is time-consuming to analyze and interpret these data. The formal techniques have an opportunity to collect data from more participants and analysis of group data. Pathfinder scaling algorithm is classified as formal techniques. In the last section of this literature review, the validity and sample studies of Pathfinder scaling are presented.

Carley and Palmquist (1992) developed a framework to extract, represent, and analyzing mental models. Their model is innovative since they established it as an alternative to the previous techniques, e.g., content analysis, procedural mapping, task analysis, and cognitive mapping, and they discussed both strengths and weaknesses. 
Their framework contains four components: (a) concepts, (b) relationships, (c) statements, and (d) maps. Statements are the most important component since they are formed by two concepts and the relationship between them. The collections of statements via more relationships compose the map. The process of obtaining the mental model starts with the identification of concepts relevant to the topic under investigation. Second, the relationships between concepts are defined; this also reveals statements. Finally, all statements and concepts are connected to each other, which provide a map that is a mental model of an individual regarding to the topic.

The existence and impacts of mental models. Mental models are very popular topics for the science world. In this section, the variety of studies that demonstrate the existence and impact of mental models is presented. Payne (1991) conducted an experiment regarding people's beliefs about high-street bank machines to identify the phenomenon of mental models from the cognitive science perspective. His conclusion depicted certain information about mental models. Individuals had already a mental representation of bank-machines, and, interestingly, they started to speculate it during the experiment. When they were asked to talk about the system, they always attempted to explain it with models that they generated for other familiar domains. When they had a claim about the system, they were prone to support it by the first-hand experiences. These results are critical to understand the basic features of mental models. First, every individual has a mental model of any topic whether it is accurate or not. Under the circumstances of explicit manipulations, such as teaching, mental models are inclined to change. Finally, mental models play a critical role on one's decision regarding performance since mental models creates a basis for the action. 
Gray (1990) utilized protocol analyses and drawing techniques to study mental model construction during hypertext navigation. She specifically focused on potential difficulties of linear book metaphor on naive users when they used a hypertext system. She found that even though this metaphor hindered users initially, they eventually got accustomed to a hypertext system. The result of this study was interpreted by the author for the implications of human-computer systems design; on the other hand, it has another important consequence that it confirms the dynamic and incomplete characteristics of mental models. In the case of novel situations, individuals can seek the closest part of their mental models and utilize it even though this part contradicts the current situation.

The relationship between mental model and learning is one of the commonly studied topics since these two concepts mutually influence each other. Mental models or activation of mental models are accepted as effective tools for meaningful learning; moreover, when an individual learns something new, one way to explain this new acquired knowledge is changes in the mental model. Examples are presented for each of these relations. First, Kieras and Bovair (1984) studied the effect of mental models on learning how to operate a device. They applied three different experiments, which utilized a device model and rote learning groups. In the first experiment, they found that the device model group learned a set of procedures about the device faster and retained more knowledge than the rote learning group. The second experiment demonstrated that the device model group inferred model procedures better than the second group. Finally, the third experiment analyzed what types of information had a critical impact on learning. It revealed that specific information regarding the controls 
and their role within the overall system was the most critical information rather than motivational and general explanation of each system components in the whole system.

Second, Ifenthaler, Masduki, and Seel (2009) performed a study to analyze the development of cognitive structures during instruction based on graph theory, which assumes that a graph is made up of vertices whose relations demonstrated by edges, and the basic dynamics between vertices and edges provide an opportunity to examine graphs quantitatively. They identified two features of cognitive structure: organization and semantic content. Organization of cognitive structures are constructed by surface structure (the number of propositions as node-link-node form), graphical structure (to what extent the subject matter is understood), connectedness (the deep subject matter understanding), ruggedness (non-linked vertices showing less understanding), average degree of vertices (the number of incoming and outgoing edges), cyclic (the degree of complexity), number of cycles (the degree of association between vertices and edges), and vertices (the degree of the size). Semantic content is presented by vertex matching (the use of semantically correct concepts or vertices) and propositional matching (the use of correct proposition or vertex-edge-vertex relationship). The study results indicated that there was a significant change in the organizational feature of the participants' cognitive structures, except for the average degree of vertices. When the participants became familiar with the subject matter, they produced more and more complex structures. On the other hand, a significant increase of ruggedness and decrease of connectedness indicated that the new concepts were not incorporated into the cognitive structures instantly. Second, the number of semantic correct vertices of the participants' cognitive structures became similar to expert structures; however, the 
same result was not observed for the number of semantic correct propositions, which might need more hands-on experiences.

The aforementioned studies are limited to the nature of mental models, their effects, and their development via learning. In broader sense, according to Rouse and Morris (1985), the mental model research should focus on substantial issues, such as accessibility to models, form and content of representations, nature of expertise, cues, which foster mental model improvement, utilization, and instructional concerns. Their perspective highlights the significant necessity of mental models to understand nature of expertise. Interestingly, Winn (2004) criticized that the professionals in HPT or ID fields assume that task analysis should contain behaviors or mental representations of experts to develop programs to train novices. He further argues that rather than this assumption, to understand the progress from novice to expert might illuminate more valuable information for the field. The dynamics and mechanism of this progress is worthy to investigate. According to Ross, Shafer, and Klein (2006), expertise development starts with searching information that leads to the learning of detailed patterns, and then the categories in the mental models are revised. After this, new courses of actions are created and sustained.

It is obvious that expertise and mental models are integrated concepts, and mental models are critical to reveal the underlying reasons of expert performance. For this reason, Serfaty, MacMillan, Entin, and Entin (1997) established a mental model theory for studying expertise and examined it in an experimental environment. They called it a three-stage hourglass model including recognition, exploration, and matching of the process by which mental models are developed and used by the experts. Experts 
used both inductive and deductive processes to utilize this model. The first stage, recognition, includes expert's generation of mental models regarding situation and identification of potential solutions. The deliverable of the recognition is initial plan. The whole process is an inductive reasoning. The second stage, exploration, covers expert's questioning process, and it reveals a developed plan. This stage is deductive, unlike recognition stage. In the final stage, matching, expert starts harmonizing the developed plan against mission or task using improved mental models and envisioning their effectiveness. The study that was performed to confirm the framework was conducted on 46 military officers ranked from Captain to General. The subjects presented a battlefield scenario, and they were asked to respond to the most appropriate decision. The expertise level of subjects was measured based on judges and behavioral components including theoretically expected behaviors from an expert in the task situation. The following results derived from the study:

1. The more and less expert subjects did not provide an initial course of action quickly.

2. The subjects with high expertise provided more detailed initial course of action than those who were less experts.

3. The high-expertise subjects suddenly focused on critical unknowns and asked right questions for potential solutions.

4. The expert subjects in the study built and used richer mental models than the less-expert subjects, and they also used these models for envisioning the consequences of their decisions. 
5. The expert subjects constantly emphasized their concerns regarding the consequences of a decision. This supports that experts used their mental models to visualize the potential outcomes.

6. The visualization of the further results, which was applied by experts, increased the quality and flexibility of their course of actions. The presence of contingences in the developed plan and amount of changes that had been already planned in course of actions demonstrated evidence in the study.

The study also revealed that mental models were one of the reasons for experts' superiority since experts used them to understand, investigate, and foresee a problem situation holistically. Experts also took advantage of mental models by making progressive connection between each stage.

Even though expertise is perceived and used as a general term, experts demonstrate their performance in real-life settings and different domains. Due to the differences of subject matter, there should be some differences on the experts and expertise. The following section provides details about expertise in diverse domains.

\section{Expertise in Domains}

This section explains how expertise is observed in different domains. There are four common approaches for this: (a) identifying what types of knowledge, skills, and abilities that experts should have, (b) clarifying what types of cognitive processes help experts perform exceptionally, (c) defining how expert knowledge is organized, and (d) revealing what types of similarities and differences exist between experts and novices. The section will provide a general understanding of expertise, and then expertise in health sciences, language skills, soft skills, e.g., decision making, situation awareness, 
self-regulation, etc., technical/operator skills, chess, logical/mathematical skills, physics, historical/political sciences, teaching/learning, and human performance technology.

Expertise in general. After extensive review, Sternberg (1997) identifies nine different views of expertise: (1) the general process view in which experts are advanced and rapid problem solvers by using the same or different techniques that non-experts usually cannot use; (2) the quantity knowledge view in which experts have more knowledge than non-experts; (3) the knowledge organization view in which experts organize their knowledge more effectively than non-experts; (4) superior analytical ability in solving problems, which indicates effective usage of knowledge; (5) superior creative ability, which indicates creation of knowledge based on the existing knowledge; (6) superior automation, which indicates that experts do things more unconsciously and smoothly; (7) superior practical ability, which indicates that experts know better how to start and begin a task in an effective and efficient way; (8) people's conception of expertise, which indicates an individual's label as an expert by other people; and (9) the final one is the combination of these eight views as a prototype, which indicates expertise is rarely reached in its pure form. This classification explains general common characteristics regardless of domains where experts might show different characteristics.

Honeck and Temple (1992) created a tetrahedron metaphor in order to illuminate research on the cognition of expertise, which can also explain expertise in a broader sense; and they identified four of the main indicators, which were located at the each corner of the tetrahedron: the features of the Problem, e.g., complexity and familiarity, the Expertise level of research participants, the social Situation where the problem 
needs to be solved, e.g., individual or groups, and the Task used to reveal knowledge, e.g., think-aloud protocol, card-sorting, or concepts maps. The model is also called PEST. Even though the model is comprehensive, it does not emphasize a complete picture of the contextual factors. Hence, experts usually show their expertise in real-life settings where there are ill-defined and under pressure situations. In order to eliminate this discrepancy, Hoffman, Feltovich, and Ford (1997) modified the model and named it as the elaborated and modified PEST model (TEMPEST) by adding several forces to the existing model. The TEMPEST model includes: (a) The kite, the task engagement of the expert, is the modified version of the PEST model. The expert engages in a task by considering background experiences, goals, materials and strategies, which are located at the each corner of the tetrahedron, (b) the line, the controlling forces, includes methods to reduce risks, situation awareness, and moving between diverse methods, (c) the wind, the driving forces, is the collection of social expectations and needs, and (d) the tail, the stabilizing forces, contains training methods, professional standards, selection or competency standards.

Health sciences. Expertise in medicine and surgery is the combination of analytical and experiential knowledge (Norman, Eva, Brooks, \& Hamstra, 2006). Dissimilar to other fields, neither a large set of examples encountered through diverse experiences nor general skills that can be useful for musical skills, such as playing violin, are not sufficient to explain medical and surgical expertise standalone. The knowledge and the cognitive processes generated from this knowledge are the key player in medical and surgical expertise. Johnson, Durán, Hassebrock, Moller, Prietula, Feltovich, and Swanson's (1981) investigation on expertise and error in diagnostic 
reasoning pointed out that the effect of expertise was observed on the only content or substance of reasoning behavior that was identified as problem-solving process rather than the behavior itself. In other words, experts had the declarative knowledge related to the problem solving task, but they had not yet transformed it into procedural knowledge.

An experimental study by Stefanidis, Korndorffer, Markley, Sierra, Heniford, and Scott (2007) confirmed the importance of deliberate practice on expert performance in real-life settings. In the study, the short-term retention and transfer to real operative room skills of medical students were measured after an application of laparoscopic suturing simulator training program. There were two modes of the training simulation; the first mode, which was also called conventional training, provided the training until the proficiency scores on two consecutive plus ten additional attempts were obtained; the second mode provided noise (constrained space, short suture, or dropped needle) in addition to the features of the first mode and dropped ten additional attempts. The study indicated that the increased difficulty level of the simulation only impacted shortterm retention on the simulation, but the operative room performance outcomes were not as similar as those who trained conventionally. However, students who trained in the conventional simulation including ten additional attempts showed the same performance on operative performance with those who did longer and harder training. Even though the fidelity of training tasks were developed as close as possible to the real-life settings where experts perform, they were not influential on the transfer performance. In consequence, the effect of deliberate practice on expertise still remains stand-alone. 
Mitchell and Unsworth (2005) compared the reasoning process of expert and novice community health occupational therapists during their home visits, which were recorded by a camera and analyzed both quantitatively and qualitatively. The study revealed that expert and novice occupational therapists demonstrated many differences in terms of the cognitive and affective dimensions of their reasoning process. Experts used the composite of conditional, procedural, and other types of reasoning processes; whereas, novices preferred merely procedural reasoning. Novices' conversational approach was more dependent on assessment forms to guide the process; in contrast, experts used free-flowing conversational approaches. Experts seemed more confident and clear with their reasoning process. Novices were more awkward and selfconscious. Experts were handling sensitive issues very well while novices tried to avoid them.

A study comparing the performance on diagnosing four different cases of medical students, resident trainees, faculty members, who are identified as experts, demonstrated that experts were distinguished from students and trainees due to their successful diagnosis that was influenced in diagnosticians' disease knowledge, a memory store of disease models and the memory organization among the disease models (Feltovich, et al., 1984).

Language skills. Extensive reading, high verbal skills, effective use of concrete language, and envisioning and responding to the readers' expectations are distinctive characteristics, which are specific to the domain, of professional writing expertise (Kellogg, 2006). Moreover, experts in professional writing demonstrate several common characteristics with other domains, such as musicians and computer programmers. 
Experts display effective usage of working memory to control cognitive load and tenyear deliberate practice rule, which is only empirically proven factor affecting expertise (Ericsson, et al., 1993). An individual can become an expert in a domain if she or he practices deliberately to improve his or her performance.

A study which compared working memory performance of novice and expert interpreters pointed out that there are fundamental differences between two groups (Köpke \& Nespoulous, 2006). Novice interpreters, interestingly, outperformed expert interpreters since novices' working memory capacity is more likely to improve when they are challenged in new conditions; whereas, experts have some other cognitive processes omitting working capacity, such as automaticity (Winn, 2004). The result of this study might explain why novices interpret problems or tasks in terms of features, syntax, words or objects. Novices are more skilled to utilize their working memories which help them to recall information based on superficial characteristics.

Nation and Mclaughlin (1986) studied good language learner concepts from information-processing perspectives by conducting causal-comparative research on multilingual, bilingual, and mono-lingual subjects. Multilingual subjects were defined as experts; bilingual and mono-lingual subjects were defined as less experienced subjects. They found that multilingual subjects learned grammar more superiorly than less experienced subjects in implicit learning; however, the same result was not obtained in explicit learning. They further discussed that multilingual language learners had more experiences related to learning language in natural settings rather than under predefined rules. This study shed insight to an interesting point regarding expertise. Multilingual learners have had extensive experiences about learning different 
languages; therefore, they develop an expertise on language-learning in which the process becomes automatic, and the structure of knowledge is more prone to be remodeled when there is a need to learn a new language. In fact, expertise research resulted in an alternative instructional strategy for language acquisition in this study.

Soft skills. The naturalistic decision making field focuses on understanding expert performance and behaviors under difficult conditions, such as time-pressured and ill-defined contexts (Ross, et al., 2006). Phillips, Klein, and Sieck (2008) indicate several factors influencing expertise like perceptual skills, mental models, sense of typicality and associations, routines, declarative knowledge, mental simulation, assessing the situation, finding leverage points, managing uncertainty, and understanding one's own skills, talents, and so forth. Mental models help experts hold a rich internal representation regarding the dynamics of their domain; as a result, experts can learn and understand circumstances more promptly. Salas, Rosen, and DiazGranados (2010) summarize an extensive review of the mechanism of the performance and development of expertise-based intuitive decision-making. The mechanism of performance is influenced in the characteristic factors that are large and well-organized knowledge-base, pattern recognition, sense-making, situation assessment and problem representation, automaticity, and mental simulation. The development mechanism entails deliberate and guided practice, self-regulation, feedback seeking, motivation, and goal setting. These activities improve and maintain a decision-makers' expertise-based intuition. Salas, et al.'s (2010) framework provides a large scope for examining and evaluating expertise-based intuition. 
Dew, et al. (2009) conducted an empirical study that compared the decision making characteristics of expert and novice entrepreneurs. They found that experts used effectual logic , e.g., identifying more potential markets, perceiving a venture as a whole, paying less attention to predictive information, considering current resources carefully, investing on what can be affordable to lose, and valuing partnership; whereas, novices tended to make decisions in predictive frames that were highly dependent on textbook information. Their study is slightly different from other expertise comparison studies since they categorized differences in terms of general and entrepreneurial expertise. They divided general expertise into analogical reasoning, holistic and conceptual thinking, weighting of predictive information, and intuition and gut feeling. Experts demonstrated superior performance in all dimensions except for weighting of predictive information and intuition and gut feeling. Novices tended more to believe and accept market research than experts. There was no significant difference between two groups for using intuitive and gut feeling information. For entrepreneurial expertise, the authors investigated decisions that were means versus goal-driven, affordable loss versus expected return, and partnership versus competitiveness. Experts were more likely to use means-driven approaches mostly in their previous experiences than novices. Experts considered project affordability; whereas, novices were more likely to get involved in larger expected value conditions. Experts tended more to establish ventures with partnerships than novices who preferred to concentrate on rivals. Moreover, novices were likely to sell their product or services based on approaching customers while experts chose to use their network. 
Endsley (2006) speculates that situation awareness, which is up-to-date understanding of the current world environment, is a prominent basis for experts regardless of different domains. Endsley (1988), in another writing, defined situation awareness in a three-level hierarchical framework, which includes perception, comprehension, and projection. In the first step, an individual perceived the information from the surrounding environment. The next step, the individual understands the meaning and importance of the information that was perceived. In the final stage, the individual attempts to foresee the future situations and events based on the perceived and comprehended information.

Endsley (2006) depicts the role of expertise in situation awareness on the continuum of novice and expert. He explains that situation awareness is highly demanding, regularly incomplete, and inaccurate for novices since they have limited attention and working memory capacity. However, experts can develop situation awareness quickly without spending huge amounts of effort, and their situation awareness is usually complete. They have also high level comprehension and projection. The main reasons for these divergences are schema of prototypical situations, mental models of domains, automaticity of processes, and learned skills, such as scan patterns and communications. After extensively reviewing different studies in expertise and situation awareness, Endsley (2006) concludes that novices have several problems while building situation awareness, such as poor information management strategies, poorly directed information-seeking behaviors and scan patterns, constrained processing of the information that they perceive and understand, and lack of appreciation regarding the importance or meaning of the information. 
Randel, Pugh, and Reed (1996) studied the differences in the situation awareness of expert, intermediate, and novice electronic warfare technicians while performing cognitive tasks wherein the participants made decisions to identify objects whether friendly or hostile. The expertise groups of the participants were identified based on a performance assessment tool. Experts in the study focused on examining the nature of situation; whereas, novices emphasized the course of action. Hence, the superior number of experiences that experts and intermediates had over novices assisted them to examine situations more elaboratively. Novices were prone to taking immediate course of action due to their lack of experiences. Their analyses of the situation were shorter than experts. The authors also analyzed the usage of rules of thumb, and the results show that experts demonstrated superseding performance because of their better knowledge about how to apply rules rather than rules of thumb. The authors discussed that expert performance actually was supported by expert tacit knowledge that was procedural, experiential and acquired through informal education. As a result of the study, the authors indicated that since they identified expertise based on real performance, situation awareness would be one of the diverse factors affecting decision making. Moreover, training of novices on situation awareness would be an effective technique to improve their decision making skills.

Self-regulation can have a positive influence on increasing the effectiveness of knowledge and skills possession (Zimmerman, 2006). It cannot explain the basis of expertise standalone since there are other significant factors, such as knowledge and performance skills. However, it can encourage the motivation as well as persistence of practice in the domain of expertise. Moreover, self-efficacy and confidence of an 
individual, which is critical for novel situations, are the other areas that self-regulation might improve. There are a few promising empirical evidences that demonstrated the aforementioned points related to self-regulation, such as Cleary and Zimmerman's (2001) study on expert, non-expert, and novice basketball players' free shot performance; Kitsantas and Zimmerman's (2002) work related to the forethoughts phase goals, strategy choices, self-efficacy, intrinsic interest, self-reflection, the satisfaction of the women expert, non-expert, and expert volleyball players; Cleary, Zimmerman, and Keating's (2006) research about the accumulative effect of selfregulation training in forethought, performance, and self-reflection phases on the attainment of novel motor skills and self-reflective thinking by 50 college students.

Hmelo-Silver, Marathe, and Liu (2007) studied the differences between experts' and novices' understanding of complex systems, the human respiratory system and an aquarium ecosystem. They analyzed the differences based on structure-behaviorfunction framework. Structure stands for elements of a system; behaviors stand for the mechanisms of each element to achieve outcomes or functions; functions stand for the role of an element in the system. The understanding of behaviors and functions are more complex than the understanding of structures. The result indicated that novices comprehended structures; whereas, experts were more capable of understanding behaviors and functions. Moreover, the authors compared expert mental models. They observed that there were qualitative differences between their representations in terms of their scope. For instance, they utilized a hobbyist and biologist for the aquarium task, and they found that the hobbyist thought in more localized and concrete ways to keep an aquarium healthy; whereas, the biologist had broader and more abstract ideas. They 
further discussed that these differences occurred since the goals of experts were diverse. For instance, the hobbyist had practical goals, but the biologist had more abstract or theoretical level goals.

Technical and operator skills. The behaviors of transportation operators (i.e., drivers, pilots, and traffic supervisors) were clearly explained by their expertise level (Durso \& Dattel, 2006). Mainly, experts in transportation demonstrated a better usage of resources compared to non-experts by applying efficient transitions between tasks, different prioritization strategies of sub-tasks, and explicit devotion of resources to management. The difference between experts and non-experts in transportation derived from the use of various strategies rather than knowing the collection of facts.

Bradley, Paul and Seeman (2006) conducted a study that compared expert and novice technicians' mental models while using a mail sorting machine. They grouped participants based on two different criteria: experience and job performance. When participants were grouped based on experience, expert mental models had few links, were disorganized, and did not differ from the mental model of novices. On the other hand, when they were grouped based on observed job performance, they had mental models with more links, hierarchical structure, and abstract concepts. This study confirms that deliberate practice is the only predictor of expertise (Ericsson, 2008; Ericsson \& Charness, 1994; Ericsson, Krampe, et al., 1993; Ericsson, Prietula, et al., 2007). Hence, experience may not be deliberate practice. However, high performers may utilize deliberate practice either consciously or unconsciously to maintain their exemplary performance levels. 
Hill (2006) compared mental models of professional engineers in music industry with senior students in the music recording program at a four-year college. Structural knowledge was elicited via card sorting and multidimensional scaling; procedural knowledge was elicited by using think-aloud protocol while participants were completing a task. As a result of the study, experts had more sophisticated and highly structured knowledge that helped them adapt to unfamiliar situations.

Computer programming. McKeithen, et al.. (1981) compared the recalling performance of beginner, intermediate, and expert programmers for using a programming language that associates the certain functionalities to the specific words. Beginners disregarded functional significance of the words and utilized very general mnemonics techniques to recall them, such as using the first letter of words. In the intermediate group, recalling was observed in more grouped chunks format, and the recalled words were more general programming language format rather than the specific format of the programming language investigated in the study. This meant that they used their general programming language knowledge to recall the words investigated in the study. Experts showed very similar chunking structures within their group. They recalled words with regards to their functions, and the experts took advantage of this situation so this increased their performance. Adelson (1981) confirmed the previous results that novice computer programmers used a more syntaxbased organization; whereas, the experts used a more abstract hierarchical organization on the basis of program function in an experimental study, which compared the two groups recall performance of three different complete programs. 
Burkhardt, Détienne, and Wiedenbeck (2002) studied the factors, which were expertise, task type, and development of understanding, on program comprehension. Expert programmers were chosen based on their years of experience on programming; there were two tasks: (a) documentation, which requires the documentation development of the programming codes, and (b) reuse, which requires reusing programming codes in a problem situation; there were the two phases of the experiment: (a) study, which subjects only studied the programming code and were not able to modify, and (b) task, which subjects were able to make changes and use it, phases. They analyzed both program and situational programming comprehension models. The program model was text-based understanding of the programming, and the situation model was the condition referred by the text, that is, mental model of the programming code. In documentation task group, expert and novice programmers differed in terms of elaboration of the situational models, but the same result was not true for the program model. In reuse task group, novices improved their situation models from study to task phase. The overall result of the study demonstrated that if tasks demanded the construction of the situational models, novices were able to develop them similar to experts.

Burkhardt, Détienne, and Wiedenbeck (1997) conducted an empirical study to examine the cognitive validity of the distinction between the program model and the situation model in object-oriented program understanding. The program model refers text-based representations of a program. Unlike the program model, the situation model contains entities of a problem domain and their relationships. The situation model is similar to the mental model concept that knowledge is in a structured form by entities 
and relations. There were three critical results of their study. The first result pointed out the superior impact of the situation model over the program model in terms of recalling knowledge for testing. The second demonstrated that expertise had a significant impact on the construction of the situation model; whereas, the program model was not influenced. In addition to these results, the study revealed that the situation models built by experts were stronger than those built by novices.

Chess. Grabner, Stern, and Neubauer (2007) investigated the controversial relationship between individual differences and the attainment of expertise in chess domain. They analyzed the influence of intelligence, personality and emotional competencies, experience in chess play, tournament participation, and practice activities on player strengths, which was identified based on an extensive and robust international ranking system so that it was an indicator of chess expertise. Four intelligences were identified: (a) general, (b) verbal, (c) numerical, and (d) figural. Five personality factors were identified: (a) neuroticism, (b) extraversion, (c) openness to experience, (d) agreeableness, and (e) conscientiousness. Finally, emotional competencies were identified as (a) perceptions of own and other emotions, (b) emotion expression control, (c) masking emotions, and (d) regulations of own and other emotions. The study revealed that chess experience, current tournament activity, numerical intelligence, domain-specific performance motivation, and emotion expression control accounted for more than half of the variability of chess expertise. The strongest factor was chess tournament experiences among the others. The result of this study revealed that expertise in chess was multi-dimensional and could not be explained by experience in chess playing alone. 
An experimental study by Bilalic, McLeod, and Gobet (2008) examined the relationship between expertise and flexibility of experts in the chess field. They found that there was a positive relationship between the level of expertise and flexibility of experts; however, they also provided that ordinary experts, who were individuals performing at two standard deviations level above the mean of a sample, were unable to oppose the persuasion of deciding the well-known solution. On the other hand, super experts, who were performing at five standard deviation levels, had control over their performance by considering all relevant or irrelevant details; therefore, this helped them to resist the persuasion aforementioned. The result of this study is interesting since the results might reveal a meta-cognitive side of expertise. Ordinary experts were not able to consider the potential pitfalls of their expertise, but super experts were aware of them either consciously or unconsciously. They had sufficient experience to think about their expertise meta-cognitively as well. In addition to superior expertise characteristics, e.g., automation, rapid and accurate recalling, complex knowledge structures, effective and efficient use and growth of knowledge structures, meta-cognition about expertise might be another dimension of understanding expertise. According to Clark (2008), metacognitive skills are crucial components of adaptive exercises that "experts must go beyond their schemas developed in long-term memory to structure their experience in new ways." (p. 316). The results in Bilalic, McLeod, and Gobet's (2008) study might be explained with Schraw and Moshman (1995) explanation regarding meta-cognitive theories, which is explicated in a continuum. They are tacit, informal explicit, and formal explicit theories. Tacit theories are an individual's possessed theory that is acquired without any explicit awareness; informal explicit theories are possessed by individuals 
who are aware of the beliefs and assumption of the theory but have not constructed a framework to incorporate and substantiate these beliefs explicitly yet; formal explicit theories are the highly systematized accounts and a solid skeleton similar to theories taught in higher education. Meta-cognition starts with tacit form, and then it transforms itself into informal explicit form, consequently, it turns into formal explicit form. The ordinary experts might be at the level of informal explicit theory stage in terms of their expertise; however, super experts have already reached formal explicit meta-cognition, and they consider every detail because of their solid framework.

Gobet and Charness (2006) examined expertise studies in chess; therefore, they indicated that experts in chess demonstrated a rich network of chess patterns that enabled them to have a larger visual span and encoded chess information more quickly and accurately than novice players. Moreover, chess experts examined important squares on the chessboard and thought about the critical relationship for the next good moves. Interestingly, Charness, Krampe, and Mayr (1996) found in their study about players of chess tournaments that the chess skills of the players were explained by their previous serious stand-alone studying, size of chess library, and current ages, which had a negative impact.

Logical and mathematical reasoning. A study on mathematical problem solving indicated that novices understood the problems by their superficial characteristics, such as words or objects used in the problems; whereas, experts perceived the problems based on their deep structures associating problems with underlying principles or methods (Schoenfeld \& Herrmann, 1982). 
The only field where there is much evidence for superior performance is calculation. Butterworth (2006) claimed that extra enthusiasm and hard work were the main aspects explaining an individual's exceptional performance for calculation. However, even though he mentioned the low impact of capacity on superior performance, he discussed that an individual's capacity, disposition or other innate abilities might affect the motivational level and be willing to spend more effort. His point is a different perspective because he questions the effect of individual differences as antecedent and indirect aspects of the aforementioned factors influencing eminent performance rather than focusing their direct impact.

Physics. Four experimental studies on solving physics problems speculated that when experts confronted a problem, they spend time to perform qualitative analysis of the problem situation (Chi, Feltovich, \& Glaser, 1981). Experts, first, focused on cues that were utilized to activate the current schemata. Second, they tested the selected schemata for its appropriateness to the problem. Once they were convinced about the schemata, they confirmed it and provided further steps of the solution. Experts understood the problem statement in terms of categories that were defined by the major principles; whereas, novices interpreted it as surface features included in the problem. During a problem solving process, experts' schemata demonstrated a large amount of procedural knowledge which also could be applicable to diverse contexts. On the other hand, novice schemata showed sufficient concrete declarative knowledge about the physical situation of the problem and deficient abstract information.

Kohl and Finkelstein (2008) conducted a causal-comparative study in order to observe similarities and differences between experts and novices in terms of using 
multiple external representations while solving physics problems. They found that experts superseded novices by solving problems quickly and switching between the representations in fast progression. However, they indicated that the extensive use of representations were the same in both groups, which they did not expect. They stated that both groups used the same amount of representations and the same types of problem features. Nevertheless, they added that even though the usage of representations was the same, the time the participants spent for representations were different in terms of the problem stage that participants utilized. Experts devoted more time on analysis and exploration stages; they also focused on identifying goals and subgoals and proceed based on these goals; in contrast, novices did not spend the same time for the aforementioned stages, and they did not guide their solution process with clear purposes. Moreover, the novices hoped that they were accurate in the solution.

Historical and political sciences. In disciplines of history or political science, since problems are ill-defined, and the processes to resolve these problems cannot be controlled as in well-structured experimentations, solutions are mostly arguments rather than precise evidences (Voss \& Wiley, 2006). Thus, study of expertise becomes more complicated than in a field like physics. Of course, there are several factors that influence expertise in history. The first factor is identified as subject-matter knowledge in the field of expertise. However, Voss \& Wiley (2006) discussed that there was not adequate effort to understand the development of subject-matter knowledge in both history and other domains. They further suggest that even though time seems the most impactful factor on history expertise, it is not sufficient to explain all dynamics of expertise so the learning and organization of knowledge of experts might bring more 
meanings. They indicate that expertise in history emerges in graduate school, and a defense of a position, using counterfactuals, and historical analysis techniques development are the part of history expertise.

Fiske, Kinder, and Larter (1983) performed an experiment about the knowledge strategies use of experts and novices in political cognition. They hypothesized that the experts utilized information that was inconsistent with their prior knowledge in addition to consistent information; however, the same situation was not true for the novices. They found that the novices especially focused on consistent information. The amount of consistent information surpassed the amount of inconsistent information. Finally, novices favored the recall of consistent information more than inconsistent information. Experts, in contrast to novices, focused their attention on inconsistent information. The authors indicated that the reason for these discrepancies was derived from the different patterns of recall organization. Moreover, they discussed that experts' high level exposure to both consistent and inconsistent situations, confidence level to cope with inconsistencies, attitude towards inconsistencies due to an opportunity of more ideological objections were the main underlying reasons for the preference of experts related to tendency towards inconsistent information.

Teaching and learning. Lee (2008) investigated the effect of corrective feedback, which was provided by collaboration and scaffolding of experts with novices, on forms of grammar usage in daily routine conversations. The experts were able to provide step-by-step scaffolding feedback to the students, who were learning a second language, during a computer supported chat session. The feedback called students' attention to use correct grammar resulted in corrections while they were in a conversion. 
The expert feedback helped students resolve the grammar problems and increase their confidence, which fostered a switch from other-regulated to self-regulated grammar and corrections.

Chiesi, Spilich, and Voss (1979) examined the effect of individual's knowledge level about baseball on the acquisition of new knowledge. They classified the study participants as processing high and low level knowledge. Along the four different experiments about the new game rules of baseball, they examined recognition, amount of information needed for recognition judgments, anticipation of outcomes, and recalling event sequences. Experts, in contrast to novices, showed superior performance on recognition, anticipation of outcomes, and recalling event sequences; they needed less information in order to judge.

Zahodnic (2009) investigated a potential use of the think-aloud process of experts as an instructional strategy for training of novices in respiratory therapy. $\mathrm{He}$ applied an experimental study, which did not yield any significant results in terms of decision-making performance. However, the groups which were instructed with thinkaloud protocol demonstrated higher proficiency, information gathering and decisionmaking efficiency, and they had fewer negative scores, errors of omission and commission. Moreover, the experiment group showed more aggressiveness to make decisions; however, they did not have any rationale for taking this decision action.

Tynjälä (1999) examined the possible impact of constructivist learning environments on the development of expertise during university studies. He argues that experts are critical and reflective thinkers about their thoughts and actions, such as problem solving, communication, corporation, and continuous learning skills; moreover, 
university teaching should promote the aforementioned skills. He further discusses that constructivist learning environments can provide these skills as outcomes. For this reason, he conducted an experiment that compared traditional and constructivist learning environments to what extent the expertise skills were acquired. The learning outcomes for each environment were analyzed in terms of subjective learning experiences, conception of learning, and traditional examination questions. For the first outcomes, both group students emphasized the learning of new knowledge; however, constructivist environment students also stated that they learned the ability to apply new knowledge. Both groups of students demonstrated changes in conceptions of learning, and there was no superseding group. For the third outcome, constructivist environment students demonstrated more outcomes of higher-level skills, e.g., classifications, comparisons, and generalizations.

Hsu (2006) investigated the effect of metaphors on the basic and integrative knowledge acquisition of both expert and novice computer programmers. He found that metaphors were shown consistency with the current understanding of experts; as a result, he indicated that metaphors strengthen the current knowledge of experts. For novices, he stated that novices learned integrative knowledge better with metaphors; furthermore, he summarized that metaphors worked more effectively for the learning of integration and relations of several concepts rather than understanding a single concept. In summary, his research demonstrates that metaphors foster improvement of mental models in both experts and novices. Experts reinforce their mental model and make minor modifications; novices can create relational knowledge and have ability to apply it. 
Haerem and Rau (2007) studied the influence of similar tasks that differed in complexity on the perception of task complexity and performance on individuals who are at expert, intermediate, and novice levels. They identified task complexity in three different levels: (a) deep, (b) surface, and (c) composite of deep and surface structured. They found that the perceptions of experts regarding task variability and analyzability were lower and higher respectively than intermediate individuals, who had also lower task variability and higher task analyzability perceptions than novices in the deep structured task. Moreover, they indicated that there was no difference among groups in terms of task variability and analyzability perceptions for the mixed structured task. However, the researchers hypothesized the contrast in results of the deep structured tasks in the surface structured task, and they were not confirmed. For performance results, experts outperformed intermediates and novices in the deep structured task; novices performed better than other groups in the surface structured tasks; in the mixed structured tasks, none of the groups surpass the performance of the other groups. In light of these results, Haerem and Rau (2007) identified the perceptions and performances of experts related to task changes when they develop from novice to expert. They also indicated an initial attempt to understand why individuals have different representations and performance in different tasks; thus leading to an extra attention on the classification of tasks types and individual expertise levels in all professional settings for designing talent management or knowledge management systems.

Clark (2008) indicates that experts not only acquire large amounts of knowledge of a domain but also organize this knowledge in a more complex way. Mental models 
are the memory structures that are stored in long-term memory, and they also provide a basis for expertise. She identified two types of mental models: (a) simple and (b) complex models. Simple mental models support cognitive operations, such as discriminations and generalizations; complex mental models meticulously focus on routine or novel problem solving. She further states that mental models that are necessary for building expertise must be supported with good learning environments where an active construction of new mental models is constantly supported. She indicates that there are two classes of methods to develop mental models. The first is implicit models including training techniques that do not need any behavioral action from learners, such as graphics, personalized writings, etc. The second is explicit models that utilize observable behavioral involvement of learners, such as practice exercises, argumentation, instructor questions, etc.

Lister, Simon, Thompson, Whalley, and Prasad (2006) examined differences between students (novices) and educators (experts) by considering the Structure of Observed Learning Outcomes (SOLO), which is an educational taxonomy, in computer programming reading exercises. The SOLO taxonomy contains four levels: (a) prestructural, (b) unistructural, (c) multistructural, and (d) relational. Prestructural level is the lower level outcomes, and it entails understanding misconceptions and preconception irrelevant to the actual problem that needs to be solved. The next level is unistructural in which students can understand the problem partially. Multistructural level includes understanding of all the parts of the problem; however, students are not aware of the relations between these parts. In the final level, relational, students understand all parts and relations of the problem coherently; thus, they can generate and apply 
solutions to it. The results indicated that educators were able to express relational responses; in contrast, students were able to convey multistructural responses. This study has an innovative nature since it analyzed the expert and novice differences in terms of outcomes dissimilar to the previous studies that have focused on cognitive processes or schemas.

Another study compared a young children's semantic knowledge of familiar and unfamiliar dinosaurs (Chi \& Koeske, 1983). It demonstrated that the visual map of familiar dinosaurs were formed more cohesively and interconnected; whereas, the more weakly and uniformly connected map was observed for the unfamiliar dinosaurs. This result emphasized the structure of knowledge and information acquired by a person at high knowledge level differs from the structure of low knowledge level.

Human Performance Technology. In Chapter 1 several studies, e.g., Perez and Emery (1995), Villachica, et al., (2001), and Witucki (2006). were presented regarding expertise in HPT. In this section, some additional studies focusing on expertise in instructional design are covered.

Rowland (1992) studied the differences between novice and expert instructional designers during the beginning phase of a project. He identified eight dimensions: (a) problem interpretation, (b) problem analysis, (c) problem representation, (d) solution generation, (e) the solution, (f) internal resources, (g) external resources, and (h) decision making. He indicated that expert instructional designers interpret a problem in an ill-defined form. They devoted a long time to analyze the problem and thought about solution ideas to limit the analysis. They established a casual network and more deep system understanding. They considered weak links to address attack points on casual 
networks. They thought about varieties of interventions. They employed their experiences as designers, and they had different templates and design principles as their toolbox. They did single reading. They made decisions based on multiple and global factors. On the contrary, the novice instructional designers perceived the problem in a well-defined form; in addition, they spent little time on analysis and moved to the solution generation. They represented the problems based on given information and surface features. They established strong links and identified knowledge deficiencies. They only focused on instructional interventions. They experienced the whole process as learners. They re-examined the external recourses constantly. They made decision based on single and local factors.

Le Maistre (1998) examined the performance of two instructional designers during the revision of instruction based on formative evaluation data. The first designer had 18 years of experiences and did not have an official advanced degree in instructional design; the second had five years' experience and a master's degree. The first designer was taught primarily by a mentor; the second took courses related to instructional design. Le Maistre (1998) compared these two instructional designers based on commonly accepted expert characteristics stemmed from the expertise literature. The characteristics are rich and well organized knowledge-base in the domain, deep problem representation, extensive front-end analysis, superior short-term memory, rapid and efficient search in problem space, and excellent self-monitoring skills. Consistent with previous expert studies, he found that the first designer possessed the aforementioned characteristics; whereas, the second designer focused on more surface and cosmetic revisions. She did not utilize instructional design 
principles, and she was not confident when she was making decisions. She usually lost track of the progress; moreover, she did not use most of the formative evaluation feedback.

Kirschner, Carr, van Merriënboer, and Sloep (2002) focused on the similarities and differences between the two groups of experts from different contexts. The first group was from academia, and the second was from business. They found that both of expert groups agreed on the importance of learner needs in design of instruction rather than on content structure of the learning domain. The main difference between the groups was the consideration of alternative solutions. The university group found this principle was particularly essential; however, the business group disagreed. Moreover, the university group was more prone to focus on the project plan and desired characteristics of instructional design blueprint; on the contrary, the business group was inclined to use a more client-oriented approach.

Perez, Fleming Johnson, and Emery (1995) examined the divergences of the problem solving process between expert and novice instructional designers during a design task. The analysis of think-aloud protocol established that there were certain differences between the design models utilized by each group due to implementing different problem solving strategies. Experts were more tied to use instructional design principles from numerous knowledge resources than novices. Consistent with previous literature, experts spent more time on front-end analysis processes than novices who started immediately thinking design strategies. Experts established more complex interconnectedness of the problem while novices had a few linkages. Interestingly, 
expert design models had a common characteristic that they were not in a linear form. Yet, they were iterative, cycling, and integrative.

The experts almost show common characteristics across diverse domains. Most of the differences are stemmed from the nature of subject matters. On the other hand, experts have differences among themselves since they are individuals, and individual differences matter in the environments where the human person is a key player. The next section represents the relationship of expertise with individual characteristics.

\section{The Relationship of Expertise with Individual Characteristics}

The relationship of expertise with individual differences are explained under intelligence, innate abilities, creativity, tacit knowledge, neuro-anatomic and neurophysiologic, age, personal traits, distinctive processing, and finally social perspective titles. Some characteristics are used to explain the nature of expertise; some evaluate the impact on expertise; another group of them are examined to observe the impact of expertise on the characteristics.

Intelligence. The most commonly known individual characteristic is intelligence. Horn and Masunaga (2006) makes a connection between theory of expertise and intelligence. They used Carroll's (1993) extended intelligence theory that explains intelligence as 40 primary abilities and eight-second-order factors of organization of these abilities; this theoretical framework provides the principal descriptive concepts of the theory. These eight second order abilities are as follows:

1. Acculturation knowledge (Gc) adheres to what extent an individuals' integration to knowledge and language of dominant culture is related. These abilities are explicit; therefore, they usually are taught in school curricula, but, 
indeed, they are outcomes of acculturation. In more basic terms, they are the abilities that are measured in IQ or scholastic assessment tests.

2. Fluid reasoning (Gf) contains reasoning abilities and support of these abilities. They are independent from acculturation. They are revealed in solving reasoning tasks, indentifying relationships, understanding implications, and summarizing inferences in problem situations. These skills entail immediate attention rather than long period of time tasks, such as writing an article.

3. Short-term apprehension and retrieval (SAR) is also known as short term memory or working memory. These skills are related to the individual's ability to hold information without rehearsal in the time duration of perception.

4. Fluency of retrieval from long-term storage (TSR) is also known as long term memory. These abilities cover an individual's ability to remember perceived information in associated manner after hours, months, or years. They are measures after a long period of time in contrast to SAR's immediate reaction.

5. Visual processing $(\mathrm{Gv})$ is regarding an individual's visual interpretation and identification of objects which are presented in different positions or angles.

6. Auditory processing $(\mathrm{Ga})$ includes recognition of sound patterns in either silent or distorted conditions and maintaining awareness of order and rhythm among sounds.

7. Processing speed (Gs) contains skills that are rapid scanning and comparing tasks in which usually people can get the accurate answer when they do it in ordinary speed. 
8. Quantitative knowledge $(\mathrm{Gq})$ is associated with mathematical knowledge and quantitative skills. For example, quantitative section of SAT, ACT or GRE exams measure these skills.

Horn and Masunaga (2006) identify theory of expertise based on the aforementioned extended intelligence theory. They contend that there are four classes, which are acculturation knowledge (Gc), fluency of retrieval from long-term storage (TSR), expert deductive reasoning (ExpDR), and expert working memory (ExpWM) of abilities play a critical role during the development of expertise. ExpDr is originally derived from fluid reasoning skills (Gf) but identified as different (Masunaga \& Horn, 2000). Hence, Gf is inductive; whereas, ExpDR, which needs to be accessed previously stored principles in the mind, is deductive. Like ExpDR, ExpWM is the different form of short term working memory (STWM). ExpWM differs from STWM in four ways (Ericsson, 1998): (1) the larger stored amount of information, (2) information less influenced from distractions and distortions, (3) flexible sequence and order of recall, and (4) the information stored in long-term memory and can be retrieved when it is required unexpectedly. These four classes of abilities are identified since they contain activities that are acculturation and learning in domain; therefore, they ensure becoming of an expert. However, classes of reasoning abilities (Gf), short-term apprehension, and cognitive processing speeds (Gs) are not influenced by the activities that an individual needs to become an expert (Horn \& Masunaga, 2006). Therefore, we cannot identify experts' performance based on them.

Innate abilities. The clear evidence stemmed from systematic laboratory demonstrated ". . . research on prodigies and savants provides no evidence for 
giftedness or innate talent but shows that exceptional abilities are acquired often under optimal environmental conditions." (Ericsson \& Charness, 1994, p. 729). However, a recent study by Grabner, Stern, and Neubauer (2007) demonstrated that expertise in chess was influenced in multi-dimensions of innate abilities, e.g., numerical intelligence, domain-specific performance motivation, and emotion expression control, and cannot be explained as experience in chess playing alone. There is an open room for the relationship of innate abilities and expertise regarding which one of them plays a more effectual role on exceptional performance. The future research will illuminate this debate.

Creativity. Weisberg (2006) investigated the necessity of expertise for creative thinking by analyzing several important historical figures in history, such as Mozart, The Beatles, Wright Brothers, and so forth. He specifically attempted to determine whether general or domain-specific expertise is sufficient to explain creativity. He discussed, based on analysis of each case, that general expertise knowledge could not be sufficient to understand creativity. He found that domain-specific expert knowledge somehow explained creativity of cases; however, the relationship between them was still complicated and inconsistent. He emphasized that the strong relationship between domain specific expertise and creativity was highly context dependent. For instance, in athletic sports and medical areas, the result of creativity supported by the domain expertise fostered quantitative changes. However, groundbreaking or radical creative works could not go beyond the old works quantitatively. They were considered as important since they are different from the older works. Weisberg's (2006) work on this relationship demonstrates that creativity and expertise are diverse across domains. In 
order to understand this relationship more clearly, these two entities must be explained under the domain investigated, and the next step should consider identifying this relationship.

Tacit knowledge. The study of expert performance and human development could mutually shed some insights on each other. Cianciolo, Matthew, Sternberg, and Wagner (2006) represented a linkage between the study of human development, which specifically focuses on practical intelligence and tacit knowledge, and the exploration of expertise. They identify practical intelligence as the ability to acquire tacit knowledge derived from everyday knowledge, and it needs to be applied in every day practical problems that have missing information and need a solution strategy. They further explain knowledge-acquisition components that are necessary for practical intelligence. There are threefold: (a) selective encoding, selection of information from the environment that is relevant to understanding the current situation and producing solutions, (b) selective combination, the integration of numerous pieces of selectively encoded information into a meaningful knowledge structure, and (c) selective comparison, the comparison of recently formed or adapted knowledge structure to the previously created one. Accurate execution of these cognitive processes ensures creation of tacit knowledge as well as practical intelligence and its behaviors. They also emphasize that multiple studies demonstrate the relationship between tacit knowledge and demonstration of expertise in different domains. Individuals with high tacit knowledge are more prone to perform at the expert-like level. For example, Wagner (1987) found a high association between psychology professors' tacit knowledge scores and the number of citations to their work in Social Citation Index (SSCI). 
Another study by Tan (1997) studied the relationship between tacit knowledge and financial auditors in business settings, and they found that there was a strong relationship between the level of tacit knowledge (i.e., low or high) and the level of expertise, exemplified by managers, senior, and staff. They summarized that the practical intelligence and tacit knowledge theory support the study of expertise by providing a better understanding of complex person-environment interaction that is critical in everyday life. It is interesting to mention that practical intelligence and tacit knowledge theory are stemmed from knowledge structures explained by the mental model theory. Cianciolo, et al.'s (2006) theoretical framework illuminates the impact of mental models on the development of expertise not only conceptually but also empirically.

Ford and Sterman (1998) portray an expert tacit knowledge elicitation method that utilizes formal modeling, which is a more detailed mathematical representation of a system than a conceptual model, and transformation of three description formats. In their method, there are three main phases: (a) positioning, (b) description, and (c) discussion. The positioning phase aims to identify a context and goals for the description process, and it includes three steps that are establishing context, focusing one relationship at a time, illustrating the method. In the description phase, experts are expected to describe relationships in their model in visual, verbal, textual, and graphical modes. The discussion phase tests, understands, and improves each description of different experts; it has examination and comparison of individual descriptions. They discussed several advantages of their model after implementation of it using product development project as example. These advantages were multiple formats of mental 
model representation, which might capture more amounts and quality of information, reduced cognitive processes by using step-by-step description of relationships in graphical format, using and providing complete documentation of the process, and more error-checking during the comparison stage.

Neuro-anatomic and neuro-physiologic. Hill and Schneider (2006) discussed the development of expertise from neuro-anatomical and neuro-physiological perspective. Their major focus is to explain the changes in human brain after acquisition and execution of skills. The human brain alters when there is a development and implementation of skills since it has a flexible and elastic structure that can manipulate the amount and the activity of areas as a result of training. There are two classes, the domain general controls and the domain specific representational areas, of the hundred specialized areas in the human brain. The domain specific representational areas include input, e.g., visual, audition, somatsensory, and gustatory, and output motor areas. There are structured quasi-hierarchically, which means the flow of information or pulses is reciprocal. There are numerous specialized processing regions. For example, visual processing incurs involvement of more than 30 different regions. The domain general controls are fewer than the representational areas, and they get involved in numerous tasks. Attentional control, process monitoring, decision making, conflict management, goal processing, tasks switching, emotional processing, episodic coding of association, smooth sequential processing, reinforcement and motor controls are the major example areas for the domain general controls. The existence of a single general domain control structure manages numerous numbers of domain specific representation regions; moreover, it has a prominent influence on understanding skills acquisition and 
performance. Hence, this single general network scaffolds novices to support new learning, retain active working memory, and alter cognitive processes that allow to vary the nature of the performance during completion of a task. When these tasks are consistent as in training programs, the domain general activity becomes automatic or omitted. Training especially makes changes on the domain specific representational areas, that is, when there is a change in cognitive processing at the end of the training, there is also a change in the nervous system. In summary, skilled performance are executed in the human brain by utilizing two different areas, which contain numerous complex sub-regions. The domain general control areas are responsible for facilitating skills acquisition; the specific representational areas are the locations where the skills acquisition happen as physiological neural changes. Training primarily influences in these representational areas; however, expertise cannot be succeeded without existence of the domain general control areas.

Age. The relationship between age and expertise is a controversial issue. For instance, Charness, et al. (1996) found that age had negative impact on chess players' chess skills in international tournaments. However, Day and Lord (1992) indicated that older experts demonstrated better performance than younger experts in terms of classifications of the organizational problems. According to Krampe and Charness (2006), since general cognitive abilities, which are usually measured by IQ tests, do not include skills, contextual factors, everyday competencies, real-life expertise, etc., the poor association between age and expertise at older ages cannot be completely accurate. For this reason, more ecological approaches to investigate this relationship emerged. Krampe and Charness (2006) states that the current research on expertise 
demonstrates that older adults can maintain their expertise if they keep their deliberate practice regarding the expertise domain at least up to age 70 s. They discussed that these results currently are promising since it can fulfill the high-demand of skilled workers on the market which occurs due to declining birth rate in developed societies.

Personal traits. Beier, Campbell, and Crook (2010) investigated the impact of cognitive ability and non-ability traits, such as personality, on learning derived from multiple-choice achievement tests and knowledge-structure accuracy. The personality factors identified as goal-orientation, i.e., mastery, performance-approach, and performance-avoid, conscientiousness, which stands for achievement motivation and dependability, and openness to experience. Knowledge-structure accuracy was measured by using Pathfinder networks in which the knowledge structures of students were compared to the expert referent model related to the topic of the course. The result of the study revealed that knowledge-structure accuracy, cognitive abilities, performance-avoid and mastery orientation had significant and positive impacts on learning. Knowledge structure accuracy was the second highest impact on learning after conscientiousness.

Distinctive processing. Rawson and Van Overschelde (2008) hypothesized that high knowledge individuals demonstrated superior memory over low knowledge level individuals since they had not only strong organizational processing but also more effective processing of differences among items under the context of the similarity. Their key point was stemmed from distinctive theory, which assumes that knowledge advances memory because of more useful distinctive processing of domain knowledge in addition to organizational processing. In other words, the positive impact of 
knowledge on memory occurs due to the effective combination of item-specific and organizational processing rather than only organizational processing. They believed that organizational processing dominated the explanation regarding expert superior performance; however, the role of distinctive processing has been neglected. To support their argument, they conducted several experiments in which individuals that had high and low level football knowledge were shown lists of words relevant to football and cooking and asked them to recall these words in the conditions that encouraged either distinctive or organizational processing. The experiment revealed that high knowledge individuals recalled more information in distinctive processing conditions than in organizational processing conditions. Moreover, high knowledge individuals outperformed and showed more improvement than low knowledge individuals. The study demonstrated that in addition to the organizational processing, the distinctive or item-specific processing might be another reason for expert exceptional performance in their knowledge domains.

Social perspective. The terms of expertise and experts are usually studied from personal characteristics and efforts in psychological perspective; however, the contextual factors mostly have been neglected. According to Mieg (2006), expertise is related to performance criteria within the context where the expertise is applied. He further expressed that expertise and experts always exist because of counter-distinct groups, such as non-experts, novices, or laypersons. This relative position creates not only information on the extent of knowledge and skills but also on social norms, such as power, prestige, or privileges. The social existence of expertise and experts is stemmed from understanding of expertise as forms of interaction that Jacoby and Gonzales 
(1991) depicted as basic features on expert and novice scientific discourse. Jacoby and Gonzales (1991) identified the dual and relative role of the experts and shifting of expertise in accordance with either domain or audience in the contexts. In light of their study, the expert-interaction is explained as process in which a person is introduced as an expert in front of an audience group since the person has knowledge to interpret a certain function, such as solving a commonly known problem. The process is circular. The interpretation of the certain functions provides information to the audiences. The constitution of expertise contains two main interactions: attribution of audiences to the person with high level knowledge and skills and interpretation of a function based on this person's knowledge. From the social perspective, expertise cannot exist only relying of an expert's exceptional interpretation of a function since it requires also an audience or a non-expert group's recognition. Moreover, it is a relative entity that is prominently relevant to context. This point of view brings the concepts of relative expertise that might produce exceptional performance in appropriate contexts even though expertise is not identified objectively. Mieg (2006) more broadly discussed that socialization is one of the important factors shaping the development of expertise since empirical factors, such as deliberate practice and long-term training, usually happens in certain contexts, and the development of expertise influences other people in these contexts. In the vein of Mieg's (2006) point, socialization might be considered as one of the impactful factors in expertise development.

Teams are small examples of social groups; therefore, the characteristics of expert team is another area for studying. Salas, Rosen, Burke, Goodwin, and Fiore (2006) summarized general characteristics, which are holding a shared mental model, 
practicing deliberately, and possessing both effective managerial and affective synergy, of expert teams. The shared mental model helps experts in team anticipate each other and decreases overt communication efforts. Expert teams deliberately practice by using a cycle of self-critiquing, learning, adaptation, optimization, and management of both resources and performance outcomes. Expert teams also have effective managerial and affective synergy since they have clear responsibilities, shared vision, built in trust, management of conflict, cooperation, and coordination.

In expertise literature, most of the time, experts' superior and exceptional performance, and the reasons of this performance are prioritized concerns for the scholars. However, similar to all other concepts, expertise has inherent weaknesses. The next section will explain these weaknesses in detail.

\section{Weaknesses of Expertise}

Experts have superior knowledge, skills, and abilities; however, this does not mean that they always do things or perform things perfectly. Of course, they have certain weaknesses. In this vein, Chi (2006) reviewed the ways experts excel and fail.

Table 1 illustrates a brief summary of her review.

Table 1. Ways experts excel and fail

\begin{tabular}{|c|c|}
\hline Ways experts excel & Ways experts fail \\
\hline - Generating the best solution & - Domain-limited superior performance \\
\hline - Detection and recognition & - Overly confident \\
\hline - Analyzing a problem qualitatively & $\begin{array}{l}\text { - Glossing over and cannot see } \\
\text { superficial features }\end{array}$ \\
\hline $\begin{array}{l}\text { - Accurate self-monitoring skills for } \\
\text { errors and their comprehension }\end{array}$ & $\begin{array}{l}\text { - Context-dependence within a domain } \\
\text { and relying on contextual cues }\end{array}$ \\
\hline - Choosing appropriate strategies & $\begin{array}{l}\text { - Inflexible and not open to different } \\
\text { rules }\end{array}$ \\
\hline - Being opportunistic & $\begin{array}{l}\text { - Inaccurate prediction, judgment, and } \\
\text { advise for novice performance }\end{array}$ \\
\hline $\begin{array}{l}\text { - Retrieving domain knowledge with a } \\
\text { minimal cognitive effort }\end{array}$ & - Bias and functional fixedness \\
\hline
\end{tabular}


There have been several studies confirming these weaknesses. Brockmole, Hambrick, Windisch, and Henderson (2008) compared the chess expert and novices' understanding and mental representation of association between a search target and its contextual surroundings in two experimental visual tasks to explain learning and retrieval of visual information. In the first experiment, which applied actual game play and search benefits for repeated boards, they found that experts performed four times better than novices in finding the target. In the second experiment, they randomly generated the boards, which include less meaningfully positioned chess pieces to the participants; experts performed better than novices; however, their performance were halved. They discussed that an individual's ability to make the meaningful connections of a target object with other contextual components was strictly relevant to the individual's ability to distinguish the target and the quality and the nature of visual inputs from these contextual components. In addition to its purpose, this study revealed a weakness of experts, which is the domain-specific nature of expert knowledge. When they come across with unfamiliar contexts or patterns, their performance is hindered. In these conditions, experts need to exceed their usual boundaries and generate a new way of thinking, which is also called adaptive expertise (Clark, 2008).

Waters and Gobet's (2008) study applied a new experimental approach in which chess pieces were located in intersections of squares rather than centers for examining expertise in chess domain. After they predicted the potential impact on mental imagery and chunking via CHREST simulation, a model of learning and expertise that accounts for data on perception, learning, and memory in diverse domains, they applied the 
experimental tasks to human subjects to control CHREST prediction accuracy. First, the simulation predicted that intersection positions would be recalled worse than standard positions, and human subjects confirmed that. Second, it predicted that the skill level, which are new players and grandmasters, in recalling game positions were greater than randomized positions; the results of human subjects also were consistent with the second prediction. Third, time parameters prediction was the same consequences similar to the first two predictions and the human subject side results. Overall results demonstrated that chess pieces should be placed at the center of the squares to activate and take advantage of mental imagery and chunks. Moreover, the study demonstrates that experts demonstrated their skills in the more familiar condition outstandingly; however, uncommon situations hinder their usual exceptional performance.

Castel, McCabe, Roediger, and Heitman (2007) examined the possible intrusion effect of the superior knowledge organization and retrieval abilities of experts. They chose individuals with high and low level knowledge of American football. They showed the participants two sets of words. The first set had animal names used in the current league; the second set had names of body parts. They used two different sets since they tested both domain specific and general knowledge. In the experiments, they showed about ten names; however, during the assessment test, they asked additional three names to observe intrusion impact of them on recalling. The study results indicated that high knowledge level participants recalled more names of both animals and body parts, but they also made more mistakes by recalling names not showed in the tasks than low knowledge level participants. They discussed that experts take 
advantage of their superior knowledge organization and recalling skills; nevertheless, they also might retrieve unnecessary information so this hinders efficiency of their performance.

Knowledge elicitation techniques are commonly utilized for understanding both strengths and weaknesses of experts in terms of organizing knowledge. Cooke (1994) presented a good overview of elicitation techniques. One of the techniques that she explained is Pathfinder scaling, which will be utilized in the present proposed study. The next section of this literature review presents several studies that examined or used this technique.

\section{Pathfinder Scaling: An Innovative Technique to Elicit Knowledge Structure}

Since Pathfinder is a relatively new technique, the validity of the technique is necessary. There are two types of studies that are covered in this section. The first, validity studies, cover example studies that showed that Pathfinder technique and its components work as desired in different settings. The second title elucidates the studies that utilized the technique as a tool.

Validity studies. There have been four types of validity studies related to Pathfinder scaling. The first has been related predictive validity for the outcomes of teaching or training programs. The second has been the comparison of the Pathfinder technique with other techniques. The third has been assessment of expert referent model, which is a component of Pathfinder scaling. The fifth has been analysis of specific sub-relationship within the whole knowledge structures.

Dorsey, Campbell, Foster, and Miles (1999) assessed the relationship of knowledge structures to experience and post-training performance, and the validity of 
two knowledge structure measuring techniques, Pathfinder networks and concept mapping. In their study, they did not find any significant effect of knowledge structures on post-training performance; however, they discussed the results that short training programs were not sufficient to make drastic changes, which facilitated performance, on knowledge structures. A long period and an extensive amount of training might develop knowledge structures that assist acquisition of skills and learning in novel circumstances.

Goldsmith, Johnson, and Acton (1991) evaluated the validity of the Pathfinder method for students' cognitive representations of classroom learning. They applied the structural approach, which aimed to reveal the structure of an individual's domain knowledge, in three steps: (a) knowledge elicitation, (b) knowledge representation, and (c) evaluation of an individual's knowledge representation. As a result of their study, they indicated that Pathfinder technique had an important potential as an individual assessment tool for gauging the classroom learning of students regarding some idealized level of expertise. They further discussed that the relatedness rating, which is the data collection process of Pathfinder technique, minimized the direct conscious judgment of relations as well as the elicitation of the knowledge structure. They suggested that in order to ensure validity of Pathfinder technique, a small set of concepts that are related to the assessed performance might be used.

In structural knowledge assessment process, expert referents have a critical role during the evaluation of the knowledge structures of individuals. Expert referent approach assumes that there is an ideal structure of domain knowledge, and cognitive structures or mental models advances to this ideal model as a result of more 
experiences in the domain (Acton, Johnson, \& Goldsmith, 1994). Even though the approach depicts the ideal structure, there is not any ideal group of people who can be utilized to create these referent structures. For this reason, Acton, et al. (1994) conducted a study to compare the validity of four different types of expert referent structures: (a) individual instructors, (b) non-instructor experts, (c) averaged experts, and (d) averaged good students. The results of the study indicated that averaged expert referents were relatively higher and better predictive and reliable referents than the other referents.

Kahler (2003) compared two knowledge acquisition methods, which were rating and backward thinking, for the elicitation of procedural mental models. She utilized Pathfinder network analysis for rating methods, and she analyzed the relationship of similarity and coherence indexes with the project scores of students in a computer programming course. She found that the similarity indexes, which were generated by the comparisons of students' Pathfinder network results with the instructor's network, had a predictive validity on students' project performances. She further suggested that rating methods should be placed as a task during instruction to improve mental models of students.

An interesting study was conducted by Schlomske and Pirnay-Dummer (2009) on the relationship of acquisition of expertise and learning dependent model changes of students. They found that there were remarkable model changes while students were progressing from novices to experts, who were identified as advanced learners in the study. They argued that reference models, which were advanced learners' models, were suitable to predict the acquisition of expertise. 
Schuelke, Day, McEntire, Boatman, Boatman, Kowollik, and Wang (2009) studied the criterion validity of the knowledge structure parameters derived from KNOT - Pathfinder algorithm software on skill acquisition and transfer. Coherence, which explicates the internal consistency of a knowledge structure, closeness, which explains the similarity of a knowledge structure with a referent structure, and correlation, which elucidates the degree of the relationship between a matrix of relatedness ratings and a matrix of referent structure, were the parameters investigated. They also examined the number of links in a knowledge structure. They called correlation and closeness as accuracy-based indices since they were stemmed from the comparison with a referent structure. The results of the study indicated that three of the indices had significant validity on skill-based performance; nevertheless, accuracy indices were better than coherence. On the other hand, the number of the links did not yield any significant results. The authors further indicated that when the combination of accuracy and coherence indices was used, they would better explain skill-based performance than these indices as stand-alone indices.

Lau and Yuen (2009) investigated the predictive validity of Pathfinder scaling algorithm on the secondary schools students' computer programming performance by using expert-referent based and expert-referent free approaches. They found that the validity of these two approaches were almost the same. For expert-referent based approach, they utilized three different measures that are (a) correlation of raw proximities, (b) set-theoretic measure, which was the averaged ratio of number of links in common to the number of the nodes in either network for each node of the network, and (c) graph-theoretic measure, which was the correlation of the distances between 
the nodes in two networks. They indicated that the first measure had higher predictive validity than the other two measures, which had the similar predictive validity. They compared expert-referent free approach to the previous studies utilizing the same or similar approaches and indicated strong validity; nevertheless, they did not find a strong predictive validity in this study.

In previous knowledge structure studies, researchers usually evaluated the effect of total structures on knowledge or skill acquisitions; nevertheless, Trumpower, Sharara, and Goldsmith (2010) followed a different approach, and they examined the impact of the specific links in an individual's knowledge structure on his/her strengths and weaknesses. They developed two problem-solving tasks in computer programming. The first pointer-type problem; the second was go-to-type problem. They explained that one needs to know the concepts pointer, assign, position, and increment to solve pointertype problems, and go-to-type problems can be solved when the concepts if-then, go-to, and step concepts are known. They further stated that in previously developed expert referent structures, assign, position, and increment concepts were related to pointer concept; they hypothesized that an individual who has this conceptual relationship in his/her knowledge structure is more prone to solve pointer-type problems. They explained that if-then and step concepts were linked to go-to concept, and one who has these two links are more expected to solve go-to-type problems. The result of the study indicated that individuals who had position sub-set links performed better than those who were not in the pointer-type problem types. The same result was revealed for the go-to sub-set links in go-to types problem solving. The authors concluded that 
Pathfinder networks not only as a whole but also as specific sub-sets are useful tools for classroom and formative evaluation.

Studies utilized pathfinder as a tool. Clariana and Wallace (2007) examined validity of analysis of lexical aggregate (ALA) software, which analyzes an essay, transforms it into a relational data for Pathfinder analysis, and scores it, by comparing it with human rater scores. They found that the innovative technique was somehow valid. They also examined high and low performing students' essay. They indicated that high performer students demonstrated similar knowledge structures to expert structures. They also summarized that Pathfinder analysis was an appropriate technique to elicit expert and novice similarities. In addition to this, Clariana, Wallace, and Godshalk's (2009) study, which examined the effect of pronouns on computer-based essay analysis tools, discussed the approach to utilize expert referent structures in empirical studies that conversion of expert essay to an expert referent created an over-specification problem. They further suggested that a particular design used by an expert referent might reduce errors and false results rather than creation of the expert referent from any components of the research. Their suggestion is a critical consideration for future studies since creating expert referent structures from a selected small part of research might show the same error characteristics that would hinder clear interpretations of results. Expert referent models should be studied independently from participants of a research study.

McGaghie, McCrimmon, Mitchell, Thompson, and Ravitch (2000) examined students' concept learning in pulmonary physiology by evaluating the coherence, student-instructor similarity, the correlation of similarity with final test scores of the 
concepts maps, which were derived from Pathfinder network scaling algorithm. The results indicated that the instructors and students concept maps were coherent, and students' concepts maps were gradually approaching the instructors' concept maps during instruction. However, the similarity of students' and instructors' concept maps did not yield any significant relationship.

Day, Arthur, and Gettman (2001) investigated to what extent knowledge structures explain skill acquisition, retention, and transfer. They applied their study while participants are trained about a complex video game; they measured the skills acquisition at the end of the training and the skill retention and transfer after four nonpracticed days. The results of the study indicated that the similarities of trainees' knowledge structures with expert referent structure were associated with skill acquisition; moreover, it was projecting skill retention and transfer. The method to elicit expert referent structure affected the magnitude of these relations. Another important result was that there was a mediation effect of knowledge structures between cognitive abilities and skill-based performance.

DiCerbo (2007) utilized Pathfinder algorithm to compare the conceptual understanding of both students and instructors at the end of a computer networking training program. He indicated that the instructor's concept map included more theoretical and relational information; whereas, the concept maps of the students had more computer networking equipment information. In this vein, he concluded that instructor's concepts maps would be useful to define a relatively new field, and the differences between the students' and instructors' concept maps would be used to 
improve the current instruction. This study is an addition to other Pathfinder studies since it emphasizes the diagnostic nature of the scaling technique.

Rose, Rose, and McKay (2007) investigated the consequences of experience, instruction, and decision-making aid on declarative and procedural knowledge during the acquisition of expert-like knowledge structures. They indicated that knowledge structures generated with Pathfinder algorithms were effective means to assess the impact of training and decision-making aids on the development of expertise. They discussed that knowledge structures might be complementary tools in domains where traditional methods for assessment are not adequate and practical. Moreover, they found that properly designed decision aids conveyed novice decision makers for expertlike knowledge structures.

Rowe, Schvaneveldt, and Bennett (2007) investigated changes in combat pilots' knowledge structures during a networked training simulator session. Pathfinder scaling technique was used. The study examined the coherence and similarity to expert referent of the pilots' knowledge structures over the time of the training. The results indicated that the coherence scores were increased after the simulation training; moreover, the correlation between the coherence scores and the expert with the highest score was increased; however, there was no significant relationship when the average expert scores were used.

Lau and Yuen (2010) investigated associative effect of gender, learning styles, and mental models on the learning of sorting computer programming algorithms. They used The Gregorc Style Delineator, which classifies learning styles as concrete and abstract, and Pathfinder scaling for eliciting mental models. They found that females 
had more similar mental models than males; moreover, the results indicated that concrete learners had more similar mental models with expert mental models than abstract learners. They further discussed that gender and learning styles can be utilized for relating mental models; therefore, this promotes group-based diagnosis and remedies for instructional problems as well as conceptual changes.

Rentsch, Mot, and Abbe (2009) conducted a study to identify a schema for cultural understanding for Army leaders who got involved in multinational alliances. They would like to use this schema for further training of soldiers who need to work in unfamiliar cultures. Three cohorts of soldiers, who had previous experiences with different cultures, were used for the study. The first two groups were asked to identify cultural schema content statements; the third group was asked to sort these statements and complete as structural rating. They indicated that sixteen core statements were indentified, and the concepts of religion, values and beliefs, customs and traditions were the core attributes of the cultural understanding. This extensive study is remarkable for two points. The first was the combined implications, which are defining a phenomenon and designing training programs in accordance with expertise research. The second was utilization of Pathfinder scaling technique to reveal cognitive schema of individuals.

\section{Summary}

Experts have similar characteristics across different fields. On the other hand, they have also individual differences providing rich diversity. They are not only exceptional performers but also collections of a prominent source for either a better understanding or a direction for training and education of a domain. Therefore, understanding the experts from all possible dimensions will support both the theoretical 
and practical body of knowledge. Knowledge organization skills of experts are one of these dimensions which need attention since it is the vital piece of expert performance. Mental model theory clearly identifies the knowledge organization, its characteristics, and the approaches for researching it, and, expertise research consistently uses mental model theory. Unsurprisingly, the research trends in HPT have emphasized the necessity of expertise research via investigating the mental models of HPT experts. This is the main motivation and rationale of ideas and intentions to propose the present study. The next section will explain the methodology of the study. 


\section{CHAPTER 3}

\section{METHODOLOGY}

\section{Introduction}

The research design and procedures that were used in this study are explained in this chapter. The chapter has six main parts: participants, research design overview, instrumentation, data collection procedures, data analysis, and potential limitations.

The primary purpose of this study was to reveal performance improvement practitioner expert and novice mental models and identify differences and similarities between these models. The secondary purpose was to analyze the potential impact of the professional profile characteristics of performance improvement practitioners on their mental model of expertise. The following research questions were addressed:

1. What is the common mental model of expert performance improvement practitioners?

2. What is the common mental model of novice performance improvement practitioners?

3. What, if any, are the similarities and differences between the common mental models of experts and novices?

4. What are the professional profile characteristics of performance improvement practitioners?

5. To what extent are the professional profile characteristics of performance improvement practitioners associated with their mental models of expertise derived from Pathfinder? 


\section{Participants}

There were two participant groups for this study. The first group was experts who participated in the first phase of the study, and they were identified by using purposive sampling technique. Purposive sampling uses researcher's judgment to gather specific information about a topic since the population's personal knowledge is assumed representative (Wallen \& Fraenkel, 2001). The main disadvantage of purposive sampling is that the researcher's selection judgment may be inaccurate. To eliminate this obstacle, members of the first group will be selected based on four previously used criteria and one additional criterion selected by the researcher. Three of previously used criteria were adapted from Huglin, et al. (2007), and the last one was derived from the empirically proven principle that ten years of deliberate practice experience are necessary to become an expert in a domain (Ericsson \& Charness, 1994; Ericsson, et al., 1993). The five criteria for selecting experts are noted as follows:

1. those who had written extensively in the field of performance improvement,

2. those who have been active in the professional organizations, such as International Society for Performance Improvement (ISPI), The American Society for Training and Development (ASTD), or Association for Educational Communication and Technology's (AECT) Training and Performance Improvement track,

3. those who were recommended by persons identified via the first two criteria, and 
4. those who have had experience in performance improvement field for ten or more years.

5. those who have completed numerous HPT related projects.

57 experts were chosen for the first phase of the study. In the first round of contacting the experts, 57 experts were e-mailed. Four experts were recommended by the experts who were already in the list and had responded to the questionnaire. Three of these recommended experts were already in the initial list. One expert was not in the list so he was included in the list as well. With addition of the last expert, the total number of experts contacted reached to 58.23 experts $(40 \%)$ completed the questionnaire in a two-week period in the first round. In the second round of contacting the experts, the same 23 experts responded in the first rounds were e-mailed again. However, they were asked to complete the Professional Profile Characteristics and the Concept-Pairs Comparison questionnaires. 16 experts (70\%) out of 23 completed both of the questionnaires, and two experts (9\%) did not complete any of the questionnaires.

The second group was professional performance improvement practitioners currently active in the field. For this group, a convenience sampling strategy was used with an online survey tool that reached participants who were easily available for the study (Wallen \& Fraenkel, 2001). The members of three leading professional organizations (International Society for Performance Improvement, The American Society for Training and Development, and Association of Educational Communication and Technology) were invited to participate in the present study as the professionals. The research policies of these organizations are as follows (See Appendix D for support letters): 
- ISPI policy allows students to place a call for participants in ISPI's online newsletter www.performancexpress.org. This method enables them to collect responses from members and non-members of ISPI. In addition, ISPI also provides limited permission to target Certified Performance Technologist population by providing information and a link to the online survey in ISPI's monthly CPT-exclusive email newsletter.

- ASTD policy states that scholars can post messages in the official discussion forums and social networks of ASTD for their invitation to their research (See Appendix D). Moreover, ASTD encourages using their member's directory to obtain contact information if the researchers are members of the organization. ASTD does not have any other specific policies regarding research studies.

- AECT policy states that a scholar needs to submit a letter of application that must provide information about the research and the primary investigator. The executive committee and the Training and Performance Division leadership will review and decide to provide their member's contact information. Once they have approved, the primary investigator has to provide HIC approval and copies of the instruments.

In the light of above policies, the professionals in performance improvement field were contacted. The professionals were asked to complete an online survey. The researcher also utilized social networking sites, e.g., Facebook and Linked-in, and personal contact, e.g., personal address book and previously obtained business cards. 
When the personal contacts were used, an invitation e-mail was directly sent out to the professionals' e-mail addresses.

335 practitioners initially started the online survey; $272(81.2 \%)$ practitioners completed the Professional Profile Characteristics questionnaire, and 242 (72.2\%) practitioners completed both the Professional Profile Characteristics and the ConceptPairs Comparison questionnaires. 93 (27.8\%) participants had never completed any of the questionnaires.

Finally, in order to prevent any confusion related to the participants of the study, the experts were used for the first group participants, and the practitioners were used for the second group of participants in the study. The experts were selected based on the five criteria and contacted twice for the first phase of the study; the practitioners were contacted once via three professional organizations for the second phase of the study. 23 experts provided the concept ranking results, and 16 experts of these 23 additionally provided the data for generating the common mental model of experts - the expert referent model. The next section will explain research design overview in which all phases of the study are explained as well.

\section{Research Design Overview}

Phase 1: Identification of concepts and expert referent model. The first phase of the study identified the 11 critical concepts in the performance improvement field and established as an expert referent mental model (the common expert model) that was used in the second phase of the study. The experts were contacted twice. In the first round, they provided rankings for the 30 concepts presented; in the second 
round, they provided professional profile characteristics and their concept-pair ratings. Survey technique was used to obtain feasible and efficient data.

In the first round of contacting the experts, the Online Ranking Questionnaire including the list of 30 concepts were sent out. These 30 terms were identified by the researcher after the review of numerous models in the field, e.g., ISPI, 2012; Faure, Rosenzweig, Van Tiem, 2010; Silber \& Foshay, 2010; Januszewski \& Molenda, 2007; Phillips and Phillips, 2007; Rummler, 2007; Addison \& Haig, 2006; Barab, Warren, del Valle \& Fang, 2006; Pershing, 2006; Van Tiem, Moseley \& Dessinger, 2012; Kaufman, Oakley-Browne, Watkins \& Leigh, 2003; Molenda \& Pershing, 2004, Atkinson \& Chalmers, 1999; Burton \& Merrill, 1991, Kaufman \& English, 1979; Gilbert, 1978). Sufficient resources, talent management, and team development were included in the list after a brief discussion with the dissertation advisor. The 30 concepts were listed in Table 2.

Table 2. 30 concepts selected for the Online Ranking questionnaire

\begin{tabular}{|l|l|}
\hline Added-value & Intervention Implementation \\
\hline Appreciative Inquiry & Intervention Selection, Design \& Development \\
\hline Cause Analysis & Knowledge \& Skills \\
\hline Communities of Professional Practice & Needs Assessment \\
\hline Cost-Effectiveness & Performance Analysis \\
\hline Critical Business Issue(s) & Return on Investment \\
\hline Establishing Partnership with Client & Strategic Planning \\
\hline Ethical Dimensions & Strategic Thinking \\
\hline Measurement \& Evaluation & Sufficient Resources \\
\hline Financial Management & Systematic Approach \\
\hline Focus on Results & Systems View \\
\hline Gap Analysis & Talent Management \\
\hline Incentives \& Motives & Team Development \\
\hline Individual Capacity & Training \\
\hline Instructional Design & Work, Worker \& Workplace \\
\hline
\end{tabular}


The experts ranked 30 concepts in accordance with their criticality to the general understanding of the field. The experts also responded to an open-ended additional question. The main purpose for this question was to obtain other concepts that might be neglected by the researcher. At the end of the first round, a list of 11 critical concepts was obtained.

In the second round of contacting the experts, Structural Assessment of Knowledge technique including (a) knowledge elicitation, (b) knowledge representation, and (c) knowledge evaluation, was utilized (Trumpower, Sharara, Goldsmith, 2010). In knowledge elicitation phase, the Professional Profile Characteristics and the ConceptPair Comparison online questionnaires were sent out. In the Concept-Pair Comparison questionnaire, the number of pairs as well as items in the questionnaire were 55 that was calculated by the formula $\left(n^{2}-n\right) / 2$, where $n$ is equal to the total number of concepts. The experts rated the relationships of each pair using the relatedness scale from one to seven. The scales one, four and seven will stand for very low, medium, and very high relationships respectively. The middle point scale four was identified to help participants make clear and distinct selections. The Concept-Pair Comparison questionnaire provided the ratings data of the concept pairs, that is, raw proximity data. In the knowledge representation phase, the proximity data from each expert were transformed into a group data by calculating averages, and the grouped data were analyzed by using Knowledge Network Organization Tools (KNOT). It is specifically developed software that reduces raw proximity data into PathFinder Network (PFNet) representation, which is a graphical representation containing concepts as nodes and relationships as links. The deliverable of the second round was a common mental 
model graphic that was utilized as an expert referent model in the second phase primary research procedures of the proposed study. The knowledge representation phase continued and the knowledge evaluation was initiated in the second phase of the study.

There are two parameters $q$ and Minkowski's $r$ to reduce proximity data into a PFNet. Minkowski's $r$ ranges from 1 to $\infty$ and $q$ ranges from 2 to $n-1$, where $n$ is equal to the total number of concepts or terms. The $q$ parameter is used for calculating weight between concepts or terms; the Minkowski's $r$ parameter is used to set the depth and length of the path. A path created by using these parameters is identified as PFNet (q, r). Changing these parameters will result in changes in the PFNet. The larger the meaningful values of the parameters, the fewer links in the PFNet there are as well as less complex graphical models. The smaller values of the parameters there are, the more detailed and complex links in the path there are. Using the largest values for the parameters is recommended for ordinal data type (Schvaneveldt, n.d.). In the proposed study, since a relatedness scale will be used, data will be ordinal. Moreover, when individual proximity data are used, $q$ should be $n-1$, and when average proximity data are used, q should be 2 (Rowe, et al., 2007; Schvaneveldt, 1990).

The Pathfinder analysis technique will be used since PFNets have the following advantages (Dearholt \& Schvaneveldt, 1990):

1. The capability of directly modeling asymmetrical relationships in which entities are not related to each other with the same power,

2. A more accurate representation of local data relationships and a complementary alternative nature, 
3. No hierarchical constraints,

4. New paradigm in studying models of classification,

5. A more quantitative paradigm than the networks derived from qualitative or designed intuitively.

The first two of the advantages are superior to multidimensional scaling; the third one supersedes cluster analysis; the last two surpass techniques, such as interviewing, think-aloud, text comprehension, etc. A major disadvantage of PFNets is that the similarity perceptions source of the PFNets data cannot represent features of which similarity judgments are made; however, the estimation of similarities are considered. On the other hand, the nature of PFNets provides an alternative way in which network structures can be created more objectively based on data rather than using researchers' intuitive preferences or beliefs.

Phase 2: Primary research procedures. In Phase 2, the practitioners were reached via three professional organizations (ISPI, ASTD, and AECT Training \& Performance Division). The practitioners were asked to complete the Professional Profile Characteristics and the Concept-Pair Comparison questionnaires like the experts completed at the second round. The practitioners applied the same steps that the experts applied in the second round of the first phase. Thus, each practitioner provided proximity data. 33 novices were selected from 242 practitioners based on the expert selection criteria explained in the method section since those 33 practitioners did not have neither more than ten-years experience in the field, nor extensive publications, and were not active in professional organizations. Moreover, they had not completed numerous projects, and they were not suggested by any of the experts. The proximity 
data of these 33 novices were analyzed by KNOT utilizing Pathfinder Network Analysis. 33 novices' ratings to each 55 concept-pair were averaged, and then these averaged scores were used to derive a common mental model of novices.

Relatedness, coherence, and similarity scores can be generated by KNOT software utilizing the Pathfinder Network Analysis technique to quantify mental models of individuals. These three measures are explained as follows (Villachica, 1999; Goldsmith \& Davenport, 1990; Goldsmith \& Johnson, 1990):

- Relatedness is a Pearson-Product Moment correlation value between sets of concept ratings. It theoretically ranges from minus one to plus one. In the present study, the average rating scores of 16 experts, used to create the expert referent model (the common expert model), for each 55 concept-pair comparison were correlated one by one to 242 practitioners' ratings for the same 55 concept-pairs set. The same measure was also calculated for each of 16 experts separately.

- Coherence is a Pearson-Product Moment correlation indicating internal consistency of ratings with an individual's or group's sets of concept ratings. It theoretically ranges from minus one to plus one. In the present study, each participant's and expert's coherence scores were calculated by using KNOT software. The same measure was also calculated for each of 16 experts separately.

- Similarity is the number of links in common divided by the total number of unique links in the two networks. Two identical networks will yield a similarity of 1 and two networks that share no links will yield similarity of 0 . 
In the present study, 242 participants Pathfinder models with 11 concepts were compared to 16 experts' model to obtain the similarity scores. The same measure was also calculated for each of 16 experts separately.

These three measures were used to quantify the mental models of the practitioners, called the mental model of expertise. Schuelke, et al. (2009) indicated that when the combination of accuracy (i.e., relatedness and similarity) and coherence indices were used, they would better explain skill-based performance than these indices as stand-alone indices. For this reason, the mental model of expertise was computed by summing values of relatedness, coherence, and similarity measures. Since this measure distinguishes the expert mental model from the novice mental model, it needs to have concurrent validity (Trochim, 2006). To ensure this, an independent sample ttest was performed to check whether the 16 experts' scores were significantly higher than the 33 novices' scores. The result of the test demonstrated that the experts' scores were higher than the novices' scores, $t(1,41)=4.533, p<.01$. Furthermore, the relatedness, coherence, and similarity measures were also examined. The relatedness scores were significantly different in favor of the experts, $t(1,43)=5.627, p<.01$; the coherence scores were significantly different in favor of the experts, $t(1,41)=2.059, p<$ .05 ; the relatedness scores were significantly different in favor of the experts, $t(1,47)=$ $4.804, p<.01$. As a result, the mental model of expertise had the concurrent validity for distinguishing the experts and novices.

\section{Instrumentation}

The first phase of the study utilized the Online Ranking Questionnaire (See Appendix E) at the first round of contacting experts. The experts ranked 10 critical 
concepts out of 30 in the questionnaire. A table of 30 concepts, which were identified after reviewing numerous models in the field, was provided. Then, the experts were asked to choose drop-down answers to identify the rank of a concept that are critical to the general understanding of the field. The experts were also asked to fill additional comments and thought in an open-ended question. Furthermore, the names, surnames, e-mail addresses of other experts that might be interested in participating in the study were requested from the experts. The additional comments and other experts' contact information sections were voluntary; the ranking section was mandatory to complete. The Online Ranking Questionnaire was completed by 23 experts out of 58.

At the second round of contacting the experts, the Professional Profile Characteristics (See Appendix G) and the Concept-Pairs Comparison (See Appendix F) questionnaires were sent out. They were provided in one web-site. After completing the Professional Profile Characteristics, the experts were able to access the Concept-Pairs Comparison questionnaire. The content of Professional Profile Characteristics were identified by utilizing previous existing studies, e.g., number of publications in the field from Villachica, et al., (2001), ten-year of experience from Ericsson and Charness, (1994), and the activities required to obtain and continue Certified Performance Technologist certification processes, e.g., the number of projects completed, the volunteering activities, board membership, participation to conference, etc. The main purpose of the questionnaire was to obtain information about the previous professional activities that the experts completed to date. The Professional Profile Characteristics questionnaire contained also age, gender, and job title demographic information. Both 
of these questionnaire were completed by 16 experts out of 23 experts who were completed the Online Ranking Questionnaire.

The Concept-Pairs Comparison questionnaire included 55 pairs of concept to rate the relatedness of two concepts in each pair. 55 pairs were created by using 11 concepts identified after analyzing the results of the Online Ranking questionnaire in the first round of the first phase. A detailed direction was provided at the top of the instrument. A relatedness scale was provided with ranges from one (low relationship) to seven (high relationship); there was also a value of five (moderate relationship). The experts were asked to read all pairs and choose the most appropriate relatedness value. The main purpose of this questionnaire was to obtain proximity data from each expert for KNOT software utilizing Pathfinder analysis.

In the second phase, the practitioners also completed the Professional Profile Characteristics and the Concept-Pairs Comparison questionnaires as the experts did in the second round of the first phase. The Concept-Pairs Comparison questionnaire was applied as identical; however, the Professional Profile Characteristics questionnaire included one addition question, which was asking the practitioners for providing their email addresses if they wanted to be considered for $\$ 25$ Amazon.com gift card incentive. The researcher used this information to contact the selected practitioners to get their mailing address for sending the gift cards.

Content validity of the instruments. Since the nature of the content is critical to this study's instrument, content validity, which is based on the evidence ". . . relies on the judgments of people who are presumed to be knowledgeable about the variable being observed." (Wallen \& Fraenkel, 2001, p. 89). Content validity presumes that “. . . 
experts are aware of nuances in the construct that may be rare or elusive of which the layperson may not be aware." (Rymarchyk, 1996, para. 9). The first phase of this study, specifically, was conducted in order to obtain valid concepts as well as the ConceptPairs Comparison questionnaire. Creswell (2012) explains validity evidence based on test content can be analyzed by examining logical or empirical evidences from the existing documents, publications, books, and so on so forth in addition to the experts' judgment. The Online Ranking and the Professional Profile Characteristics questionnaires were developed on the basis of available books, empirical publications, and standards previously identified practices expected from performance improvement experts and practitioners in the field.

\section{Data Collection Procedures}

In the first phase of the study, a list of 57 experts including their full name and email addresses where identified based on the five criteria aforementioned. An invitation e-mail cover letter (See Appendix C) that contains a link to the Online Ranking questionnaire was delivered. When they clicked the link, the information sheet approved by Wayne State University Human Investigation Committee informed the experts about the research study. The experts had to confirm that they agreed to participate in this study. Then, they saw the Online Ranking Questionnaire. The experts were given two weeks to complete the questionnaire. At the first invitation, 15 experts responded. The same invitation e-mail was sent out after one week to the experts who had not yet responded. Four experts were recommended by the experts who had already responded to the first invitation. Three of them were already in the initial list, and only one expert was added the list of the second invitation. At the second invitation, 8 more 
experts responded. The first round of data collection started on June 14th, 2011 and ended on June 26, 2011. After June 26th, the questionnaires were closed in Survey Monkey; therefore, there were not accessible any longer. The total 23 experts completed the Online Ranking Questionnaire. In the second round of contacting experts, an invitation cover letter e-mail (See Appendix C) sent out to those 23 experts. This invitation e-mail reminded the expert about the Online Ranking Questionnaire that they filled before and asked for their participation to the second round. Since they had already agreed to participate, they were not provided with an information sheet. Like the first round, the same invitation e-mail was sent out to the experts who had not responded yet after two weeks. 10 experts responded in the first invitation; 6 more experts responded in the second e-mail invitation. The second round started on July 27th, 2011 and ended August 5th, 2011. After August 5th, the all survey page was closed in Survey Monkey; it was not accessible any longer.

In the second phase of the study, the members of three leading professional organizations (International Society for Performance Improvement, The American Society for Training and Development, and Association of Educational Communication and Technology,) were invited to participate in the present study. The following research policies of these organizations were applied (See Appendix D for Letter of Supports):

- ISPI policy allowed the researcher to place a call for participants in ISPI's online newsletter www.performancexpress.org. This method would enable responses from members and non-members of ISPI. In addition, ISPI could also provide limited permission to target Certified Performance 
Technologist population by providing information and a link to the online questionnaires in ISPI's monthly CPT-exclusive email newsletter.

- ASTD policy only allowed the researcher to post a message including the invitation cover letter and a link to the questionnaires in ASTD's official discussion forums and social networks of ASTD. Moreover, ASTD encouraged using their member's directory to obtain contact information; however, the researchers were not a member of ASTD.

- AECT policy requested the researcher to submit a letter of application that must provide information about the research and the primary investigator. The executive committee and the Training and Performance Division leadership reviewed and decided to send out the survey of the current study. Once AECT provided approval, the researcher sent HIC approval and the copies of the instruments to the research director, who forwarded the invitation cover-letter to the president of Training and Performance Division. After one day, the invitation cover letter e-mailed to the all members of the division. After two weeks, the same invitation e-mail was sent out once more.

Since there was low return-rate at the beginning of the second phase data collection, the researcher attempted to reach other practitioners by using both personal contacts and professional social networking sites. For example, there were numerous groups in Facebook and Linked-In about performance improvement. A number of professional practitioners actively use these sites. As it was anticipated, these alternative options increased the return-rate of the survey. Finally, ten practitioners were 
selected randomly for the incentive, which was a \$25 Amazon.com gift card. This was another effort to increase return rate. The second phase data collection started on August 15th, 2011 and ended on October 15th, 2011. After October 15th, the survey was closed so it was no longer accessible.

In the first phase of the study, there were two separate e-mail cover letters were used (See Appendix C). The first one was invitation to the Online Ranking Questionnaire; the second one was an invitation to the Professional Profile Characteristics and the Concept-Pairs Comparison Questionnaires. Moreover, Information Sheet for Experts (See Appendix B) was used at the first round of the first phase. In the second phase, another e-mail cover letter for practitioners (See Appendix C) was used, and Information Sheet for Practitioners (See Appendix B) was used. In general, all of these cover letters and information sheets included the nature and purpose of the research project, a guarantee of anonymity, a description of the follow-up procedure for non-respondents, and the researcher's contact information, accompanied by the survey questionnaires. All of these documents were reviewed and approved by the Human Investigation Committee of Wayne State University prior to commencement of the research (See Appendix A).

Variables of the study. There are several dependent and independent variables of the current study. The dependent variables are (a) the common mental model of experts (the expert referent model) - PFNet Experts $(1, \infty)$, (b) the common novice mental mode - PFNet Novices $(1, \infty)$, and (c) the mental mode of expertise. The independent variables of the study are the following professional profile characteristics:

- Years of experience in the field, 
- $\%$ of experiences focusing on improving the participants' own performance on their profession, which is self-reported deliberate practice,

- Current industry in which the participants work,

- Number and types of different industries worked previously,

- Number of different organizations worked to date,

- Number of different HPT or HPT related projects completed to date,

- Number of different HPT or HPT related project types completed to date,

- Academic degrees and number of years spent on each degree,

- Total hours of training related to HPT,

- Number of certificates possessed to date,

- Number and types of publications made to date,

- Number of professional presentations and workshops conducted to date,

- Number of courses taught to date related to HPT,

- Number of memberships to different professional organizations,

- Number of participations to international and regional conferences,

- Years of board memberships in different professional organizations,

- Number of volunteering in different professional organizations,

- Number of awards obtained to date,

Three demographic characters, age, gender, and current job title were also used as independent variables in the current study.

\section{Data Analysis}

The Online Ranking Questionnaire results were entered into Microsoft Excel software. The frequency and percent analysis was made. Each concept was produced a 
weighted score that was used to sort the concepts in descending order. The rank number frequencies were used to calculate each concept's weighted total scores. The frequency of each concept's rank number was divided by the same rank number. For instance, if a concept had 7 selections for the first rank and 2 selections for the second rank, 7 was divided by 1 because the rank number was one; 2 was divided by 2 since the rank number was two, so on so forth. This calculation was made for the all ranking selection frequencies of the concept. The results of the divisions for all rank numbers were summed. The top 11 concepts were chosen to develop the Concept-Pairs Comparison questionnaire.

The Professional Profile Characteristics Questionnaire results for both experts and practitioners were entered into Microsoft Excel software. The data were analyzed using descriptive statistics (frequency, percent, mean, median and mode where applicable), and measures of variability (range, variance, and standard deviation where applicable). The Concept-Pairs Comparison Questionnaire results were entered into Microsoft Excel software. The 16 experts' responses to 55 concept-pair were transformed to separate text files including information about the analysis and the list of ratings. The KNOT files can only read these specific text files. In KNOT software, 16 experts' ratings scores for each 55 concept-pairs were averaged, and the common experts mental model was estimated and derived based on 11 concepts. For the common mental model of novices, 33 novices' responses to 55 concept-pair were transformed to separate text file like created for the experts. In KNOT software, 33 novices' rating scores for each 55 concept-pairs were averaged. Then the KNOT software was used to estimate and derive the common novice model. The common 
expert and novice models were also compared by using the KNOT Compare Networks Analysis. This provided similarity measures, which was also a quantitative explanation of closeness, of these two models. Chi and Koeske (1983) claimed that the density, strength and cohesiveness were the features that could be used to compare semantic representations of knowledge organizations. The density referred to the number of links; the strength referred to the repeated links between pairs of entities; the cohesiveness referred to higher order grouping and specific pattern of linkages within the representation. The similarity index covers the comparison of both the density and strength features; on the other hand, the cohesiveness is not assessed; thus, this leads the researcher to perform a qualitative comparison between the mental model graphics. Furthermore, the two mental models were compared for the similarities and differences, such as common and uncommon links.

Finally, the relatedness, coherence, and similarity scores for each expert and practitioner were calculated via the KNOT software and Pathfinder analysis and entered into SPSS software along with each expert's and practitioner's professional profile characteristics. The mental model of expertise measure was calculated by the total of these three measures. The expert referent model (the common expert model) was utilized to calculate similarity scores, and the experts' averaged ratings for each 55 concept-pair were used to calculate relatedness scores. Coherence scores were automatically calculated in the KNOT software. The relationship between professional profile characteristics and the mental model of expertise was analyzed by multiple linear regression analysis. The research questions, data sources, variables, data analysis methods for each question are illustrated in Table 3. 
Table 3. A summary of research questions

\begin{tabular}{|c|c|c|c|}
\hline Research Questions & Source of Data & Variables & Data Analysis Method \\
\hline $\begin{array}{l}\text { 1. What is the } \\
\text { common mental } \\
\text { model of expert } \\
\text { performance } \\
\text { improvement } \\
\text { practitioners? }\end{array}$ & $\begin{array}{l}\text { - The responses of } \\
16 \text { experts to the } \\
\text { Concept-Pairs } \\
\text { Comparison } \\
\text { Questionnaire }\end{array}$ & $\begin{array}{l}\text { - Dependent } \\
\text { o The common mental } \\
\text { model of experts - } \\
\text { PFNet Experts }(1, \infty)\end{array}$ & $\begin{array}{l}\text { - Average scores of } \\
16 \text { experts for each } \\
\text { comparison } \\
\text { KNOT Derive } \\
\text { Network }\end{array}$ \\
\hline $\begin{array}{l}\text { 2. What is the } \\
\text { common mental } \\
\text { model of novice } \\
\text { performance } \\
\text { improvement } \\
\text { practitioners? }\end{array}$ & $\begin{array}{l}\text { - The responses of } \\
33 \text { novices to the } \\
\text { Concept-Pairs } \\
\text { Comparison } \\
\text { Questionnaire }\end{array}$ & 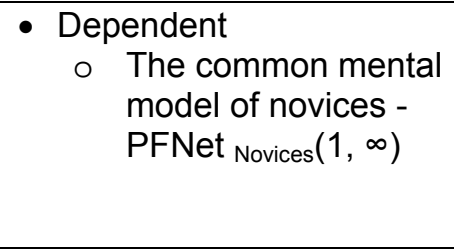 & $\begin{array}{ll}- & \text { Average scores of } \\
& 33 \text { novice } \\
\text { practitioners for } \\
\text { each comparison } \\
\text { KNOT Derive } \\
\text { Network } \\
\end{array}$ \\
\hline $\begin{array}{l}\text { 3. What, if any, are } \\
\text { the similarities and } \\
\text { differences } \\
\text { between the } \\
\text { common mental } \\
\text { models of experts } \\
\text { and novices? } \\
\end{array}$ & 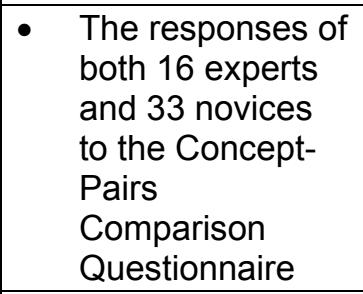 & 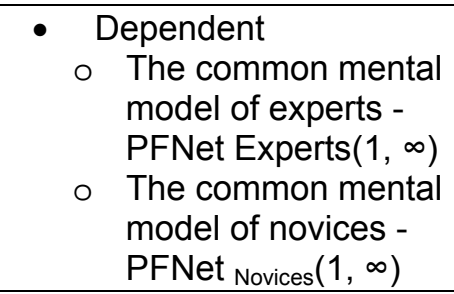 & $\begin{array}{ll}- & \text { KNOT Network } \\
& \text { Comparison - } \\
& \text { similarity scores } \\
\text { - } & \text { Graphic } \\
& \text { comparison - } \\
& \text { qualitative analysis } \\
\text { if applies } \\
\end{array}$ \\
\hline $\begin{array}{l}\text { What are the } \\
\text { professional profile } \\
\text { characteristics of } \\
\text { performance } \\
\text { improvement } \\
\text { practitioners? }\end{array}$ & $\begin{array}{l}\text { The responses of } \\
\text { both } 16 \text { experts } \\
\text { and } 273 \\
\text { practitioners to } \\
\text { the Professional } \\
\text { Profile } \\
\text { Characteristics } \\
\text { Questionnaire }\end{array}$ & $\begin{array}{ll}- & \text { Independent } \\
\text { o } & \text { All professional } \\
& \text { profile } \\
& \text { characteristics } \\
\text { o } & \begin{array}{l}\text { All demographic } \\
\text { characteristics }\end{array}\end{array}$ & $\begin{array}{l}\text { Descriptive } \\
\text { statistics: } \\
\text { frequencies, } \\
\text { percent, mean, } \\
\text { median and mode, } \\
\text { where applicable } \\
\text { Measures of } \\
\text { variability: range, } \\
\text { variance, and } \\
\text { standard deviation, } \\
\text { where applicable }\end{array}$ \\
\hline $\begin{array}{l}\text { 5. To what extent are } \\
\text { the professional } \\
\text { profile } \\
\text { characteristics of } \\
\text { performance } \\
\text { improvement } \\
\text { practitioners } \\
\text { associated with } \\
\text { their mental } \\
\text { models of } \\
\text { expertise derived } \\
\text { from Pathfinder? }\end{array}$ & $\begin{array}{l}\text { The responses of } \\
\text { both } 16 \text { experts } \\
\text { and } 273 \\
\text { practitioners to } \\
\text { the Professional } \\
\text { Profile } \\
\text { Characteristics } \\
\text { Questionnaire } \\
\text { The responses of } \\
\text { both } 16 \text { experts } \\
\text { and } 242 \\
\text { practitioners to } \\
\text { the Concept- } \\
\text { Pairs } \\
\text { Comparison } \\
\text { Questionnaire }\end{array}$ & $\begin{array}{ll}\text { - Independent } \\
\text { o All professional } \\
\text { profile } \\
\text { characteristics } \\
\text { o All demographic } \\
\text { characteristics } \\
\text { Dependent } \\
\text { o The mental model of } \\
\text { expertise derived } \\
\text { from the total of } \\
\text { relatedness, } \\
\text { coherence, and } \\
\text { similarity scores }\end{array}$ & $\begin{array}{l}\text { Multiple-linear } \\
\text { regression }\end{array}$ \\
\hline
\end{tabular}


The first and second research questions were answered by the mental model graphics of both the 16 experts and 33 novices based on their ratings in the ConceptPairs Comparison Questionnaire. Each group's rating score was averaged to obtain group mental model graphics. The coherence scores of these two models were calculated. The third question compared these two mental model graphics and obtained a similarity score, which enabled the researcher to make quantitative comparison. Each mental model graphic was also compared to each other in terms of their common and uncommon links. The fourth question was answered by the responses of both 16 experts and 273 practitioners to the Professional Profile Characteristics Questionnaire. The last question revealed the relations between each professional profile characteristic with the mental model of expertise. A multiple linear regression was performed since it allows to ". . . predict a continuous outcome from a set of variables that may be continuous, discrete, dichotomous, or a mix" (Field, 2009; Tabachnick \& Fidell, 2007). The continuous outcome in the present study was the mental model of expertise. The results of this analysis provided the best predictors of the mental model of expertise among the professional profile and demographic characteristics variables. Due to missing values and outliers, 3 experts and 73 practitioners were excluded in regression analysis. The total 212 (13 experts and 199 practitioners) participants were used.

\section{Summary}

Initially, 30 concepts were indentified after reviewing the models in the HPT field. These 30 concepts were used as items for the Online Ranking Questionnaire; it was sent out to the 58 experts selected based on the five criteria. 23 experts responded and ranked ten concepts out of 30 on the basis of their criticality for the general 
understanding of the field in the first round of the first phase. The deliverable of the first round was the 11 most important concepts. For the second round of the first phase, the same 58 experts were contacted again to complete the Professional Profile Characteristics and the Concept-Pairs Comparison Questionnaires. 16 experts responded and completed both of the questionnaires. The proximity data collected via the Concept-Pairs Comparison Questionnaire were analyzed by Knowledge Network Organizing Tool (KNOT) utilizing Pathfinder algorithm. The result of this analysis provided the deliverable of the second round of the first phase, an expert referent model - the common mental model. The first phase was applied to support the primary research activities taking into place at the second phase.

The Professional Profile Characteristics and Concept-Pairs Comparison questionnaires were sent out the members of ISPI, ASTD, and AECT Training and Performance Track by utilizing each organization's research policy. 272 practitioners completed the Professional Profile Characteristics, and 242 practitioners were completed both of the Professional Profile Characteristics and the Concept-Pairs Comparison questionnaires. 33 practitioners were identified as novices depending on the criteria used to select experts' the common novice model was produced using the KNOT software and Pathfinder analysis. The responses of 16 experts and 242 practitioners to the Concept-Pairs Comparison questionnaire were used to calculate each expert's and practitioner's relatedness, coherence, and similarity scores. The relatedness scores were calculated by the correlation between the expert referent model's concept-pair ratings and each expert's and practitioner's concept-pair ratings. The coherence scores were provided by the KNOT software for each expert and 
practitioner. The similarity scores were obtained by the comparison of each expert's and participant's mental model to the expert referent model. These three measures were used to create the mental model of expertise measure, which was a quantified explanation of each expert and practitioner's mental model. The relations between the professional profile characteristics and the mental model of expertise of both the experts and practitioners were analyzed by several multiple-linear regressions analysis. Due to missing values and outliers, 212 participants including both the experts and practitioners were used in regression analysis. 


\section{CHAPTER 4}

\section{RESULTS}

\section{Introduction}

The results of the data analyses are presented in this chapter. The chapter has seven main parts: (a) identification of concepts, (b) identification of expert referent model, (c) five research questions of the present study.

The primary purpose of this study is to reveal performance improvement practitioner expert and novice mental models and identify differences and similarities between these models. The secondary purpose is to analyze the potential impact of the demographic characteristics of performance improvement practitioners on their expertise level. The following research questions are to be addressed:

1. What is the common mental model of expert performance improvement practitioners?

2. What is the common mental model of novice performance improvement practitioners?

3. What, if any, are the similarities and differences between the common mental models of experts and novices?

4. What are the professional profile characteristics of performance improvement practitioners?

5. To what extent are the professional profile characteristics of performance improvement practitioners associated with their mental models of expertise derived from Pathfinder? 
In the present study, there were two phases. The first phase was for identification of the concepts and experts referent model; the second phase was utilized for primary research procedures. In the next section, the results of the first phase will be explained. The first round of the first phase was performed to identify the concepts necessary for both the second round of the first phase and the second phase of the study.

\section{Identification of concepts}

In the online ranking questionnaire, experts were asked to choose 10 of 30 concepts and rank them from first to tenth in terms of the concepts' criticality for the performance improvement field. An invitation e-mail which provided brief information about the research study and why they were contacted was sent out to those 57 experts. The experts were contacted twice to increase response rate if they had not already responded the survey.

Table 4 illustrates the responses to the online ranking questionnaire. Each concepts ranking frequency from one to ten is presented. The total frequency of each concept regardless their rank numbers are also presented to demonstrate how many experts chose which concepts. The rank number frequencies were used to calculate weighted total scores. The frequency of each concept in a rank number was divided by the same rank number. For instance, the concept "Focus on Results" had 7 selections for the first rank and 2 selections for the second rank. 7 was divided by 1 because the rank number was one; 2 was divided by 2 since the rank number was two, so on so forth. This calculation was made for the all ranking selection frequencies. The results of the divisions for all rank numbers were summed, and the result of this sum was listed as 
weighted total in Table 4. The higher weighted score there are, the more important concepts there are.

Table 4. Ranking, total, and weighted total scores for 30 concepts

\begin{tabular}{|c|c|c|c|c|c|c|c|c|c|c|c|c|}
\hline \multirow{2}{*}{ Concepts } & \multicolumn{10}{|c|}{ Rank } & \multirow{2}{*}{ Total } & \multirow{2}{*}{$\begin{array}{l}\text { Weighted } \\
\text { Total }\end{array}$} \\
\hline & 1 & 2 & 3 & 4 & 5 & 6 & 7 & 8 & 9 & 10 & & \\
\hline Focus on Results & 7 & 2 & 2 & 1 & 3 & 2 & 1 & 2 & 0 & 0 & $\begin{array}{c}20 \\
(87 \%)\end{array}$ & 10.24 \\
\hline Performance Analysis & 3 & 4 & 1 & 3 & 3 & 1 & 1 & 0 & 1 & 1 & $\begin{array}{c}18 \\
(78 \%)\end{array}$ & 7.20 \\
\hline Systems View & 2 & 2 & 2 & 0 & 4 & 1 & 0 & 3 & 2 & 1 & $\begin{array}{c}17 \\
(74 \%)\end{array}$ & 5.33 \\
\hline Critical Business Issue(s) & 2 & 2 & 0 & 4 & 2 & 1 & 1 & 0 & 0 & 2 & $\begin{array}{c}14 \\
(61 \%) \\
\end{array}$ & 4.91 \\
\hline Strategic Thinking & 3 & 1 & 0 & 1 & 0 & 3 & 1 & 0 & 2 & 0 & $\begin{array}{c}11 \\
(48 \%)\end{array}$ & 4.62 \\
\hline Gap Analysis & 1 & 2 & 4 & 2 & 0 & 2 & 0 & 1 & 1 & 0 & $\begin{array}{c}13 \\
(57 \%)\end{array}$ & 4.40 \\
\hline Cause Analysis & 1 & 1 & 3 & 1 & 2 & 2 & 3 & 0 & 0 & 2 & $\begin{array}{c}15 \\
(65 \%)\end{array}$ & 4.11 \\
\hline Systematic Approach & 1 & 3 & 1 & 1 & 1 & 0 & 0 & 3 & 2 & 0 & $\begin{array}{c}12 \\
(52 \%)\end{array}$ & 3.88 \\
\hline Measurement \& Evaluation & 0 & 1 & 1 & 1 & 4 & 2 & 3 & 1 & 2 & 3 & $\begin{array}{c}18 \\
(78 \%)\end{array}$ & 3.29 \\
\hline $\begin{array}{l}\text { Intervention Selection, Design \& } \\
\text { Development }\end{array}$ & 1 & 1 & 0 & 1 & 1 & 2 & 2 & 2 & 3 & 1 & $\begin{array}{c}14 \\
(61 \%)\end{array}$ & 3.25 \\
\hline Establishing Partnership with Client & 0 & 1 & 1 & 5 & 1 & 1 & 2 & 1 & 1 & 2 & $\begin{array}{c}15 \\
(65 \%) \\
\end{array}$ & 3.17 \\
\hline Needs Assessment & 1 & 0 & 2 & 2 & 0 & 0 & 2 & 1 & 1 & 0 & $\begin{array}{c}9 \\
(39 \%)\end{array}$ & 2.69 \\
\hline Work, Worker \& Workplace & 1 & 0 & 0 & 1 & 0 & 1 & 1 & 3 & 1 & 1 & $\begin{array}{c}9 \\
(39 \%)\end{array}$ & 2.15 \\
\hline Added-value & 0 & 1 & 3 & 0 & 0 & 0 & 1 & 0 & 1 & 2 & $\begin{array}{c}8 \\
(35 \%)\end{array}$ & 1.95 \\
\hline Intervention Implementation & 0 & 0 & 0 & 0 & 0 & 0 & 2 & 4 & 1 & 1 & $\begin{array}{c}8 \\
(35 \%)\end{array}$ & 1.00 \\
\hline Return on Investment & 0 & 1 & 0 & 0 & 1 & 0 & 0 & 0 & 1 & 1 & $\begin{array}{c}4 \\
(17 \%) \\
\end{array}$ & .91 \\
\hline Cost-Effectiveness & 0 & 0 & 1 & 0 & 0 & 0 & 1 & 0 & 2 & 2 & $\begin{array}{c}6 \\
(26 \%)\end{array}$ & .90 \\
\hline Instructional Design & 0 & 0 & 0 & 0 & 0 & 2 & 1 & 2 & 0 & 0 & $\begin{array}{c}5 \\
(22 \%) \\
\end{array}$ & .73 \\
\hline Ethical Dimensions & 0 & 0 & 1 & 0 & 0 & 1 & 0 & 0 & 0 & 2 & $\begin{array}{c}4 \\
(17 \%)\end{array}$ & .70 \\
\hline Training & 0 & 1 & 0 & 0 & 0 & 0 & 1 & 0 & 0 & 0 & $\begin{array}{c}2 \\
(9 \%)\end{array}$ & .64 \\
\hline Appreciative Inquiry & 0 & 0 & 1 & 0 & 0 & 0 & 0 & 0 & 0 & 0 & $\begin{array}{c}1 \\
(4 \%) \\
\end{array}$ & .33 \\
\hline Incentives \& Motives & 0 & 0 & 0 & 0 & 0 & 1 & 0 & 0 & 1 & 0 & $\begin{array}{c}2 \\
(9 \%) \\
\end{array}$ & .28 \\
\hline Knowledge \& Skills & 0 & 0 & 0 & 0 & 1 & 0 & 0 & 0 & 0 & 0 & $\begin{array}{c}1 \\
(4 \%) \\
\end{array}$ & .20 \\
\hline Financial Management & 0 & 0 & 0 & 0 & 0 & 1 & 0 & 0 & 0 & 0 & $\begin{array}{c}1 \\
(4 \%)\end{array}$ & .17 \\
\hline
\end{tabular}




\begin{tabular}{|l|c|c|c|c|c|c|c|c|c|c|c|c|}
\hline Team Development & 0 & 0 & 0 & 0 & 0 & 0 & 0 & 0 & 1 & 0 & $\begin{array}{c}1 \\
(4 \%)\end{array}$ & .11 \\
\hline Communities of Professional Practice & 0 & 0 & 0 & 0 & 0 & 0 & 0 & 0 & 0 & 1 & $\begin{array}{c}1 \\
(4 \%)\end{array}$ & .10 \\
\hline Strategic Planning & 0 & 0 & 0 & 0 & 0 & 0 & 0 & 0 & 0 & 1 & $\begin{array}{c}1 \\
(4 \%)\end{array}$ & .10 \\
\hline Individual Capacity & 0 & 0 & 0 & 0 & 0 & 0 & 0 & 0 & 0 & 0 & $\begin{array}{c}0 \\
(0 \%)\end{array}$ & .00 \\
\hline Sufficient Resources & 0 & 0 & 0 & 0 & 0 & 0 & 0 & 0 & 0 & 0 & $\begin{array}{c}0 \\
(0 \%)\end{array}$ & .00 \\
\hline Talent Management & 0 & 0 & 0 & 0 & 0 & 0 & 0 & 0 & 0 & 0 & $\begin{array}{c}0 \\
(0 \%)\end{array}$ & .00 \\
\hline
\end{tabular}

$N=23$

According to 23 experts, Focus on Results was identified as the most important concept. 20 (87\%) experts chose this concept in one of the ranks; 7 experts identified it at the first rank. The weighted total for this concept was 10.24 the highest value. Performance Analysis was the second most important concept with 7.20 weighted total score and $18(78 \%)$ selections; Systems View was the third most important concept with 5.33 weighted total and $17(74 \%)$ selections. The concepts Individual Capacity, Sufficient Resources, and Talent Management were selected by none of the experts. This means that experts were agreed that these concepts are not as critical as concepts like Focus on Results, Performance Analysis, Systems View, etc.

The online ranking scale was applied to differentiate the most critical 10 concepts for the second round of the first phase and the second phase of the study. The researcher sorted the concepts based on their weighted total scores; the concepts' total frequency numbers also were considered. After that, the first ten concepts were automatically identified. However, the eleventh concept Establishing Partnership with Client would have been eliminated if the ten concepts were selected. The researcher made a decision to include this concept to the second phase of the study. Hence, it is also an important concept for the field. Moreover, there was only .08 weighted total 
score difference from the previous concept, Intervention Selection Design and Development. Finally, all concepts on the first 10 ranks had score higher than three, and Establishing Partnership with Client had also weighted total score, 3.17. For these reasons, the researcher made aforementioned decision.

The first round of the first phase yielded the most important eleven concepts for the field of performance improvement. The selection frequencies of these eleven concepts range from $40 \%$ to more than $80 \%$; the minimum weighted total score was 3.17, and the maximum was 10.24 . The following concepts were selected after the experts' rankings:

1. Focus on Results

2. Performance Analysis

3. Systems View

4. Critical Business Issue(s)

5. Strategic Thinking

6. Gap Analysis

7. Cause Analysis

8. Systematic Approach

9. Measurement and Evaluation

10. Intervention Selection, Design and Development

11. Establishing Partnership with Client.

These concepts were used to create the Concept-Pair Comparison questionnaire (See Appendix F). As a result of selecting 11 concepts, the online ranking questionnaire had 55 pairs to compare. 
The experts also responded to an open-ended additional comments section. The main purpose for this question was to obtain other concepts that might be neglected by the researcher. If a concept was repeated numerous times by the experts in this question, it would have been considered during the selection process. Even though there were interesting responses, they were not any frequently repeated concepts.

\section{Identification of Expert Referent Model}

At the second round, the same 23 experts, responded in the first round, were contacted once more to fill the second survey including the Professional Profile Characteristics and the Concept-Pairs Comparison questionnaires. Sixteen experts $(28 \%)$ responded to the second round of the survey. In the following sections, the common mental model of experts, that is, the expert referent model, which was generated from the concept-pair questionnaire responses, are presented. The results of the common mental model will also answer the first research question of the present study.

\section{Research Question 1: What Is The Common Mental Model Of Expert Performance Improvement Practitioners?}

16 experts provided proximity data for 55 pairs of concepts selected at the first round of the study. These proximity data were analyzed by utilizing KNOT performing Pathfinder Network analysis. Sixteen experts' responses to each 55 concept-pair were averaged, and then these averaged scores were used to derive an expert referent mental model. The result of this analysis generated the model in Figure 1. 


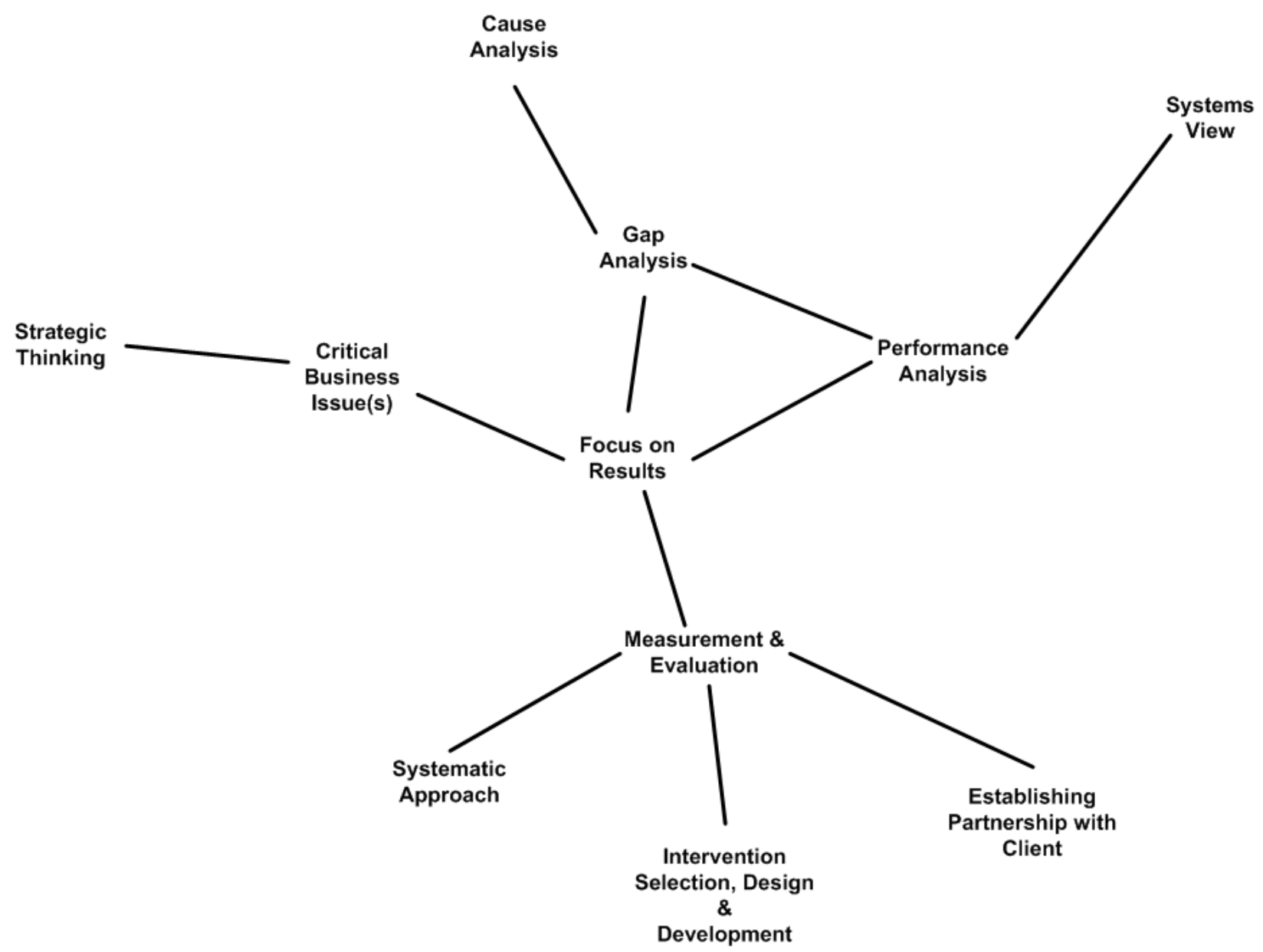

Figure 1. The expert referent model generated from 16 experts' proximity data

In this common model, coherence score, which represents internal consistency of measure, was .447. According to Villachica, et al. (2001), the value higher than .400 demonstrates the correlation with expertise or degree of learning. There are eleven nodes and eleven links. The $q$ parameter is ten, and the $r$ parameter is infinitive.

In this model, Focus on Results is at the center of experts' mental model. Gap Analysis, Performance Analysis, Measurement and Evaluation, and Critical Business Issue(s) are the secondary critical concepts branched from Focus on Results. Gap Analysis and Performance Analysis are also associated with each other. Interestingly, 
the experts' mental model connects Gap Analysis with Cause Analysis, and also, Performance Analysis is related to Systems View. Critical Business Issue(s) looks like a bridge between Focus on Results and Strategic Thinking. Measurement and Evaluation is linked with Systematic Approach, Intervention Selection, Design, and Development, and Establishing Partnership with Client. The experts' mental model states that measurement and evaluation demonstrates systematic nature of performance improvement. Moreover, it supports intervention selection, design, and development process. That might be considered formative, summative, and confirmative modes of evaluation. The most interesting result is between measurement and evaluation and establishing partnership with client. Measurement and Evaluation is the most critical process to demonstrate the impact of any interventions applied to solve performance problems or issues. For this reason, the experts' model emphasizes this linkage between these concepts.

In the second phase of the study, the practitioners in HPT field were contacted. They were asked to complete the Professional Profile Characteristics and the ConceptPairs Comparison questionnaires as the experts did in the second round of the first phase. The next section will explain the common novice model as well as providing answer for the second research question of the present study.

\section{Research Question 2: What Is The Common Mental Model Of Novice Performance Improvement Practitioners?}

33 novices were selected from 242 practitioners based on the expert selection criteria explained in the method section since those 33 novices did not have more than 10-year experiences in the field, extensive publications, and was not active in 
professional organizations. Moreover, they were not suggested by any other experts. The proximity data of these 33 novices were analyzed by KNOT utilizing Pathfinder Network Analysis. 33 novices' responses to each 55 concept-pair were averaged, and then these averaged scores were used to derive a common novice mental model. The result of this analysis generated the model in Figure 2.

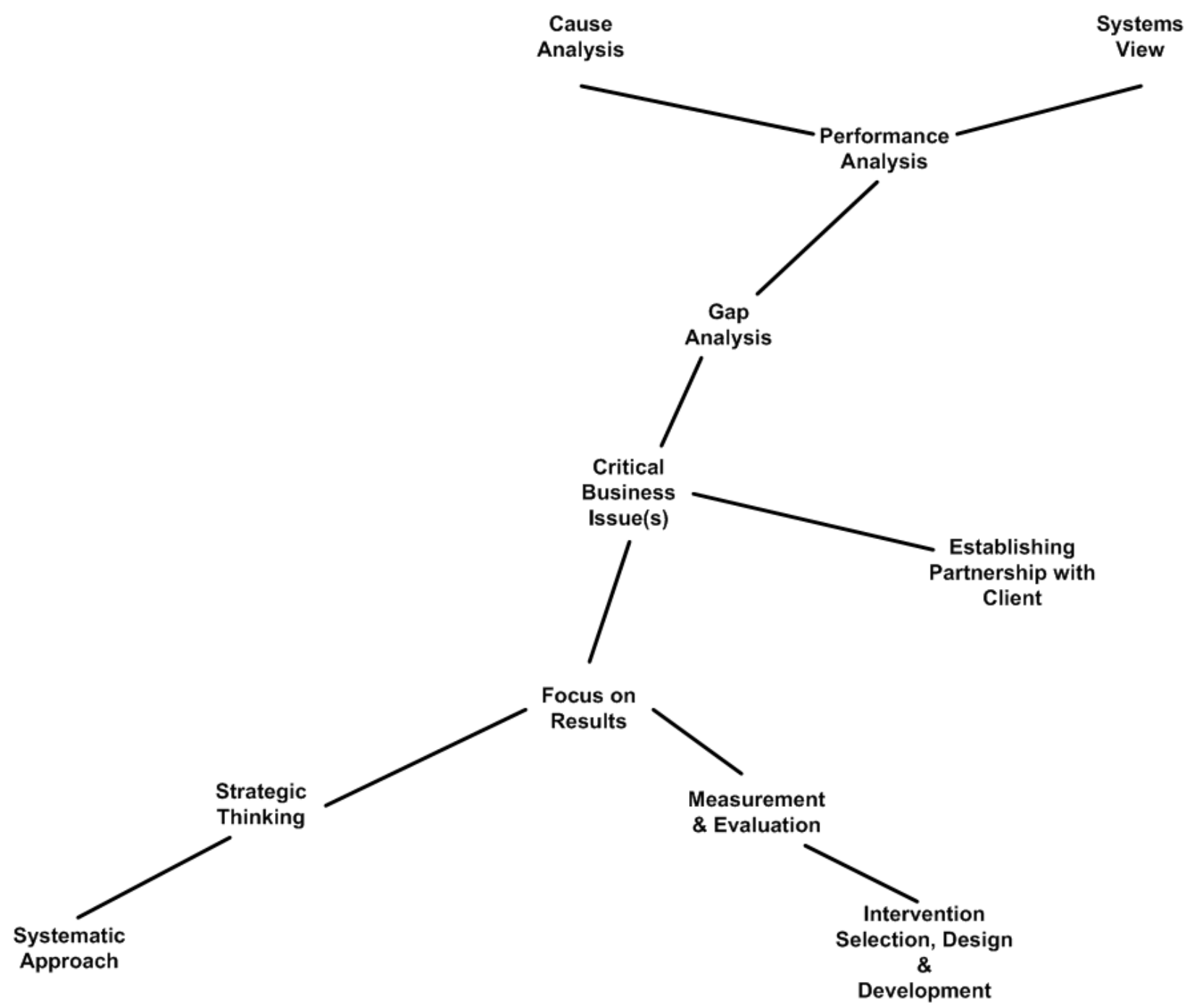

Figure 2. The common mental model of novices from 33 novices' proximity data

In this common novice model, coherence score, which represents internal consistency of measure, was .146. According to Villachica, et al. (2001), the value 
higher than .400 demonstrates the correlation with expertise or degree of learning. The coherence score also confirms that this common model is a novice model. There are ten nodes and ten links. The $q$ parameter is ten, and the $r$ parameter is infinitive.

In the common novice model, the structure looks linear. Unlike the common expert model, none of the concepts exactly are defined as the center of the model. Performance Analysis is associated with Systems View and Cause Analysis. Gap Analysis is the other side of the branching from Performance Analysis, and it connects Performance Analysis with other concepts. Gap Analysis has a connection with Critical Business Issue(s). The novice model links Critical Business Issue(s) to Establishing Partnership with Client. Focus on Results is another concept tied to Critical Business Issue(s). Focus on Results has two branching; one is Strategic Thinking, and the other one is Measurement and Evaluation. Systematic Approach is associated with Strategic Thinking; Intervention Selection, Design, \& Development is linked to Measurement and Evaluation.

Research Question 3: What, If Any, Are The Similarities And Differences Between The Common Mental Models of Experts And Novices?

The expert and novice mental models, created by the data from the first round of the first phase and the second phase respectively, were compared by using KNOT and Pathfinder Network Analysis. Figure 3 illustrates the comparison of these two models. 


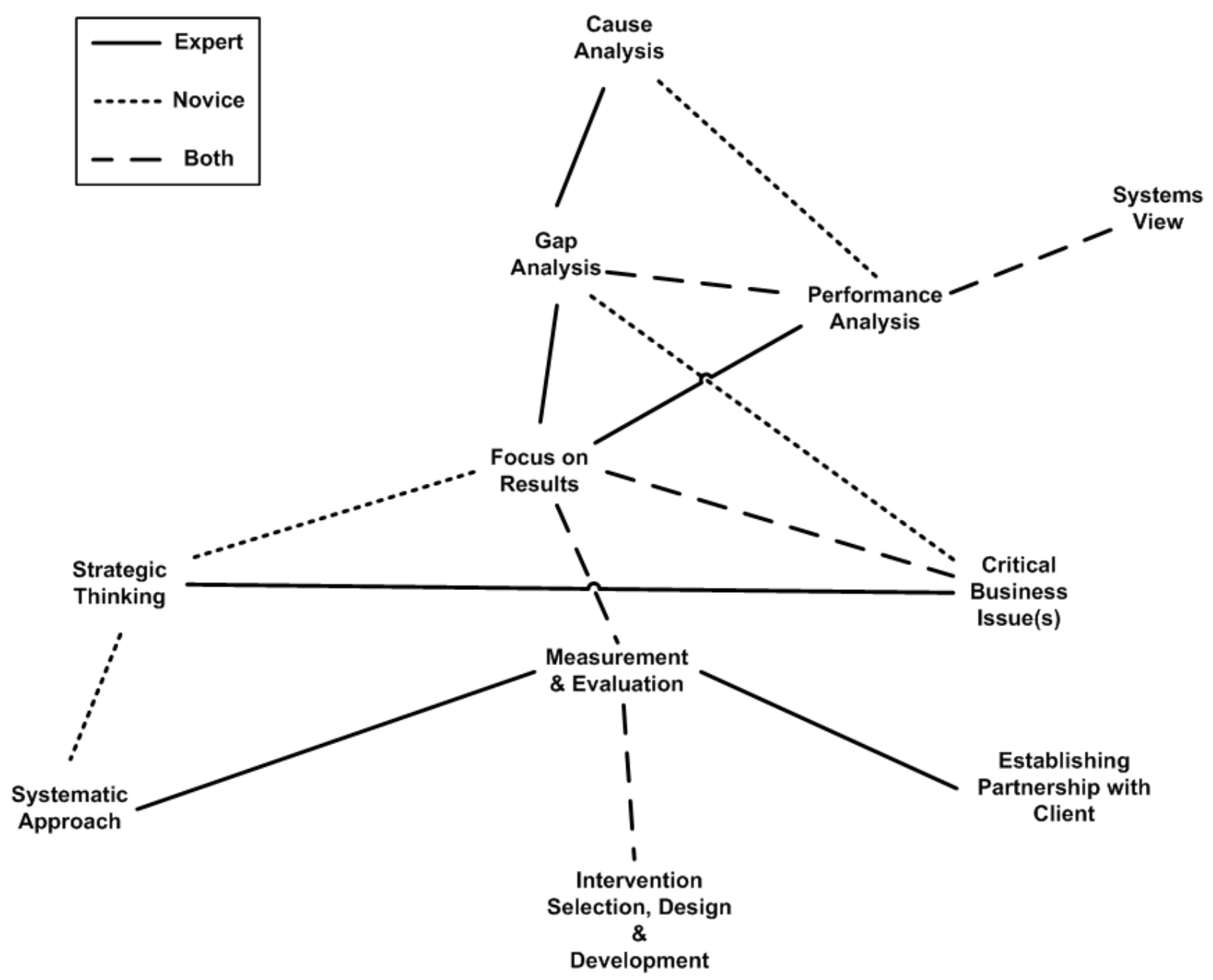

Figure 3. Comparison of the common mental models of experts and novices

There were five common links between these two models. The number of the links corrected for chance was three. The similarity measure, which is the ratio of the number of common links to the total links subtracted by the number of common links, was .313, and the similarity measure corrected for chance was .203. Similarity measures range from zero to one. Zero means no similarity or no common links; one means exact similarity or all links common. The probability of this many or more common links by chance was also provided by KNOT software. In the current 
comparison, the probability of obtaining this many or more links in common is extremely small, .0195. This measure also supports the accuracy of the similarity comparison. All of these measures state that the expert mental model of performance improvement practitioners was different from the novice practitioners. The expert mental model had higher coherence score showing that the expert mental model had more consistent and hierarchical structure. The similarity score also contended that the expert model is different from novice model about $70 \%$ to $80 \%$. The number of common links was found relatively low. Moreover, the number of common links corrected for chance was also very low. This result was another point demonstrating the difference between expert and novice models.

The following relations were the five common links in both of the mental models: (a) Performance Analysis and Systems View, (b) Performance Analysis and Gap Analysis, (c) Focus on Results and Critical Business Issue(s), (d) Focus on Results and Measurement and Evaluation, and (e) Measurement and Evaluation and Intervention Selection, Design, and Development. In the novice model, Cause Analysis is associated with Performance Analysis; whereas, the expert model links Cause Analysis to Gap Analysis. Gap Analysis connected to Critical Business Issue(s) directly in the novice model. The expert model connects Focus on Results to Critical Business Issue(s), in contrast to the novice model. Moreover, the expert model contended that Critical Business Issue(s) is also associated with Strategic Thinking, which is only linked to Focus on Results in the novice model. In the novice model, furthermore, Systematic Approach is connected to Strategic Thinking. In expert model, there is an interesting triangle linkage with three concepts: Focus on Result, Gap Analysis, and Performance 
Analysis. This might show that the experts think that these concepts are equally important and used reciprocally. In the novice model, there is nothing observed like that relationship structure. In the novice model, Measurement and Evaluation is only connected with Intervention Selection, Design, and Development. However, in the expert model, Measurement and Evaluation is also connected to Systematic Approach and Establishing Partnership with Client concepts. Experts devoted more importance to Measurement and Evaluation than novices.

The expert mental model had several core concepts and branched from these concepts. It had a more consistent and holistic structure. The novice mental model had a more linear structure, and the branching looked more supportive concepts to the concepts in the linear structure. There were no central concepts.

\section{Research Question 4: What Are The Professional Profile Characteristics Of Performance Improvement Practitioners And Experts?}

The responses of 16 experts and 273 participants, collected during the first round of the first phase and the second phase respectively, to the Professional Profile Characteristics questionnaire are presented here to answer the fourth research question. 18 experts and 335 participants started the both of the questionnaires; 16 experts $(88.9 \%)$ and $273(81.5 \%)$ practitioners completed the Professional Profile Characteristics questionnaire. The results from the practitioners are presented first, and then the results of the 16 experts are explained.

\section{The practitioners.}


Years of experience. The results from 273 practitioners are presented by questions in this section. The first question is regarding years of experience in the HPT field. Table 5 illustrates the descriptive details.

Table 5. How long have you actively been in the HPT field?

\begin{tabular}{|l|c|c|}
\hline \multicolumn{1}{|c|}{ Responses } & $\boldsymbol{n}$ & \% \\
\hline 1 to 5 years & 43 & 15.8 \\
\hline 6 to 10 years & 52 & 19.0 \\
\hline 11 to 15 years & 59 & 21.6 \\
\hline 16 to 20 years & 36 & 13.2 \\
\hline More than 20 years & 83 & 30.4 \\
\hline
\end{tabular}

$N=273$

Eighty three $(30.0 \%)$ of practitioners had more than 20 years experience in the field. About $95(34.8 \%)$ of the practitioners had experience between 11 to 20 years. Ninety five (34.8\%) had less than ten years experience in the field.

Deliberate practice. The next question is related to deliberate practice. Table 6 illustrates the descriptive details.

Table 6. The practitioners' professional activities corresponding to deliberate practice

\begin{tabular}{|l|c|c|}
\hline Responses & $\boldsymbol{n}$ & $\mathbf{\%}$ \\
\hline $0-30 \%$ & 106 & 38.82 \\
\hline $40-70 \%$ & 116 & 42.49 \\
\hline $80 \%-100 \%$ & 51 & 18.68 \\
\hline$N=273$
\end{tabular}

One hundred and six (38.82\%) practitioners stated that less than $30 \%$ of their professional activities corresponded to deliberate practice. One hundred and sixteen (42.49\%) practitioners expressed that between Forty and $70 \%$ of their professional 
activities corresponded to deliberate practice. Fifty one (18.68\%) practitioners believed that more than $80 \%$ of their professional activities were deliberate practice.

Current industry. Another question was related to the current industries in which the practitioners work. Table 7 illustrates the descriptive details from the highest to the lowest frequencies.

Table 7. What is your current industry?

\begin{tabular}{|l|c|c|}
\hline Responses & $\mathbf{n}$ & $\mathbf{\%}$ \\
\hline Independent Consultant (e.g. Performance consulting) & 43 & 15.8 \\
\hline Higher Education (College/University) & 41 & 15.0 \\
\hline Other & 34 & 12.4 \\
\hline Government & 32 & 11.7 \\
\hline Manufacturing & 18 & 6.6 \\
\hline Health Care & 15 & 5.5 \\
\hline Insurance & 12 & 4.4 \\
\hline Services & 11 & 4.0 \\
\hline Retail or Wholesale & 10 & 3.7 \\
\hline Communication and Utilities & 10 & 3.7 \\
\hline Finance & 10 & 3.7 \\
\hline Non-profit & 9 & 3.3 \\
\hline Military & 9 & 3.3 \\
\hline Internet or Information Technology & 8 & 2.9 \\
\hline K-12 Education & 5 & 1.8 \\
\hline Construction & 3 & 1.1 \\
\hline Transportation & 3 & 1.1 \\
\hline
\end{tabular}

$N=273$

Forty three (15.8\%) practitioners currently work as independent consultants. The second highest industry is Higher Education (College/University); there are 41 (15.0\%) participants. Thirty four (12.4\%) practitioners chose the "Other" industry option. Pharmaceutical, energy, food and beverage, biotechnology, aerospace, and oil and gas were the most common industries stated. The next highest industry was Government with $32(11.7 \%)$ participants. Finally, Construction and Transportation industries had the lowest numbers of practitioners, $3(1.1 \%)$. 
Number of industries worked or completed a project. The industries that the experts have worked or completed a project were the next question. The responses to this question are presented in Table 8.

Table 8. Which of the following industries have you have worked or completed a project

\begin{tabular}{|l|c|c|}
\hline Responses & $\mathbf{n}$ & $\mathbf{\%}$ \\
\hline Higher Education (College/University) & 139 & 50.9 \\
\hline Government & 121 & 44.3 \\
\hline Manufacturing & 114 & 41.8 \\
\hline Non-profit & 101 & 36.9 \\
\hline Independent Consultant (e.g. Performance consulting) & 100 & 36.6 \\
\hline Health Care & 93 & 34.1 \\
\hline Internet or Information Technology & 86 & 31.5 \\
\hline Services & 86 & 31.5 \\
\hline Finance & 75 & 27.5 \\
\hline Retail or Wholesale & 71 & 26.0 \\
\hline Military & 66 & 24.2 \\
\hline Communication and Utilities & 62 & 22.7 \\
\hline Insurance & 61 & 22.3 \\
\hline K-12 Education & 57 & 20.9 \\
\hline Transportation & 32 & 11.7 \\
\hline Construction & 30 & 10.9 \\
\hline Agriculture & 18 & 6.6 \\
\hline Real Estate & 16 & 5.9 \\
\hline Other & 31 & 11.4 \\
\hline
\end{tabular}

$\mathrm{N}=273$

One hundred and thirty nine (50.9\%) practitioners had worked or completed a project in Higher Education (College/University). The second highest industry was Government with 121 (44.3\%) participants. One hundred and fourteen (41.8\%) practitioners had experience in manufacturing industry. The next industry was non-profit organizations with $101(36.9 \%)$ practitioners. The lowest numbers of the practitioners who had worked or completed a project for Real Estate industry was $16(5.9 \%)$. 
Number of organization worked. Another question is related to the number of organizations that the practitioners have worked so far. Table 9 illustrates the responses.

Table 9. How many different organizations have you worked so far?

\begin{tabular}{|l|c|c|}
\hline Responses & $\mathbf{n}$ & $\mathbf{\%}$ \\
\hline $1-5$ & 125 & 45.78 \\
\hline $6-10$ & 87 & 31.86 \\
\hline $11-15$ & 26 & 9.50 \\
\hline $16-20$ & 11 & 4.03 \\
\hline $21-25$ & - & - \\
\hline More than 25 & 24 & 8.79 \\
\hline
\end{tabular}

Note. Dashes indicate zero

$N=273$

The majority of the practitioners $(125,45.78 \%)$ had worked one to five organizations. The second highest group, the $6-10$ organizations, had 87 practitioners $(31.86 \%)$. Twenty six practitioners had worked between 11 and 15 different organizations. There were no practitioners who have worked between 21 and 25 organizations. The last group that had 24 practitioners (8.79\%) was more than 25 organizations.

Number of projects worked. The next questions asked to estimate the total number of HPT projects that the practitioners had completed so far. The average number of completed projects was 60.32 , and the standard deviation was 136.80 . The minimum number was zero; the maximum number was 1000 . The median number of projects was 20 , and the mode number of projects was 10 . The results demonstrate the high level diversity of the completed projects so far by the participants. It is not a surprising result since performance improvement practitioners work not only on the projects directly related to HPT but also on the projects which are from other fields since 
both the theoretical and practical nature of HPT is usually acknowledged as interdisciplinary (Pershing, Lee \& Cheng, 2008a). Moreover, independent consultants might have more opportunities to work different projects simultaneously.

Types of projects worked. The kinds of HPT projects that the practitioners had worked so far were also included in the Professional Profile Characteristics Questionnaire. Table 10 illustrates the responses to this question.

Table 10. What kinds of HPT related project(s) have you worked?

\begin{tabular}{|l|c|c|}
\hline Responses & $\mathbf{n}$ & $\mathbf{\%}$ \\
\hline I have not worked on any HPT related projects & 8 & 2.9 \\
\hline Needs Assessment & 247 & 90.5 \\
\hline Performance Analysis & 220 & 80.6 \\
\hline Instructional Design & 236 & 86.4 \\
\hline Instructional Development & 227 & 83.2 \\
\hline Intervention Design \& Development & 195 & 71.4 \\
\hline Intervention Implementation & 179 & 65.6 \\
\hline Measurement and Evaluation & 225 & 82.4 \\
\hline Other & 30 & 11.0 \\
\hline
\end{tabular}

$\mathrm{N}=273$

Eight $(2.9 \%)$ practitioners stated that they had not worked on any HPT related projects. From $80 \%$ to $90 \%$ of the practitioners had experience in needs assessment, performance analysis, instructional design, instructional development, and measurement and evaluation kinds of projects. One hundred and ninety five $(70.7 \%)$ practitioners had worked in intervention design and development projects. Interestingly, only $179(65.6 \%)$ practitioners had a chance to work in intervention implementation projects. This was the lowest percent among other projects types. Thirty (11.0\%) practitioners also indicated they had worked on other types of projects. The most common projects identified under the other category were change management, competency design and development, process improvement and mapping, lean 
production, six sigma, organizational development, change, and architecture, systems design and engineering, etc.

Degrees completed or currently pursuing. A question related to the current education level and the years spent for the previous degrees were included in the questionnaire. The responses to this question are illustrated in Table 11.

Table 11. Which of the following degree(s) do you have or are you pursuing?

\begin{tabular}{|l|c|c|c|c|c|c|c|}
\hline \multicolumn{1}{|c|}{ Responses } & $\begin{array}{c}\mathbf{1} \\
\text { years }\end{array}$ & $\begin{array}{c}\mathbf{2} \\
\text { years }\end{array}$ & $\begin{array}{c}\mathbf{3} \\
\text { years }\end{array}$ & $\begin{array}{c}\mathbf{4} \\
\text { years }\end{array}$ & $\begin{array}{c}\mathbf{5} \\
\text { years }\end{array}$ & $\begin{array}{c}\text { More } \\
\text { than 5 } \\
\text { years }\end{array}$ & $\begin{array}{c}\text { Not } \\
\text { Applicable }\end{array}$ \\
\hline Associate's degree & 7 & 33 & 0 & 6 & 2 & 1 & 224 \\
\hline $\begin{array}{l}\text { Some college, no } \\
\text { degree }\end{array}$ & 3 & 4 & 4 & 1 & 0 & 2 & 259 \\
\hline Bachelor's degree & 1 & 12 & 28 & 153 & 44 & 22 & 13 \\
\hline Master's degree & 25 & 134 & 40 & 22 & 7 & 8 & 39 \\
\hline Education Specialist & 8 & 7 & 4 & 2 & 3 & 4 & 245 \\
\hline Doctorate & 4 & 20 & 22 & 12 & 9 & 25 & 181 \\
\hline Postgraduate study & 16 & 18 & 11 & 2 & 1 & 5 & 220 \\
\hline
\end{tabular}

$\mathrm{N}=273$

One hundred and fifty three (56.04\%) practitioners had spent 4 years in their bachelor's degree. One hundred and thirty four $(49.08 \%)$ practitioners stated that they had spent 2 year in their master's degrees. Ninety two (33.69\%) practitioners had spent from 1 to more than five years for their doctorate.

Training hours related to HPT. The next question is about the total estimate hours of training regarding HPT or any related activities in addition to the practitioners' degrees. The average number of training hours was 216.62 , and the standard deviation was 958.68. The minimum number was zero; the maximum number was 9999. The median number of projects was 40 , and the mode number of projects was 0 , which shows that majority of participants did not receive any training in addition to their degrees. Like the completed projects questions, the responses to this question 
demonstrated diverse results. The main reason for this might be the diversity of numerous training programs available in the current market.

Certification ownership. The certificates that the practitioners possessed also were asked in the Professional Profile Characteristics questionnaire. Table 12 illustrates the responses to this question.

Table 12. Which of the following certificates do you have?

\begin{tabular}{|l|c|c|}
\hline Responses & $\mathbf{n}$ & \% \\
\hline I don't have any certification & 146 & 53.5 \\
\hline Certified Performance Technologist (CPT) & 77 & 28.2 \\
\hline $\begin{array}{l}\text { Certified Professional in Learning and Performance } \\
\text { Certification (CPLP) }\end{array}$ & 8 & 2.9 \\
\hline Other & 79 & 28.9 \\
\hline
\end{tabular}

$N=273$

One hundred and forty six (53.5\%) practitioners stated that they did not have any certifications. Seventy seven $(28.2 \%)$ practitioners had Certified Performance Technologist designation provided by ISPI; eight (2.9\%) practitioners had Certified Professional in Learning and Performance Certification (CPLP) provided by The American Society for Training and Development. Seventy nine (28.9\%) practitioners expressed that they had other certifications. The most dominant certification observed under other category was Senior Professionals in Human Resources provided by Human Resources Certification Institute. Certification specific institutions, professional organizations (i.e. ISPI and ASTD), higher education institution programs, and widelyknown performance consulting companies (i.e. ROI institute) were the most common organizations provided the certifications that the practitioners of the present study stated. 
Number and types of publications. The publications that the practitioners authored were another question in the Professional Profile Characteristics questionnaire. Table 13 demonstrates the aggregated results of responses.

Table 13. The Number of Publications by Types

\begin{tabular}{|l|c|c|c|c|c|c|c|}
\hline Responses & $\mathbf{0}$ & $\mathbf{1 - 5}$ & $\mathbf{6 - 1 0}$ & $\mathbf{1 1 - 1 5}$ & $\mathbf{1 6 - 2 0}$ & $\mathbf{2 1 - 2 5}$ & $\begin{array}{c}\text { More } \\
\text { than } \\
\mathbf{2 5}\end{array}$ \\
\hline Books & 236 & $\mathbf{2 8}$ & 5 & 2 & 1 & - & 1 \\
\hline Book Chapters & 208 & 53 & 6 & 3 & - & - & 3 \\
\hline Refereed journals & 199 & 55 & 4 & 4 & 4 & - & 7 \\
\hline Non-refereed journals & 184 & 61 & 11 & 5 & 6 & - & 6 \\
\hline Proceedings & 199 & 44 & 15 & 4 & 3 & - & 8 \\
\hline Poster sessions & 198 & 56 & 12 & 2 & 2 & - & 3 \\
\hline $\begin{array}{l}\text { Educational/Instructional } \\
\text { Materials }\end{array}$ & 140 & 44 & 24 & 4 & 1 & 1 & 59 \\
\hline Book reviews & 210 & 48 & 7 & 5 & 1 & - & 2 \\
\hline
\end{tabular}

Note. Dashes indicate zero

$\mathrm{N}=273$

The majority of the practitioners ranging from $51.28 \%$ to $86.08 \%$ did not have any publications in one of the publication types listed. Book chapters (19.04\%), refereed journals $(20.51 \%)$, non-refereed journals $(21.97 \%)$, and posters sessions $(20.87 \%)$ were the most popular publications made ranging from one to five times. Educational/Instructional materials were published by $24(8.79 \%)$ practitioners from six to ten times. Moreover, fifty nine $(21.61 \%)$ practitioners stated that they published Educational/Instructional materials more than 25 times.

Number of professional presentations and workshops. The professional presentations and workshops that the practitioners performed were also included in the Professional Profile Characteristics questionnaire. The results are presented in Table 14. 
Table 14. How many professional presentations (i.e., how-to, educational/lecture, case study, business, futurecasting, issues, etc.) or workshops have you done so far?

\begin{tabular}{|l|c|c|c|c|c|c|c|}
\hline Responses & $\mathbf{0}$ & $\mathbf{1 - 5}$ & $\mathbf{6}-\mathbf{1 0}$ & $\mathbf{1 1}-\mathbf{1 5}$ & $\mathbf{1 6 - 2 0}$ & $\mathbf{2 1 - 2 5}$ & $\begin{array}{c}\text { More } \\
\text { than 25 }\end{array}$ \\
\hline Presentations & 31 & 84 & 52 & 17 & 7 & 1 & 81 \\
\hline Workshops & 73 & 83 & 26 & 12 & 6 & 3 & 70 \\
\hline
\end{tabular}

$\mathrm{N}=273$

Eighty one (29.67\%) and seventy (25.64\%) practitioners performed more than 25 presentations and workshops respectively. Thirty one (11.35\%) and seventy three (26.73\%) practitioners had done neither presentations nor workshops correspondingly. Eighty four $(30.76 \%)$ practitioners conducted presentations from one to five times; eighty three (30.40\%) practitioners conducted workshops from one to five times.

Number and formats of courses taught. The practitioners also stated the courses related to HPT which they taught. The question also asked for the modes of the courses, such as in class, on-line, and blended. The results of this question are illustrated in Table 15.

Table 15. The number of courses that the practitioners taught by course formats

\begin{tabular}{|l|c|c|c|c|c|c|c|}
\hline Responses & $\mathbf{0}$ & $\mathbf{1 - 5}$ & $\mathbf{6}-\mathbf{1 0}$ & $\mathbf{1 1}-\mathbf{1 5}$ & $\mathbf{1 6}-\mathbf{2 0}$ & $\mathbf{2 1 - 2 5}$ & $\begin{array}{c}\text { More } \\
\text { than 25 }\end{array}$ \\
\hline In class & 153 & 74 & 19 & 7 & 2 & 1 & 17 \\
\hline On-line & 205 & 49 & 11 & 2 & 2 & - & 4 \\
\hline Blended & 211 & 43 & 11 & 2 & 1 & - & 5 \\
\hline
\end{tabular}

Note. Dashes indicate zero

$N=273$

One hundred and fifty three $(56.04 \%)$, two hundred and five $(75.09 \%)$, and two hundred and eleven (77.29\%) practitioners had never taught courses in class, online, and blended formats in that order. Seventy three $(27.10 \%)$ practitioners had taught courses in class format between one to five times; forty nine $(17.94 \%)$ practitioners had taught online courses from one to five times; forty four (15.75\%) practitioners had taught 
blended courses from one to five times. Seventeen (6.23\%) practitioners had taught in class (face to face) courses more than 25 times.

Membership to professional organizations. The practitioners provided their membership to the professional organizations. The results of this are presented in Table 16.

Table 16. Membership to professional organizations

\begin{tabular}{|l|c|c|}
\hline Responses & $\mathbf{n}$ & $\mathbf{\%}$ \\
\hline ISPI - International & 222 & 81.3 \\
\hline ISPI - Local chapter & 83 & 30.4 \\
\hline ASTD - International & 118 & 43.2 \\
\hline ASTD - Local chapter & 70 & 25.6 \\
\hline AECT - International & 21 & 7.7 \\
\hline AECT - Local chapter & 4 & 1.5 \\
\hline No membership & 10 & 3.7 \\
\hline Other & 70 & 25.6 \\
\hline
\end{tabular}

$N=273$

Two hundred and twenty two (81.3\%) practitioners were a member of ISPI International; one hundred and eighteen (43.2\%) practitioners were members of ASTD - International. The numbers are lower in the local chapter of the same organizations. Eighty three (30.4\%) practitioners were a member of one of the ISPI local chapters; seventy (25.6\%) practitioners were a member of one of the ASTD local chapters. Seventy $(25.6 \%)$ practitioners indicated membership to other organizations. The most common one under this category was Society for Human Resource Management. Ten $(3.7 \%)$ practitioners expressed that they did not have any membership.

Participation to conferences of professional organizations. The practitioners were also asked for their participation to the conferences of the professional organizations. The results are illustrated in Table 17. 
Table 17. The number of the participations to the conferences

\begin{tabular}{|c|c|c|c|c|c|c|c|}
\hline Responses & $1-5$ & $6-10$ & $11-15$ & $16-20$ & $21-25$ & $\begin{array}{c}\text { More } \\
\text { than 25 }\end{array}$ & $\begin{array}{c}\text { Not } \\
\text { Participated }\end{array}$ \\
\hline $\begin{array}{l}\text { ISPI-The } \\
\text { Annual } \\
\text { Performance } \\
\text { Improvement } \\
\text { Conference }\end{array}$ & 124 & 23 & 8 & 6 & 2 & 4 & 106 \\
\hline $\begin{array}{l}\text { ISPI - The } \\
\text { local chapter } \\
\text { conferences, } \\
\text { seminars, \& } \\
\text { workshops }\end{array}$ & 70 & 24 & 10 & 9 & 3 & 21 & 135 \\
\hline $\begin{array}{l}\text { ASTD - Annual } \\
\text { International } \\
\text { Conference \& } \\
\text { Exposition }\end{array}$ & 101 & 18 & 6 & 0 & 3 & 5 & 140 \\
\hline $\begin{array}{l}\text { ASTD - The } \\
\text { local chapter } \\
\text { conferences, } \\
\text { seminars, \& } \\
\text { workshops }\end{array}$ & 79 & 31 & 11 & 6 & 2 & 14 & 130 \\
\hline $\begin{array}{l}\text { AECT - Annual } \\
\text { International } \\
\text { Convention }\end{array}$ & 18 & 4 & 2 & 1 & 1 & 10 & 237 \\
\hline $\begin{array}{l}\text { AECT - The } \\
\text { local chapter } \\
\text { conferences, } \\
\text { seminars, \& } \\
\text { workshops }\end{array}$ & 7 & 2 & 0 & 2 & 1 & 12 & 249 \\
\hline
\end{tabular}

Note. Dashes indicate zero

$N=273$

The majority of practitioners ranging from $38.88 \%$ to $91.20 \%$ had never participated in the conferences listed above. One hundred and twenty four (45.42\%) practitioners had participated in ISPI - The Annual Performance Improvement Conferences one to five times; one hundred and one (36.99\%) practitioners had participated in ASTD - Annual International Conference and Expositions one to five 
times. Twenty one $(7.69 \%)$ practitioners stated that they participated in more than 25 ISPI local chapter conferences, seminars, and workshops.

Board membership in professional organizations. The practitioners' board membership was also asked in the Professional Profile Characteristics questionnaire. The results of this question are presented in Table 18.

Table 18. Board membership in professional organizations

\begin{tabular}{|l|c|c|c|c|c|c|c|}
\hline \multicolumn{1}{|c|}{ Responses } & $\mathbf{1}$ & $\mathbf{2}$ & $\mathbf{3}$ & $\mathbf{4}$ & $\mathbf{5}$ & $\begin{array}{c}\text { More } \\
\text { than } \\
\mathbf{5}\end{array}$ & $\begin{array}{c}\text { Not a } \\
\text { board } \\
\text { member }\end{array}$ \\
\hline $\begin{array}{l}\text { ISPI - } \\
\text { International }\end{array}$ & 3 & 3 & 1 & 3 & 2 & 1 & 260 \\
\hline $\begin{array}{l}\text { ISPI - Local } \\
\text { chapter }\end{array}$ & 9 & 12 & 7 & 9 & 5 & 16 & 215 \\
\hline $\begin{array}{l}\text { ASTD - } \\
\text { International }\end{array}$ & 1 & 1 & 2 & 4 & 2 & - & 263 \\
\hline $\begin{array}{l}\text { ASTD - Local } \\
\text { chapter }\end{array}$ & 6 & 9 & 9 & 7 & - & 10 & 232 \\
\hline $\begin{array}{l}\text { AECT - } \\
\text { International }\end{array}$ & 2 & - & - & 3 & 4 & 2 & 262 \\
\hline $\begin{array}{l}\text { AECT - Local } \\
\text { chapter }\end{array}$ & 1 & 1 & - & 2 & 2 & 2 & 265 \\
\hline
\end{tabular}

Note. Dashes indicate zero

$N=273$

The majority of the practitioners did not have a board membership in the listed organizations above. This is an expected result. Thirteen (4.76\%) practitioners were a board member between one to five years in ISPI - International; fifty eight (21.24\%) practitioners were a board member one to five years in one of the ISPI local chapters. ten $(3.66 \%)$ practitioners served as a board member one to five years in ASTD International; forty one (15.01\%) practitioners served as a board member one to five years in one of ASTD local chapters. 
Volunteering in professional organizations. The practitioners' volunteer activities were also included in the Professional Profile Characteristics questionnaire. The results of this question are presented in Table 19.

Table 19. Volunteer activities in the professional organizations

\begin{tabular}{|l|c|c|c|c|c|c|c|}
\hline \multicolumn{1}{|c|}{ Responses } & $\mathbf{1}$ & $\mathbf{2}$ & $\mathbf{3}$ & $\mathbf{4}$ & $\mathbf{5}$ & $\begin{array}{c}\text { More } \\
\text { than } \\
\mathbf{5}\end{array}$ & $\begin{array}{c}\text { Not } \\
\text { Volunteered }\end{array}$ \\
\hline $\begin{array}{l}\text { ISPI - } \\
\text { International }\end{array}$ & 25 & 14 & 10 & 5 & 3 & 37 & 179 \\
\hline $\begin{array}{l}\text { ISPI - Local } \\
\text { chapter }\end{array}$ & 18 & 13 & 8 & 3 & 7 & 37 & 187 \\
\hline $\begin{array}{l}\text { ASTD - } \\
\text { International }\end{array}$ & 12 & 7 & 6 & 2 & 3 & 11 & 232 \\
\hline $\begin{array}{l}\text { ASTD - Local } \\
\text { chapter }\end{array}$ & 14 & 13 & 5 & 4 & 6 & 27 & 204 \\
\hline $\begin{array}{l}\text { AECT - } \\
\text { International }\end{array}$ & 2 & 2 & 1 & 3 & 1 & 6 & 258 \\
\hline $\begin{array}{l}\text { AECT - Local } \\
\text { chapter }\end{array}$ & 2 & 0 & 0 & 1 & 2 & 2 & 266 \\
\hline
\end{tabular}

Note. Dashes indicate zero

$N=273$

The majority of practitioners did not volunteered in the listed organizations. Fifty seven $(20.88 \%)$ practitioners volunteered between one to five times in ISPI International; forty nine (17.94\%) practitioners volunteered one to five times in one of the ISPI local chapters. Thirty seven (13.55\%) practitioners volunteered between more than five times in ISPI - International; Thirty seven (13.55\%) practitioners volunteered more than five times in one of the ISPI local chapters.

Number and types of awards. The practitioners were asked to provide the numbers of awards they obtained due to their professional activities in the HPT field. Two hundred and four (74.72\%) practitioners expressed that they had never received awards. Thirty three (12.08\%) practitioners stated that they earned awards from ISPI. The awards were: (a) Awards of Excellence, (b) ISPI Presidential Citation, (c) ISPI 
Chapter Excellence, (d) ISPI Service Awards, (e) ISPI Volunteer Awards, (f) ISPI Local Chapter Awards, (g) ISPI Case Study Competition Winner, (h) ISPI Distinguished Doctoral Dissertation, and (I) ISPI Research Awards. One of those 30 ISPI awards recipients stated that (s)he got his/her awards when ISPI was National Society for Performance Improvement (NSPI). Six (2.19\%) practitioners stated that they obtained ASTD related awards. The rewards were: (a) ASTD BEST Award, (b) ASTD Local Chapter Awards, (c) ASTD Excellence Awards, and (d) ASTD Outstanding Service Awards. Thirty three (12.08\%) practitioners expressed the awards that they obtained from different professional organizations or the corporations that they are currently working or have worked before. The awards were usually about recognition or outstanding performance.

Job titles. The job titles of the practitioners were also asked in the Professional Profile Characteristics questionnaire. The results are presented in Table 20. The initial responses to this question were highly diversified. For this reason, the researcher created categories for the common job titles. Some job titles had very low number of responses. They were grouped as others.

Table 20. The grouped job titles of the participants

\begin{tabular}{|l|c|c|}
\hline \multicolumn{1}{|c|}{ Job Titles } & $\mathbf{n}$ & $\mathbf{\%}$ \\
\hline Consultant & 50 & 18.32 \\
\hline Instructional Design \& Development & 31 & 11.36 \\
\hline Training \& Development & 27 & 9.89 \\
\hline Manager/Supervisor/Director & 26 & 9.52 \\
\hline Academic & 22 & 8.06 \\
\hline Executive & 22 & 8.06 \\
\hline Education & 21 & 7.69 \\
\hline Learning \& Development & 19 & 6.96 \\
\hline Performance Improvement & 13 & 4.76 \\
\hline Human Resources & 9 & 3.30 \\
\hline Others & 33 & 12.09 \\
\hline
\end{tabular}

$N=273$ 
Fifty (18.32\%) practitioners stated their job titles as Consultant. In this category, the most common job titles were independent, performance, senior, training, learning, and education consultants. Thirty one $(11.36 \%)$ practitioners expressed that their job titles were related to instructional design and development. The common job titles under this category were instructional designer, instructional developer, instructional systems designer, instructional technologist, and course design and developer. Twenty seven $(9.89 \%)$ practitioners indicated that their job titles were related to training and development. The most common job titles were training manager, training and development specialist, training and design, and training director. Twenty six (9.52\%) practitioners responded that their job titles were related to Manager, Supervisor or Director. The most common job titles under this category were general manager, director, project manager, principal, supervisor, chair, and head. Two job categories Academic and Executive had twenty two (8.06\%) practitioners for each. The common job titles for academic were assistant professor, associate professor, professor, and adjunct faculty and for executive were president, vice president, owner, partner, chief executive officer, and chief learning officer.

Age. Age was another demographic characteristic collected with the Professional Profile Characteristics questionnaire. The distributions of the practitioners' ages are illustrated in Table 21. 
Table 21. The practitioners' ages

\begin{tabular}{|l|c|c|}
\hline Responses & $\mathbf{n}$ & $\mathbf{\%}$ \\
\hline Less than 30 & 13 & 4.8 \\
\hline $31-40$ & 35 & 12.8 \\
\hline $41-50$ & 80 & 29.3 \\
\hline $51-60$ & 104 & 38.1 \\
\hline $61-70$ & 37 & 13.6 \\
\hline $71-80$ & 3 & 1.1 \\
\hline More than 80 & 1 & 0.4 \\
\hline
\end{tabular}

The majority of practitioners $(104,38.1 \%)$ were between the ages of 51 and 60 . The second most crowded group was between the ages of 41 to 50 with 80 (29.3\%) practitioners. There was only one person who was more than 80 years old.

Gender. The second demographic characteristic collected with the Professional Profile Characteristics questionnaire was gender. The results of the practitioners' gender distribution are presented in Table 22.

Table 22. The practitioners' gender

\begin{tabular}{|l|c|c|}
\hline Responses & $\mathbf{n}$ & $\mathbf{\%}$ \\
\hline Male & 126 & 46.2 \\
\hline Female & 147 & 53.8 \\
\hline $\mathrm{N}=273$
\end{tabular}

One hundred and twenty six (46.2\%) practitioners were male, and one hundred and forty seven (53.8\%) practitioners were female. The number of female practitioners was higher than male practitioners.

The experts. The Professional Profile Characteristics were also shared by the sixteen experts who participated in the second round of the first phase of the study.

Years of experience. The first question is regarding years of experience of experts in the HPT field. Table 23 illustrates the descriptive details. 
Table 23. How long have you actively been in the HPT field?

\begin{tabular}{|l|c|c|}
\hline \multicolumn{1}{|c|}{ Responses } & $\mathbf{n}$ & $\mathbf{\%}$ \\
\hline 11 to 15 years & 3 & 18.75 \\
\hline More than 20 years & 13 & 81.25 \\
\hline
\end{tabular}

$\mathrm{N}=16$

Three (18.75\%) experts had experience in the field from 11 to 15 years. 13 (81.25\%) experts had experience more than 20 years. All experts had experience more than ten years, which was indicated as only empirical evidence for expertise (Ericsson \& Charness, 1994).

Deliberate practice. The next question is related to deliberate practice. Table 24 illustrates the descriptive details.

Table 24. The experts' professional activities corresponding to deliberate practice

\begin{tabular}{|l|c|c|}
\hline Responses & $\mathbf{n}$ & $\mathbf{\%}$ \\
\hline $0-30 \%$ & 5 & 31.25 \\
\hline $40-70 \%$ & 9 & 56.25 \\
\hline $80 \%-100 \%$ & 2 & 12.50 \\
\hline $\mathrm{N}=16$
\end{tabular}

Nine (56.25\%) experts stated that between 40 and $70 \%$ of their experiences may be identified as deliberate practice. Five (31.25\%) experts expressed that only less than $30 \%$ of their professional practices may be accepted as deliberate practice. Two $(12.50 \%)$ experts believed that more than $80 \%$ of their professional activities were deliberate practice.

Current industry. Experts shared the industries in which they currently work. Table 25 demonstrates the results.. 
Table 25. The experts' current industries

\begin{tabular}{|l|c|c|}
\hline Responses & $\mathbf{n}$ & $\mathbf{\%}$ \\
\hline Independent Consultant (e.g. Performance consulting) & 9 & 56.25 \\
\hline Higher Education (College/University) & 5 & 31.25 \\
\hline Manufacturing & 1 & 6.25 \\
\hline Defense Industry & 1 & 6.25 \\
\hline
\end{tabular}

$\mathrm{N}=16$

Nine $(56.25 \%)$ experts stated that they currently work as an independent consultant.

Five (31.25\%) identified their current industry as higher education, especially colleges or universities. One (6.25\%) expert stated that (s)he work in a manufacturing industry. Another (6.25\%) expert expressed that they work at defense industry..

Industries have worked or completed a project. The industries that the experts have worked or completed a project were another question. The responses to this question are presented in Table 26.

Table 26. The experts' industries in which they have worked or completed a project

\begin{tabular}{|l|c|c|}
\hline Responses & $\mathbf{n}$ & $\%$ \\
\hline Higher Education (College/University) & 13 & 81.25 \\
\hline Government & 12 & 75.00 \\
\hline Independent Consultant (e.g. Performance consulting) & 12 & 75.00 \\
\hline Health Care & 12 & 75.00 \\
\hline Military & 11 & 68.75 \\
\hline Non-profit & 10 & 62.50 \\
\hline Finance & 10 & 62.50 \\
\hline Retail or Wholesale & 10 & 62.50 \\
\hline K-12 Education & 10 & 62.50 \\
\hline Manufacturing & 9 & 56.25 \\
\hline Insurance & 9 & 56.25 \\
\hline Internet or Information Technology & 8 & 50.00 \\
\hline Services & 8 & 50.00 \\
\hline Communication and Utilities & 8 & 50.00 \\
\hline Transportation & 4 & 25.00 \\
\hline Real Estate & 3 & 18.75 \\
\hline Other & 2 & 12.50 \\
\hline Construction & - & - \\
\hline Agriculture & - & - \\
\hline
\end{tabular}

Note. Dashes indicate zero

$\mathrm{N}=16$ 
The majority of experts $(13,81.25 \%)$ had worked in higher education. Twelve $(75.00 \%)$ experts indicated that they had worked in government, independent consultancy, and health care industries. $11(68.75 \%)$ experts expressed that they had military experiences. Ten (62.50\%) experts stated that they worked in non-profit, finance, retail or wholesale, and K-12 education institutions. The experts had no experience in construction and agriculture industries.

Number of organization worked. Another question is related to the number of organizations that the participants have worked so far. Table 27 illustrates the responses.

Table 27. The number of organizations which experts worked

\begin{tabular}{|l|c|c|}
\hline Responses & $\mathbf{n}$ & $\mathbf{\%}$ \\
\hline $1-5$ & 3 & 18.75 \\
\hline $6-10$ & 2 & 12.50 \\
\hline More than 25 & 11 & 68.75 \\
\hline
\end{tabular}

Note. Dashes indicate zero $N=16$

The majority of the experts $(11,68.75 \%)$ had worked in more than 25 organizations. Three $(18.75 \%)$ experts had worked in one to five different organizations. Finally, two $(12.50 \%)$ experts had worked in six to ten different organizations. These results demonstrate the experts' many experiences in working with different organizations.

Number of projects worked. The next questions asked to estimate the total number of HPT projects that the experts had completed so far. The average number of completed projects was 134.19 , and the standard deviation was 137.11 . The minimum number was seven; the maximum number was 500 . The median number of projects was 100 , and the mode number of projects was 100 . The results demonstrate the high level diversity of the completed projects so far by the experts. The average number of 
experts' projects was higher than the participants' average number $(M=60.97, S D=$ 137.04).

Types of projects worked. The kinds of HPT projects that the experts had worked so far were also included in the Professional Profile Characteristics Questionnaire. Table 28 illustrates the responses to this question.

Table 28. The types of projects that the experts worked

\begin{tabular}{|l|c|c|}
\hline Responses & $\mathbf{n}$ & $\mathbf{\%}$ \\
\hline I have not worked on any HPT related projects & - & - \\
\hline Needs Assessment & 16 & 100.00 \\
\hline Performance Analysis & 15 & 93.75 \\
\hline Instructional Design & 15 & 93.75 \\
\hline Instructional Development & 15 & 93.75 \\
\hline Intervention Design \& Development & 14 & 87.50 \\
\hline Intervention Implementation & 14 & 87.50 \\
\hline Measurement and Evaluation & 15 & 93.75 \\
\hline Other & 3 & 18.75 \\
\hline
\end{tabular}

Note. Dashes indicate zero

$\mathrm{N}=16$

All of the experts had experience in needs assessment. Fifteen (93.75\%) experts had worked in performance analysis, instructional design, instructional development, and measurement and evaluation kinds of projects. Fourteen $(87.50 \%)$ experts had worked in intervention design and development and intervention implementation projects. The expert group had a higher percentage than the participants ( $n=179$, $65.6 \%)$ in intervention implementation projects. Three $(11.0 \%)$ participants also indicated they had worked on other types of projects. The most common projects identified under the other category were training systems engineering, process redesign, pay for performance compensation, recruiting and selection systems, organization and job redesign, staffing and career planning systems, performance 
appraisal and management systems, change management, personnel development, document design, and policy redesign.

Degrees completed or currently pursuing. The current education level and the years spent for the degrees were tabulated. The responses to this question are illustrated in Table 29.

Table 29. The degree completed or currently being pursued by the experts

\begin{tabular}{|l|c|c|c|c|c|c|c|}
\hline \multicolumn{1}{|c|}{ Responses } & $\begin{array}{c}\mathbf{1} \\
\text { years }\end{array}$ & $\begin{array}{c}\mathbf{2} \\
\text { years }\end{array}$ & $\begin{array}{c}\mathbf{3} \\
\text { years }\end{array}$ & $\begin{array}{c}\mathbf{4} \\
\text { years }\end{array}$ & $\begin{array}{c}\mathbf{5} \\
\text { years }\end{array}$ & $\begin{array}{c}\text { More } \\
\text { than 5 } \\
\text { years }\end{array}$ & $\begin{array}{c}\text { Not } \\
\text { Applicable }\end{array}$ \\
\hline Associate's degree & - & 2 & - & - & - & - & 14 \\
\hline $\begin{array}{l}\text { Some college, no } \\
\text { degree }\end{array}$ & - & - & - & - & - & - & 16 \\
\hline Bachelor's degree & & 1 & 2 & 11 & 2 & - & - \\
\hline Master's degree & 4 & 7 & 2 & - & - & 1 & 2 \\
\hline Education Specialist & - & 2 & - & - & - & - & 14 \\
\hline Doctorate & - & 2 & 7 & 4 & 1 & 1 & 1 \\
\hline Postgraduate study & 1 & 2 & - & - & - & 1 & 12 \\
\hline
\end{tabular}

Note. Dashes indicate zero

$\mathrm{N}=16$

Eleven $(68.75 \%)$ participants had spent 4 years in their bachelor's degree. Seven $(43.75 \%)$ participants stated that they had spent 2 year in their master's degrees. Fifteen $(93.75 \%)$ participants had spent from two to more than five years for their doctorate.

Training hours related to HPT. The next question is about the total estimate hours of training regarding HPT or any related activities in addition to the experts' degrees. The average number of training hours was 166.13 , and the standard deviation was 296.05. The minimum number was zero; the maximum number was 1000 . The median number of projects was 45 , and the mode number of projects was 0 . Similar to 
the completed projects questions, the responses to this question demonstrated diverse results. The main reason for this might be the diversity of numerous training programs available in the current market. The experts' estimated training hours was lower than the participants' training hours $(M=216.95, S D=958.63)$. However, the participants' standard deviation for the training hours was higher than the experts. This result was obtained since the majority of the experts had completed or have currently pursued doctorate work.

Certification ownership. The certificates that the experts had were also asked in the Professional Profile Characteristics questionnaire. Table 30 illustrates the responses to this question.

Table 30. The certification ownership of the experts

\begin{tabular}{|l|c|c|}
\hline Responses & $\mathbf{n}$ & \% \\
\hline I don't have any certification & 3 & 18.75 \\
\hline Certified Performance Technologist (CPT) & 12 & 75.00 \\
\hline $\begin{array}{l}\text { Certified Professional in Learning and Performance } \\
\text { Certification (CPLP) }\end{array}$ & 2 & 12.50 \\
\hline Other & 5 & 31.25 \\
\hline
\end{tabular}

$N=16$

Three $(18.75 \%)$ experts stated that they did not have any certifications. Twelve (75.00\%) experts had Certified Performance Technologist designation provided by ISPI; $2(12.50 \%)$ experts had Certified Professional in Learning and Performance Certification (CPLP) provided by The American Society for Training and Development. Five $(31.25 \%)$ participants expressed that they had other certifications. The other certifications were related to $\mathrm{K}-12$, management, environmental organizations, etc. The most HPT relevant one was Certified ROI Professional. 
Number and types of publications. The publications that the experts had made were another question in the Professional Profile Characteristics questionnaire. Table 31 demonstrates the aggregated results of responses.

Table 31. The Number of Publications by Types

\begin{tabular}{|l|c|c|c|c|c|c|c|}
\hline Responses & $\mathbf{0}$ & $\mathbf{1 - 5}$ & $\mathbf{6 - 1 0}$ & $\mathbf{1 1 - 1 5}$ & $\mathbf{1 6}-\mathbf{2 0}$ & $\mathbf{2 1 - 2 5}$ & $\begin{array}{c}\text { More } \\
\text { than } \mathbf{2 5}\end{array}$ \\
\hline Books & 3 & 6 & 3 & 2 & - & - & 2 \\
\hline Book Chapters & 2 & 6 & 4 & 2 & - & - & 2 \\
\hline Refereed journals & 6 & 4 & 1 & 2 & - & - & 3 \\
\hline $\begin{array}{l}\text { Non-refereed } \\
\text { journals }\end{array}$ & - & 3 & 4 & - & 1 & - & 8 \\
\hline Proceedings & 6 & 4 & - & 1 & - & - & 5 \\
\hline Poster sessions & 3 & 10 & 2 & - & 1 & - & - \\
\hline $\begin{array}{l}\text { Educational/Instructi } \\
\text { onal Materials }\end{array}$ & 3 & - & 1 & 3 & - & 9 & - \\
\hline Book reviews & 6 & 5 & 3 & - & 1 & - & 1 \\
\hline
\end{tabular}

Note. Dashes indicate zero

$N=16$

All experts had at least one of the publications types listed above. Books $(37.50 \%)$, book chapters $(37.50 \%)$, and posters sessions $(62.50 \%)$ were the most popular publications made ranging from one to five times. Educational/Instructional materials were published by 9 (56.25\%) participants from 21 to 25 times. Eight (50.00\%) and five $(31.25 \%)$ experts stated that they published Non-refereed Journals and Proceedings respectively more than 25 times. Two (12.50\%) experts expressed that they had books and books chapters published more than 25 times.

Number of professional presentations and workshops. The professional presentations and workshops that the experts performed were also included in the Professional Profile Characteristics questionnaire. The results are presented in Table 32. 
Table 32. The professional presentations (i.e., how-to, educational/lecture, case study, business, futurecasting, issues, etc.) or workshops done so far by the experts

\begin{tabular}{|l|c|c|c|c|c|c|c|}
\hline Responses & $\mathbf{0}$ & $\mathbf{1 - 5}$ & $\mathbf{6 - 1 0}$ & $\mathbf{1 1}-\mathbf{1 5}$ & $\mathbf{1 6 - 2 0}$ & $\mathbf{2 1 - 2 5}$ & $\begin{array}{c}\text { More } \\
\text { than 25 }\end{array}$ \\
\hline Presentations & - & - & 1 & 1 & - & - & 14 \\
\hline Workshops & - & 2 & 3 & - & - & 1 & 10 \\
\hline
\end{tabular}

Note. Dashes indicate zero

$N=16$

Fourteen $(87.50 \%)$ and ten $(62.50 \%)$ experts conducted more than 25 presentations and workshops respectively. Two (12.50\%) experts presented from six to fifteen times; $5(31.25 \%)$ experts conducted workshops from one to ten times. Only one expert conducted between 21 and 25 workshops.

Number and formats of courses taught. The experts also stated the courses related to HPT which they taught. The question also asked for the modes of the courses, such as in class, on-line, and blended. The results of this question are illustrated in Table 33.

Table 33. The number of courses that the experts taught by course formats

\begin{tabular}{|l|c|c|c|c|c|c|c|}
\hline Responses & $\mathbf{0}$ & $\mathbf{1 - 5}$ & $\mathbf{6 - 1 0}$ & $\mathbf{1 1 - 1 5}$ & $\mathbf{1 6 - 2 0}$ & $\mathbf{2 1 - 2 5}$ & $\begin{array}{c}\text { More } \\
\text { than 25 }\end{array}$ \\
\hline In class & 5 & 6 & - & - & - & - & 5 \\
\hline On-line & 5 & 9 & 1 & 1 & - & - & - \\
\hline Blended & 9 & 4 & 3 & - & - & - & - \\
\hline
\end{tabular}

Note. Dashes indicate zero

$N=16$

Five (31.25\%), five (31.25\%), and nine (56.25\%) participants had never taught courses in class, online, and blended formats in that order. SIX (37.50\%) participants had taught courses in class format between one to five times; nine $(56.25 \%)$ participants had taught online courses from one to five times; four (25.00\%) participants 
had taught blended courses from one to five times. Five (31.25\%) participants had taught in class courses more than 25 times.

Membership to professional organizations. The experts provided their membership to the professional organizations. The results of this are presented in Table 34.

Table 34. The experts' membership to professional organizations

\begin{tabular}{|l|c|c|}
\hline Responses & $\mathbf{n}$ & $\mathbf{\%}$ \\
\hline ISPI - International & 16 & 100.00 \\
\hline ISPI - Local chapter & 7 & 43.75 \\
\hline ASTD - International & 6 & 37.50 \\
\hline ASTD - Local chapter & 2 & 12.50 \\
\hline AECT - International & 3 & 18.75 \\
\hline AECT - Local chapter & - & - \\
\hline No membership & - & - \\
\hline Other & 9 & 56.25 \\
\hline
\end{tabular}

Note. Dashes indicate zero

$\mathrm{N}=16$

All experts were a member of ISPI - International; 6 (37.50\%) participants were members of ASTD - International. The numbers are lower in the local chapter of the same organizations. Seven $(43.75 \%)$ participants were a member of one of the ISPI local chapters; two (12.50\%) participants were a member of one of the ASTD local chapters. Nine (56.25\%) participants indicated membership to "Other" organizations. The most HPT relevant one under "Other" category was ISPI - Europe.

Participation to conferences of professional organizations. The experts were also asked for their participation to the conferences of the professional organizations. The results are illustrated in Table 35. 
Table 35. The number of the participations to the conferences of the experts

\begin{tabular}{|l|c|c|c|c|c|c|c|}
\hline Responses & $\mathbf{0}$ & $\mathbf{1 ~ - ~ 5 ~}$ & $\mathbf{6}$ - 10 & $\mathbf{1 1}$ - 15 & $\mathbf{1 6}$ - 20 & $\mathbf{2 1 ~ - ~ 2 5 ~}$ & $\begin{array}{c}\text { More } \\
\text { than 25 }\end{array}$ \\
\hline $\begin{array}{l}\text { ISPI - The Annual } \\
\text { Performance } \\
\text { Improvement } \\
\text { Conference }\end{array}$ & - & 3 & 2 & 2 & 1 & 3 & 5 \\
\hline $\begin{array}{l}\text { ISPI - The local } \\
\text { chapter } \\
\text { conferences, } \\
\text { seminars, \& } \\
\text { workshops }\end{array}$ & 2 & 3 & 1 & 3 & - & - & 5 \\
\hline $\begin{array}{l}\text { ASTD - Annual } \\
\text { International } \\
\begin{array}{l}\text { Conference \& } \\
\text { Exposition }\end{array}\end{array}$ & 3 & 5 & 5 & 2 & - & - & 1 \\
\hline $\begin{array}{l}\text { ASTD - The local } \\
\text { chapter } \\
\text { conferences, } \\
\text { seminars, \& } \\
\text { workshops }\end{array}$ & 5 & 3 & 1 & 1 & 3 & - & 3 \\
\hline $\begin{array}{l}\text { AECT - Annual } \\
\text { International } \\
\text { Convention }\end{array}$ & 10 & 3 & 1 & - & - & - & 2 \\
\hline $\begin{array}{l}\text { AECT - The local } \\
\text { chapter } \\
\text { conferences, } \\
\text { seminars, \& } \\
\text { workshops }\end{array}$ & 13 & 2 & - & - & - & - & 1 \\
\hline
\end{tabular}

Note. Dashes indicate zero

$\mathrm{N}=16$

Three $(18.75 \%)$ experts had participated in ISPI - The Annual Performance Improvement Conferences one to five times; five (31.25\%) experts had participated in ASTD - Annual International Conference and Expositions between one to five times.

Five (31.25\%) experts stated that they participated in more than 25 ISPI - The Annual Performance Improvement Conference and ISPI - The local chapter conferences, seminars, and workshops. 
Board membership in professional organizations. The experts' board membership was also asked in the questionnaire. The results of this question are presented in Table 36.

Table 36. The experts' board membership in professional organizations

\begin{tabular}{|l|c|c|c|c|c|c|c|}
\hline \multicolumn{1}{|c|}{ Responses } & $\mathbf{1}$ & $\mathbf{2}$ & $\mathbf{3}$ & $\mathbf{4}$ & $\mathbf{5}$ & $\begin{array}{c}\text { More } \\
\text { than } \\
\mathbf{5}\end{array}$ & $\begin{array}{c}\text { Not a } \\
\text { board } \\
\text { member }\end{array}$ \\
\hline $\begin{array}{l}\text { ISPI - } \\
\text { International }\end{array}$ & - & 1 & 1 & 2 & 1 & 3 & 8 \\
\hline $\begin{array}{l}\text { ISPI - Local } \\
\text { chapter }\end{array}$ & - & 3 & 4 & 1 & - & 2 & 6 \\
\hline $\begin{array}{l}\text { ASTD - } \\
\text { International }\end{array}$ & - & - & - & - & - & - & 16 \\
\hline $\begin{array}{l}\text { ASTD - Local } \\
\text { chapter }\end{array}$ & 1 & 1 & - & - & - & 2 & 12 \\
\hline $\begin{array}{l}\text { AECT - } \\
\text { International }\end{array}$ & 1 & - & - & - & - & - & 15 \\
\hline $\begin{array}{l}\text { AECT - Local } \\
\text { chapter }\end{array}$ & - & - & - & - & - & - & 16 \\
\hline
\end{tabular}

Note. Dashes indicate zero

$N=16$

The majority of the participants did not have a board membership in the listed organization. This is an expected result. Three (18.75\%) experts were board members more than five years in ISPI - International; six (37.50\%) participants were a board member more than five years in one of the ISPI local chapters. None of the experts served as a board member in ASTD - International; two (12.50\%) participants served as a board member more than five years in one of the ASTD local chapters.

Volunteering in professional organizations. The experts' volunteer activities were included in the questionnaire. The results of this question are presented in Table 37. 
Table 37. The experts' volunteer activities in the professional organizations

\begin{tabular}{|l|c|c|c|c|c|c|c|}
\hline \multicolumn{1}{|c|}{ Responses } & $\mathbf{1}$ & $\mathbf{2}$ & $\mathbf{3}$ & $\mathbf{4}$ & $\mathbf{5}$ & $\begin{array}{c}\text { More } \\
\text { than } \\
\mathbf{5}\end{array}$ & $\begin{array}{c}\text { Not } \\
\text { Volunteered }\end{array}$ \\
\hline $\begin{array}{l}\text { ISPI - } \\
\text { International }\end{array}$ & - & 1 & - & 2 & - & 11 & 2 \\
\hline $\begin{array}{l}\text { ISPI - Local } \\
\text { chapter }\end{array}$ & - & 1 & - & 3 & - & 6 & 6 \\
\hline $\begin{array}{l}\text { ASTD - } \\
\text { International }\end{array}$ & - & - & - & - & 2 & 2 & 12 \\
\hline $\begin{array}{l}\text { ASTD - Local } \\
\text { chapter }\end{array}$ & - & 1 & - & - & 4 & - & 11 \\
\hline $\begin{array}{l}\text { AECT - } \\
\text { International }\end{array}$ & - & - & 1 & - & - & 2 & 13 \\
\hline $\begin{array}{l}\text { AECT - Local } \\
\text { chapter }\end{array}$ & - & - & - & - & - & - & 16 \\
\hline
\end{tabular}

Note. Dashes indicate zero

$N=16$

Eleven $(68.75 \%)$ experts volunteered more than five times in ISPI - International; six $(37.50 \%)$ experts volunteered more than five times in one of the ISPI local chapters. The majority of the experts ranging from $68.75 \%$ to $100.00 \%$ had neither volunteered for ASTD - International, one of ASTD - Local chapters, AECT - International, nor one of AECT - Local chapters.

Number and types of awards. The experts were asked to provide any awards that they obtained due to their professional activities in the HPT field. Only one expert (6.25\%) expressed that (s)he had not received any awards. The average number of awards per expert was about five. Fifteen (93.75\%) participants stated that they earned awards from ISPI. The awards were: (a) ISPI Life Member Award, (b) Thomas Gilbert Professional Achievement Award, (c) Distinguished Professional Achievement Award, (d) Outstanding Organization Awards, (a) Awards of Excellence for Products, Interventions, and Publications, (b) ISPI Presidential Citation, (c) ISPI Chapter Excellence, (d) ISPI Service Awards, (f) ISPI Local Chapter Awards, (g) and (I) ISPI 
Research Awards. Two experts had also similar awards from different local and international organizations. Some of the most-widely known of these organizations were ASTD, American Educational Research Association (AERA), Association of Educational Communication and Technology (AECT). They had also numerous awards from local and federal government and non-profit organizations.

Job titles. The job titles of the experts were also asked in the questionnaire. The results are presented in Table 38. The initial responses to this question were highly diversified. For this reason, the researcher created categories for the common job titles. Some job titles had very low number of responses. They were grouped as others.

Table 38. The grouped job titles of the experts

\begin{tabular}{|l|c|c|}
\hline \multicolumn{1}{|c|}{ Job Titles } & $\mathbf{n}$ & $\mathbf{\%}$ \\
\hline Academic & 6 & 37.50 \\
\hline Executive & 6 & 37.50 \\
\hline Manager/Supervisor/Director & 2 & 12.50 \\
\hline Consultant & 1 & 6.25 \\
\hline Training \& Development & 1 & 6.25 \\
\hline $\mathrm{N}=16$ &
\end{tabular}

Six $(37.50 \%)$ experts' current job titles were related to academic; another six experts' job titles were under executive category. Two (12.50\%) experts currently work as manager/supervisor/director. One expert expressed his/her current job title as consultant. One of the experts working in academic job also stated that (s)he has a consulting company. Finally, one expert currently works in a job related to training and development.

Age. Age was one of the demographic characteristics of the experts collected with the Professional Profile Characteristics questionnaire. The distributions of the participants' ages are illustrated in Table 39. 
Table 39. The experts' ages

\begin{tabular}{|l|c|c|}
\hline Responses & $\mathbf{n}$ & $\mathbf{\%}$ \\
\hline Less than 30 & - & - \\
\hline $31-40$ & 1 & 6.25 \\
\hline $41-50$ & 1 & 6.25 \\
\hline $51-60$ & 4 & 25.00 \\
\hline $61-70$ & 9 & 56.25 \\
\hline $71-80$ & 1 & 6.25 \\
\hline More than 80 & - & - \\
\hline
\end{tabular}

Note. Dashes indicate zero

$N=16$

The majority of participants $(9,56.25 \%)$ were between 61 and 70 years old. The second most crowded group was 51 to 60 years old with $4(25.00 \%)$ participants. There was only one person in the age ranges of $31-40,41-50$, and $71-80$.

Gender. The second demographic characteristic collected with the Professional Profile Characteristics questionnaire was gender. The results of the participants' gender distribution are presented in Table 40.

Table 40. The experts' gender

\begin{tabular}{|l|c|c|}
\hline Responses & $\mathbf{n}$ & $\mathbf{\%}$ \\
\hline Male & 11 & 68.75 \\
\hline Female & 5 & 31.25 \\
\hline$N=16$
\end{tabular}

Eleven (68.75\%) experts were male, and five (31.25\%) participants were female. The number of male experts was higher than female experts.

Research Question 5: To What Extent Are The Professional Profile Characteristics Of Performance Improvement Practitioners Associated With Their Mental Models Of Expertise Derived From Pathfinder?

Several multiple linear regression analyses were performed to answer this question. The mental model of expertise, generated from relatedness, coherence, and 
similarity, was the dependent variable. The professional profile characteristics are the activities which the experts and participants of the present study completed to date. They were collected to see the relations between the levels of expertise identified based on the mental models of professionals. Thus, the following professional profile characteristics were utilized in the regression analyses as independent variables:

- Years of experience in the field,

- $\%$ of experiences focusing on improving the participants' own performance on their profession, which is self-reported deliberate practice,

- Current industry in which the participants work,

- Number and types of different industries worked previously,

- Number of different organizations worked to date,

- Number of different HPT or HPT related projects completed to date,

- Number of different HPT or HPT related project types completed to date,

- Degrees and number of years spent on each degree,

- Total hours of training related to HPT,

- Number of certificates possessed to date,

- Number and types of publications made to date,

- Number of professional presentations and workshops conducted to date,

- Number of courses taught to date related to HPT,

- Number of memberships to different professional organizations,

- Number of participations to international and regional conferences,

- Years of board memberships in different professional organizations,

- Number of volunteering in different professional organizations, 
- Number of awards obtained to date,

There were also several demographic questions, current job title, age, and gender, under the professional profile characteristics section. They were also included in the analyses as explanatory variables.

Field (2009) suggests that when there are numerous independent predictors to run a multiple regression model, the researcher should run a forced entry model to observe which variables predict the dependent variable significantly and well. Then, these variables are included in the second regression model which should use a stepwise model to reveal the strongest and the most significant predictor(s). The other variables which yielded non-significant and poor prediction magnitude in the first model need to be excluded in the second stepwise model. This technique produces better results for explanatory purposes models. For this study, 258 cases (242 participants and 16 experts) were included in the regression analyses. After case by case investigation, 46 cases were excluded from the analysis due to missing values and outlier issues. All regression models were performed with 212 cases.

In the first forced entry run model, 16 variables did not yield significant results. Only five variables, number of different organizations worked to date, number of different HPT or HPT related projects completed to date, number of different types HPT or HPT related project completed to date, degrees and number of years spent on each degree as well as the total years spend on degrees, and number of courses taught related to HPT to date, predicted the mental model of expertise significantly. In the second stepwise regression model, only these five variables were included in the 
analysis. The following sections will explain details about the second stepwise regression model results.

Table 41 illustrates the correlations between level of expertise and six professional profile characteristics left for the second stepwise regression model.

Table 41. The correlations between Mental Model of Expertise and Six Professional Profile Characteristics

\begin{tabular}{|l|c|c|c|c|c|c|}
\hline & 1 & 2 & 3 & 4 & 5 & 6 \\
\hline 1. Mental Model of Expertise & - & $.254^{* *}$ & $.248^{* *}$ & $-.176^{* *}$ & $.193^{* *}$ & $.185^{* *}$ \\
\hline $\begin{array}{l}\text { 2. Number of different } \\
\text { organizations worked to } \\
\text { date }\end{array}$ & & - & $.283^{* *}$ & -.054 & .152. & $.195^{* *}$ \\
\hline $\begin{array}{l}\text { 3. Number of different HPT or } \\
\text { HPT related projects } \\
\text { completed to date }\end{array}$ & & - & .009 & $.221^{* *}$ & $.181^{* *}$ \\
\hline \begin{tabular}{l} 
4. $\begin{array}{l}\text { Total years spend on } \\
\text { degrees }\end{array}$ \\
\hline $\begin{array}{l}\text { 5. Number of courses taught } \\
\text { related to HPT to date }\end{array}$
\end{tabular} & & & - & $.161^{* *}$ & $.124^{*}$ \\
\hline $\begin{array}{l}\text { 6. Number of different types } \\
\text { of HPT or HPT related } \\
\text { projects complete to date }\end{array}$ & & & & & - & -.038 \\
\hline
\end{tabular}

Note. Dashes indicate one, $N=212$

${ }^{*} p<.05,{ }^{* *} p<.01$

Except for total years spent on degrees, which had a negative correlation $(r=-$ $.176, p<.01)$, the other professional profile characteristics had positive and small correlation ranging from $r=.193, p<.01$ to $r=.254, p<.01$ with level of expertise. Number of different organizations worked to date was associated with number of different HPT or HPT related projects completed to date $(r=.283, p<.01)$ and number of different types of HPT or HPT related projects complete to date $(r=.181, p<.01)$. Moreover, total years spent on degrees were related to number of related HPT courses 
taught to date $(r=.161, p<.01)$ and number of different types of HPT or HPT related projects completed to date $(r=.124, p<.05)$. All of these correlations between professional profile characteristics were small.

The five professional profile characteristics left after the first regression model predicted level of expertise generated from relatedness, coherence, and similarity measures significantly, $F(5,211)=8.745, p<.01$. The details regarding the second stepwise regression model results are illustrated in Table 42.

Table 42. The stepwise regression analysis coefficients results for the mental model of expertise and five professional profile characteristics

\begin{tabular}{|l|c|c|c|}
\hline & $B$ & SE B & $\beta$ \\
\hline Constant & .422 & .103 & $.143^{*}$ \\
\hline $\begin{array}{l}\text { Number of different } \\
\text { organizations worked to date }\end{array}$ & .009 & .004 & $.139^{*}$ \\
\hline $\begin{array}{l}\text { Number of different HPT or } \\
\text { HPT related projects } \\
\text { completed to date }\end{array}$ & .001 & .000 & $-.219^{* *}$ \\
\hline Total years spend on degrees & -.027 & .008 & $.182^{* *}$ \\
\hline $\begin{array}{l}\text { Number of courses taught } \\
\text { related to HPT to date }\end{array}$ & .007 & .003 & $.166^{*}$ \\
\hline $\begin{array}{l}\text { Number of different types of } \\
\text { HPT or HPT related projects } \\
\text { complete to date }\end{array}$ & .032 & .013 & \\
\hline
\end{tabular}

Note. $R=.418, R^{2}=.175$, Adjusted $R^{2}=.155, N=212$

${ }^{*} p<.05,{ }^{* *} p<.01$

The model accounts for $17.5 \%$ of the total variance. The most influential predictor in the model was total years spent on degrees with the highest standardized coefficient value -.219; the least impactful predictor was number of different HPT or HPT related projects completed to date with .139 coefficient value. The result of the regression analysis revealed that the mental model of expertise of HPT professionals is positively 
progressed when they work in or with more organizations, completed more and different varieties of HPT related projects, and taught more HPT related courses regardless of their mode of delivery. Interestingly, the time spent on obtaining degrees had a negative impact on the mental model of expertise. This might be interpreted that the more years education an individual spends, the longer time the individual can progress to an expert's mental model in HPT field.

\section{Summary}

The results of the data analysis are presented in this chapter. Initially, eleven concepts out of 30 were selected based on the responses of 23 experts to the Online Ranking Questionnaire. For the selection, the weighted total scores of each concept were calculated based on the combination of the rank numbers as a primary criterion. Moreover, the total frequencies of each concept were also used as a secondary criterion. These eleven concepts were used to create the Online Concept-Pair Comparison questionnaire which was completed by both the experts and participants. The responses of this questionnaire provided the proximity data utilized for depicting the expert referent model and the common novice model. The expert referent model was found more consistent than the novice common model. Furthermore, the expert referent model was almost $80 \%$ different from the novice common model. Both experts and participants wide range of professional profile characteristics were collected during the research, and they were presented in the results section. Moreover, the proximity data also endorsed the calculation of relatedness, coherence, and similarity measures that were used to create level of expertise. The mental model of expertise scores were used as a dependent variable in the regression analyses. The professional profile 
characteristics were included as independent variables. The results of the regression analyses showed that only six variables predicted the mental model of expertise significantly; however, the other sixteen variables did not predict the mental model of expertise. The variables that predicted the mental model of expertise were number of different organizations worked to date, number of different HPT or HPT related projects completed to date, number of different types HPT or HPT related project completed to date, degrees and number of years spent on each degree as well as the total years spend on degrees, and number of courses taught related to HPT to date. 


\section{CHAPTER 5}

\section{DISCUSSION AND CONCLUSION}

\section{Introduction}

The primary purpose of this study is to reveal performance improvement practitioner expert and novice mental models and identify differences and similarities between these models. The secondary purpose is to analyze the potential association between the professional profile characteristics of performance improvement practitioners and their mental model of expertise regarding the performance improvement field. In the first phase of the study, the online ranking questionnaire was distributed to selected experts to identify the most critical concepts out of 30 concepts for the general understanding of HPT field. Once eleven concepts out of 30 were identified, the same group of experts was contacted to complete the Professional Profile Characteristics and the Online Concept-Comparison questionnaires, which provided proximity data regarding the comparison of each of the eleven concepts to one another. These proximity data were used to create the common expert model, that is, the expert referent model.

In the second phase of the study, the Professional Profile Characteristics and the Online Concept-Comparison questionnaires were distributed to ISPI, ASTD, and AECT Training and Performance Division members. For each participant and expert, Pathfinder derived mental model measures, relatedness, coherence, and similarity, were calculated. These three measures were used to calculate the mental model of expertise. Then, the relationship between the professional profile characteristics and the mental model of expertise were scrutinized. This chapter provides a summary and 
discussion of the research findings as they relate to the study purposes described above, as well as recommendations for further research and potential application by practitioners in the field.

\section{Identification of Concepts}

There were 30 concepts selected for the ranking questionnaire. These concepts were selected after reviewing literature. Majorly, the ISPI standards played a critical role during the selection process since they are usually the first guidance for the professionals who have started a career in the field of HPT.

During the ranking questionnaire, the experts were also asked other concepts that were not included in the list. Some of the experts mentioned the challenging structure of the selection process, which was the main reason for using ranking scale to prioritize things. The online ranking selection task was expected to be a bit challenging. Hence, the researcher aimed to identify the most critical concepts that one needs to know about the field. Moreover, the researcher also attempted to limit the number of concepts because the more number of concepts there are, the more concept-pair items are in the Concept-Pair Comparison questionnaire. For example, when 10 concepts were selected for concept-pair comparisons, there are 45 items. On the other hand, when there are 20 concepts, the number of items increases to 190 items. The number of the items in concept-pair questionnaires increases exponentially. That might decrease the participation rates to the study, which is a critical issue when researchers applied survey techniques and would like to generalize results to the entire population (Johnson \& Christensen, 2008) since this might lead to faulty conclusions and improper inferences (Suter, 2006). 
Villachica, et al. (2001) for instance, applied a similar type of Pathfinder study. They used 30 concepts as well as 435 concept-pairs. 137 participants completed the survey of the study; however, only 73 people completed all concept-pairs. They especially discussed the possible impact of the small sample size on their results. The similar pattern was observed in the present study. 18 experts started the online survey, and 16 experts completed both the Professional Profile Characteristics and the Concept-Pairs Comparison questionnaires. 2 experts did not prefer to complete. The same situation was true for the participants. 335 participants started the survey; 272 completed the Professional Profile Characteristics questionnaire. 242 participants completed both of the Professional Profile Characteristics and the Concept-Pairs Comparison questionnaires. The total number of participants was higher than Villachica, et al.'s study (2001). However, the fewer number of concepts there are, the less opportunity to interpret the models generated there are. For this reason, the models generated in the present study were interpreted by considering one expert's interesting insight for her selection process that "I made my selection based on (1) broad-category items; many of the items are contained within the "categories" and (2) what newcomers to the field and our clients need to know about our field...". Moreover, this statement is also supported because nine of the concepts are explained in ISPI's Performance Technology Standards (ISPI, 2012).

Choosing sufficient number of concepts that do not influence participation rates and the interpretation of the results seem a considerable controversy for Pathfinder studies. Two experts commented that the ranking process might provide an alternative perspective for future researchers. One expert emphasized that "It was challenging to 
choose from a list where all items are important. I chose financial management because financial management includes $\mathrm{ROI}$ and likewise $\mathrm{ROI}$ is part of evaluation. Team development is needed for the change team as well as the other employees who will be participating in the change." Another expert stated a similar comment that "It is a little challenging to prioritize since there is a mix of concepts (such as strategic thinking), established processes (such as performance analysis), and then a variety of intervention categories. It could also be interesting to ask participants to prioritize within these groups, in addition, since it may tell you in greater detail where experts emphasis." According to these two experts' comments, rather than using single concepts, they can group together based on pre-defined characteristics, and then these groups can be selected and utilized in concept-pair comparisons. Thus, the number of concepts can be maintained in reasonable amount; the interpretations of the concepts are also more meaningful. For future studies, it is highly recommended to observe how results vary using groups of similar concepts.

Interestingly, Needs Assessment took twelfth place in the results of concepts ranking. However, Performance Analysis which usually includes the components of needs assessment in several HPT or performance improvement models, e.g., Van Tiem, et al. (2012), Molenda and Pershing (2004), Atkinson and Chalmers (1999), earned the second place. Especially, the HPT model created by Van Tiem, et al. (2012) is a model presented by ISPI in their official web site. In the performance analysis process, the main purpose is to identify performance gaps between what it is and what it should be (Chevalier, 2008). Needs assessment is explained as a systematic process identifying, validating, and prioritizing the discrepancies between current and desired 
status for selecting proper goals and defining right problems (Burton \& Merrill, 1991, Kaufman \& English, 1979). In fact, performance analysis process is a derivate of needs assessment since most of the core concepts and underlying philosophy look very similar. The main distinction between these approaches comes from the different definitions of gap. There are two differences between needs assessment and performance gap analysis (Van Tiem, 2004; Rothwell, 1996b). First, needs assessment concentrates on knowledge, skills, and attitudes; whereas, performance gap analysis focuses on the deficiencies or proficiencies influencing human performance. Second, performance gap analysis intends to the future while needs assessment pay more attention to past and present. Furthermore, performance analysis considers opportunities in addition to needs (Van Tiem, et al., 2004). In this case, the expert might consider this circumstances and choose Performance Analysis rather than Needs Assessment.

One of the eliminated concepts was Intervention Implementation. It was selected by 8 experts (35\%) in one of the ranks; however, its weighted score was low because it was not selected at the higher ranks. When the participants were asked to provide different types of HPT related projects that they have completed, the intervention implementation project types was the lowest percent about $65 \%$. When it is compared to the other projects types, it is about $20 \%$ less than the other project types. For the experts, however, the same situation was not true. There may be reasons for that. First, the professionals are more inclined to work on the projects before implementation stages. Moreover, they might not be preferred by the clients for these kinds of projects. For the experts, especially, well known and who have established themselves in the 
market, the dynamics might totally be reversed compared to the practitioners. Second, there might not be as many opportunities like other project types. Thus, when there is an opportunity, the experts might be the first group of people who they are contacted by the clients. All these reasons might lead the experts did not rank Intervention Implementation at a high level since they might think that it is a concept that the professionals and novices will come across after a while in pursuing their career path.

Another eliminated concept was Instructional Design. One of the experts stated that ". . . I also used a performance technology rather than an instructional technology perspective so I did not include instructional design . .." Instructional design (ID) is accepted as a part of human performance technology (Silber \& Foshay, 2010; Januszewski \& Molenda, 2007). ID is one of numerous solutions offered when there are knowledge and skills deficiencies found after the performance analysis stage. A recent article about the current status, trends, and issues of HPT, which were stemmed from the opinions of a panel of experts, discussed the understanding of performance issues that are currently shifting from ID to more business oriented solutions so that HPT has an opportunity to deal with high leverage issues rather than approaching every issue as lack of knowledge or skills requiring more training or instruction (Pershing, et al., 2008a). For these aforementioned reasons, the experts might not include ID which was one of the critical concepts. Moreover, the same situation might be true for Training included in the same list.

\section{The Expert Model}

The expert model, which was also utilized as the expert referent model for the rest of the study, produced consistent structure. The subsets of this structure can be 
associated with existing models. The concept Performance Analysis is associated with Systems View and Gap Analysis, which is linked to Cause Analysis in the model. Chevalier's (2008) updated performance analysis job aid, which shows a similar structure with aforementioned subset of the expert model, provides an operational combination of performance analysis, performance gaps, system and subsystem components of an organization, and cause analysis. The relationship between Gap Analysis and Cause Analysis seems consistent with Van Tiem's, et al. (2012) HPT model, which is also known ISPI HPT model, and Atkinson and Chalmers (1999) Human Performance Model. In these models, the cause analysis stage is provided by the results of gap analysis.

Pershing (2006) depicted a relationship between Performance Analysis and Systems View. He explains performance analysis stage in his model as four different components: organizational systems, management systems, physical and technical systems, and human and social systems. He suggested that when the performance analysis process is carried out, it starts first with organizational systems, and then it goes to other systems aforementioned order. In fact, Pershing's (2006) performance improvement model utilizes systems view in the performance analysis stage via utilization of action research. Moreover, Rummler (2007) identified performance analysis as the heart of the performance improvement process as well as performance consulting. He suggests that anatomy of performance (AOP) framework should be utilized to understand organizations overarching perspective. According to Rummler (2007), AOP frame has two critical bottom-line tenets: (a) perception of an organization as a complex systems and (b) the impact of interrelatedness of this system's 
components, e.g., alignment and integration, on organization's performance and results. Interestingly, he explained all the processes that he suggests in his book as an application of a consultant's mental model along a case study project. These two different views, one from academia and another one is from the consulting sector, are consistent with the relationship revealed in the present study between Performance Analysis and Systems View in the expert model.

Focus on Results is the center of the expert model. Most of the major models confirm that results must be one of the most critical pieces of performance technology initiatives (ISPI Standards, 2012; Rummler, 2007; Addison \& Haig, 2006; Van Tiem, et al., 2004; Kaufman, et al., 2003). This result is also confirmed by Villachica, et al.'s (2001) empirical study. In their HPT cognitive map, Focus on Results was the center of their model. Focus on Results is connected to Critical Business Issue(s), which is an anchor point for performance consulting and analysis (Rummler, 2007). It creates an invisible linkage between three primary levels of performance and results organizational, process, and job-level. Moreover, it also help performance consultants to identify targets and objectives related to their projects. For this reason, the relationship between Focus on Results and Critical Business Issue(s) was revealed in the expert model.

Another interesting relationship was found between Critical Business Issue(s) and Strategic Thinking. Strategic thinking is a thought process including assessing, envisioning, and creating the future for the people significantly different from the present state (Kuafman, et al., 2003; Heracleous, 2002). Strategic thinking forms strategic planning which is a formal process to identify an organization's desired results from 
operational to societal and long term results and provide a path to achieve these results within a timeframe. Critical business issue(s) can be identified as organizational outputs and are part of the value chain process, which is part of strategic planning, so that professionals in HPT field need to understand strategic thinking well in order to apply and distinguish critical business issue(s) accurately. Therefore, the common expert model produced in this study included that type of relationship. The experts believed that Strategic Thinking needs to be in place when Critical Business Issue(s) are considered.

Measurement and Evaluation concept was another concept linked to Focus on Results. Moreover, it was also the linkage of Systematic Approach, Intervention Selection, Design, and Development, and Establishing Partnership with Client concepts to Focus on Results. The experts thought that these three concepts are not meaningful without Measurement and Evaluation emphasizing its criticality once again. In general, evaluation is defined as "the identification, clarification, and application of defensible criteria to determine an evaluation object's value (worth or merit) in relationship to those criteria" (Fitzpatrick, Sanders \& Worthen, 2004, p. 5); evaluation includes inquiry and judgment methods, such as determining standards for judging quality, deciding whether these judging qualities are relative or absolute, collecting relevant information, applying the standards to make a meaningful conclusion, make recommendations based on the conclusion to optimize the evaluation object to its planned purposes, and help stakeholders make decisions about acceptance, continuation, or development. When we narrow the broad evaluation term to performance improvement, the relationship between Measurement and Evaluation and the other three concepts might be 
understood better. First, it is a mediator between results and interventions selected, designed, or developed to impact those results. The results provide a framework for the judging standards; the intervention is an evaluation object. Systematic Approach ensures standardized judgment quality and procedures so that these procedures can be replicable in other contexts. When these standards are established, there are some conclusions that will help stakeholders, who are usually clients in performance improvement projects, make the right decision of the intervention or identified performance gaps or issues. At this time, measurement and evaluation and its linkage to focusing on results promote establishing partnerships with clients.

The current global evaluation trends explained by Phillips and Phillips (2007) might provide an underlying reason for the relations aforementioned about Measurement and Evaluation in the expert model. Some of the key trends are clients' driven needs increasing interest to evaluation, shift from reactive to proactive approach that addresses evaluation as early as possible in the project cycle, systematic and methodological evaluation processes, organizations' increasing interest to evaluation, etc. Since mental models are very flexible and open to change, these trends might have substantial impact on the approach the professionals use to organize evaluation in their current knowledge structures.

Even though the experts stressed the relationship between Measurement and Evaluation and Focus on Results, the real practices of performance improvement practitioners were not consistent with it (Guerra-Lopez \& Leigh, 2009; Schaffer \& Keller, 2003). While Schaffer and Keller (2003) identified that the professionals utilized resultoriented, that is, organizational level effective performance improvement and measuring 
systems and processes at low level, Guerra-Lopez and Leigh (2009) found that the measurement and evaluation did not seem to be the central components for performance improvement projects. Moreover, some expert panelists who participated in Pershing, Lee, and Cheng's (2008b) study regarding the current status, trends, and issues of HPT stated that there is a lack of effort in the areas of business impact measurement, assessment, and evaluation. The initiatives to make solid connection between the beliefs of experts regarding evaluation and the real applications would be a valuable contribution for the future of the field.

\section{The Expert vs. Novice Model}

As previously stated in other expert and novice comparison studies (Adelson, 1981; Boshuizen \& Schmidt, 1992; Burkhardt, et al., 1997; Chi, Feltovich, et al., 1981; Chi, Glaser, et al. 1982; Chi \& Koeske, 1983; Ericsson, et al., 2007a; Feltovich, et al., 1984; Hill, 2006; Hmelo-Silver, et al., 2007; Le Maistre, 1998; McKeithen, et al., 1981, Mitchell \& Unsworth, 2005; Perez \& Emery 1995; Perez, Fleming, et al., 1995; Randel, et al., 1996; Villachica, et al., 2001), the common mental model of experts differed from the common mental model of novices of the professionals who participated in the present study.

The common expert model demonstrated a more coherent structure than the common novice model. This was also confirmed with their coherence measures, which were more than .4 and less than .2 respectively, derived from Pathfinder analysis. There are numerous studies consistent with this finding in the present study. Chi and Koeske (1983) found that the structure of knowledge and information acquired by a person at a high knowledge level differs from the structure of those who had low knowledge level. 
The high knowledge level person's structure demonstrated more cohesiveness and interrelatedness. Hmelo-Silver, et al. (2007) pointed out that novices are more capable of understanding a system's elements; however, experts surpass novices in terms of understanding the interrelatedness of the systems' elements and their role to reach the common goal. This difference occurs due to experts' utilization of more consistent schemas. Another consistent study by Burkhardt, et al. (1997) in the computer programming domain indicated that expertise had a significant impact on tasks that require utilizing and creating a mental model; moreover, the mental models related to this task built by experts were stronger than those built by novices. A study comparing the cognitive map novice and expert HPT professionals (Villachica, et al., 2001) indicated that there were significant differences between expert and novice performance improvement professionals when their relatedness and similarities were scrutinized. Le Maistre (1998) found that there are prominent differences between an expert and novice instructional designers' design characteristics including well-organized instructional design knowledgebase while they were performing the same task. The present study confirmed that the expert mental model of HPT professionals demonstrated more coherent and consistent structure than the novice mental models.

The common novice model had a more linear structure compared to the common expert model. Adelson (1981) found that novices usually utilized syntax-based organization of knowledge which is straightforward and step by step structure in a computer programming task. Moreover, novices focused on superficial and surface characteristics of problems compared to the experts who focused on deep structures and underlying principles or theories behind the components and their relations of the 
same problem (Chi, et al., 1981; Lister, et al., 2006; Rowland, 1992; Schoenfeld \& Herrmann, 1982). These differences between expert and novice approaches to understanding the problem also contend the less complexity of novice mental models. Moreover, Perez, et al. (1995) indicated that one of the major distinctions between novice and expert instructional designers during a design task was the structure of their understanding related to the design problem. Novices had fewer linkages between entities of the problem compared to the experts. Fiske, et al. (1983) investigated the expert and novice differences in political sciences. Novices were more likely to focus on consistent information within a political situation. Hence, consistent information is clearly defined and associated with its surroundings. Moreover, the consequences of consistent information are standard; therefore, novices might not need to utilize more complex and deeply organized knowledge.

\section{Relationship between Professional Activities and Mental Model of Expertise}

The mental model of expertise generated based on the total of three measures derived from Pathfinder analysis were positively influenced by the number of the organizations worked, the number and varieties of the projects completed, and the HPT related courses that have been taught so far. HPT inherently values comprehensive and systemic ideas and solutions (Pershing, 2006). Every organization is a system including sub-systems and interacting with a supra-system and other systems. Subsystems interact and are interrelated to each other to achieve common goals. There are numerous and complex rules, principles, policies, etc. making the whole organization progress; there are also boundaries separating an organization from other organizations. Working for or with different organizations requires an understanding of a 
complex system from the beginning. Each organization is a unique case to investigate; therefore, they add enriched experiences to the professionals' background. For this reason, the numbers of organizations may help HPT professionals to develop more consistent and well organized mental models for the general understanding of the field. The numbers and diverse variety of projects provide opportunities for applying systematic procedures of the field. Consequently, they either purposefully or accidentally form situations nurturing deliberate practices (Ericsson \& Charness, 1994) or deliberate performances (Fadde \& Klein, 2010) leading to well organized knowledge or mental models that are discussed as one of the indicators of expertise. Two studies from ID literature demonstrated that ID experts utilized their background knowledge and skills related to subject matter and previous experiences regarding the problem that they were trying to provide solutions (Ertmer, et al., 2008; Hardré, Ge \& Thomas, 2006). Since the background and previous experiences were not explained in detail in these studies, they might be influenced by working for or with different organizations and completing numerous and diverse projects as well as other factors. The numbers of courses taught regardless of their modes were the highest professional profile characteristics associated with the mental model of expertise. Instructors usually build a guidance model of the course content to demonstrate all possible relationships between the key concepts, and they apply different approaches, strategies, methods, and techniques to shape students' mental models or schemas. For this reason, instructors' mental models related to the topic taught are utilized as referent or criteria models to measure learning and performance, e.g., Acton, et al. (1994), DiCerbo (2007), Goldsmith, et al. (1991), Kahler (2003), McGaghie, et al. (2000), Schlomske and Pirnay- 
Dummer (2009). This would be a conceivable reason to explain the positive relationship of the HPT related courses taught with the mental model of expertise.

The total years spent on degree(s) had a negative relationship with the mental model of expertise. When four other professional profile characteristics are considered, attending schools and obtaining any degree looked less practical. The education institutions might embed practical applications (e.g. internship programs) to have their students get involved in more real-life performance improvement settings for a semester or a year; however, this cannot be the same experience as increasing experience in the field as an active professional. That might be one reason for this reverse direction relationship. Moreover, when people completed a course including several models or techniques, they usually have high motivation to apply them into the real-settings. This might occur as an attempt to apply every step that they learned in the models or techniques straightforwardly. In this case, they tend to neglect most of the contextual factors. These groups of behaviors and cognitive components triggering these behaviors are usually observed at novices in several domains (Adelson, 1981; Chi, et al., 1981; Fiske, et al., 1983; Lister, et al., 2006; Perez, et al., 1995; Schoenfeld \& Herrmann, 1982). For more specific example, Le Maistre (1998) compared two instructional designers and provided also how these designers were educated about ID. One had 15 years' experience in ID, did not have any advanced degree, and was taught by a mentor; the other one had five years' experience and had master's degree in which (s)he took several ID courses. The second designer focused on more surface and cosmetic revisions related to the task which they were asked to complete. The education level regarding the domain did not show an impact in this study. More studies 
are needed to draw solid conclusions about the years spent on education and the mental model of expertise in HPT.

Moreover, in order to understand the relationship between expertise in HPT and educational degrees more obviously, the domains of degrees need further analysis. Witucki (2006) revealed that novices acquired their intervention knowledge via formal education; whereas, the experts acquired theirs by informal education. The current diversity of these education levels might be another reason for the negative relationship. In the present study, since the domains of degrees were not included in professional profile characteristics, no evidence can be produced to make detailed discussion of the potential impact of different domains of degree(s) on the mental model of expertise. Studies that investigate this relationship would provide a deeper insight regarding the issue.

The number of publications was not significantly associated with the mental model of expertise of the professionals. Villachica, et al. (2001) found that the number of books published was related to the coherence of the cognitive maps of HPT professionals. They discussed plausible reasons which were specific to the context of the study. First, authors devote enormous amount of time to build a clear framework for their books focusing on a type of audience, and this improves authors' cognitive schemas considerably. Second, their data collection process for generating cognitive maps was tedious and needed a long time. Since book authors were used to spending a lot of time while writing books, they may persist to complete the questionnaires. The current study utilized less number of concepts to generate the experts and professionals' mental models. Therefore, the potential impacts of previously developed 
complex mental models and task-persistence would not show the similar impact. Moreover, this study aimed to identify mental models of professionals about the general understanding of the field. This general nature might not be as complex as Villachica, et al.'s model (2001) to reveal any significant relationship. This is another area requiring more research in order to infer strong associations and causal-mechanisms.

The present study did not reveal any significant relationship between years of experiences in the field and the mental model of expertise. Van Tiem (2004) indicated that there was a significant and positive correlation between the years of experience and the intervention usage as well as the level of expertise. The more years a professional spend in the field, the more knowledge of the interventions the professional has. One reason for these contradicting results is the difference between the definition of the expertise. In the present study, the expertise was identified based on several criteria, such as more than ten years of experience, publications, active membership in professional organizations, and another expert's referral. Van Tiem (2006) utilized the personal reporting of the study participants to identify the level of expertise. In the present study, the mental models of the HPT professionals based on eleven general concepts was the primary focus to observe the relations with previously completed professional activities. Van Tiem (2006) focused on the usage of interventions that is more related to practical application; whereas, mental models are organization of knowledge and skills that endorse these practical applications. However, Witucki (2006) found that the years of experience and the way to acquire the specific HPT knowledge about interventions did not have much influence in the usage of the interventions. He utilized Ericsson and Charness's (1994) ten years rule for identifying experts, which was 
one criterion in the present study. Moreover, Ericsson and Charness (1994) states that years of experience is not a good factor to explain expertise in most cases because it does not completely cover deliberate practices becoming an expert in a domain. Another evidence from an empirical study contended that the selection of experts based on performance criteria rather than years of experience resulted in well-structured mental model when they were troubleshooting some technical problems (Bradley, et al., 2006). Years of experience might be a broad factor to investigate its relationship to expertise as well as the components of expertise. The more specific professional activities completed during the time frame of years of experience may lead enriched interpretable results with not only HPT but also all other domains of expertise.

The present study attempted to measure deliberate practice to differentiate it from years of experience. The experts and professionals were asked to identify the percent of their years of experience accounting for their deliberate practices about HPT. The definition of deliberate practice was also provided in the question. However, deliberate practice did not yield any significant relationship like years of experience. This result was obtained due to the problematic approach to measure deliberate practice. Ericsson and Charness (1994) suggests that deliberate practice needs to be identified in details via prolonged self-reporting or observations. This helps researchers to quantify the accurate amount of time spent on improving specific skills associated with expertise in a domain. They do not suggest using merely self-reporting without any other supporting measures. The present study utilized an online survey to reach the experts and professionals so that it was not feasible to collect any other supporting measures which Ericsson and Charness (1994) suggested. However, Fadde and Klein 
(2010) discussed that deliberate practices are usually more appropriate for psychomotor skills, e.g., playing an instrument, and cannot be easily identified in the jobs requiring knowledge work which all business and professional work are based; therefore, deliberate practice is impractical for business people. They offered deliberate performance, which is thought as the part of routine jobs and just-in training activity, instead of deliberate practice. They also offered a four-component model that fosters deliberate performance. These components are estimation, experimentation, extrapolation, and explanation. Future qualitative studies using smaller sample sizes can be performed to observe the relationship between deliberate practices or deliberate performances considering the four-components and level of expertise of HPT professionals.

Finally, the total variance obtained for this result was not high. This is expected since there are numerous factors which researchers cannot control especially in survey research. Variance can be increased by utilizing highly associated factors with the mental model expertise. In order to find out these kinds of variables, prolonged observation of individuals' performances, which was also performed by Ericsson and Charness (1994), might be a good technique for further studies. However, there are two very critical issues when researchers will use different and numerous variables. First, the more numbers of variables may increase variance; however, the stability of the model might be hindered. For this reason, they need to check critical parameters of the model to observe the best-fit. Assumptions of regression analysis must be taken into consideration for this. Second, when there are numerous variables, there is a high 
possibility of having variables greatly associated with each other. That causes inflated results from regression analysis as well as unreliable interpretations.

\section{Demographic Characteristics and Mental Model of Expertise}

The demographic variables of age, gender, and job title were collected in the present study. None of these variables were associated with the mental model of expertise. Age is a controversial demographic character in expertise research. For instance, Charness, et al. (1996) found that age had negative impact on chess players' chess skills in international tournaments. However, Day and Lord (1992) indicated that older experts demonstrated better performance than younger experts in terms of classifications of the organizational problems.

Krampe and Charness (2006) argued that since general cognitive abilities which usually do not include skills, contextual factors, everyday competencies, real-life expertise, etc., the inconclusive association between age and expertise at older ages cannot be sufficient to draw solid conclusions. For this reason, more ecological approaches to investigate this relationship emerged.

\section{Limitations of the Study}

Sampling strategies was one of the potential limitations, of the present study, especially for generalizability. Both purposive and convenience sampling strategies cannot be said to be representative of the population since they are not random sampling techniques. In fact, the vast majority of educational researchers cannot use random sampling due to time, money and resource constraints (Wallen \& Fraenkel, 2001). On the other hand, purposive and convenience sampling strategies are quite feasible. 
In the present study, an online survey technique was utilized as the main data collection procedure. The major obstacles of online surveys are sampling issues (Wright, 2005). Online internet surveys reach a group of people where characteristics cannot be defined clearly; in addition, the non-response rate is not easily identified or forecasted. HPT practitioners currently might not show consistent population characteristics because the majority of professionals are originally from different backgrounds or fields. For example, expert professionals improved their knowledge related to interventions via informal education; novice professionals had the same types of knowledge from formal education (Witucki, 2006). Thus, any other data collection technique utilized in HPT research may have the same issues. Second, participation in this study was voluntary, Voluntary participation is based on self-selection, which is another constraint of online surveys (Thomson, Surface, Martin \& Sanders, 2003). There may be some individuals who are more willing to complete online surveys, and there are some other people who ignore the invitation to participate. In addition to selfselection, there is no possibility to differentiate the participants who have responded to the survey and who do not respond since any demographic or other critical information about non-respondents remain unknown (Guerra, 2003). Due to self-selection and lack of information about non-respondents, the researchers cannot be sure whether the study sample is inherently biased or not (Leigh \& Tracey, 2010). All of the limitations discussed in this section so far prevent researchers from making proper generalizations and estimating the findings to population. In order to take these limitations under control, the researcher attempted to reach three different professional organizations first and then social networking sites second since the return-rate was low initially. 
Specifically, AECT and ASTD members did not show much interest to participate in this study. Finally, the researcher used the personal contacts via e-mails or previously collected business cards. According to Wright (2005), trying to reach out different groups for data collection may provide multiple applications of the survey or replication that is recommended as the only way to see a reliable picture of online survey participants.

Low return-rate of online surveys was another limitation. It influenced the number of participants as well as the sample size of the study that causes some issues about generalization. Sending reminder messages are the common technique to increase the return-rate. In the proposed study, this technique was utilized; however, since the return-rate was low to conduct a rigorous analysis at the beginning of the data collection, the researcher took advantage of additional techniques which are positively associated with high return-rate. For instance, Cook, Heath, \& Thompson (2000) indicated that the number of contacts, personal contact, and pre-contacts were the factors increasing return-rate in web-based surveys in a meta-analysis study in which they analyzed 49 studies and 69 survey questionnaires. Similar to Cook, et al. (2000) results, Sheehan (2001) presented that the year of the survey implemented and the number of follow-up contacted had a significant impact on high return-rate of e-mail surveys.

Another limitation was the self-report nature of the data that the researcher collected. The researcher assumed that self-report data represent the actual condition of participants' feelings and opinions. Since the self-report data must be kept anonymous and confidential in surveys, it was not possible to verify the results 
separately (Witucki, 2006). However, individuals' mental models, which are the major focus of this study, are specific to the person's subjective perception since mental models contain structure of knowledge regarding the individual's insight about the world (Winn, 2004). The self-report surveys are more proper if the researcher concentrates on the individual's personal experiences and opinions ("Self-Report Method.," 2008).

The final limitation of the present study was the number of concepts used to generate the experts and professionals' mental model. Since 11 concepts, selected by the experts via using a ranking scale, were used, the mental model and all related measures generated from these models were limited to 11 concepts and their represented meaning.

\section{Implications for the Field of Performance Technology}

Since the current study focused on the mental model of HPT expertise for the field's general understanding and related professional practices associated with this mental model, there are several areas that the current study may enlighten. The first one of these areas is development of HPT expertise. There are either informal or formal ways to obtain HPT expertise. The common expert model about the general understanding of HPT revealed in this study may be used as a guide to develop novice performance improvement professionals' mental models and help novices approach the expert mental model. The current study may inform performance improvement experts mentoring new practitioners who have recently started their HPT career. They can utilize the common mental model of experts to start an initial inquiry to understand the field in more detailed manner. As also suggested in Villachica, et al. (2001), the common mental model may be utilized as a mind tool to guide expert performance 
improvement practitioners while they are mentoring new practitioners. The situation depicted above about mentoring is not uncommon since new practitioners usually need additional preparation before starting their career in the market. If they start as an internal practitioner, it takes time to understand their organization and its current dynamics. Most of time, other colleagues in the same department are good sources for the preparation. If they start as external practitioners (e.g. performance consultant), it is always wise to talk with other experienced consultants.

In terms of a formal way to acquire HPT expertise, the colleges, universities, and institutions providing HPT related training or workshops would be the most proper organizations to be informed with the current study results. The current study has potential implications at either course design or curriculum levels. At the course design level, the common mental model of experts may be utilized as advance organizers to introduce students to the big picture of the field at a first glance. One of the expected outcomes in instruction and learning processes is improved mental models of students regarding the course content. Ertmer, et al. (2009) found that explicit guidance based on expert thinking might help novice instructional designers focusing on critical aspects of a design problem situation. The common mental model of experts may be used as a guidance to improve students' mental models. However, it should be noted that mental models of individuals are not constant and fully complete. For this reason, they need to be updated on a regular basis. Pathfinder technique used in this study may provide an approach for this. Finally, the common mental model of experts also can be utilized as a measurement tool to establish bottom-lines for evaluation. 
The professional activities associated with the mental model expertise may provide additional information for the development and improvement of curriculum in HPT programs. The number of organizations and the number and different types of projects and activities are vastly real-life based practices. The HPT curriculum in the institutions may focus on providing more vicarious experiences. Ertmer, et al. (2008) suggested that vicarious experiences might be provided in the current graduate programs by incorporating "use of case studies; internship and practicum experiences; guest speakers; as well as consulting with, and working for, real clients as part of a studio design approach." (p. 35). Another professional activity associated with the mental model of expertise was the teaching of courses. In the light of this result, the HPT programs may provide opportunities to teach or co-teach one or couple introductory level classes as a part of students' degree requirements. Students may teach either alone or as a part of student teams.

Currently, several professional organizations provide diverse certifications related to HPT, such as ISPI's Certified Performance Technologist and ASTD's Certified Professionals in Learning and Performance. These processes for credentialing are very extensive, and professional organizations are pressured to demonstrate whether their certifications are both vigorous and convincing in the current market. The results from this study may inform existing certification processes. First, the assessment criteria of certifications may be updated. Usually, the certification processes review previous experiences and professional activities completed to date in the field. If the most critical experiences and practices are clearly identified based on more expertise studies like the present one, experiences and practices may be easily categorized depending on their 
impact on HPT expertise. This improves the credibility and validity of the certifications as well. Moreover, focusing on the most critical experiences and practices may decrease large amounts of efforts for certification review processes. Second, some certifications (e.g. CPLP) need knowledge testing in addition to evidence of experience and previous work. The present study may provide an alternative or additional assessment technique to knowledge tests. The common mental model of experts revealed in this study or its updated versions can be proper criteria to measure and evaluate the professionals' knowledge level.

There are numerous efforts to portray the required competencies for performance improvement professionals, e.g., Chow (2010), Fox and Klein (2003), Giberson (2010), Guerra (2003), Lauer (2008), Vadivelu and Klein (2008). Competency is "a cluster of related knowledge, skills and attitudes $(K, S, A)$ that affects a major part of one's job (a role or responsibility), that correlates with performance on the job, that can be measured against well-accepted standards, and that can be improved via training and development." (Parry, 1996, p. 50). As also noted by Villachica, et al. (2001), common expert models may assist development of existing HPT competencies or creation of new competencies. The common mental model of experts may demonstrate what knowledge of experts is related to a competency and how this knowledge is organized. Moreover, professional practices associated with the mental models of experts might have the potential to explain dynamics between competencies and related behaviors leading to outputs and results (Parry, 1996). Expertise based competency models may be developed.

\section{Recommendations for Further Research}


The first recommendation for further studies is the replication of the present study with different sample characteristics and sizes. One group of studies may utilize different sampling techniques so they may have different sample characteristics. They may also have an opportunity to estimate the findings to the overall population if random sampling techniques are utilized. Another group of studies may try to reach more participants by using the same professional profile characteristics and criteria for selecting experts of the current study by including other professional organizations.

The current study utilized one type of expert study approaches and knowledge elicitation techniques. There are other numerous methods for studying expertise, e.g., multiple-case studies, single case studies, focus-groups, true or quasi experimental, etc., and other different techniques to elicit experts' knowledge organizations , e.g., card-sorting, think-aloud, cognitive-task analysis, etc. The future studies related to HPT expertise which utilizes these different research methods and knowledge elicitation techniques would contribute to the development of the field.

The present study utilized stand-alone concepts for the ranking of the most critical concepts. Two experts suggested that the stand-alone concepts can be grouped together based on their commonalities, and then these groups can be used to create grouped-concept pair comparisons. As a result, the number of the pairs can be in reasonable number, and the interpretations of the mental models generated from these grouped concepts are also more meaningful and overarching. The future studies using groups of similar concepts are highly valued to observe how the results vary from the present study. 
The concept Measurement and Evaluation was valued by the experts and professionals in the present study. Yet, there are discrepancies between how much the experts and professionals value it and real life applications (Guerra-Lopez \& Leigh, 2009; Pershing, et al., 2008b; Schaffer \& Keller, 2003). For this reason, not only investigation of these discrepancies but also creation of useful implications to close these discrepancies may add values to the current status of the field. The studies to establish solid connection between the beliefs of experts regarding evaluation and real life applications would result in critical contributions to the field as well.

More studies are needed to investigate the relationship between years spent on attaining degrees and expertise in HPT. Hence, the current status of research on this issue is not very much conclusive. There are two dimensions taken into consideration for studying this relationship. The first one is the types of the degrees, e.g., Master, Educational Specialist, Doctor of Philosophy, Doctorate of Education, and so forth,, and their individual impact on expertise; the second one is the domain of the degrees and the differences between the domains in terms of developing HPT expertise. Further studies may utilize these dimensions either as stand-alone or combined. Well-designed studies investigating the combination of these dimensions might provide enhanced interpretations.

The number of publications was not associated with mental model of expertise for the general understanding of HPT. For this reason, there cannot be much convincing findings and discussion presented in the present study. Because of few numbers of studies to enlighten the relationship of publications and HPT expertise, further studies need to be conducted. Since publications are especially more appreciated for 
promotions in academia, the specific studies focusing on this industry may be one approach for further studies. Moreover, including other industries and comparing the impact of publications on expertise among different industries may be an alternative approach.

Years of experience may be a very general reason to explain expertise, especially, for the fields, like HPT, where knowledge workers are key players (Fadde \& Klein, 2010). Years of experience need to be broken into more specific and detailed professional activities completed during that time frame. The studies using years of experience may produce invaluable consequences with not only HPT but also all other domains of expertise.

The only empirical evidence that explains expertise, deliberate practice, was not associated with the mental model of expertise about the general understanding of the HPT. The definition of Ericsson and Charness's (1994) deliberate practice is not very suitable to the fields like HPT since most of the professional activities are based on knowledge work, and deliberate practice is not meaningful to the current business people (Fadde \& Klein, 2010). In the light of this discussion, further studies may identify the most suitable practices for developing HPT expertise and their impact on HPT expertise.

Krampe and Charness (2006) state that the current research on expertise demonstrates that older adults can maintain their expertise if they keep their deliberate practice regarding the expertise domain at least up to age 70 s. Moreover, the older workers' characteristics explained in Moseley and Dessinger (2007) may be taken into consideration along with investigating how they maintain their expertise at later ages. 
Since, in the present study, more than $50 \%$ of the professionals and experts were found in the older worker and learner category on the basis of Moseley and Dessinger's classification (2007), future studies providing invaluable implications regarding this issue would be highly appreciated in the field.

There were also numerous professional profile characteristics that did not yield any significant relationship with the mental models of experts and professionals in this study. Each of them would establish a baseline for separate both quantitative and qualitative future studies on HPT expertise; moreover, they would be utilized as different combinations. Instead of directly affecting the experts' superior performance, they might influence motivation of novices to lead them to superior performance (Butterworth, 2006). Those types of indirect or mediated relations may provide more complex models of the relationship between factors and expertise or its components in the field.

\section{Conclusion}

In this section, the results of the current study were discussed. Several major points were emphasized, such as the possible reasons for eliminated concepts, the literature connection of the mental model of expertise, the underlying points for the difference between the mental models of experts and novices, and the relations between the professional activities and the mental model of expertise. The limitations of the study were explained in detail. Along with the results, the possible implications of the study on performance technology and further studies for scholars in the field were recommended.

Finally, the present study was conducted to make a contribution to the recently released research trends for HPT by Huglin, et al. (2007). The main purpose of the 
present study was to make a contribution to one of these trends, the comparison of experts' and novices' mental models and practices. Of course, one study is not sufficient to fulfill the needs stemmed from these trends. Further studies were already expressed in the previous section related to the topic of the present study. On the other hand, the field needs more research to establish more solid foundations and practices. In addition to the topic of the current study, other trends (Huglin, et al., 2007; Pershing, et al., 2008b), which are (a) operational definitions of key research variables, (b) measuring added-value, (c) identification of best practices for optimizing interventions, (d) the added values of HPT's and other fields, (e) creation of an integrated framework for the existing research, would be an excellent basis for preliminary ideas and guidance for future scholars. 


\author{
and Amendments
}

\author{
Notice of Expedited Approval
}

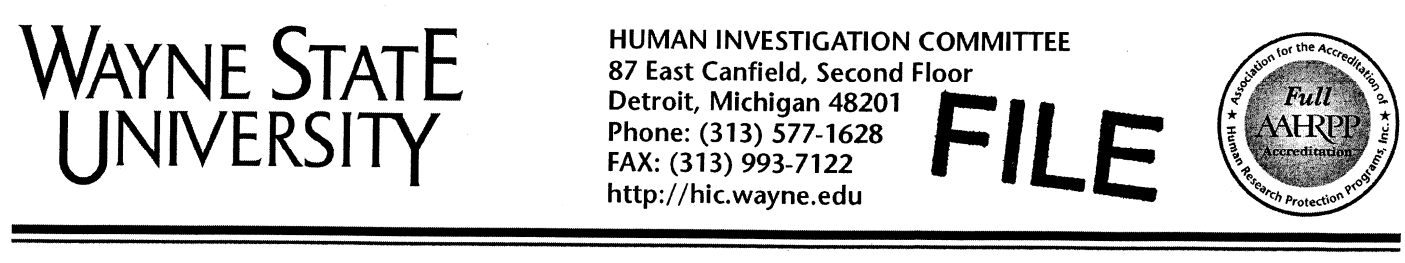

\title{
NOTICE OF EXPEDITED APPROVAL
}

To: Sacip Toker

Administration \& Organization Stud

1261 Kirts Blvd.

From: Dr. Scott Millis

Date: April 06, 2011

RE: HIC \#: 036211B3E

Protocol Title: The Mental Model Comparison of Expert and Novice Performance Improvement Practitioners Funding Source:

Protocol \#: $\quad 1103009531$

Expiration Date: April 05, 2012

Risk Level / Category: Research not involving greater than minimal risk

The above-referenced protocol and items listed below (if applicable) were APPROVED following Expedited Review Category (\#7 )* by the Chairperson/designee for the Wayne State University Institutional Review Board (B3) for the period of 04/06/2011 through 04/05/2012. This approval does not replace any departmental or other approvals that may be required.

- Protocol Summary Form, revised 3-31-11.

- Receipt of a research protocol

- Cover Letter to Experts, dated 4-5-11.

- Research Information Sheet (Experts) dated 4-5-11.

- Cover Letter to Professionals, dated 4-5-11.

- Research Information Sheet (Participants) dated 4-5-11.

- NOTE TO PI: Please submit an amendment providing the revised e-mail cover letters with the production link when data collection is ready to begin.

- Federal regulations require that all research be reviewed at least annually. You may receive a "Continuation Renewal Reminder" approximately two months prior to the expiration date; however, it is the Principal Investigator's responsibility to obtain review and continued approval before the expiration date. Data collected during a period of lapsed approval is unapproved research and can never be reported or published as research data.

- All changes or amendments to the above-referenced protocol require review and approval by the HIC BEFORE implementation.

- Adverse Reactions/Unexpected Events (AR/UE) must be submitted on the appropriate form within the timeframe specified in the HIC Policy (http://www.hic.wayne.edu/hicpol.html)

NOTE:

1. Upon notification of an impending regulatory site visit, hold notification, and/or external audit the HIC office must be contacted immediately

2. Forms should be downloaded from the HIC website at each use.

*Based on the Expedited Review List, revised November 1998 
Notice of Expedited Amendment Approval - June 02, 2011

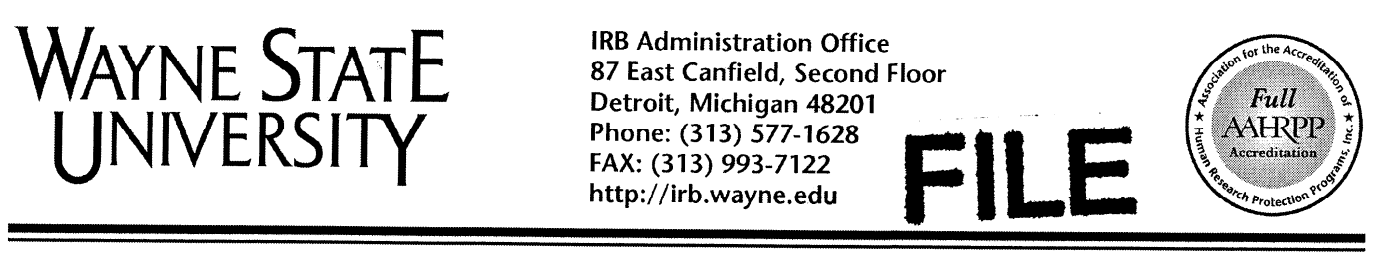

NOTICE OF EXPEDITED AMENDMENT APPROVAL

To: Sacip Toker

Administration \& Organization Stud

for 1261 Kirts Blvd.

From: Dr. Scott Millis

Chairperson, Behavioral Institutional Review Board (B3)

Date: June 02, 2011

RE: IRB \#: 036211B3E

Protocol Title: The Mental Model Comparison of Expert and Novice Performance Improvement Practitioners

Funding Source:

Protocol \#: $\quad 1103009531$

Expiration Date: $\quad$ April 05, 2012

Risk Level / Category: Research not involving greater than minimal risk

The above-referenced protocol amendment, as itemized below, was reviewed by the Chairperson/designee of the Wayne State University Institutional Review Board (B3) and is APPROVED effective immediately.

- Protocol- Data collection methods and/or instruments modified to reflect changes in questionnaires. The structure of the ranking questionnaire was changed, participants were asked to volunteer their e-mail addresses for contact, and participants were asked to voluntarily provide other experts' name and e-mail addresses. The second questionnaire was revised for clarification and updated instructions.

- Cover Letter for HPT Professionals (dated 05/31/2011)- Invitation to Participate in Research Study updated to include the production link of each online survey and to add a 150-word version of the cover letter.

- Cover Letter for HPT Experts (dated 05/31/2011)- Invitation to Participate in Research Study updated to include the production link of each online survey.

- Information Sheet (dated 05/31/2011)- Research Information Sheet (Participants) and Research Information Sheet (Experts) both revised for better clarity. 


\section{Notice of Expedited Amendment Approval - July 01, 2011}

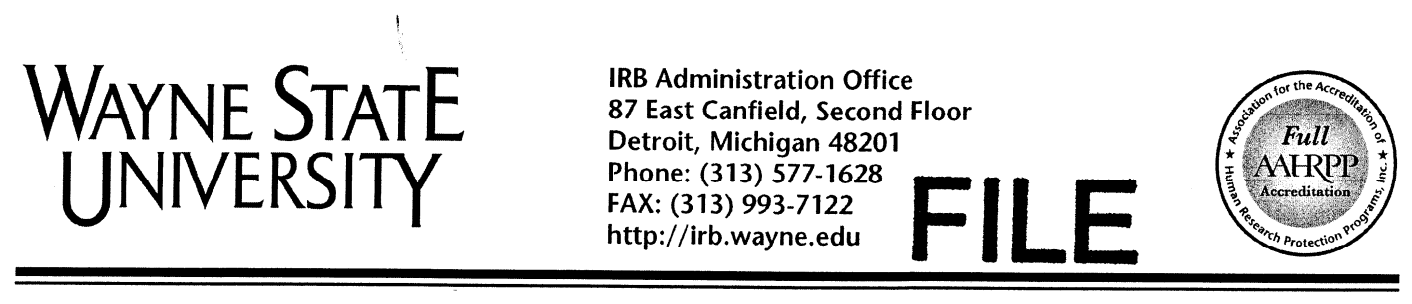

NOTICE OF EXPEDITED AMENDMENT APPROVAL

To: Sacip Toker

Administration \& Organization Stud

1261 Kirts Blvd.

From: Dr. Scott Millis

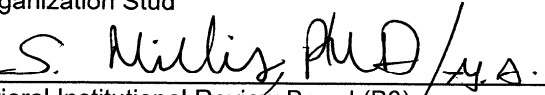

Chairperson, Behavioral Institutional Review Board (B3)

Date: July 01, 2011

RE: IRB \#:

036211B3E

Protocol Title: The Mental Model Comparison of Expert and Novice Performance Improvement Practitioners

Funding Source:

Protocol \#: $\quad 1103009531$

Expiration Date: $\quad$ April 05, 2012

Risk Level / Category: Research not involving greater than minimal risk

The above-referenced protocol amendment, as itemized below, was reviewed by the Chairperson/designee of the Wayne State University Institutional Review Board (B3) and is APPROVED effective immediately.

- Participant Information/brochure/pamphlet- Addition of cover letter to be provided to experts to complete the second round data collection.

- Protocol- Changes to data collection methods and/or instruments include an increase in the number of concept pairs from 45 to 55 .

- Information Sheet (dated 06/27/2011)- Research Information Sheet (Participants) updated to reflect increase in the number of questions on the second part of the questionnaire. 


\section{APPENDIX B: Information Sheets for Participation to Research}

\section{Research Information Sheet (Experts)}

The Mental Model Comparison of Expert and Novice Performance Improvement Practitioners

Principal Investigator (PI): $\quad$ Sacip Toker Instructional Technology

(313) 645-7112

\section{Purpose:}

You are being asked to participate in a research study revealing expertise in Human Performance Improvement (HPT) and factors affecting it because:

1. You have written extensively in the field of performance improvement,

2. You have been active in professional organizations, such as International Society for Performance Improvement (ISPI), The American Society for Training and Development (ASTD), or Association for Educational Communication and Technology's (AECT) Training and Performance Improvement track, among others.

3. You are recommended by persons identified via the first two criteria, and

4. You have ten or more years experience in the performance improvement field.

This study is being conducted at Wayne State University in conjunction with doctoral research.

\section{Study Procedures:}

If you take part in the study, you will be asked to complete two online questionnaires on two different occasions. The first online questionnaire includes a list of 30 concepts related to the HPT field. These 30 terms were identified after the review of seminal publications. You will rank 10 of 30 concepts according to their importance. The second questionnaire will include 45 concept pairs, which will be generated from the answers to the first questionnaire, and the professional profile characteristics. It will be sent out two weeks after the first questionnaire. All concepts pairs will be answered according to their relationships, and the professional profile characteristics questions will be answered based on instructions. If you are willing to participate in the study, all questions have to be answered. Both questionnaires will take 15-20 minutes to complete. The results of these two questionnaires will be utilized to create a reference expert model for comparison purposes.

\section{Benefits}


As a participant in this research study, there may be no direct benefit for you; however, information from this study may benefit other people now or in the future. The study will add to the body of research in the field of Human Performance Improvement, specifically in the area of human expertise and the factors affecting it.

\section{Risks}

There are no known risks at this time to participation in this study.

\section{Costs}

There will be no costs to you for participation in this research study.

\section{Compensation}

You will not be paid for taking part in this study. However, we will be happy to provide each participant with a copy of the summary results, if so requested.

\section{Confidentiality:}

You will be identified in the research records by a code name or number. There will be a password protected excel file containing the code indentified information. It will be kept on the Pl's password protected computers and secured storage device. Only the PI will have access to this document. Once the data collection is completed, the code identifier document will be deleted from the Pl's personal laptop and secure storage.

\section{Voluntary Participation /Withdrawal:}

Taking part in this study is voluntary. You may choose not to take part in this study, or if you decide to take part, you can change your mind later and withdraw from the study. You are free to not answer any questions or withdraw at any time. Your decision will not change any present or future relationships with Wayne State University or its affiliates.

\section{Questions:}

If you have any questions about this study now or in the future, you may contact Sacip Toker at the following phone number (313) 645-7112. If you have questions or concerns about your rights as a research participant, the Chair of the Human Investigation Committee can be contacted at (313) 577-1628. If you are unable to contact the research staff, or if you want to talk to someone other than the research staff, you may also call (313) $577-1628$ to ask questions or voice concerns complaints.

\section{Participation:}

By completing the two online questionnaires you are agreeing to participate in this study. 


\section{Research Information Sheet (Participants)}

The Mental Model Comparison of Expert and Novice Performance Improvement Practitioners

Principal Investigator $(\mathrm{Pl}): \quad$ Sacip Toker Instructional Technology

(313) 645-7112

\section{Purpose:}

You are being asked to participate in a research study revealing the expertise in Human Performance Technology (HPT) and the factors affecting this expertise. You have been selected because you are currently a practitioner in the field in your role, as a student, an academician, or a professional. This study is being conducted at Wayne State University in conjunction with my doctoral studies.

\section{Study Procedures:}

If you take part in the study, you will be asked to complete an online questionnaire including professional profile characteristics and concept-pair items. The 22 professional profile characteristics questions are related to your professional activities in the field; the 55 concept-pairs questions, which were identified by experts, will be rated according to their relationships. If you willing to participate in the study, all questions have to be answered. The questionnaire will take 15-20 minutes to complete.

\section{Benefits}

As a participant in this research study, there may be no direct benefit for you; however, information from this study may benefit other people interested in HPT now or in the future. The study will add to the body of research in the field of Human Performance Technology, specifically in the area of human expertise and the factors affecting it.

\section{Risks}

There are no known risks at this time to participation in this study.

\section{Costs}

There will be no costs to you for participation in this research study.

\section{Compensation}

For taking part in this research study, you will be included in random drawing. At the end of the drawing, the selected 10 participants will be rewarded \$25 gift card for their time and inconvenience.

\section{Confidentiality:}

You will be identified in the research records by a code name or number if you are willing to provide your e-mail address for the gift card random drawing. If you win one of ten $\$ 25$ Amazon.com gift cards, you will be contacted for your mailing address, which 
will be destroyed after the gift is sent. There will be a password protected excel file containing the code identified information. It will be kept on the Pl's password protected computers and secured storage device. Only the PI will have access to this document. Once the data collection and random drawing are completed, the code identifier document will be deleted from the Pl's personal laptop and secure storage.

\section{Voluntary Participation /Withdrawal:}

Taking part in this study is voluntary. You are free not to answer any questions or withdraw at any time. Your decision will not change any present or future relationships with Wayne State University or its affiliates.

\section{Questions:}

If you have any questions about this study now or in the future, you may contact Sacip Toker at the following phone number (313) 645-7112. If you have questions or concerns about your rights as a research participant, the Chair of the Human Investigation Committee can be contacted at (313) 577-1628. If you are unable to contact the research staff, or if you want to talk to someone other than the research staff, you may also call (313) $577-1628$ to ask questions or voice concerns or complaints.

\section{Participation:}

By completing the online questionnaire you are agreeing to participate in this study. 


\section{Invitation to Participate in Research Study}

Dear HPT Expert,

My name is Sacip Toker. I am a doctoral candidate of Instructional Technology program at Wayne State University. Dr. James L. Moseley is my dissertation advisor.

I would like to invite you to participate in two consecutive online questionnaires of revealing the expertise in Human Performance Technology (HPT) and the factors affecting this expertise. The first questionnaire will take you approximately 10 - 15 minutes to complete. You will be contacted again to fill the second questionnaire two weeks later your completion of the first survey. The second survey will take 10 - 15 minutes to complete.

If you would like to participate in this survey, click on this link:

https://www.surveymonkey.com/s/the_study_phase1_round1

In order to contact you for the second questionnaire, your e-mail information will be kept temporarily. Once the data collection with second questionnaire is completed, this information will be removed.

Please email or call me if you have questions on participating in or learning more about this dissertation study. I may be reached at saciptoker@gmail.com or (313) 645-7112.

Sincerely,

Sacip Toker

Doctoral Candidate

Wayne State University 


\section{Invitation to Participate in Research Study - The Second Questionnaire}

Dear HPT Expert,

My name is Sacip Toker. I am a doctoral candidate in the Instructional Technology program at Wayne State University. Dr. James L. Moseley is my dissertation advisor.

You have previously been contacted, and you completed the first questionnaire revealing the expertise in Human Performance Technology (HPT) and the factors affecting this expertise. You will recall the first questionnaire was about identification and ranking of 10 important concepts for the field.

I would like to invite you to participate in the second questionnaire that will take 15 - 20 minutes to complete. The second questionnaire includes the professional profile characteristics and concept-comparison sections.

If you would like to participate in this survey, click on this link:

https://www.surveymonkey.com/s/the_study_phase1_round2

Please email or call me if you have questions on participating in or learning more about this dissertation study. I may be reached at saciptoker@gmail.com or (313) 645-7112.

Sincerely,

Sacip Toker

Doctoral Candidate

Wayne State University

Detroit, MI 


\title{
Invitation to Participate in Research Study
}

\author{
Dear HPT Professionals,
}

My name is Sacip Toker. I am a doctoral candidate of Instructional Technology program at Wayne State University. Dr. James L. Moseley is my dissertation advisor.

I would like to invite you to participate in an online questionnaire of revealing the expertise in Human Performance Technology (HPT) and the factors affecting this expertise. It will take you approximately 20 - 30 minutes to complete this questionnaire. Participants will have the option of entering a random drawing to receive one of ten $\$ 25$ gift cards.

If you would like to participate in this survey, click on this link:

https://www.surveymonkey.com/s/the_study_phase2

If you choose to participate in the drawing, you will need to provide your email address on the questionnaire. This information will be kept separately from the survey information, and it will be deleted once the drawing is completed. You will be contacted for your mailing address if you are selected as one of the gift card winners. This information will be deleted after gift cards are mailed.

Please email or call me if you have questions on participating in or learning more about this dissertation study. I may be reached at saciptoker@gmail.com or (313) 645-7112.

Sincerely,

Sacip Toker

Doctoral Candidate

Wayne State University 


\section{Invitation to Participate in Research Study}

Dear HPT Professionals,

My name is Sacip Toker. I am a doctoral candidate of Instructional Technology program at Wayne State University. Dr. James L. Moseley is my dissertation advisor.

I would like to invite you to participate in an online questionnaire of revealing the expertise in Human Performance Technology (HPT) and the factors affecting this expertise. It will take you approximately 20 - 30 minutes to complete this questionnaire. Participants will have the option of entering a random drawing to receive one of ten \$25 gift cards.

If you would like to participate in this survey, click on this link:

https://www.surveymonkey.com/s/the_study_phase2

Please email or call me if you have questions on participating in or learning more about this dissertation study. I may be reached at saciptoker@gmail.com or (313) 645-7112.

Sincerely,

Sacip Toker

Doctoral Candidate

Wayne State University 
APPENDIX D: The Support Letters from the Professional Organizations

International Society for Performance Improvement (ISPI)

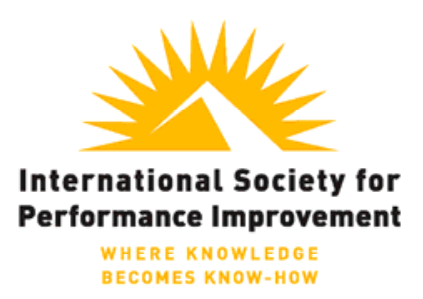

Sacip Toker

Graduate Research Assistant

Wayne State University

1261 Kirts Blvd, Apt 141

Troy, MI 48084

313.645 .7112

saciptoker@gmail.com

February 3, 2011

RE: Permission for participation in research study, The Mental Model Comparison of Expert and Novice Performance Improvement Practitioners, by Sacip Toker

Dear Sacip,

This letter is in response to your inquiry to use conduct the research study, The Mental Model Comparison of Expert and Novice Performance Improvement Practitioners, of the members and non-members of the International Society for Performance Improvement (ISPI).

We have reviewed your request and hereby grant you limited permission to conduct a research study through our distribution list.

ISPI will permit Sacip Toker to include a letter of invitation, with a description of the survey in our monthy electronic newsletter, PerformanceXpress, inviting readers to click on a link to an external survey website. The newsletter is distributed to approximately 23,000 email addresses. ISPI makes no claims, promises, or guarantees regarding the sample size and/or population that will participate in the survey or any other type of response rate.

The researcher will be permitted to include a statement of no more than 250 words and must include contact information, a link to an external survey, a description of the research project, and letter of invitation for participation. This letter may be included within our newsletter for a maximum of 3 issues/months or less upon request by the researcher.

The researcher may also request participation through our monthly Certified Performance Technologist (CPT) newsletter with a description of the survey and a link to external survey website. The research will be permitted a call of no more than 150 words in the CPT newsletter.

ISPI will not provide any individual contact information for any of its members and/or non-members. If the researcher wishes to obtain any contact or personal information, it must be requested in the survey and through volunteer/opt-in only process. ISPI is only permitting the use of the our newsletter, PerformanceXpress, to distribute an invitation for participation.

If you have any questions or concerns please feel free to contact me.

Sincerely,

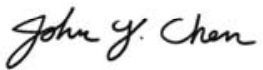

John Chen

Publications Manager

International Society for Performance Improvement 


\section{The American Society for Training and Development (ASTD)}

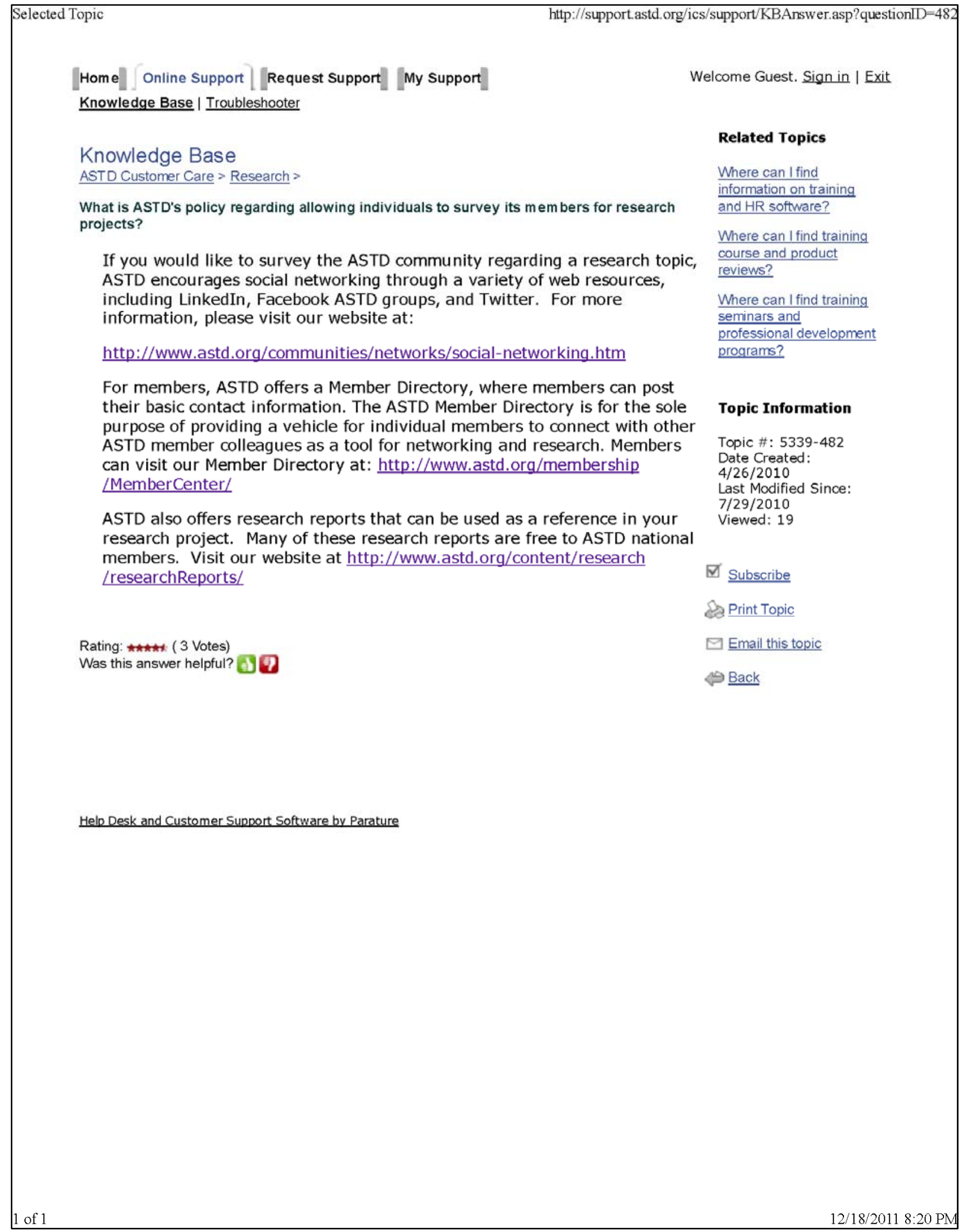




\section{Association for Education Communications and Technology (AECT)}

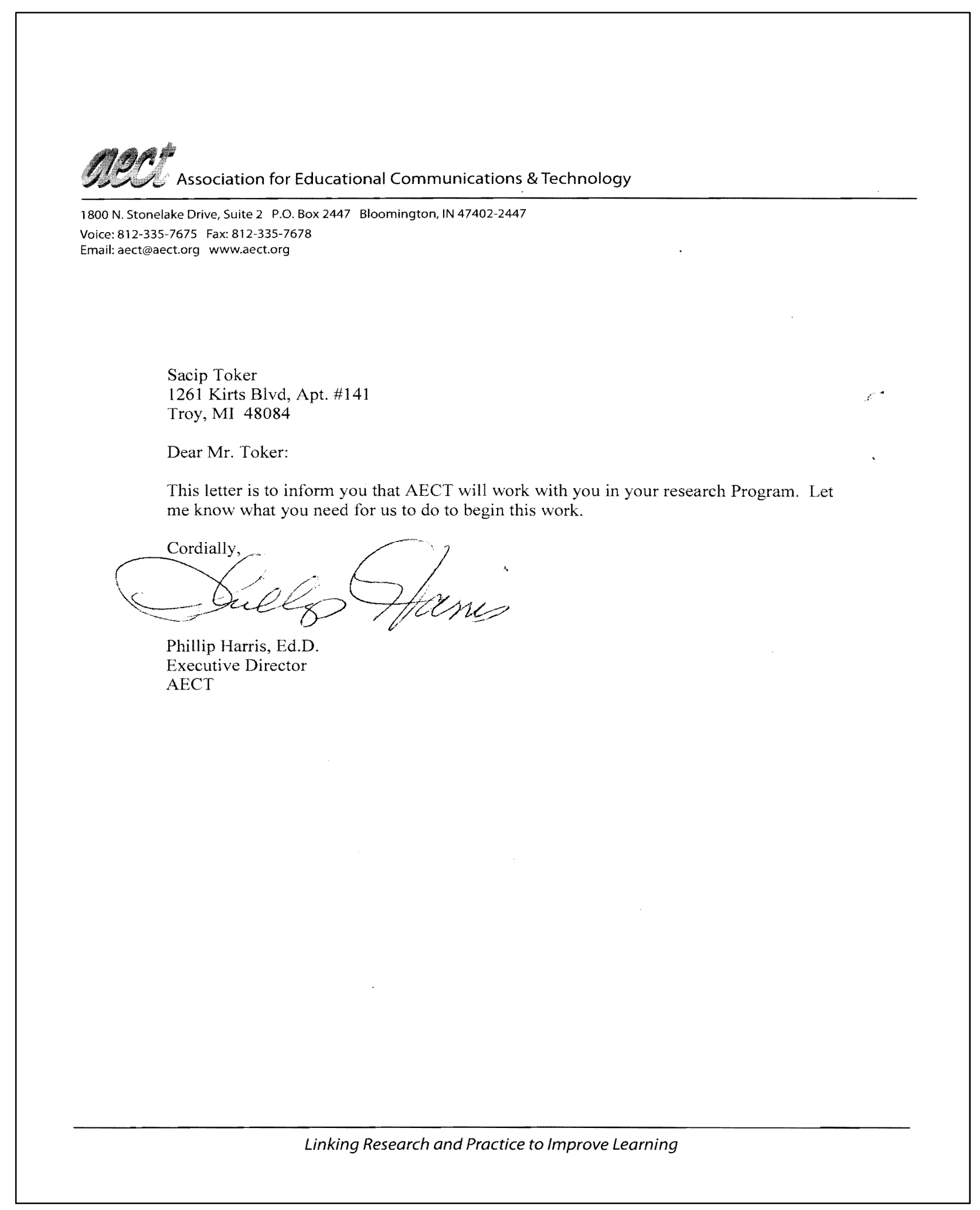




\section{Policy on Requesting AECT Member Participation in Research Studies}

From time to time, AECT receives formal requests for email or mailing lists for the purpose of soliciting AECT members to participate in surveys and other studies. In some cases, these requests ask AECT Headquarters to help draw random or purposeful samples. Additionally, the requested sample sometimes seems best derived from the total membership and other times might best be drawn from a division or cluster of divisions.

As an international organization, AECT is an active supporter of research and wishes to facilitate research studies and sharing of results that may benefit the field. At the same time, AECT is responsible for assuring that its members receive no more requests for participation than are reasonable and that such participation requests are appropriate. AECT also has the responsibility of assuring its membership rolls are properly protected.

In order to help identify whether a request for a sample is appropriate for member participation and to facilitate deriving the proper sample, those requesting samples should provide the following information as part of their requests:

1. The title of the study for which a sample is requested.

2. The name, affiliation, title, and contact information of the requesting person or organization.

3. Whether the requester is a member of AECT, and if so, any divisions with which affiliated.

4. The size and nature of the requested sample.

5. Why AECT member participation is appropriate.

6. Specifics on those for whom participation might be most appropriate (for instance, age, gender, race, rank, experience, content area, type of employment, etc.)

7. Why the study is important to the field and why its results would be of interest/benefit to AECT members.

8. A description of how the results are to be used.

9. The name, affiliation, title, and contact information for advisors, chairs, or other supervisors involved.

10. An assurance statement that confirms the researcher(s) involved will not share participant data or participant addresses or emails, that contact information for participants will be retained under lock and key, and that such contact information will be destroyed upon completion of the research.

Upon receipt of such a request, the Executive Committee of the AECT Board will discuss the merits of the research and decide whether AECT should facilitate member participation in the proposed research. If participation does seem appropriate, the Executive Committee -in consultation with division officers and headquarters staff- will decide whether sampling is most reasonable at the organizational level or the divisional level, or some other level (for example, Special Interest Forum, Task Force, or Work Group). If the Exec feels the request needs modification before AECT can facilitate participation or if AECT needs further information (such as confirmation by the requester's affiliated organization), the requester may be asked to make such modifications or supply such additional information prior to approval. 
Once AECT agrees to supply a sample list, the researcher needs to file the following materials with AECT Headquarters prior to release of the member-sampling list to the researcher:

11. A copy of approval by appropriate certifying panels or committees (such as Human Subjects Review or Institutional Research Boards), when such panels or committees have authority over the research.

12. Copies of all instruments to be used with AECT members. 
The Application of Request Permission to Conduct Research with AECT Training and Performance Division Members for the Present Study

1. The title of the study for which a sample is requested.

THE MENTAL MODEL COMPARISON OF EXPERT AND NOVICE PERFORMANCE IMPROVEMENT PRACTITIONERS

2. The name, affiliation, title, and contact information of the requesting person or organization.

Sacip Toker

Wayne State University

Graduate Research Assistant

E-mail: saciptoker@gmail.com

Phone: (313) 6457112

Fax: (313) 5771693

Address: 1261 Kirts Blvd, Apt 141, Troy, MI, 48084

\section{The size and nature of the requested sample.}

The researcher will send out an online survey to the current members of Training and Performance division of AECT. The participation will be voluntary. There will be two sample groups required for the study. The first sample group includes experts in training and performance improvement field who meet the following criteria:

1. Those who had written extensively in the field of performance improvement,

2. Those who have been active in the professional organizations,

3. Those who were recommended by persons identified via the first two criteria, and

4. Those who have had experience in performance improvement field for ten or more than ten years.

5. Those who had completed numerous HPT related projects.

The second sample group includes all members of Training and Performance division.

4. Why AECT member participation is appropriate.

AECT is one of the leading organizations in the field. Specifically, Training and Performance Division members are expected to be the professionals who work actively in the market, students who are pursuing their professional training, and scholars who teach or conduct research in the field. Since the proposed research focuses on expertise on training performance improvement practitioners, the Training and Performance Division members are very critical of the study to create an expert referent model regarding training and performance improvement and identify factors affecting expertise. 
5. Specifics on those for whom participation might be most appropriate (for instance, age, gender, race, rank, experience, content area, type of employment, etc.)

The most appropriate participant group will be the current members of Training and Performance Division, who would like to volunteer for participating in the study. The study needs both experts and novices from this field. The participants' experience in the field will be important for categorization.

6. Why the study is important to the field and why its results would be of interest/benefit to AECT members.

This study is very important to understand the expertise of professionals who currently work actively in the training and performance field. Moreover, the study will provide a clearer understanding of the performance improvement field. Comparison of experts and novices will enlighten the current degree programs in the field and improve the current educational and training techniques, methods, and curriculum. AECT members will be willing to help the field to develop the current theories and practices. The expected contributions of the proposed study will also help to improve the current context of educational institutions as well as the professionals who graduated from these institutions. There is a pending dissertation support grant for the study. If it is accepted, 60 participants will be rewarded with $\$ 25$ gift card; the awardees will be selected randomly.

\section{A description of how the results are to be used.}

The results will be utilized for the completion of a doctoral dissertation in the short term. In the long term, they will be published in scholarly journals.

8. The name, affiliation, title, and contact information for advisors, chairs, or other supervisors involved.

James L. Moseley, Ed.D. (Advisor and Chair of Dissertation Committee)

Wayne State University

Associate Professor

E-mail: moseley@wayne.edu

Phone: (313) 5777948

Fax: (313) 5771693

Ingrid Guerra-Lopez, Ph.D. (Member of Dissertation Committee)

E-mail: iguerra@wayne.edu

Wayne State University

Associate Professor

Phone: (313) 5771728

Fax: (313) 5771693

Timothy W. Spannaus, Ph.D. (Member of Dissertation Committee)

Wayne State University 
Sr. Lecturer \& Program Coordinator

E-mail: tspannaus@wayne.edu

Phone: (313) 5771741

Fax: (313) 5771693

Celia Livermore, Ph.D. (Member of Dissertation Committee)

Wayne State University

Professor of Information Systems Management

E-mail: ak1667@wayne.edu

Phone: (313) 5772243

The primary investigator of the study will not share participant data or participant addresses or emails. Contact information for participants will be retained under lock and key, and that such contact information will be destroyed upon completion of the research.

All information collected during the course of this study will be retained without any identifiers. 


\section{APPENDIX E: The Online Ranking Questionnaire}

Directions:

A. Please review the following 30 concepts

B. Identify 10 concepts out of 30 that are more critical than others for the understanding of the HPT field:

\begin{tabular}{|l|l|}
\hline Added-value & Intervention Implementation \\
\hline Appreciative Inquiry & Intervention Selection, Design \& Development \\
\hline Cause Analysis & Knowledge \& Skills \\
\hline Communities of Professional Practice & Needs Assessment \\
\hline Cost-Effectiveness & Performance Analysis \\
\hline Critical Business Issue(s) & Return on Investment \\
\hline Establishing Partnership with Client & Strategic Planning \\
\hline Ethical Dimensions & Strategic Thinking \\
\hline Measurement \& Evaluation & Sufficient Resources \\
\hline Financial Management & Systematic Approach \\
\hline Focus on Results & Systems View \\
\hline Gap Analysis & Talent Management \\
\hline Incentives \& Motives & Team Development \\
\hline Individual Capacity & Training \\
\hline Instructional Design & Work, Worker \& Workplace \\
\hline
\end{tabular}

C. Rank the 10 concepts that you have selected by using the ranking scale below. All concepts are provided in the drop-down menus in alphabetically ascending order.

PLEASE USE ONE CONCEPT FOR ONLY ONE RANK AND COMPLETE ALL 10

RANKS. 
The concept ranking scale

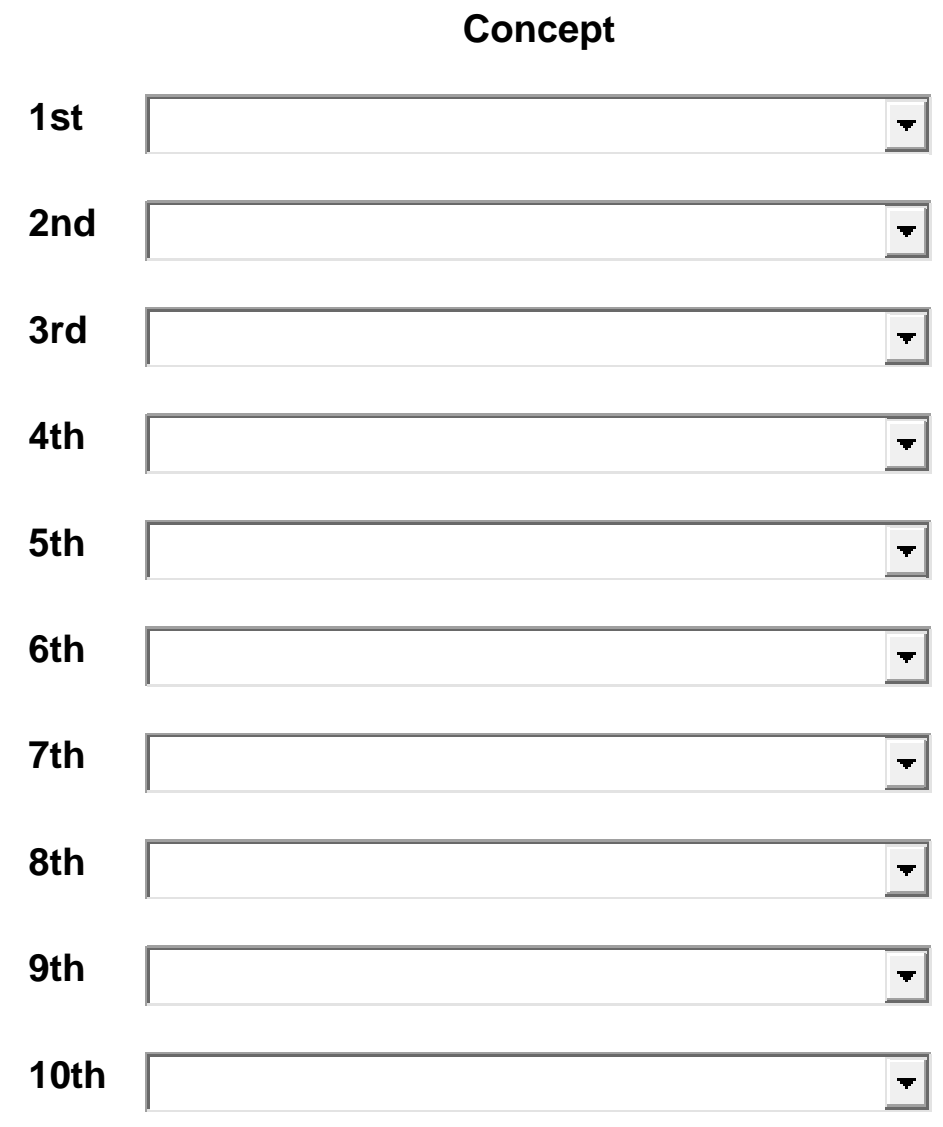

If you have additional comments, please indicate below: 


\section{APPENDIX F: The Concept-Pairs Comparison Questionnaire}

Direction: Direction: Please rate the relatedness of the concepts below. Concepts can be related in many ways - they can be in the same category, used in a similar way, or even related by time. For each of the pairs of terms listed below, select a number from 1 to 7 to indicate how related you think the concepts are. Smaller numbers mean very low relationship; median numbers mean moderate relationship; larger numbers mean very high relationship. Use what you have known about the concepts to make your ratings. Try not to spend more than 10 to 15 seconds to decide how related a pair is since your first impressions are usually more significant. Once you have selected a rating, choose the corresponding number. Please work quickly, but accurately.

\begin{tabular}{|c|c|c|c|c|c|c|c|}
\hline Comparisons & $\begin{array}{c}1 \\
\text { Very } \\
\text { low }\end{array}$ & 2 & 3 & \begin{tabular}{|c|}
4 \\
Moderate
\end{tabular} & 5 & 6 & $\begin{array}{c}7 \\
\text { Very } \\
\text { high }\end{array}$ \\
\hline \multicolumn{8}{|l|}{ Focus on Results - Performance Analysis } \\
\hline \multicolumn{8}{|l|}{ Focus on Results - Systems View } \\
\hline \multicolumn{8}{|l|}{ Focus on Results - Gap Analysis } \\
\hline \multirow{2}{*}{\multicolumn{8}{|c|}{ Focus on Results - Critical Business Issue(s) }} \\
\hline \multicolumn{4}{|l|}{ Focus on Results - Cause Analysis } & & & & \\
\hline \multirow{2}{*}{\multicolumn{8}{|c|}{$\begin{array}{l}\text { Focus on Results - Strategic Thinking } \\
\text { Focus on Results - Systematic Approach }\end{array}$}} \\
\hline Focus on Results - Systematic Approach & & & & & & & \\
\hline \multicolumn{8}{|l|}{$\begin{array}{l}\text { Focus on Results - Intervention Selection, Design } \\
\& \text { Development }\end{array}$} \\
\hline \multicolumn{8}{|l|}{$\begin{array}{l}\text { Focus on Results - Establishing Partnership with } \\
\text { Client }\end{array}$} \\
\hline \multicolumn{8}{|l|}{ Focus on Results - Measurement \& Evaluation } \\
\hline \multicolumn{8}{|l|}{ Performance Analysis - Systems View } \\
\hline \multirow{2}{*}{\multicolumn{8}{|c|}{ Performance Analysis - Gap Analysis }} \\
\hline & \multirow{2}{*}{\multicolumn{7}{|c|}{ Performance Analysis - Critical Business Issue(s) }} \\
\hline Performance Analysis - Cause Analysis & & & & & & & \\
\hline \multirow{2}{*}{\multicolumn{8}{|c|}{$\begin{array}{l}\text { Performance Analysis - Strategic Thinking } \\
\text { Performance Analysis - Systematic Approach }\end{array}$}} \\
\hline Performance Analysis - Systematic Approach & & & & & & & \\
\hline \multicolumn{8}{|l|}{$\begin{array}{l}\text { Performance Analysis - Intervention Selection, } \\
\text { Design \& Development }\end{array}$} \\
\hline \multicolumn{8}{|l|}{$\begin{array}{l}\text { Performance Analysis - Establishing Partnership } \\
\text { with Client }\end{array}$} \\
\hline \multicolumn{8}{|l|}{$\begin{array}{l}\text { Performance Analysis - Measurement \& } \\
\text { Evaluation }\end{array}$} \\
\hline \multicolumn{8}{|l|}{ Systems View - Gap Analysis } \\
\hline \multicolumn{8}{|l|}{ Systems View - Critical Business Issue(s) } \\
\hline \multirow{2}{*}{\multicolumn{8}{|c|}{ Systems View - Cause Analysis }} \\
\hline \multirow{2}{*}{\multicolumn{8}{|c|}{ Systems View - Strategic Thinking }} \\
\hline Systems View - Systematic Approach & & & & & & & \\
\hline \multicolumn{8}{|l|}{$\begin{array}{l}\text { Systems View - Intervention Selection, Design \& } \\
\text { Development }\end{array}$} \\
\hline \multicolumn{8}{|l|}{$\begin{array}{l}\text { Systems View - Establishing Partnership with } \\
\text { Client }\end{array}$} \\
\hline Systems View - Measurement \& Evaluation & & & & & & & \\
\hline
\end{tabular}




\begin{tabular}{|c|c|c|c|c|c|c|c|}
\hline Comparisons & $\begin{array}{c}1 \\
\text { Very } \\
\text { low }\end{array}$ & 2 & 3 & \begin{tabular}{|c|}
4 \\
Moderate
\end{tabular} & 5 & 6 & $\begin{array}{c}7 \\
\text { Very } \\
\text { high }\end{array}$ \\
\hline \multicolumn{8}{|l|}{ Gap Analysis - Critical Business Issue(s) } \\
\hline \multicolumn{8}{|l|}{ Gap Analysis - Cause Analysis } \\
\hline \multicolumn{8}{|l|}{ Gap Analysis - Strategic Thinking } \\
\hline \multicolumn{8}{|l|}{ Gap Analysis - Systematic Approach } \\
\hline \multicolumn{8}{|l|}{$\begin{array}{l}\text { Gap Analysis - Intervention Selection, Design \& } \\
\text { Development }\end{array}$} \\
\hline \multicolumn{8}{|l|}{$\begin{array}{l}\text { Gap Analysis - Establishing Partnership with } \\
\text { Client }\end{array}$} \\
\hline \multicolumn{8}{|l|}{ Gap Analysis - Measurement \& Evaluation } \\
\hline \multicolumn{8}{|l|}{ Critical Business Issue(s) - Cause Analysis } \\
\hline \multirow{2}{*}{\multicolumn{8}{|c|}{ Critical Business Issue(s) - Strategic Thinking }} \\
\hline & & & & & & & Critical Business Issue(s) - Systematic Approach \\
\hline \multicolumn{8}{|l|}{$\begin{array}{l}\text { Critical Business Issue(s) - Intervention Selection, } \\
\text { Desian \& Develooment }\end{array}$} \\
\hline \multicolumn{8}{|l|}{$\begin{array}{l}\text { Critical Business Issue(s) - Establishing } \\
\text { Partnership with Client }\end{array}$} \\
\hline \multicolumn{8}{|l|}{$\begin{array}{l}\text { Critical Business Issue(s) - Measurement \& } \\
\text { Evaluation }\end{array}$} \\
\hline \multicolumn{8}{|l|}{ Cause Analysis - Strategic Thinking } \\
\hline \multicolumn{8}{|l|}{ Cause Analysis - Systematic Approach } \\
\hline \multicolumn{8}{|l|}{$\begin{array}{l}\text { Cause Analysis - Intervention Selection, Design \& } \\
\text { Development }\end{array}$} \\
\hline \multicolumn{8}{|l|}{$\begin{array}{l}\text { Cause Analysis - Establishing Partnership with } \\
\text { Client }\end{array}$} \\
\hline \multicolumn{8}{|l|}{ Cause Analysis - Measurement \& Evaluation } \\
\hline \multicolumn{8}{|l|}{ Strategic Thinking - Systematic Approach } \\
\hline \multicolumn{8}{|l|}{$\begin{array}{l}\text { Strategic Thinking - Intervention Selection, } \\
\text { Design \& Development }\end{array}$} \\
\hline \multicolumn{8}{|l|}{$\begin{array}{l}\text { Strategic Thinking - Establishing Partnership with } \\
\text { Client }\end{array}$} \\
\hline \multicolumn{8}{|l|}{ Strategic Thinking - Measurement \& Evaluation } \\
\hline \multicolumn{8}{|l|}{$\begin{array}{l}\text { Systematic Approach - Intervention Selection, } \\
\text { Design \& Development }\end{array}$} \\
\hline \multicolumn{8}{|l|}{$\begin{array}{l}\text { Systematic Approach - Establishing Partnership } \\
\text { with Client }\end{array}$} \\
\hline \multicolumn{8}{|l|}{$\begin{array}{l}\text { Systematic Approach - Measurement \& } \\
\text { Evaluation }\end{array}$} \\
\hline \multicolumn{8}{|l|}{$\begin{array}{l}\text { Intervention Selection, Design \& Development - } \\
\text { Establishing Partnership with Client }\end{array}$} \\
\hline \multicolumn{8}{|l|}{$\begin{array}{l}\text { Intervention Selection, Design \& Development - } \\
\text { Measurement \& Evaluation }\end{array}$} \\
\hline $\begin{array}{l}\text { Establishing Partnership with Client - } \\
\text { Measurement \& Evaluation }\end{array}$ & & & & & & & \\
\hline
\end{tabular}




\section{APPENDIX G: The Professional Profile Characteristics Questionnaire}

Please answer each question with the options provided. Please answer all questions. When you have completed all items, please click "Submit" button at the bottom of the page

1. How long have you been actively in the HPT field?
01 to 5 years
06 to 10 years
011 to 15 years
016 to 20 years
0 More than 20 years

Please read the following explanation:

Deliberate practice, which requires considerable, precise, and continuous efforts to do something an individual is not good at partially or at all, focuses on improving the current skills and advancing the accomplishments and boundaries of the skills. It also provides repeated experiences so individuals can grasp critical aspects of situations and incrementally increase their performances.

Routine work activities are not considered as deliberate practice since they are services rendered for pay and activities directly motivated to external rewards. Even though work activities offer some opportunities for learning and improving skills, they are not sufficient.

2. Please indicate percent of your professional activities corresponding with the explanation provided above.
$00 \%$
O $10 \%$
O $20 \%$
O $30 \%$
O $40 \%$
O $50 \%$
O $60 \%$
O $70 \%$
O $80 \%$
O $90 \%$
O $100 \%$

3. What is your current industry? 

O Agriculture
O Construction
O Higher Education (College/University)
O K-12 Education
O Insurance
O Government
0 Non-profit
O Retail or Wholesale
O Transportation
O Internet or Information Technology
0 Finance
O Real Estate
O Healthcare
O Manufacturing
O Services
O Communication and Utilities
O Military
O Independent Consultant (e.g. Performance consulting)
0 Other (Please specify)

4. Please choose the industries in which you have worked or completed a project. Please check all that apply:

- Agriculture

- Construction

- Higher Education (College/University)

- K-12 Education

- Insurance

- Government

- Non-profit

- Retail or Wholesale 
- Transportation

- Internet or Information Technology

- Finance

- Real Estate

- Healthcare

- Manufacturing

- Services

- Communication and Utilities

- Military

- Independent Consultant (e.g. Performance consulting)

- Other (Please specify)

5. How many different organizations have you worked so far?

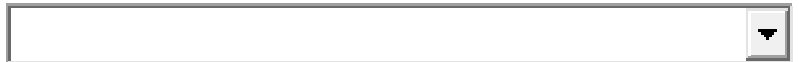

6. How many HPT related projects have you completed so far? Please indicate estimate the total numbers. If you have not completed any projects, please type "0":

7. What kinds of HPT related project(s) have you worked? Please check all that apply.

- I have not worked on any HPT related projects

- Needs Assessment

- Performance Analysis

- Instructional Design

- Instructional Development

- Intervention Design \& Development

- Intervention Implementation

- Measurement and Evaluation 
- Other (please specify)

8. Which of the following degree(s) do you have or are you pursuing? Please indicate the number of years it took or you have spent so far to each degree

\begin{tabular}{|l|c|c|c|c|c|c|c|}
\hline \multirow{2}{*}{ Degrees } & \multicolumn{9}{|c|}{ Years } \\
\cline { 2 - 7 } & 1 years & 2 years & 3 years & 4 years & 5 years & $\begin{array}{c}\text { More } \\
\text { than 5 } \\
\text { years }\end{array}$ & $\begin{array}{c}\text { Not } \\
\text { Applicable }\end{array}$ \\
\hline $\begin{array}{l}\text { Associate's } \\
\text { degree }\end{array}$ & $\square$ & $\square$ & $\square$ & $\square$ & $\square$ & $\square$ & $\square$ \\
\hline $\begin{array}{l}\text { Some } \\
\text { college, no } \\
\text { degree }\end{array}$ & $\square$ & $\square$ & $\square$ & $\square$ & $\square$ & $\square$ & $\square$ \\
\hline $\begin{array}{l}\text { Bachelor's } \\
\text { degree }\end{array}$ & $\square$ & $\square$ & $\square$ & $\square$ & $\square$ & $\square$ & $\square$ \\
\hline $\begin{array}{l}\text { Master's } \\
\text { degree }\end{array}$ & $\square$ & $\square$ & $\square$ & $\square$ & $\square$ & $\square$ & $\square$ \\
\hline $\begin{array}{l}\text { Educational } \\
\text { Specialist }\end{array}$ & $\square$ & $\square$ & $\square$ & $\square$ & $\square$ & $\square$ & $\square$ \\
\hline Doctorate & $\square$ & $\square$ & $\square$ & $\square$ & $\square$ & $\square$ & $\square$ \\
\hline $\begin{array}{l}\text { Postgraduate } \\
\text { study }\end{array}$ & $\square$ & $\square$ & $\square$ & $\square$ & $\square$ & $\square$ & $\square$ \\
\hline
\end{tabular}

9. If you received any training specific to HPT and/or HPT related activities in addition to your degrees, please indicate estimate the total hours you spent. If you have not received any training type "0": 
10. Which of the following certificates do you have? Check all that apply.

- I don't have any certification

- Certified Performance Technologist (CPT)

- Certified Professional in Learning and Performance Certification (CPLP)

- Other (please specify):

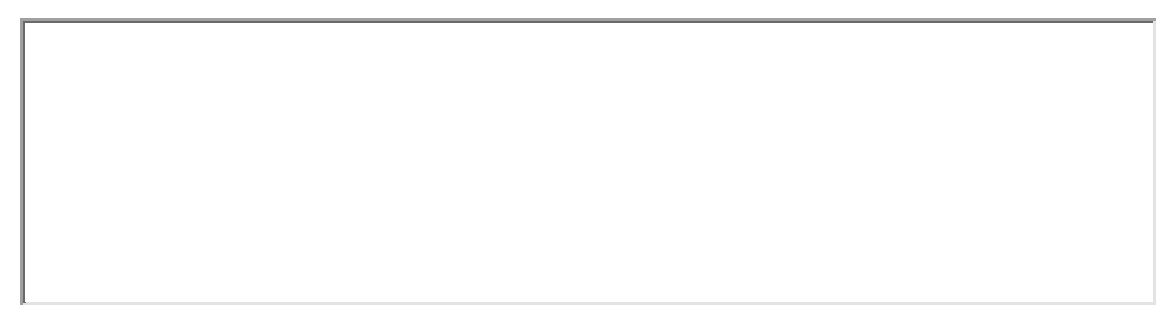

11. Please indicate your number of publications by type. Indicating " 0 " means no publications.

Books

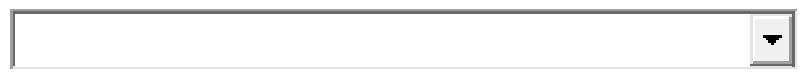

Book Chapters

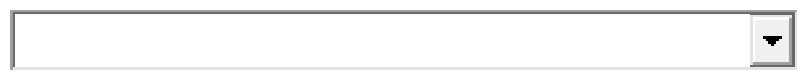

Refereed journals

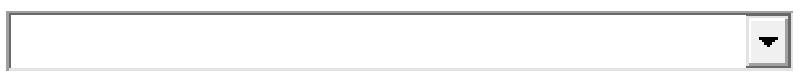

Non-refereed journals

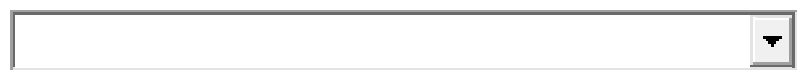

Proceedings

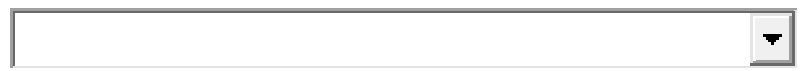

Poster sessions

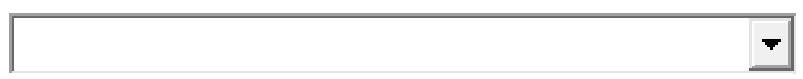

Educational/Instructional Materials

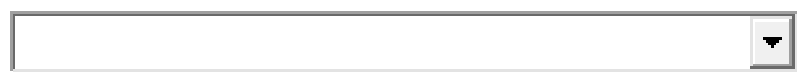

Book Reviews

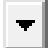


12. How many professional presentations (i.e., how-to, educational/lecture, case study, business, futurecasting, issues, etc.) or workshops have you done so far? "0" indicates no participation.

Presentations

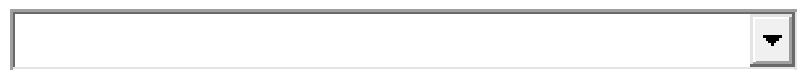

Workshops

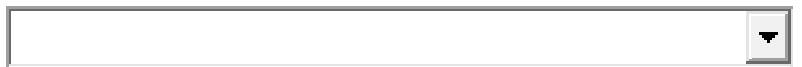

13. If you teach any HPT related courses, please indicate the number of courses by course format? " 0 " indicates no courses taught.

Face to Face

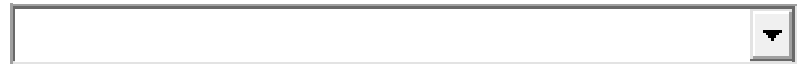

On-line

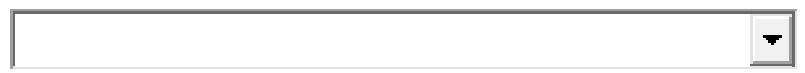

Blended

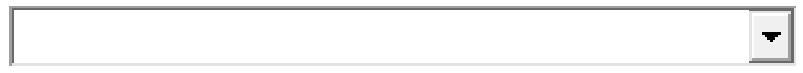

14. Are you a member of the following organizations? Please check all that apply:
ISPI - International
ISPI - Local chapter
ASTD - International
ASTD - Local chapter
AECT - International
AECT - Local chapter
No membership
Other (please specify) 
15. Please indicate an approximate number of times you participated in the following conferences?

\begin{tabular}{|l|c|c|c|c|c|c|c|}
\hline \multirow{2}{*}{ Conferences } & \multicolumn{7}{|c|}{ Number of times } \\
\cline { 2 - 8 } & $\begin{array}{c}\text { Not } \\
\text { participated }\end{array}$ & $\mathbf{1 - 5}$ & $\begin{array}{c}\mathbf{6}- \\
\mathbf{1 0}\end{array}$ & $\begin{array}{c}\mathbf{1 1}- \\
\mathbf{1 5}\end{array}$ & $\begin{array}{c}\mathbf{1 6}-\mathbf{2 1} \\
\mathbf{2 0}\end{array}$ & $\begin{array}{c}\text { More } \\
\mathbf{2 5} \\
\text { than 25 }\end{array}$ \\
\hline $\begin{array}{l}\text { ISPI - The Annual } \\
\text { Performance Improvement } \\
\text { Conference }\end{array}$ & $\square$ & $\square$ & $\square$ & $\square$ & $\square$ & $\square$ & $\square$ \\
\hline $\begin{array}{l}\text { ISPI - The local chapter } \\
\text { conferences, seminars, \& } \\
\text { workshops }\end{array}$ & $\square$ & $\square$ & $\square$ & $\square$ & $\square$ & $\square$ & $\square$ \\
\hline $\begin{array}{l}\text { ASTD - Annual International } \\
\text { Conference \& Exposition }\end{array}$ & $\square$ & $\square$ & $\square$ & $\square$ & $\square$ & $\square$ & $\square$ \\
\hline $\begin{array}{l}\text { ASTD - The local chapter } \\
\text { conferences, seminars, \& } \\
\text { workshops }\end{array}$ & $\square$ & $\square$ & $\square$ & $\square$ & $\square$ & $\square$ & $\square$ \\
\hline $\begin{array}{l}\text { AECT - Annual International } \\
\text { Convention }\end{array}$ & $\square$ & $\square$ & $\square$ & $\square$ & $\square$ & $\square$ & $\square$ \\
\hline $\begin{array}{l}\text { AECT - The local chapter } \\
\text { conferences, seminars, \& } \\
\text { workshops }\end{array}$ & $\square$ & $\square$ & $\square$ & $\square$ & $\square$ & $\square$ & $\square$ \\
\hline
\end{tabular}

16. If you are or have ever been a board member of the following professional organizations, please indicate your years of service:

\begin{tabular}{|l|c|c|c|c|c|c|c|}
\hline \multirow{2}{*}{ Organizations } & \multicolumn{7}{|c|}{ Years } \\
\cline { 2 - 7 } & $\mathbf{1}$ & $\mathbf{2}$ & $\mathbf{3}$ & $\mathbf{4}$ & $\mathbf{5}$ & $\begin{array}{c}\text { More than } \\
\mathbf{5}\end{array}$ & $\begin{array}{c}\text { Not a } \\
\text { board } \\
\text { member }\end{array}$ \\
\hline $\begin{array}{l}\text { ISPI - } \\
\text { International }\end{array}$ & $\square$ & $\square$ & $\square$ & $\square$ & $\square$ & $\square$ & $\square$ \\
\hline $\begin{array}{l}\text { ISPI - Local } \\
\text { chapter }\end{array}$ & $\square$ & $\square$ & $\square$ & $\square$ & $\square$ & $\square$ & $\square$ \\
\hline $\begin{array}{l}\text { ASTD - } \\
\text { International }\end{array}$ & $\square$ & $\square$ & $\square$ & $\square$ & $\square$ & $\square$ & $\square$ \\
\hline $\begin{array}{l}\text { ASTD - Local } \\
\text { chapter }\end{array}$ & $\square$ & $\square$ & $\square$ & $\square$ & $\square$ & $\square$ & $\square$ \\
\hline $\begin{array}{l}\text { AECT - } \\
\text { International }\end{array}$ & $\square$ & $\square$ & $\square$ & $\square$ & $\square$ & $\square$ & $\square$ \\
\hline $\begin{array}{l}\text { AECT - Local } \\
\text { chapter }\end{array}$ & $\square$ & $\square$ & $\square$ & $\square$ & $\square$ & $\square$ & $\square$ \\
\hline
\end{tabular}


17. If you have ever volunteered for the following professional organizations, please indicate how many times you volunteered.

\begin{tabular}{|l|c|c|c|c|c|c|c|}
\hline \multirow{2}{*}{ Organizations } & \multicolumn{7}{|c|}{ Number of times } \\
\cline { 2 - 8 } & 1 & 2 & 3 & 4 & 5 & $\begin{array}{c}\text { More } \\
\text { than 5 }\end{array}$ & $\begin{array}{c}\text { Not } \\
\text { volunteered }\end{array}$ \\
\hline ISPI - International & $\square$ & $\square$ & $\square$ & $\square$ & $\square$ & $\square$ & $\square$ \\
\hline $\begin{array}{l}\text { ISPI - Local } \\
\text { chapter }\end{array}$ & $\square$ & $\square$ & $\square$ & $\square$ & $\square$ & $\square$ & $\square$ \\
\hline $\begin{array}{l}\text { ASTD - } \\
\text { International }\end{array}$ & $\square$ & $\square$ & $\square$ & $\square$ & $\square$ & $\square$ & $\square$ \\
\hline $\begin{array}{l}\text { ASTD - Local } \\
\text { chapter }\end{array}$ & $\square$ & $\square$ & $\square$ & $\square$ & $\square$ & $\square$ & $\square$ \\
\hline $\begin{array}{l}\text { AECT - } \\
\text { International }\end{array}$ & $\square$ & $\square$ & $\square$ & $\square$ & $\square$ & $\square$ & $\square$ \\
\hline $\begin{array}{l}\text { AECT - Local } \\
\text { chapter }\end{array}$ & $\square$ & $\square$ & $\square$ & $\square$ & $\square$ & $\square$ & $\square$ \\
\hline
\end{tabular}

18. If you have ever received awards for your HPT professional activities, please indicate the "TYPES OF AWARDS" and "TOTAL NUMBERS" or type "NONE" if you have not been the recipient of rewards.

19. What is your current job title? 
20.What is your age?
0 Less than 30
O $31-40$
O $41-50$
O $51-60$
O $61-70$
O $71-80$
0 More than 80

21. What is your gender:
O Male
O Female

22. Please provide your e-mail address if you would like to be considered for $\$ 25$ retail store gift card sweepstake. This information will be used to re-contact with you if you win the award: 


\section{REFERENCES}

Acton, W. H., Johnson, P. J., \& Goldsmith, T. E. (1994). Structural knowledge assessment: comparison of referent structures. Journal of Educational Psychology, 86(2), 303-311.

Adelson, B. (1981). Problem solving and the development of abstract categories in programming languages. Mem Cognit, 9(4), 422-433.

Addison, R. M. \& Haig, C. (2006). The performance architect's essential guide to the performance technology landscape. In J. A. Pershing (Ed.) Handbook of human performance technology: principles, practices, and potential (3rd ed.), (pp. 3554). San Francisco, CA: Pfeiffer.

Alruwaii, A., \& Brooks, D. J. (2008). Organisational security: A propositional study to map expert knowledge. Paper presented at the The 1st Australian Security and Intelligence Conference, Perth Western Australia.

Atkinson, V., \& Chalmers, N. (1999). Performance consulting: Get your credit from your clients. Performance Improvement, 38(4), 14-19.

Barab, S., Warren, S. J., del Valle, R. \& Fang, F. (2006). Coming to terms with communities of practice: A definition and operational criteria. . In J. A. Pershing, (Ed.) Handbook of human performance technology: principles, practices, potential (3rd ed.), (pp 640-664). San Francisco, CA: Pfeiffer.

Beier, M. E., Campbell, M., \& Crook, A. E. (2010). Developing and demonstrating knowledge: Ability and non-ability determinants of learning and performance. Intelligence, 38(1), 179-186. doi: 10.1016/j.intell.2009.09.007 
Benner, P. (1984). From novice to expert: excellence and power in clinical nursing practice. Mento Park, CA: Addision-Wesley.

Bilalic, M., McLeod, P., \& Gobet, F. (2008). Inflexibility of experts--Reality or myth? Quantifying the Einstellung effect in chess masters. Cognitive Psychology, 56(2), 73-102. doi: 10.1016/j.cogpsych.2007.02.001

Boshuizen, H. P. A., \& Schmidt, H. G. (1992). On the role of biomedical knowledge in clinical reasoning by experts, intermediates and novices. Cognitive Science, 16(2), 153-184. doi: 10.1016/0364-0213(92)90022-m

Bradley, J. H., Paul, R., \& Seeman, E. (2006). Analyzing the structure of expert knowledge. Information \& Management, 43(1), 77-91. doi: 10.1016/j.im.2004.11.009

Brethower, D. M. (2000). Integrating theory, research, and practice in human performance technology: Examples from behavioral, cognitive, and constructivist theories. Performance Improvement, 39(4), 33-43.

Brockmole, J., Hambrick, D., Windisch, D., \& Henderson, J. (2008). The role of meaning in contextual cueing: Evidence from chess expertise. The Quarterly Journal of Experimental Psychology, 61(12), 1886-1896. doi: 10.1080/17470210701781155

Burkhardt, J.-M., Détienne, F., \& Wiedenbeck, S. (1997). Mental representations constructed by experts and novices in object-oriented program comprehension. Paper presented at the INTERACT'97, Sydney, Australia.

Burkhardt, J.-M., Détienne, F., \& Wiedenbeck, S. (2002). Object-Oriented Program Comprehension: Effect of Expertise, Task and Phase. Empirical Software Engineering, 7(2), 115-156. doi: 10.1023/a:1015297914742 
Burton, J. K., \& Merrill, P. F. (1991). Needs assessment: Goals, needs, and priorities. In L. J. Briggs, K. L. Gustafson, \& M. Tillman (Eds.), Instructional design: Principles and applications, (pp. 18-43). Englewood Cliffs, NJ: Educational Technology.

Butterworth, B. (2006). Mathematical Expertise. In R. R. Hoffmann (Ed.), The

Cambridge handbook of expertise and expert performance, (pp. 553-568). New York: Cambridge University.

Carley, K., \& Palmquist, M. (1992). Extracting, Representing, and Analyzing Mental Models. Social Forces, 70(3), 601-636.

Carroll, J. B. (1993). Human cognitive abilities: A survey of factor-analytic studies. New York: Cambridge University Press.

Castel, A. D., McCabe, D. P., Roediger, H. L., \& Heitman, J. L. (2007). The Dark Side of Expertise. Psychological Science, 18(1), 3-5. doi: 10.1111/j.1467$9280.2007 .01838 . x$

Charness, N., Krampe, R., \& Mayr, U. (1996). The role of practice and coaching in entrepreneurial skill domains: An international comparison of life-span chess skill acquisition. In K. A. Ericsson (Ed.), The road to excellence: The acquisition of expert performance in the arts and sciences, sports, and games (pp. 51-80). Mahwah, NJ: Erlbaum.

Charness, N., \& Tuffiash, M. (2008). The Role of Expertise Research and Human Factors in Capturing, Explaining, and Producing Superior Performance. Human Factors: The Journal of the Human Factors and Ergonomics Society, 50(3), 427432. doi: $10.1518 / 001872008 \times 312206$ 
Chevalier, R. (2008). The evolution of a performance analysis job aid. Performance Improvement, 47(10), 9-18.

Chi, M. T. H. (2006). Two approaches to the study of experts' characteristics. In R. R. Hoffmann (Ed.), The Cambridge handbook of expertise and expert performance (pp. 21-30). New York: Cambridge University.

Chi, M. T. H., Feltovich, P. J., \& Glaser, R. (1981). Categorization and representation of physics problems by experts and novices. Cognitive Science, 5(2), 121-152.

Chi, M. T. H., Glaser, R., \& Rees, E. (1982). Expertise in problem solving. In R. J. Stenberg (Ed.), Advances in the psychology of human intelligence (Vol. 1), (pp 775) Hillsdale, NJ: Erlbaum.

Chi, M. T. H., \& Koeske, R. D. (1983). Network representation of a child's dinosaur knowledge. Developmental Psychology, 19(1), 29-39. doi: Doi: 10.1037/00121649.19.1.29

Chiesi, H. L., Spilich, G. J., \& Voss, J. F. (1979). Acquisition of domain-related information in relationship to high and low domain knowledge. Journal of Verbal Learning and Verbal Behavior, 18(3), 257-273. doi: 10.1016/s0022$5371(79) 90146-4$

Chow, A.(2010). A needs assessment of the knowledge, skills and use of finance competencies by human performance technology practitioners. (Ph.D. dissertation, Wayne State University, United States - Michigan). Retrieved December 15, 2011, from Dissertations \& Theses @ Wayne State University.(Publication No. AAT 3412107). 
Cianciolo, A. T., Matthew, C., Sternberg, R. J., \& Wagner, R. K. (2006). Tacit Knowledge, Practical Intelligence, and Expertise. In R. R. Hoffmann (Ed.), The Cambridge handbook of expertise and expert performance (pp. 613-631). New York: Cambridge University.

Clariana, R., \& Wallace, P. (2007). A computer-based approach for deriving and measuring individual and team knowledge structure from essay questions. Journal of Educational Computing Research, 37(3), 211-227. doi: 10.2190/EC.37.3.a

Clariana, R., Wallace, P., \& Godshalk, V. (2009). Deriving and measuring group knowledge structure from essays: The effects of anaphoric reference. Educational Technology Research and Development, 57(6), 725-737. doi: $10.1007 / \mathrm{s} 11423-009-9115-z$

Clark, R. C. (2008). Building expertise cognitive methods for training and performance improvement (3rd ed.). San Francisco, CA: Pfeiffer.

Cleary, T. J., \& Zimmerman, B. J. (2001). Self-regulation differences during athletic practice by experts, non-experts, and novices. Journal of Applied Sport Psychology, 13, 185-206.

Cleary, T. J., Zimmerman, B. J., \& Keating, T. (2006). Training physical education students to self-regulate during basketball free throw practice. Research Quarterly for Exercise and Sport, 77(2), 251-262.

Cook, C., Heath, F., \& Thompson, R. L. (2000). A meta-analysis of response rates in web- or internet-based surveys. Educational and Psychological Measurement, 60(6), 821-836. doi: 10.1177/00131640021970934 
Cooke, N. J. (1994). Varieties of knowledge elicitation techniques. International Journal of Human-Computer Studies, 41(6), 801-849. doi: 10.1006/ijhc.1994.1083

Creswell, J. W. (2012). Educational research: planning, conducting, and evaluating quantitative and qualitative research (4th ed.). Boston, MA: Pearson Education Inc.

Day, D. V. and R. G. Lord (1992). Expertise and problem categorization: the role of expert processing in organizational sense-making. Journal of Management Studies, 29, 35-47.

Day, E. A., Arthur, W., Jr., \& Gettman, D. (2001). Knowledge structures and the acquisition of a complex skill. Journal of Applied Psychology, 86(5), 1022-1033. doi: 10.1037//0021-9010.86.5.1022

Dearholt, D. W., \& Schvaneveldt, R. W. (1990). Properties of pathfinder networks Pathfinder associative networks: studies in knowledge organization (pp. 1-30): Norwood, NJ: Ablex Publishing Corp.

Dew, N., Read, S., Sarasvathy, S. D., \& Wiltbank, R. (2009). Effectual versus predictive logics in entrepreneurial decision-making: Differences between experts and novices. Journal of Business Venturing, 24(4), 287-309. doi:

10.1016/j.jbusvent.2008.02.002

DiCerbo, K. E. (2007). Knowledge Structures of Entering Computer Networking Students and Their Instructors. Journal of Information Technology Education, 6, 263-277. 
Dorsey, D. W., Campbell, G. E., Foster, L. L., \& Miles, D. E. (1999). Assessing knowledge structures: relations with experience and posttraining performance. Human Performance, 12(1), 31 - 57.

Dreyfus, H. L., \& Dreyfus, S. D. (1986). Mind over machine. New York, NY: The Free Press.

Durso, F. T., \& Dattel, A. R. (2006). Expertise and Transportation. In R. R. Hoffmann (Ed.), The Cambridge handbook of expertise and expert performance (pp. 355372). New York: Cambridge University.

Endsley, M. R. (1988). Design and evaluation for situation awareness enhancement. Human Factors and Ergonomics Society Annual Meeting Proceedings, 32, 97101.

Endsley, M. R. (2006). Expertise and situation awareness. In R. R. Hoffmann (Ed.), The Cambridge handbook of expertise and expert performance (pp. 633-652). New York: Cambridge University.

Ericsson, K. A. (1998). The scientific study of expert levels of performance: general implications for optimal learning and creativity. High Ability Studies, 9(1), 75-100. doi: $10.1080 / 1359813980090106$

Ericsson, K. A. (2006). An introduction to Cambridge handbook of expertise and expert performance: its development, organization, and content. In R. R. Hoffmann (Ed.), The Cambridge handbook of expertise and expert performance (pp. 3-19). New York: Cambridge University.

Ericsson, K. A. (2008). Deliberate practice and acquisition of expert performance: a general overview. Academic Emergency Medicine, 15(11), 988-994. 
Ericsson, K. A., \& Charness, N. (1994). Expert performance: its structure and acquisition. American Psychologist, 49(80), 425-447.

Ericsson, K. A., Krampe, R. T., \& Tesch-Römer, C. (1993). The role of deliberate practice in the acquisition of expert performance. Psychological Review, 100(3), 363-406.

Ericsson, K. A., Prietula, M. J., \& Cokely, E. T. (2007). The making of an expert. Harvard Business Review, 85(7-8), 114-121, 193.

Ericsson, K. A., Whyte, J. I., \& Ward, P. (2007). Expert performance in nursing: reviewing research on expertise in nursing within the framework of the expertperformance approach. Advances in Nursing Science, 30(1), E58-E71.

Ertmer, P. A., Stepich, D. A., Flanagan, S., Kocaman-Karoglu, A., Reiner, C., Reyes, L., Santone, A. L. \& Ushigusa, S. (2009). Impact of guidance on the problem-solving efforts of instructional design novices. Performance Improvement Quarterly, 21(4), 117-132. doi: 10.1002/piq.20041.

Ertmer, P. A., Stepich, D. A., York, C. S., Stickman, A., Wu X. (L.), Zurek, S. \& Goktas, Y. (2008). How instructional design experts use knowledge and experience to solve ill-structured problems. Performance Improvement, 21(1), 17-42.

Fadde, P. J. \& Klein, G. A. (October 2010) Deliberate performance: accelerating expertise in natural settings. Performance Improvement, 49(9), 5-14.

Faure, M., Rosenzweig, J. \& Van Tiem, D. (2010). Appreciative inquiry. In R. Watkins \& D. Leigh (Eds.), Handbook of improving performance in the workplace volume 2: selecting and implementing performance interventions, (pp. 141-166). San Francisco, CA: Pfeiffer. 
Feltovich, P. J., Johnson, P. E., Moller, J. H., \& Swanson, D. B. (1984). LCS: the role and development of medical knowledge in diagnostic expertise. In W. J. Clancey \& E. H. Shortcliffe (Eds.), READINGS in medical artificial intelligence (pp. 275319). Reading, MA: Addison-Wesley.

Field, A. (2009). Discovering statistics using SPSS and sex, drugs, and rock'n' roll (3rd ed.). London: Sage Publications.

Fitzpatrick, J. L., Sanders, J. R. \& Worthen, B. R. (2004). Program evaluation: alternative approaches and practical guidelines (3rd ed.). Boston, MA: Pearson Education, Inc.

Fiske, S. T., Kinder, D. R., \& Larter, W. M. (1983). The novice and the expert: Knowledge-based strategies in political cognition. Journal of Experimental Social Psychology, 19(4), 381-400. doi: 10.1016/0022-1031(83)90029-x

Ford, D. N., \& Sterman, J. D. (1998). Expert knowledge elicitation to improve formal and mental models. System Dynamics Review, 14(4), 309-340. doi: 10.1002/(sici)1099-1727(199824)14:4<309::aid-sdr154>3.0.co;2-5

Foshay, W. R., \& Moller, L. (1992). Advanced the field through research. In H. D. Stolovitch \& E. J. Keeps (Eds.), Handbook of human performance technology (pp. 701 - 714). San Francisco, CA: Jossey-Bass.

Foshay, W. R., Moller, L., Schwen, T. M., Kalman, H. K., \& Haney, D. S. (1999). Research in human performance technology. In H. D. Stolovitch \& E. J. Keeps (Eds.), Handbook of human performance technology (2nd ed.) (pp. 895 - 915). San Francisco, CA: Jossey-Bass. 
Fox, E. J. \& Klein, J. D. (2003). What should instructional designers \& technologists know about human performance technology? Performance Improvement Quarterly, 16(3), 87-98.

Gardiner, M. M., \& Christie, B. (1987). Applying cognitive psychology to user-interface design. Chichester; New York: Wiley.

Gentner, D., \& Stevens, A. L. (1983). Mental models. Hillsdale, NJ: Lawrence Erlbaum Associates, Publishers.

Giberson, T. R. (2010). Performance capabilities and competencies at the undergraduate and graduate levels for performance improvement professionals. Performance Improvement Quarterly, 22(4), 99-120.

Gilbert, T. F. (1978). Human competence: Engineering worthy performance. New York: McGraw Hill.

Gobet, F., \& Charness, N. (2006). Expertise in chess. In R. R. Hoffmann (Ed.), The Cambridge handbook of expertise and expert performance (pp. 523-539). New York: Cambridge University.

Gobet, F., \& Chassy, P. (2008). Towards an alternative to Benner's theory of expert intuition in nursing: A discussion paper. International Journal of Nursing Studies, 45(1), 129-139. doi: 10.1016/j.ijnurstu.2007.01.005.

Goldsmith, T. E., \& Davenport, D. M. (1990). Assessing structural similarity of graphs. In R. W. Schvaneveldt (Ed.), Pathfinder associative networks: studies in knowledge organization (pp. 75-87). Norwood, NJ: Ablex Publishing Corp. 
Goldsmith, T. E., \& Johnson, P. J. (1990). A structural assessment of classroom learning. In R. W. Schvaneveldt (Ed.), Pathfinder associative networks: studies in knowledge organization (pp. 241-254). Norwood, NJ: Ablex Publishing Corp.

Goldsmith, T. E., Johnson, P. J., \& Acton, W. H. (1991). Assessing Structural Knowledge. Journal of Educational Psychology, 83(1), 88-96.

Grabner, R., Stern, E., \& Neubauer, A. (2007). Individual differences in chess expertise: A psychometric investigation. Acta Psychologica, 124(3), 398-420. doi: 10.1016/j.actpsy.2006.07.008

Gray, S. (1990). Using protocol analyses and drawings to study mental model construction during hypertext navigation. International journal of human-computer interaction, 2(4), 359-378. doi: 10.1080/10447319009525990

Guerra-Lopez, I. \& Leigh, H. N. (2009). Are performance improvement professionals measurably improving performance? What PIJ and PIQ have to say about the current use of evaluation and measurement in the field of performance improvement. Performance Improvement Quarterly, 22(2), 97-110.

Guerra, I. J. (2003). Key competencies required of performance improvement professionals. Performance Improvement Quarterly, 16(1), 55-72.

Hardré, P. L., Ge, X. \& Thomas. M. K. (2006). An investigation of development toward instructional design expertise. Performance Improvement Quarterly, 19(4), 63-90.

Haerem, T., \& Rau, D. (2007). The influence of degree of expertise and objective task complexity on perceived task complexity and performance. Journal of Applied Psychology, 92(5), 1320-1331. doi: 10.1037/0021-9010.92.5.1320 
Heracleous, L. (2002). Strategic thinking or strategic planning? Long Range Planning, 31(3), 481-487.

Hill, B. R. (2006). Eliciting, analyzing, and comparing mental models of complex audio recording systems between professional and novice recording engineers (D.Ed. dissertation, Pennsylvania State University, Pennsylvania, United States). Retrieved from November 22, 2010, Dissertations \& Theses: Full Text (Publication No. AAT 3229407)

Hill, N. M., \& Schneider, W. (2006). Brain changes in the development of expertise: neuroanatomical and neurophysiological evidence about skill-based adaptations. In R. R. Hoffmann (Ed.), The Cambridge handbook of expertise and expert performance (pp. 653-682). New York: Cambridge University.

Hmelo-Silver, C. E., Marathe, S., \& Liu, L. (2007). Fish swim, rocks sit, and lungs breathe: Expert-novice understanding of complex systems. The Journal of the Learning Sciences, 16(3), 307.

Hmelo-Silver, C. E., \& Pfeffer, M. G. (2004). Comparing expert and novice understanding of a complex system from the perspective of structures, behaviors, and functions. Cognitive Science, 28(1), 127-138. doi: 10.1016/s03640213(03)00065-x

Hodgkinson, G. P. (2002). Comparing managers' mental models of competition: why self-report measures of belief similarity won't do. Organization Studies, 23(1), 6372. doi: $10.1177 / 0170840602231003$ 
Hoffman, R. R. (1998). How can expertise be defined?: Implications of research from cognitive psychology. In R. Williams, W. Faulkner \& J. Fleck (Eds.), Exploring expertise (pp. 81-100). Cambridge, MA: MIT Press.

Hoffman, R. R., Feltovich, P. J., \& Ford, K. M. (1997). Cognitive conceptions of expertise. In P. J. Feltovich, K. M. Ford \& R. R. Hoffman (Eds.), Expertise in context. Human and machine (pp. 149-162). Menlo Park, CA: AAAI Press/The MIT Press.

Honeck, R. P., \& Temple, J. G. (1992). Metaphor, expertise, and a PEST: Comments on the contributions to this special issue. Metaphor and symbol, 7(3), 237-252. doi: 10.1207/s15327868ms0703\&4_8

Horn, J., \& Masunaga, H. (2006). A merging theory of expertise and intelligence. In R. R. Hoffmann (Ed.), The Cambridge handbook of expertise and expert performance (pp. 587-612). New York: Cambridge University.

Hsu, Y. (2006). The effects of metaphors on novice and expert learners' performance and mental-model development. Interacting with Computers, 18(4), 770-792. doi: 10.1016/j.intcom.2005.10.008

Huglin, L., Johnsen, L., \& Marker, A. (2007). Research priorities in performance technology a delphi study. Performance Improvement Quarterly, 20(1), 79-95.

Ifenthaler, D., Masduki, I., \& Seel, N. M. (2009). The mystery of cognitive structure and how we can detect it: tracking the development of cognitive structures over time. Instructional Science. doi: 10.1007/s11251-009-9097-6

Interlink. (n.d.). KNOT. Retrieved November 2, 2010, from http://interlinkinc.net/KNOT.html 
International Society for Performance Improvement - ISPI (2012). ISPI Performance Standards. Retrieved on Dec 08, 2011 from http://www.ispi.org/uploadedFiles/ISPI_Site/About_ISPI/About/Standards.pdf Jacoby, S., \& Gonzales, P. (1991). The constitution of expert-novice in scientific discourse. Issues in applied linguistics, 2(2), 149.

Januzewski, A., \& Molenda, M. (Eds.). (2007). Educational technology: a definition with commentary. Florence, KY: Routledge.

Jih, H., \& Reeves, T. (1992). Mental models: A research focus for interactive learning systems. Educational Technology Research and Development, 40(3), 39-53. doi: 10.1007/bf02296841

Johnsen, L. V., Huglin, L. M., \& Marker, A. (2008). Primary sources of cognate research in human performance technology. Performance Improvement Quarterly, 21(1), 7-16. doi: 10.1002/piq.20012

Johnson, B. \& Christensen, L. (2008). Educational research: quantitative, qualitative, and mixed approaches (3rd ed.) Thousand Oaks, CA: Sage Publications Inc.

Johnson-Laird, P. N. (1980). Mental models in cognitive science. Cognitive Science, 4(1), 71-115. doi: 10.1207/s15516709cog0401_4

Johnson-Laird, P. N. (1983). Mental models: towards a cognitive science of language, inference, and consciousness. Cambridge, MA: Harvard University Press. Johnson, P. E., Durán, A. S., Hassebrock, F., Moller, J., Prietula, M., Feltovich, P. J., \& Swanson, D. B. (1981). Expertise and error in diagnostic reasoning. Cognitive Science, 5(3), 235-283. doi: Doi: 10.1016/s0364-0213(81)80014-6 
Jonassen, D. H. (1995). Operationalizing mental models: strategies for assessing mental models to support meaningful learning and design-supportive learning environments. Paper presented at the first international conference on Computer support for collaborative learning, Indiana Univ., Bloomington, Indiana, United States.

Kahler, S. E. (2003). A comparison of knowledge acquisition methods for the elicitation of procedural mental models. (Ph.D. dissertation, North Carolina State University, Raleigh, North Carolina, United States.) Retrieved from December 01, 2010, Dissertations \& Theses: Full Text (Publication No. AAT 3071491)

Kaufman, R., \& English, F. W. (1979). Needs assessment: Concept and application. Englewood Cliffs, NJ: Educational Technology Publications.

Kaufman, R., Oakley-Browne, H., Watkins, R. \& Leigh, D. (2003). Strategic planning for success: aligning people, performance, and payoffs. San Francisco, CA: JosseyBass/Pfeiffer. Kellogg, R. T. (2006). Professional writing expertise. In R. R. Hoffmann (Ed.), The Cambridge handbook of expertise and expert performance (pp. 389-402). New York: Cambridge University.

Kieras, D. E., \& Bovair, S. (1984). The role of a mental model in learning to operate a device. Cognitive Science, 8(3), 255-273. doi: 10.1207/s15516709cog0803_3

Kirschner, P., Carr, C., van Merriënboer, J., \& Sloep, P. (2002). How expert designers design. Performance Improvement Quarterly, 15(4), 86-104. doi: 10.1111/j.19378327.2002.tb00267.x 
Kitsantas, A., \& Zimmerman, B. J. (2002). Comparing self-regulatory processes among novice, non-expert, and expert volleyball players: a microanalytic study. Journal of Applied Sport Psychology, 14, 91-105.

Klein, J. D. (2002). Empirical research on performance improvement. Performance Improvement Quarterly, 15(1), 99-110.

Kohl, P. B., \& Finkelstein, N. D. (2008). Patterns of multiple representation use by experts and novices during physics problem solving. Physical Review Special Topics - Physics Education Research, 4(1), 010111.

Köpke, B., \& Nespoulous, J.-L. (2006). Working memory performance in expert and novice interpreters. Interpreting, 8, 1-23. doi: 10.1075/intp.8.1.02kop

Krampe, R. T., \& Charness, N. (2006). Aging and expertise. In R. R. Hoffmann (Ed.), The Cambridge handbook of expertise and expert performance (pp. 723-742). New York: Cambridge University.

Lau, W. W. F., \& Yuen, A. H. K. (2009). Predictive validity of measures of the pathfinder scaling algorithm on programming performance: alternative assessment strategy for programming education. Journal Of Educational Computing Research, 41(2), 227-250. doi: 10.2190/EC.41.2.e

Lau, W. W. F., \& Yuen, A. H. K. (2010). Promoting conceptual change of learning sorting algorithm through the diagnosis of mental models: The effects of gender and learning styles. Computers \& Education, 54(1), 275-288. doi: 10.1016/j.compedu.2009.08.014

Lauer, M. (2008). Validating the ISPI Standards and Principles for the Certified Performance Technologist Credential. (Ph.D. dissertation, Indiana University, 
United States - Indiana). Retrieved December 15, 2011, from Dissertations \& Theses: Full Text. (Publication No. AAT 3331327).

Lave, J., \& Wenger, E. (1991). Situated learning: legitimate peripheral participation. Cambridge: Cambridge University Press.

Le Maistre, C. (1998). What is an expert instructional designer? Evidence of expert performance during formative evaluation. Educational Technology Research and Development, 46(3), 21-36. doi: 10.1007/bf02299759

Lee, L. (2008). Focus-on-form through collaborative scaffolding in expert-to-novice online interaction. Language Learning \& Technology, 12(3), 53.

Leigh, H. N. \& Tracey, M. W. (2010). A review and new framework for instructional design practice variation research. Performance Improvement Quarterly, 23(2), 33-46.

Lister, R., Simon, B., Thompson, E., Whalley, J. L., \& Prasad, C. (2006). Not seeing the forest for the trees: novice programmers and the SOLO taxonomy. SIGCSE Bull., 38(3), 118-122. doi: 10.1145/1140123.1140157

Marker, A., Huglin, L. \& Johnsen, L. (2006). Empirical research on performance improvement: An update. Performance Improvement Quarterly, 19(4), 7-22.

Masunaga, H., \& Horn, J. (2000). Characterizing mature human intelligence: Expertise development. Learning and Individual Differences, 12(1), 5-33. doi: $10.1016 / \mathrm{s} 1041-6080(00) 00038-8$

Mathieu, J. E., Heffner, T. S., Goodwin, G. F., Salas, E., \& Cannon-Bowers, J. A. (2000). The influence of shared mental models on team process and performance. Journal of Applied Psychology, 85(2), 273-283. 
McGaghie, W. C., McCrimmon, D. R., Mitchell, G., Thompson, J. A., \& Ravitch, M. M. (2000). Quantitative concept mapping in pulmonary physiology: comparison of student and faculty knowledge structures. Advance Physiological Education, 23(1), 72-81.

McKeithen, K. B., Reitman, J. S., Rueter, H. H., \& Hirtle, S. C. (1981). Knowledge organization and skill differences in computer programmers. Cognitive Psychology, 13(3), 307-325. doi: 10.1016/0010-0285(81)90012-8.

Means, M. L., \& Voss, J. F. (1985). Star Wars: A developmental study of expert and novice knowledge structures. Journal of Memory and Language, 24(6), 746-757. doi: 10.1016/0749-596x(85)90057-9

Mieg, H. A. (2006). Social and sociological factors in the development of expertise. In R. R. Hoffmann (Ed.), The Cambridge handbook of expertise and expert performance (pp. 743-760). New York: Cambridge University.

Mitchell, R., \& Unsworth, C. A. (2005). Clinical reasoning during community health home visits: expert and novice differences. The British Journal of Occupational Therapy, 68, 215-223.

Molenda, M. \& Pershing, J. A. (2004). An integrative approach to performance improvement and instructional systems design. TechTrends, 48(2), 26-32.

Moseley, J. L. \& Dessinger, J. C. (2007). Training older workers and learners. San Francisco, CA: Pfeiffer.

Nation, R., \& Mclaughlin, B. (1986). Novices and experts: An information processing approach to the "good language learner" problem. Applied Psycholinguistics, 7, 41-55. doi: 10.1017/S0142716400007177 
Nelson, W. (1989). Artificial intelligence knowledge acquisition techniques for instructional development. Educational Technology Research and Development, 37(3), 81-94. doi: 10.1007/bf02299060

Norman, D. A. (1983). Some observations on mental models. In D. Gentner \& A. L. Stevens (Eds.), Mental Models (pp. 7-14). Hillsdale, NJ: Lawrence Erlbaum Associates, Publishers.

Norman, G., Eva, K., Brooks, L., \& Hamstra, S. (2006). Expertise in medicine and surgery. In R. R. Hoffmann (Ed.), The Cambridge handbook of expertise and expert performance (pp. 339-354). New York: Cambridge University.

Osborne, J. D., Stubbart, C. I., \& Ramaprasad, A. (2001). Strategic groups and competitive enactment: a study of dynamic relationships between mental models and performance. Strategic Management Journal, 22(5), 435-454.

Parry, S. B. (1996). The quest for competencies. Training, 33(7), 48-54.

Payne, S. J. (1991). A descriptive study of mental models (Vol. 10). Colchester, ROYAUME-UNI: Taylor \& Francis.

Perez, R. S., \& Emery, C. D. (1995). Designer thinking: how novices and experts think about instructional design. Performance Improvement Quarterly, 8(3), 80-95. doi: 10.1111/j.1937-8327.1995.tb00688.x

Perez, R. S., Fleming Johnson, J., \& Emery, C. D. (1995). Instructional design expertise: A cognitive model of design. Instructional Science, 23(5), 321-349. doi: $10.1007 / \mathrm{bf00896877}$ 
Pershing, J. A. (2006). Human performance technology fundamentals. In J. A. Pershing, (Ed.) Handbook of human performance technology: principles, practices, potential (3rd ed.), (pp 5-34). San Francisco, CA: Pfeiffer.

Pershing J. A., Lee J., Cheng, J. (Feb 2008b). Current status, future trends, and issues in human performance technology, part 2: Models, influential disciplines, and research and development. Performance Improvement, 47(2), 7-15.

Pershing J. A., Lee J., Cheng, J. (Jan 2008a). Current status, future trends, and issues in human performance technology, part 1: Models, influential disciplines, and research and development. Performance Improvement, 47(1), 9-17.

Phillips, J. K., Klein, G., \& Sieck, W. R. (2008). Expertise in judgment and decision making: a case for training intuitive decision skills. In D.J. Koehler, N. Harvey (Eds.), Blackwell handbook of judgment and decision making, (pp 295-315). Oxford, UK: Blackwell Publishing Ltd.

Phillips, P. P. \& Phillips, J. J. (2007). The value of learning: how organizations capture value and roi and translate them into support, improvement, and funds. San Francisco, CA: Pfeiffer.

Posner, M. I. (1988). Introduction: what is it to be an expert? In M. T. H. Chi, R. Glaser \& M. J. Farr (Eds.), The nature of expertise, (pp. xxix - xxxvi). Hillsdale, N.J.: Lawrence Erlbaum Associates, Inc., Publishers.

Randel, J. M., Pugh, H. L., \& Reed, S. K. (1996). Differences in expert and novice situation awareness in naturalistic decision making. International Journal of Human-Computer Studies, 45(5), 579-597. doi: 10.1006/ijhc.1996.0068 
Rawson, K. A., \& Van Overschelde, J. P. (2008). How does knowledge promote memory? The distinctiveness theory of skilled memory. Journal of Memory and Language, 58(3), 646-668. doi: 10.1016/j.jml.2007.08.004

Rentsch, J. R., Mot, I., \& Abbe, A. (2009). Identifying the Core Content and Structure of a Schema for Cultural Understanding (pp. 1-31). Arlington, VA: U.S. Army Research Institute for the Behavioral and Social Sciences.

Rose, J., Rose, A., \& McKay, B. (2007). Measurement of knowledge structures acquired through instruction, experience, and decision aid use. International Journal of Accounting Information Systems, 8(2), 117-137. doi: 0.1016/j.accinf.2007.04.002

Ross, K. G., Battaglia, D., Phillips, J., Domeshek, E. A., \& Lussier, J. W. (2003). Mental models underlying tactical thinking skills. Paper presented at the The Interservice/Industry Training, Simulation \& Education Conference (I/ITSEC).

Ross, K. G., Shafer, J. L., \& Klein, G. (2006). Professional judgments and "naturalistic decision making". In R. R. Hoffmann (Ed.), The Cambridge handbook of expertise and expert performance (pp. 403-420). New York: Cambridge University.

Rothwell, W. (1996). Beyond training and development: State-of-the-art strategies for enhancing human performance. New York: AMACOM.

Rouse, W. B., \& Morris, N. M. (1985). On looking into the black box: prospects and limits in the search for mental models (pp. 1-61). Atlanta, GA: Center for ManMachine Systems Research, School of Industrial \& System Engineering, Georgia Institute of Technology. 
Rouse, W. B., \& Morris, N. M. (1986). On looking into the black box: prospects and limits in the search for mental models. Psychological Bulletin, 100(3), 349-363. doi: 10.1037/0033-2909.100.3.349

Rowe, A. L., \& Cooke, N. J. (1995). Measuring mental models: Choosing the right tools for the job. Human Resource Development Quarterly, 6(3), 243-255. doi: 10.1002/hrdq.3920060303

Rowe, L. J., Schvaneveldt, R. W., \& Bennett, W. J. (2007). Measuring pilot knowledge in training: the pathfinder network scaling technique. Paper presented at the The Interservice/Industry Training, Simulation \& Education Conference (I/ITSEC).

Rowland, G. (1992). What do instructional designers actually do? An initial investigation of expert practice. Performance Improvement Quarterly, 5(2), 65-86. doi: 10.1111/j.1937-8327.1992.tb00546.x

Rymarchyk, G. K. (1996). Validity. Research Methods Tutorials. Retrieved October 5, 2010, from http://www.socialresearchmethods.net/tutorial/Rymarchk/rymar2.htm

Rummler, G. A. (2007). Serious performance consulting: according to Rummler. San Francisco, CA: Pfeiffer.

Salas, E., Rosen, M. A., Burke, C. S., Goodwin, G. F., \& Fiore, S. M. (2006). The making of a dream team: when expert teams do best. In R. R. Hoffmann (Ed.), The Cambridge handbook of expertise and expert performance (pp. 439-453). New York: Cambridge University.

Salas, E., Rosen, M. A., \& DiazGranados, D. (2010). Expertise-based intuition and decision making in organizations. Journal of Management, 36(4), 941-973. doi: $10.1177 / 0149206309350084$ 
Schlomske, N., \& Pirnay-Dummer, P. (2009). Model based assessment of learning dependent change within a two semester class. Educational Technology Research and Development, 57(6), 753-765. doi: 10.1007/s11423-009-9125-x

Schoenfeld, A. H., \& Herrmann, D. J. (1982). Problem perception and knowledge structure in expert and novice mathematical problem solvers. Journal of Experimental Psychology: Learning, Memory, and Cognition, 8(5), 484-494. doi: $10.1037 / 0278-7393.8 .5 .484$

Schraw, G., \& Moshman, D. (1995). Metacognitive theories. Educational Psychology Review, 7(4), 351-371. doi: 10.1007/bf02212307

Schuelke, M. J., Day, E. A., McEntire, L. E., Boatman, P. R., Boatman, J. E., Kowollik, V., \& Wang, X. (2009). Relating indices of knowledge structure coherence and accuracy to skill-based performance: Is there utility in using a combination of indices? Journal of Applied Psychology, 94(4), 1076-1085. doi: 0.1037/a0015113

Schvaneveldt, R. W. (1990). Proximities, networks, and schemata. In R. W.

Schvaneveldt (Ed.), Pathfinder associative networks: studies in knowledge organization (pp. 135-148). Norwood, NJ: Ablex Publishing Corp.

Schvaneveldt, R. W. (n.d.). Pathfinder 6.3 documentation. Retrieved October 11, 2010, from http://interlinkinc.net/Pathfinder.doc

Schvaneveldt, R. W., Durso, F. T., Goldsmith, T. E., Breen, T. J., Cooke, N. M., Tucker, R. G., \& De Maio, J. C. (1985). Measuring the structure of expertise. International Journal of Man-Machine Studies, 23(6), 699-728. doi: 10.1016/s00207373(85)80064-x 
Self-Report Method. (2008). International encyclopedia of the social sciences. Retrieved October 12, 2010, from ttp://www.encyclopedia.com/doc/1G2-3045302394.html Serfaty, D., MacMillan, J., Entin, E. E., \& Entin, E. B. (1997). The decision-making expertise of battle commanders. In C. E. Zsambok \& G. Klein (Eds.), Naturalistic decision making (pp. 233-246). Mahwah, NJ: Lawrence Erlbaum Associates.

Schaffer, S. P. \& Keller, J. (2003) Measuring results of performance improvement interventions. Performance Improvement Quarterly, 16(1), 73-92.

Sheehan, K. B. (2001). E-mail survey response rates: A Review. Journal of ComputerMediated Communication, 6(2), 0-0. doi: 10.1111/j.1083-6101.2001.tb00117.x

Silber, K. H. \& Foshay, W. R. (2010). Handbook of improving performance in workplace volume one: instructional design and training delivery. San Fransicso, CA: Pfeiffer.

Smith-Jentsch, K. A., Campbell, G. E., Milanovich, D. M., \& Reynolds, A. M. (2001). Measuring teamwork mental models to support training needs assessment, development, and evaluation: two empirical studies. Journal Of Organizational Behavior, 22(2), 179-194. doi: 10.1002/job.88

Stefanidis, D., Korndorffer Jr, J. R., Markley, S., Sierra, R., Heniford, B. T., \& Scott, D. J. (2007). Closing the gap in operative performance between novices and experts: does harder mean better for laparoscopic simulator training? Journal of the American College of Surgeons, 205(2), 307-313. doi:

10.1016/j.jamcollsurg.2007.02.080 
Sternberg, R. J. (1997). Cognitive conceptions of expertise. In P. J. Feltovich, K. M. Ford \& R. R. Ho!man (Eds.), Expertise in context. Human and machine (pp. 149162). Menlo Park, CA: AAAI Press/The MIT Press.

Stolovitch, H. D. (2000). Human performance technology: Research and theory to practice. Performance Improvement, 39(4), 7-16.

Sugrue, B., \& Stolovitch, H. D. (2000). Report of 1999 ISPI symposium appropriate inquiry in human performance technology. Performance Improvement, 39(1), 3336.

Suter, W. N. (2006). Introduction to educational research: a critical thinking approach. Thousand Oaks, CA: Sage Publications Inc.

Swanson, R. A. (1988). Research and development (and other life and death matters). Performance Improvement Quarterly, 1(1), 69-82.

Tabachnick, B. G., \& Fidell, L. S. (2007). Using multivariate statistics (5th ed.). Boston, MA: Pearson Education. Inc.

Tan, H. T. (1997). Tacit managerial versus technical knowledge as determinants of audit expertise in the field. Journal of Accounting Research, 35(1), 97.

Taricani, E., \& Clariana, R. (2006). A technique for automatically scoring open-ended concept maps. Educational Technology Research and Development, 54(1), 6582. doi: 10.1007/s11423-006-6497-z.

Thompson, L. F., Surface, E. A., Martin, D. L., \& Sanders, M. G. (2003). From paper to pixels: Moving personnel surveys to the Web. Personnel Psychology, 56 (1), 197-227. 
Trochim, M. K. (2006). Measurement validity types. Research methods knowledgebase. Retrieved on Dec 15, 2011 from http://www.socialresearchmethods.net/kb/measval.php.

Trumpower, D. L., Sharara, H., \& Goldsmith, T. E. (2010). Specificity of structural assessment of knowledge. Journal of Technology, Learning, and Assessment, 8(5). Retrieved from http://escholarship.bc.edu/jtla/vol8/5/

Tversky, B. (1993). Cognitive maps, cognitive collages, and spatial mental models. In A. Frank \& I. Campari (Eds.), Spatial Information Theory A Theoretical Basis for GIS Vol. 716, (pp. 14-24): Springer Berlin / Heidelberg.

Tynjälä, P. (1999). Towards expert knowledge? A comparison between a constructivist and a traditional learning environment in the university. International Journal of Educational Research, 31(5), 357-442. doi: 10.1016/s0883-0355(99)00012-9

Vadivelu, R. N. \& Klein, J. D. (2008). Cross-cultural analysis of HPT: An empirical investigation of HPT competencies in the workplace in the United States and South Asia. Performance Improvement Quarterly, 20(3-4), 147-165.

Van Tiem, D. M. (2004) Interventions (solutions) usage and expertise in performance technology practice: An empirical investigation. Performance Improvement Quarterly, 17(3), 23-44.

Van Tiem, D. M., Moseley, J. L. \& Dessinger, J. C. (2012). Fundamentals of performance improvement: A guide to optimizing results through people, processes and organizations (3rd ed.). San Francisco: Pfeiffer/Wiley.

Villachica, S. W., Lohr, L. L., Summers, L., Lowell, N., Roberts, S., Javeri, M., Conn, C. (2001, November 8-12). A cognitive map of human performance technology: a 
study of domain expertise. Paper presented at the 24th Annual Proceedings of Selected Research and Development and Practice Papers Presented at the National Convention of the Association of Educational Communications and Technology Atlanta, GA.

Villachica, S. W. (1999). An investigation of the stability of Pathfinder-related measures. (Ph.D. dissertation, University of Northern Colorado, United States -Colorado.) Retrieved December 16, 2011, from Dissertations \& Theses: Full Text.(Publication No. AAT 9955552).

Voss, J. F., \& Wiley, J. (2006). Expertise in history. In R. R. Hoffmann (Ed.), The Cambridge handbook of expertise and expert performance (pp. 569-584). New York: Cambridge University.

Wagner, R. K. (1987). Tacit knowledge in everyday intelligent behavior. Journal of Personality and Social Psychology, 52(6), 1236-1247.

Wallen, N. E., \& Fraenkel, J. R. (2001). Educational research : A guide to the process. Mahwah, NJ: Lawrence Erlbaum Associates, Inc., Publishers.

Waters, A. J., \& Gobet, F. (2008). Mental imagery and chunks: Empirical and computational findings. Memory \& Cognition, 36(3), 505-517. doi: 10.3758/mc.36.3.505

Weisberg, R. W. (2006). Modes of expertise in creative thinking: evidence from case studies. In R. R. Hoffmann (Ed.), The Cambridge handbook of expertise and expert performance (pp. 761-787). New York: Cambridge University. 
Winn, W. (2004). Cognitive perspectives in psychology. In D. H. Jonassen (Ed.), Handbook of research on educational communications and technology (2nd ed.) (pp. 79 - 112). Mahwah, NJ: Lawrence Erlbaum Associates, Inc.

Witucki, A. P. (2006). Factors affecting knowledge and usage of specific performance improvement interventions by novice and expert performance technologists (Ph.D. dissertation, Wayne State University, Detroit, Michigan, United States.) Retrieved from October 12, 2010, Dissertations \& Theses @ Wayne State University (Publication No. AAT 3210994)

Wright, K. B. (2005). Researching internet-based populations: advantages and disadvantages of online survey research, online questionnaire authoring software packages, and web survey services. Journal of Computer-Mediated Communication, 10(3), 00-00. doi: 10.1111/j.1083-6101.2005.tb00259.x

Zahodnic, R. (2009). The use of a think-aloud protocol as an instructional strategy to improve novice decision making (Ph.D. dissertation, Wayne State University, United States - Michigan). Retrieved from October 27, 2010, Dissertations \& Theses @ Wayne State University. (Publication No. AAT 3387447)

Zimmerman, B. J. (2006). Development and adaptation of expertise: the role of selfregulatory processes and beliefs. In R. R. Hoffmann (Ed.), The Cambridge handbook of expertise and expert performance (pp. 705-722). New York: Cambridge University. 


\section{ABSTRACT}

\section{THE MENTAL MODEL COMPARISON OF EXPERT AND NOVICE PERFORMANCE IMPROVEMENT PRACTITIONERS}

by

\section{SACIP TOKER}

\section{May 2012}

Advisor: Dr. James L. Moseley

Major: Instructional Technology

Degree: Doctor of Philosophy

The primary purpose of this study was to reveal performance improvement practitioner expert and novice mental models and identify differences and similarities between these models. The secondary purpose was to analyze the potential relationships of the professional profile characteristics of performance improvement practitioners with their mental model of expertise derived from Pathfinder scaling algorithm. The study was stemmed from one of the critical research trends in the field of Human Performance Technology (HPT).

There are two phases of the study. In the first round of the first phase, experts, who were selected based on several criteria, were contacted to identify the most critical concepts related the HPT. The Online Ranking Questionnaire was utilized. 23 experts were responded, and 11 of 30 concepts were selected. In the second round of the first phase, the experts who responded to the first round were contacted again to share their professional profile characteristics and ratings about the concept-pairs generated from the 11 concepts. These ratings provided the proximity data necessary to generate the 
common mental models of expert (the expert referent model) in the KNOT using the Pathfinder algorithm. The Professional Profile Characteristics and The Concept-Pairs Comparison online questionnaires were used. 16 experts responded in this round. In the second phase of the study, practitioners in the field were invited to participate in the study via International Society for Performance Improvement (ISPI) newsletters, The American Society for Training and Development (ASTD) discussion forums, and Association for Educational Communications and Technologies (AECT) mailing list. Moreover, professional social networking sites, e.g., Linked-In, and the researcher personal contact list were used as well to increase return-rate. Practitioners were asked to complete the same online questionnaires completed by the experts in the second round of the first phase. 335 practitioners started the questionnaires; 272 completed the Professional Profile Characteristics questionnaire; 242 completed both the Professional Profile Characteristics and the Concept-Pairs Comparison questionnaires. 33 practitioners of 242 were identified as novices who were selected based on the criteria used to select experts. In contrast to the experts, the novices were chosen as those who do not meet all of the criteria. The proximity data of those 33 novices were used to create the common mental model of novices.

The common mental model of experts demonstrated more coherent and hierarchical structure. However, the common mental model of novices was in more linear structure. The models were also compared, and the experts' model was different from the novices' model. The expert model had deep structure of practical knowledge; whereas, the novice model contained step-by-step and textbook style structure. The professional profile characteristics of the practitioners and the experts were also 
presented. Several relationships found between the professional profile characteristics and the mental model of expertise, which was generated from three Pathfinder measures: relatedness, coherence, and similarity. The mental model of expertise was positively associated with the number of organizations worked, the number of completed projects, the diversity of project types, the number of the HPT related courses taught; whereas, it was negatively associated with the total years spent to earn degrees.

There were several implications of the current study. The first is either informal or formal approaches for the development of expertise. This study may enlighten the mentoring novices while progressing to expertise in the field. Colleges, universities and other types of institutions providing education or training for performance improvement practitioners may take advantage of the results of this study by improving their course or curriculum designs with additional experiences. Moreover, the professional organizations, such as ISPI and ASTD, may be informed with this study for their certification and designation programs. They may include new rationale and criteria for assessment and evaluation processes. This study also may provide additional information from the expertise perspective to the efforts related to the development of competencies in the performance improvement field.

Finally, future studies were recommended. The first recommendation was the replication of the current study with different sample characteristics and sizes. The future studies regarding expertise in HPT may consider different using different research design and knowledge elicitation techniques. Since the current study utilized stand-alone concepts, the studies examining groups of stand-alone concepts with 
common characteristics may provide more meaningful and overarching interpretations. There were numerous either demographic, e.g., age, or professional, e.g., years of experience, deliberate practice, and so forth, factors influencing in expertise in either general or more specific to performance improvement field. These factors needed to be analyzed to reveal the relationships with the progression to expertise. 


\title{
AUTOBIOGRAPHICAL STATEMENT
}

\author{
Sacip Toker
}

Education: Master of Science in Curriculum and Instruction

Middle East Technical University, Ankara, TURKEY, 2004

Bachelor of Science in Computer Education and Instructional Technology

Middle East Technical University, Ankara, TURKEY, 2001

Employment: Research Analyst

Capital Analytics, (Durham, NC)

June, 2011 - Dec, 2011

Graduate Research Assistant

Wayne State University, College of Education, Administrative and Organizational Studies Division, Instructional Technology Program (Detroit, MI)

August, 2010 - May, 2011

Interactive and Performance Technologist

William Beaumont Hospitals, Corporate Human Resources Information Systems (Royal Oak, MI) 2009 - 2010

Awards: $\quad$ First Place at 3rd Annual University Case Study Competition of International Society for Performance Improvement, Orlando, FL, 2011

Publications: Chapters in Books

Toker, S. \& Moseley, J. L (2012) Cultural Readiness Scale for Web 2.0 (CuReS for Web 2.0) In Biech, E. (ed), The 2012 Pfeiffer Annual: Consulting, (pp. 151-168). San Francisco, CA: Pfeiffer

Toker, S. \& Moseley, J. L. (2011). Competitive intelligence scale for organizations (CISO). In Biech, E. (ed), The 2011 Pfeiffer Annual: Consulting, (pp. 149-160). San Francisco, CA: Pfeiffer.

Toker, S., Moseley, J. L. \& Chow, A. (2010). Scale of intellectual capital for organizations (SICO), In Biech, E. (ed), The 2010 Pfeiffer Annual: Consulting, (pp. 149-166). San Francisco, CA: Pfeiffer.

Moseley, J. L., Toker, S. \& Chow, A. (2009). Mind aptitude scale for organizations (MASO). In Biech, E. (ed), The 2009 Pfeiffer Annual: Consulting, (pp. 137-152). San Francisco, CA: Pfeiffer.

\section{Refereed Journal Articles}

Zhang, K. \& Toker, S. (2012). Stimulating critical thinking in a virtual learning community with instructor moderation and peer review. Knowledge Management \& E-Learning: An International Journal, 3(4), 534-547.

Guerra-Lopez. I \& Toker, S. (2012). An application of the impact evaluation process for designing a performance measurement and evaluation framework in $\mathrm{K}-12$ environments. Evaluation and Program Planning, 35, 222-235

Siko, J. P., Barbour, M. B. \& Toker, S. (2011) Beyond Jeopardy and Lectures: Using Microsoft PowerPoint as a Game Design Tool to Teach Science. Journal of Computers in Mathematics and Science Teaching (JCMST), 30(3), 303-320.

Barbour, M., Kinsella, J., Wicks, M.\& Toker, S. (2009). Continuing change in a virtual world: Training and recruiting instructors. Journal of Technology and Teacher Education, 17(4), 203-224.

\section{Non-refereed Journal Articles}

Moseley, J. L., Toker, S., Fulgham, S. M., Shaughnessy, M. F. (2009). Q \& A with Ed Tech Leaders: Interview with Rita C. Richey. Educational Technology, 49(1), 59-61.

Toker, S., Moseley, J. L. \& Chow, A. T. (2008). Is there a wiki in your future? Educational, instructional design and general use. Educational Technology, 48(5), 22-27. 